101 Prepared in copoperation wh the U.S. Geological Survey Water Availability and Use Science Program

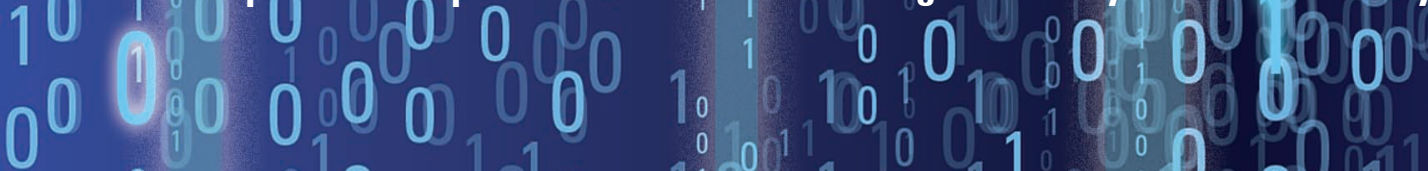

\title{
Documentation for the
}

cochapter 55 of

Section $A$, Groundwater

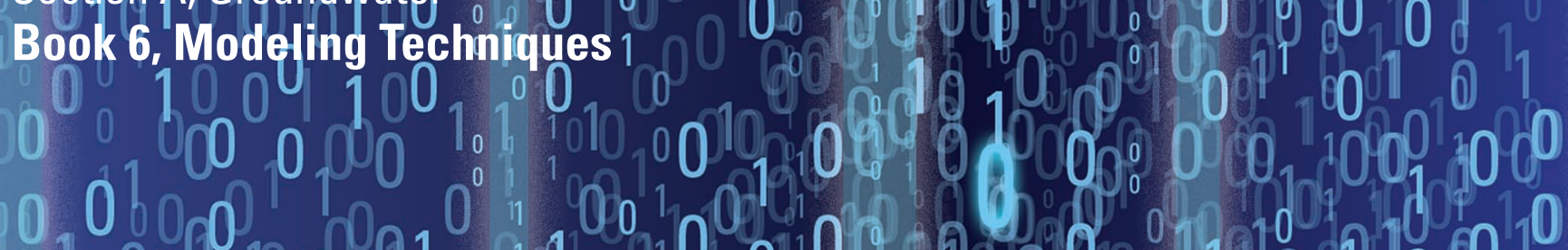

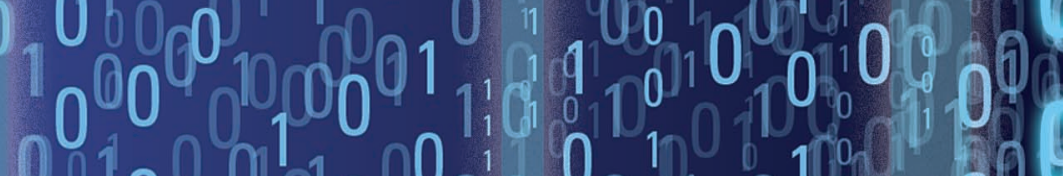

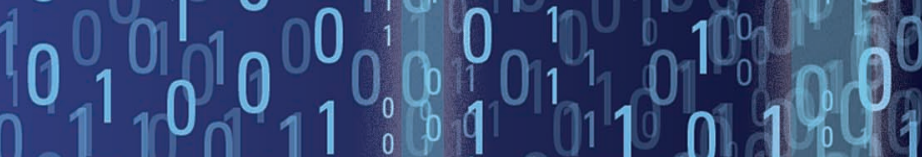

O.

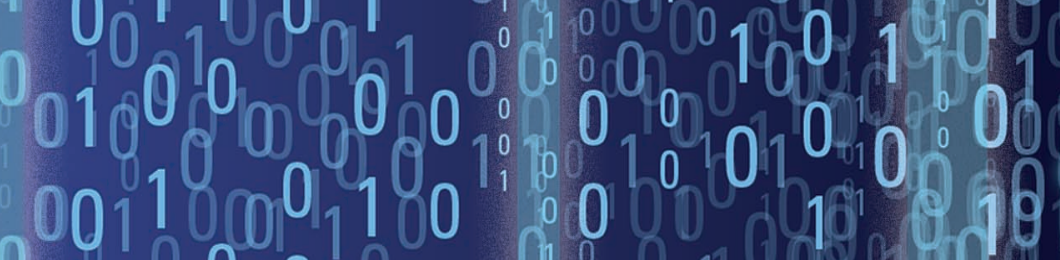

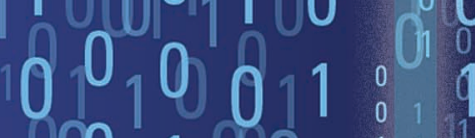

$0.01001 \%$

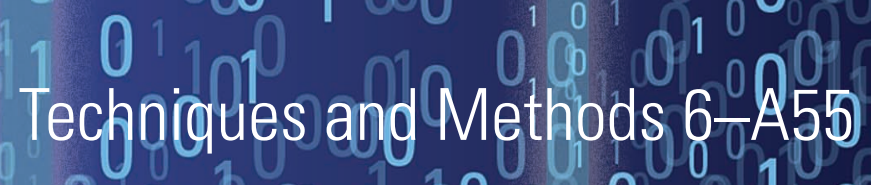<smiles>[Si]1=[Si]=[Si]1</smiles>

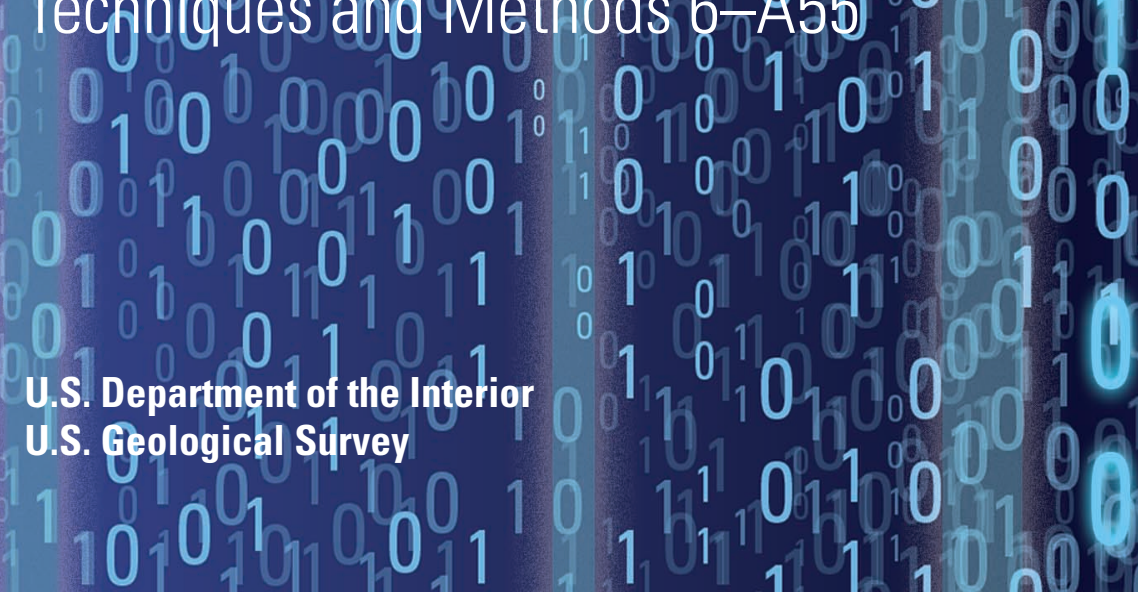

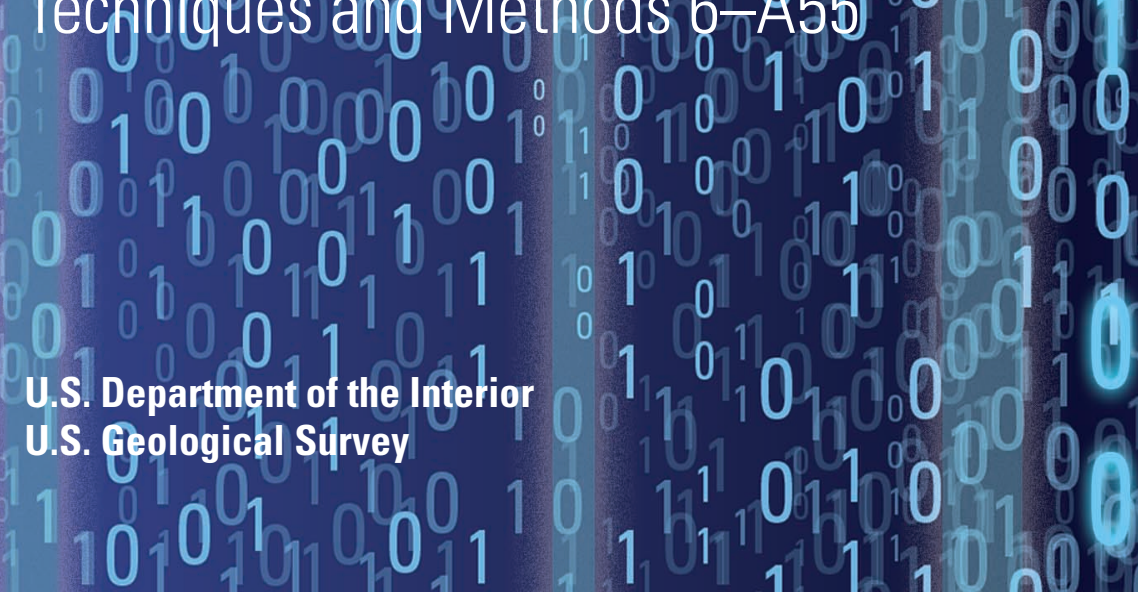

\section{00000100}

1000

0
0
0
1

110

Do 1 1 1 
Cover. Binary computer code illustration. 


\section{Documentation for the MODFLOW 6 Groundwater Flow Model}

By Christian D. Langevin, Joseph D. Hughes, Edward R. Banta, Richard G. Niswonger, Sorab Panday, and Alden M. Provost

Chapter 55 of

Section A, Groundwater

Book 6, Modeling Techniques

Prepared in cooperation with the U.S. Geological Survey Water Availability and Use Science Program

Techniques and Methods 6-A55 


\title{
U.S. Department of the Interior \\ RYAN K. ZINKE, Secretary
}

\author{
U.S. Geological Survey \\ William H. Werkheiser, Acting Director
}

\author{
U.S. Geological Survey, Reston, Virginia: 2017
}

For more information on the USGS - the Federal source for science about the Earth, its natural and living resources, natural hazards, and the environment-visit https://www.usgs.gov or call 1-888-ASK-USGS

For an overview of USGS information products, including maps, imagery, and publications, visit https://store.usgs.gov/.

Any use of trade, product, or firm names in this publication is for descriptive purposes only and does not imply endorsement by the U.S. Government.

Although this information product, for the most part, is in the public domain, it also may contain copyrighted materials as noted in the text. Permission to reproduce copyrighted items must be secured from the copyright owner.

Suggested citation:

Langevin, C.D., Hughes, J.D., Banta, E.R., Niswonger, R.G., Panday, Sorab, and Provost, A.M., 2017, Documentation for the MODFLOW 6 Groundwater Flow Model: U.S. Geological Survey Techniques and Methods, book 6, chap. A55, 197 p., https://doi.org/10.3133/tm6A55.

ISSN 2328-7055 (online) 


\section{Preface}

The report describes the Groundwater Flow Model for the U.S. Geological Survey (USGS) modular hydrologic simulation program called MODFLOW 6. The program can be be downloaded from the USGS for free. The performance of the Groundwater Flow Model has been tested in a variety of applications. Future applications, however, might reveal errors that were not detected in the test simulations. Users are requested to send notification of any errors found in this model documentation report or in the model program to the MODFLOW contact listed on the Web page. Updates might be made to both the report and to the model program. Users can check for updates on the MODFLOW Web page (https://doi.org/10.5066/F76Q1VQV).

\section{Acknowledgments}

The U.S. Geological Survey (USGS) Water Availability and Use Science Program provided financial support for the work documented herein. The authors are grateful for the constructive reviews provided by Steffen Mehl of California State University and Daniel Feinstein of the U.S. Geological Survey. The authors are also grateful for technical input provided by Arlen Harbaugh, Dave Pollock, and Richard Winston of the U.S. Geological Survey. 


\section{Contents}

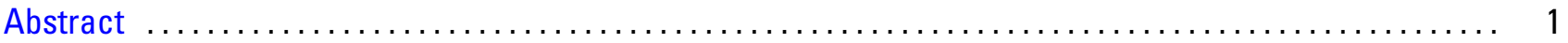

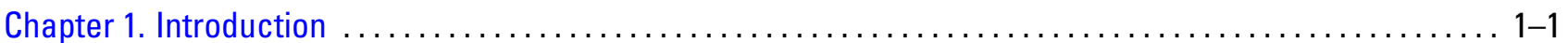

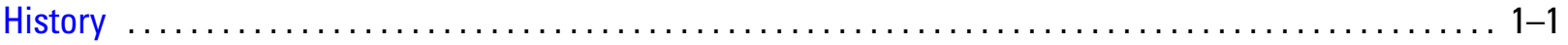

Overview of the MODFLOW 6 Framework and the Groundwater Flow Model $\ldots \ldots \ldots \ldots \ldots \ldots \ldots$ 1 3

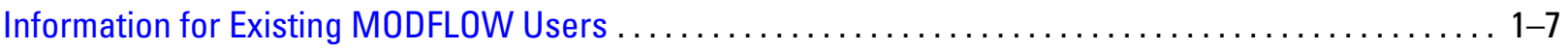

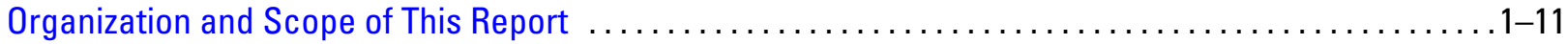

Chapter 2. Formulation and Solution of the Control-Volume Finite-Difference Equation $\ldots \ldots \ldots \ldots \ldots \ldots$ 2-1

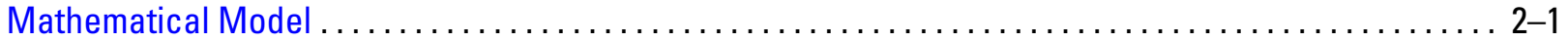

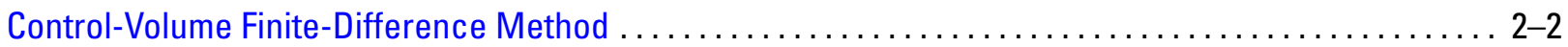

Structure of a Model Cell $\ldots \ldots \ldots \ldots \ldots \ldots \ldots \ldots \ldots \ldots \ldots \ldots \ldots \ldots \ldots \ldots \ldots \ldots \ldots \ldots \ldots \ldots \ldots \ldots \ldots \ldots \ldots \ldots \ldots, 2-2$

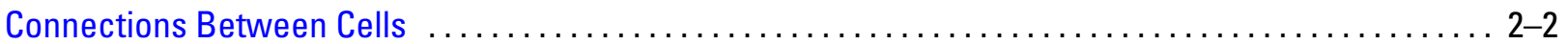

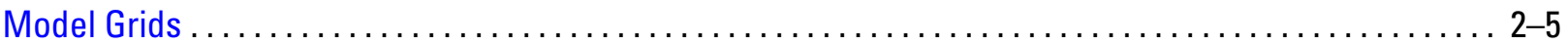

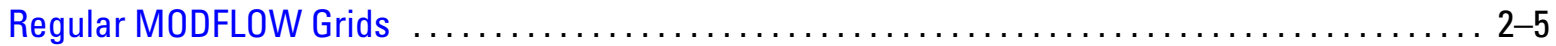

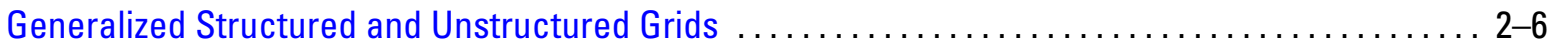

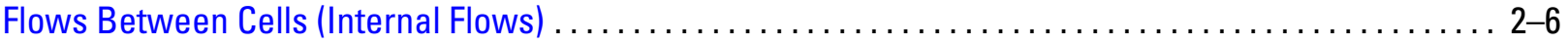

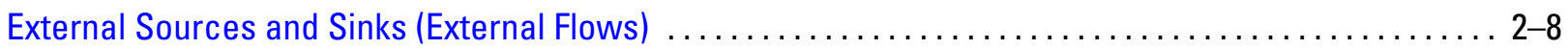

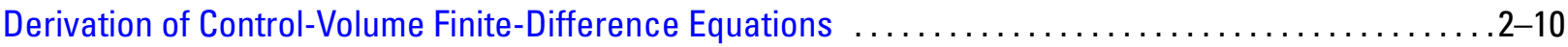

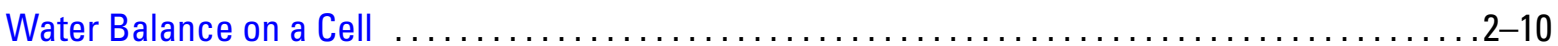

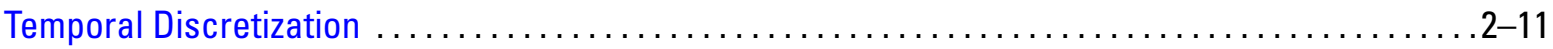

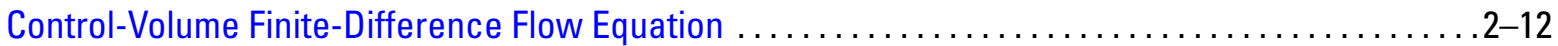

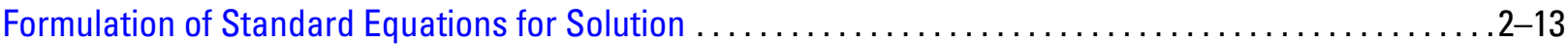

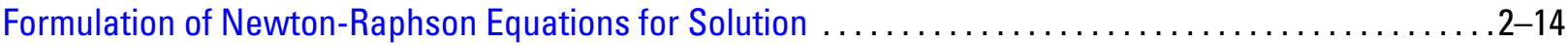

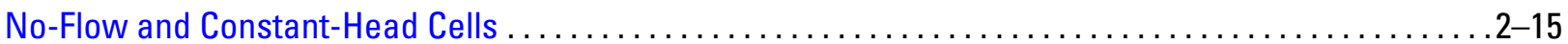

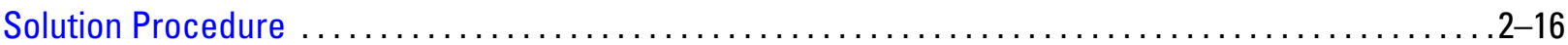

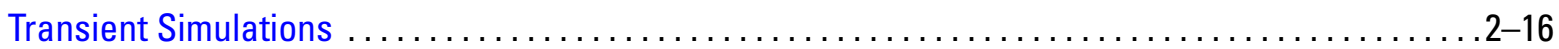

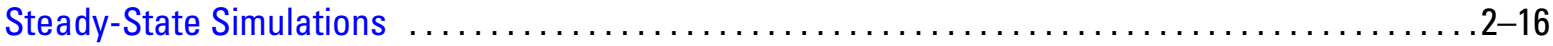

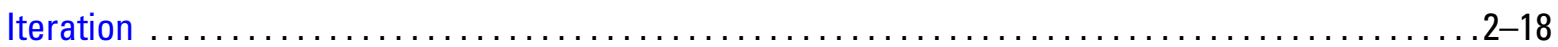

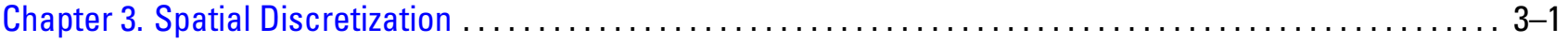

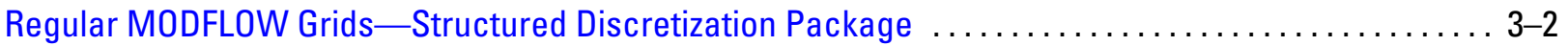

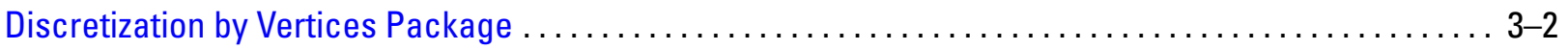

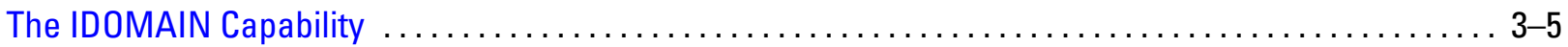

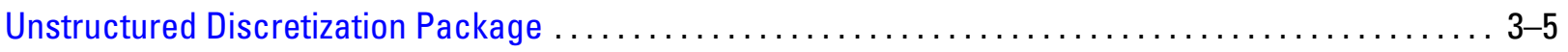

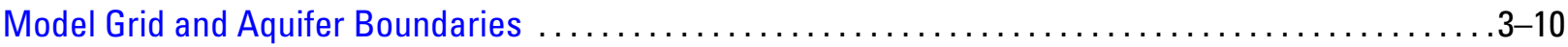

Considerations for Grid Design . . . . . . . . . . . . . . . . .

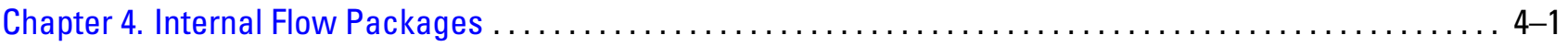

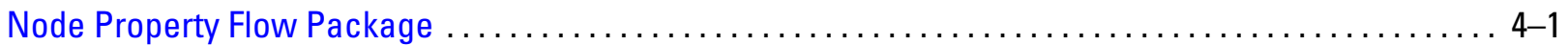

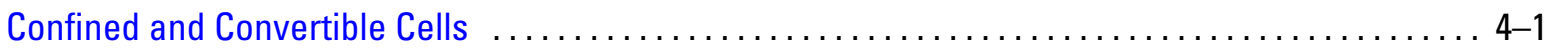

Saturated Cell Thickness ................................................. 4-2 


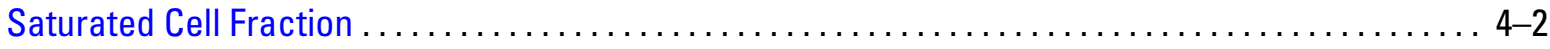

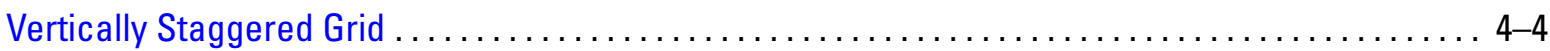

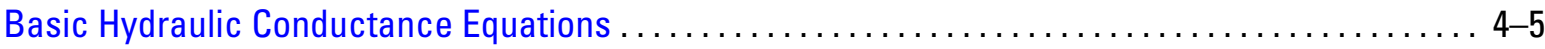

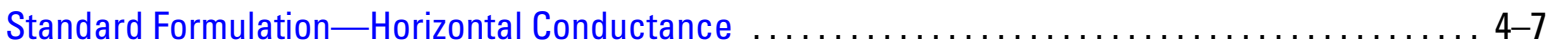

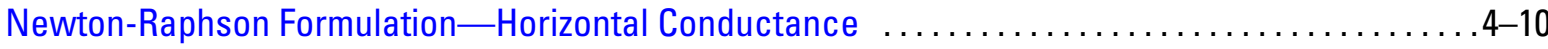

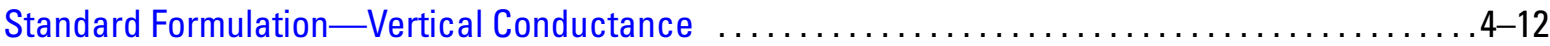

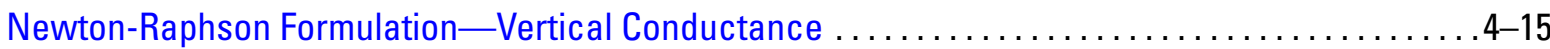

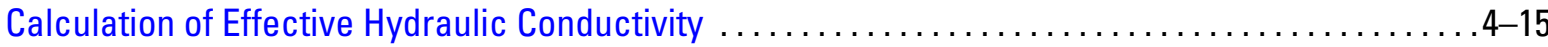

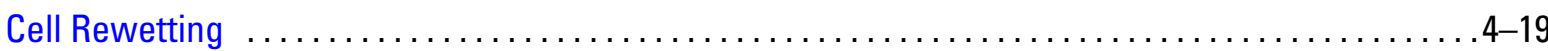

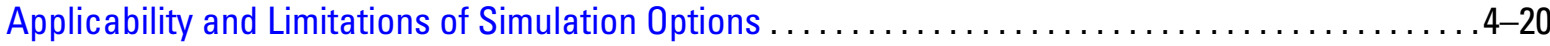

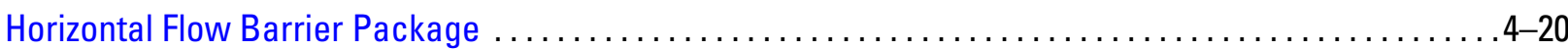

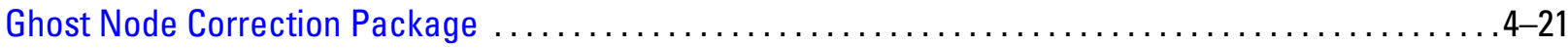

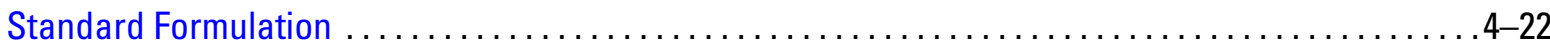

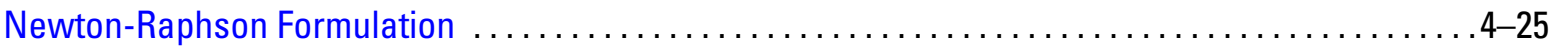

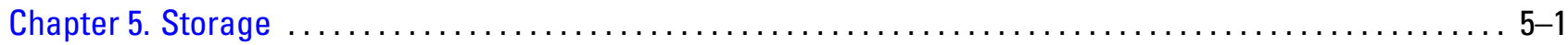

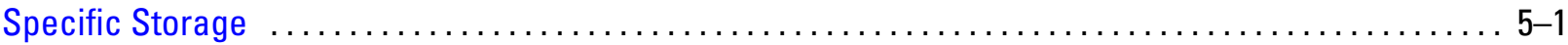

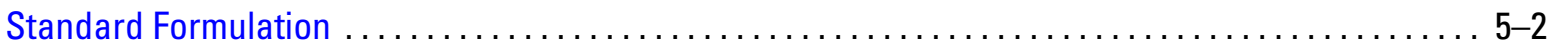

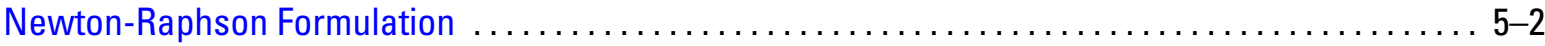

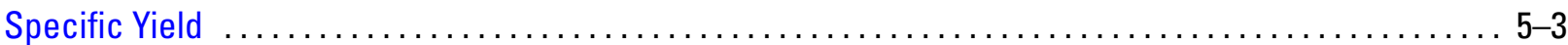

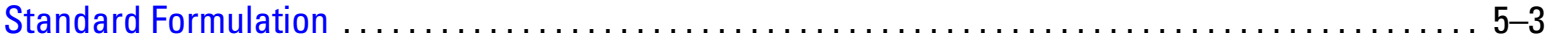

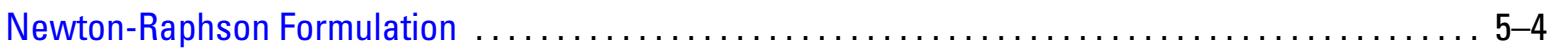

Chapter 6. Conceptualization and Implementation of Stress Packages $\ldots \ldots \ldots \ldots \ldots \ldots \ldots \ldots \ldots \ldots, 6$-1

Standard Formulation $\ldots \ldots \ldots \ldots \ldots \ldots \ldots \ldots \ldots \ldots \ldots \ldots \ldots \ldots \ldots \ldots \ldots \ldots \ldots \ldots \ldots \ldots, 1$

Head-Dependent Boundaries $\ldots \ldots \ldots \ldots \ldots \ldots \ldots \ldots \ldots \ldots \ldots \ldots \ldots \ldots \ldots \ldots \ldots, 6,1$

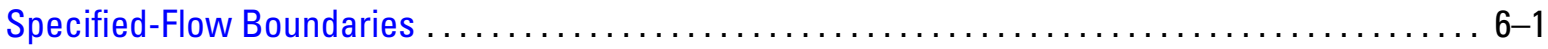

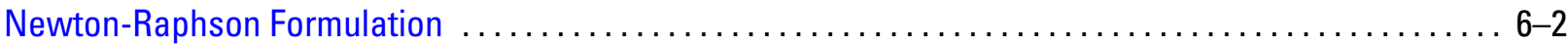

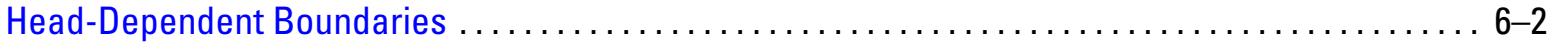

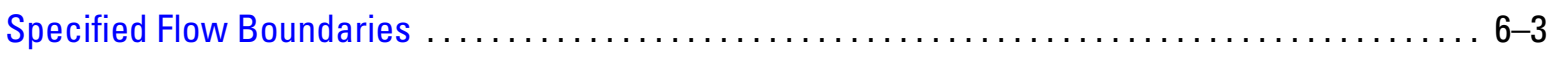

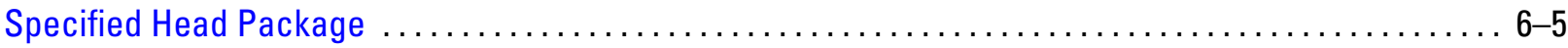

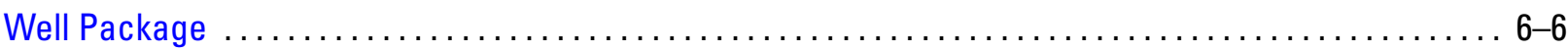

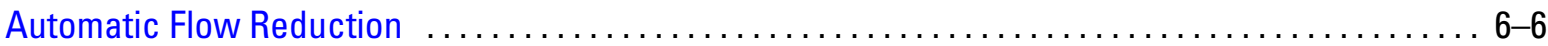

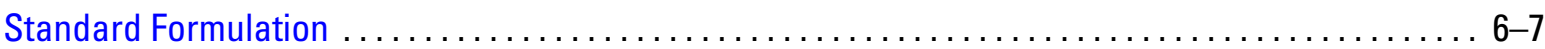

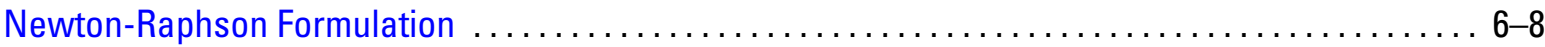

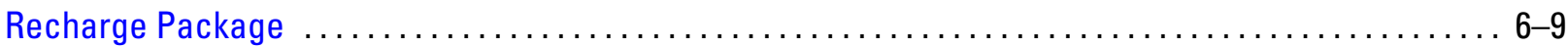

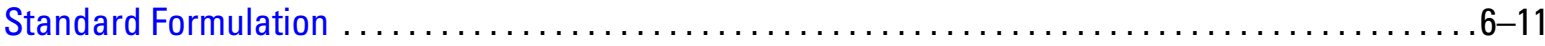

Newton-Raphson Formulation $\ldots \ldots \ldots \ldots \ldots \ldots \ldots \ldots \ldots \ldots \ldots \ldots \ldots \ldots \ldots, 6,11$

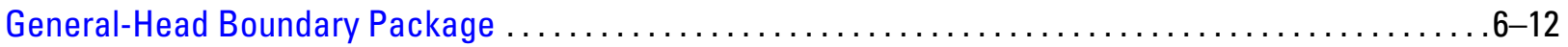

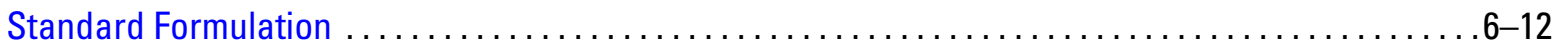

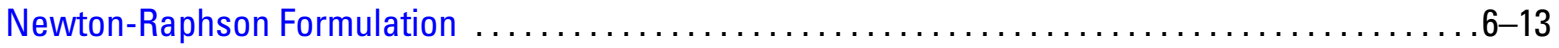

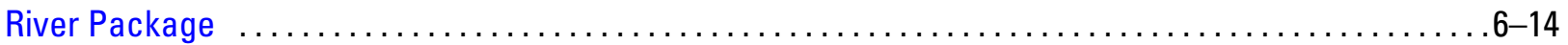

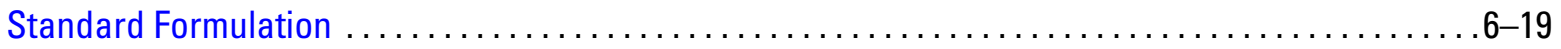

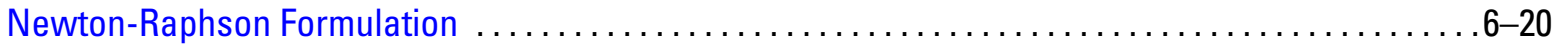




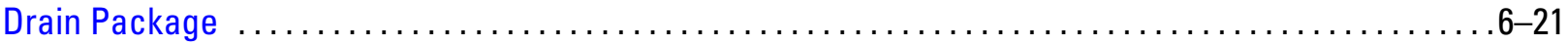

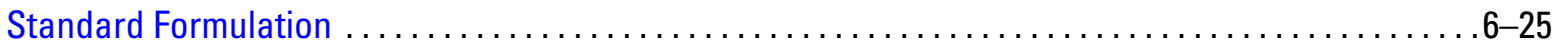

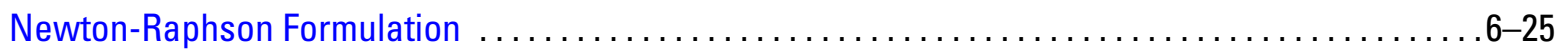

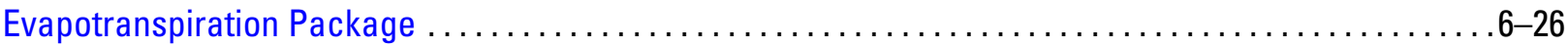

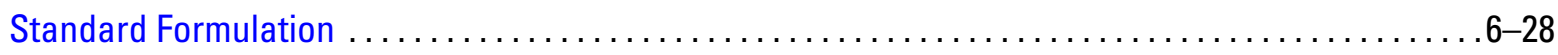

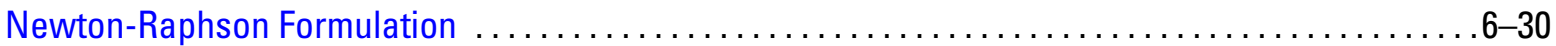

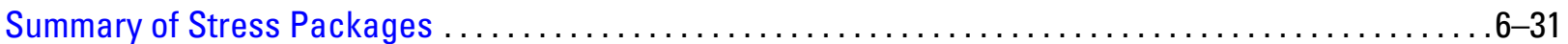

Chapter 7. Conceptualization and Implementation of Advanced Stress Packages $\ldots \ldots \ldots \ldots \ldots \ldots \ldots 7-1$

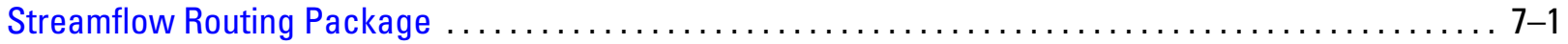

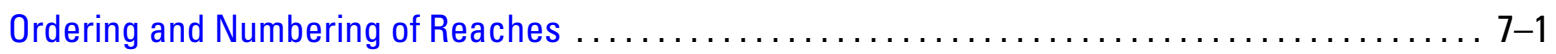

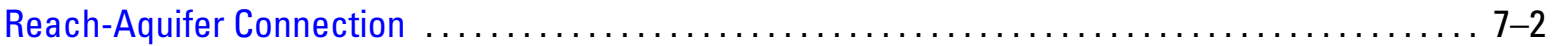

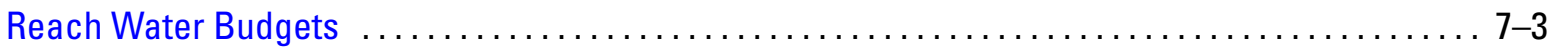

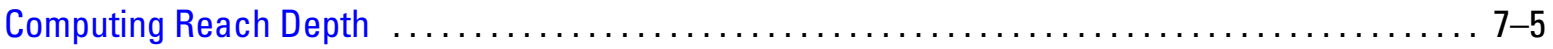

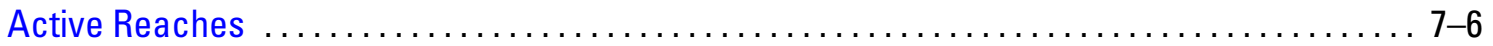

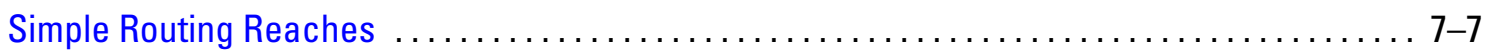

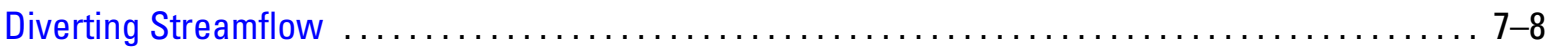

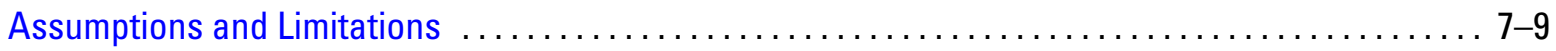

Incorporation of Leakage Into the Groundwater Flow Equation $\ldots \ldots \ldots \ldots \ldots \ldots \ldots \ldots \ldots \ldots .10$

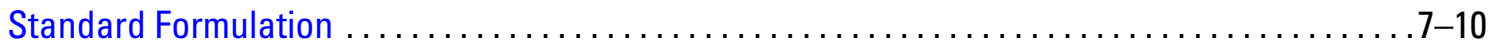

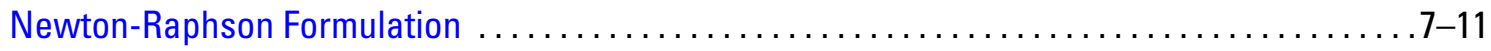

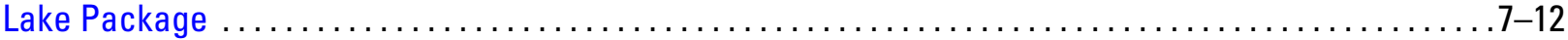

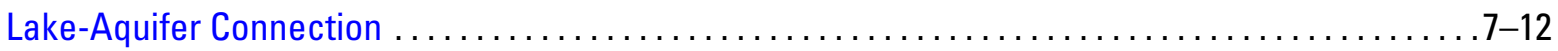

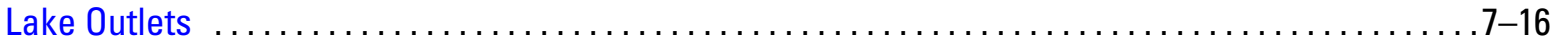

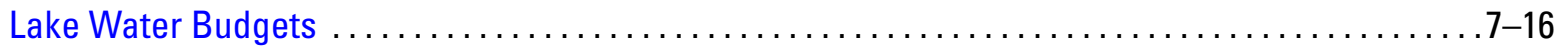

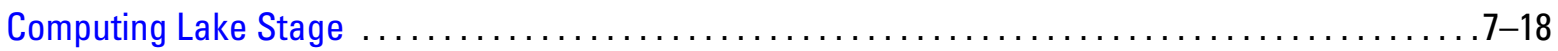

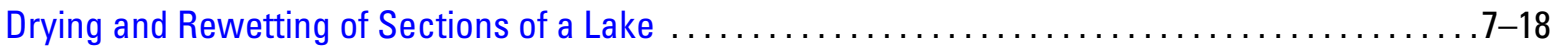

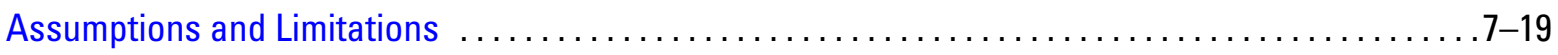

Incorporation of Leakage Into the Groundwater Flow Equation .....................

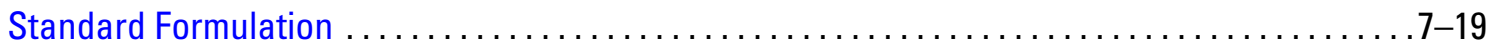

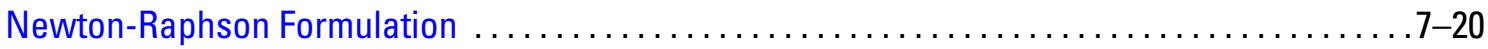

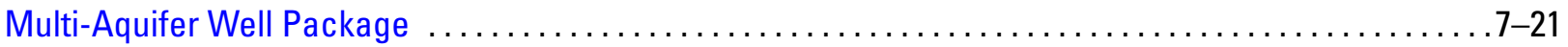

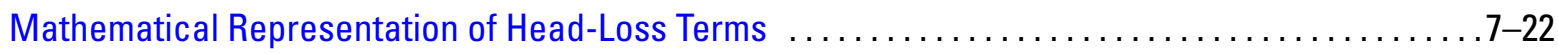

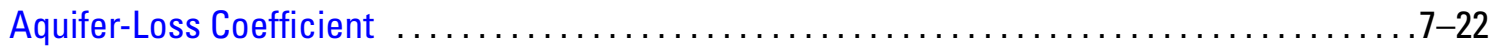

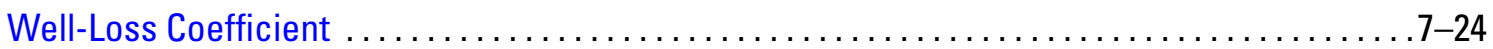

Aquifer- and Well-Loss Coefficient Conductance ..........................

Alternative Mathematical Representation of Head-Loss Terms $\ldots \ldots \ldots \ldots \ldots \ldots \ldots \ldots \ldots \ldots .25$

General Equations for Wells Connected to Multiple Grid Cells . .....................

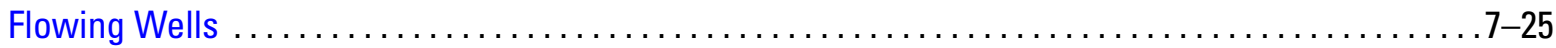

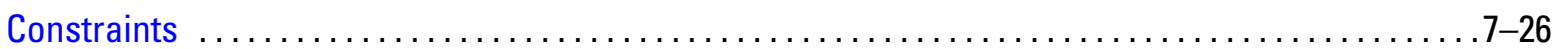

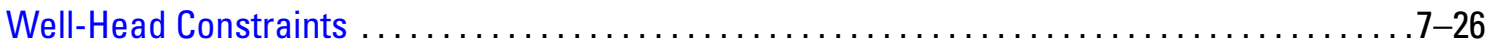

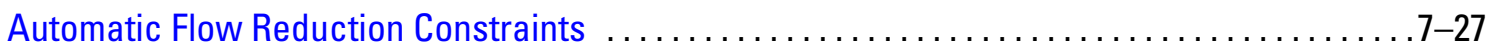

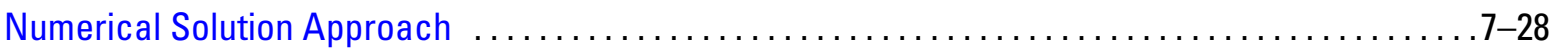

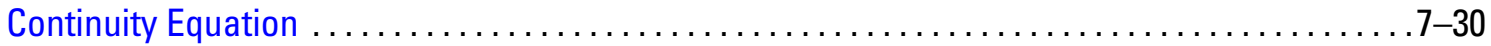


Incorporation of Multi-Aquifer Well Flow Terms Into the Groundwater Flow Equation $\ldots \ldots \ldots \ldots 7-30$

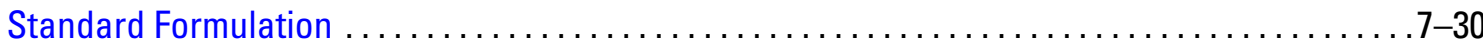

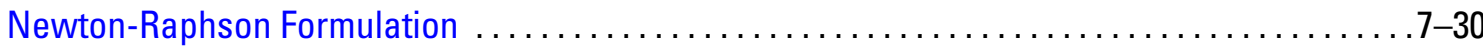

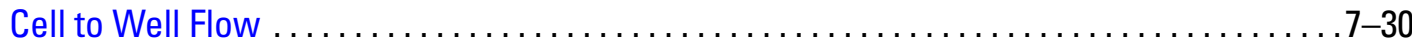

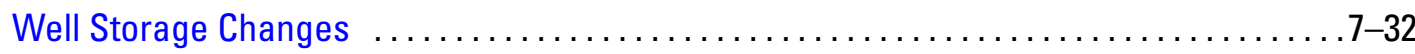

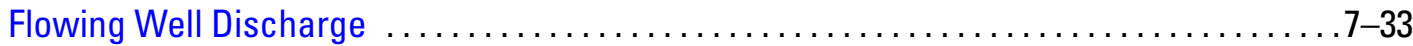

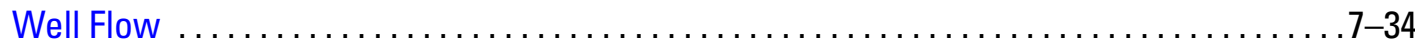

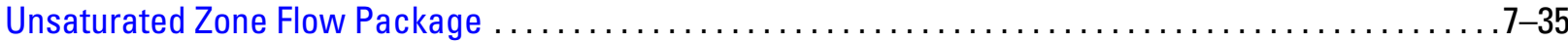

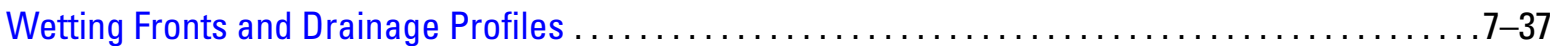

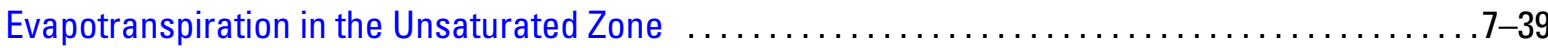

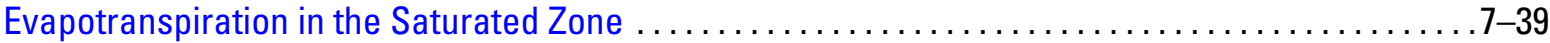

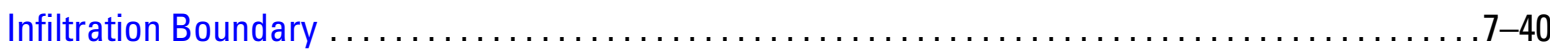

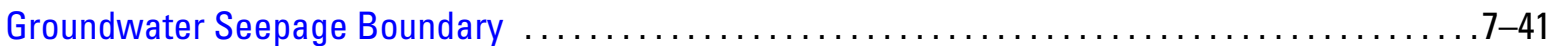

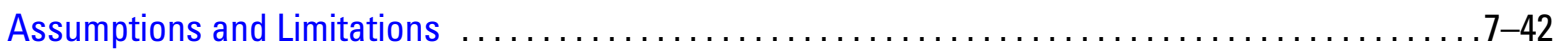

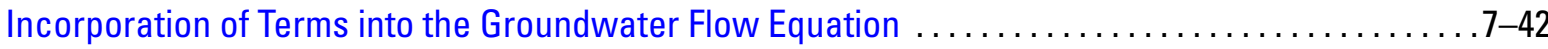

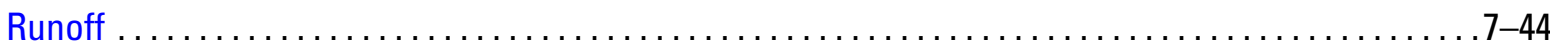

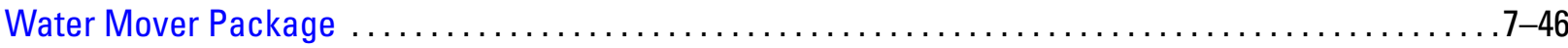

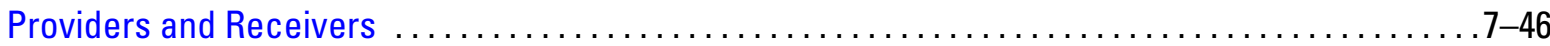

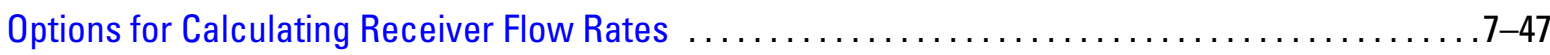

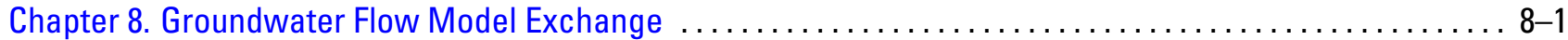

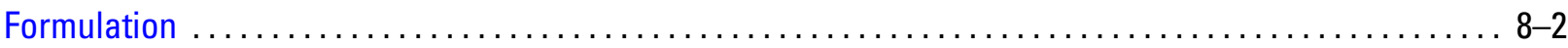

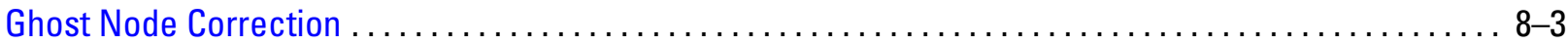

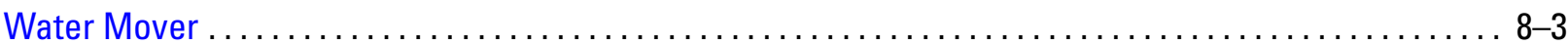

Limitations $\ldots \ldots \ldots \ldots \ldots \ldots \ldots \ldots \ldots \ldots \ldots \ldots \ldots \ldots \ldots \ldots \ldots \ldots \ldots \ldots \ldots \ldots \ldots \ldots \ldots \ldots, 3$

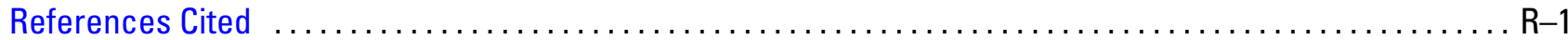

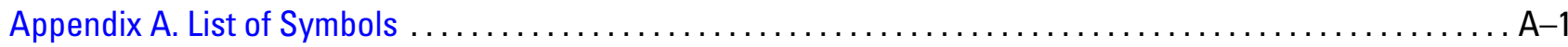

Appendix B. Implementation of the Groundwater Flow Model in the MODFLOW 6 Framework .......... B-1

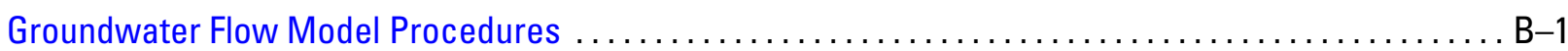

Packages . . . . . . . . . . . . . .

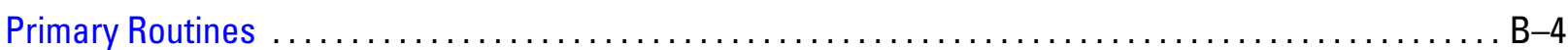

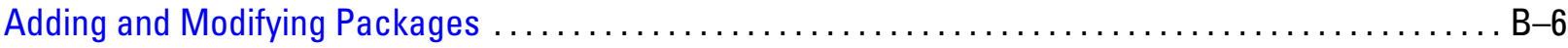

\section{Figures}

1-1. Diagram showing the MODFLOW 6 components for a simulation with a single Groundwater Flow Model . . . . . . . . . . . . . . . . . . . . . . . . . . . . . . . . . . . . . . . . . . . . . .

1-2. Diagram showing the MODFLOW 6 components for a simulation with two Groundwater Flow

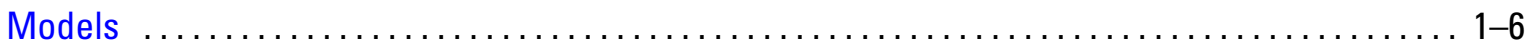

2-1. Diagram showing MODFLOW 6 model cells with nodes located at their centers. .......... 2-3

2-2. Diagram showing connections between the nodes of three model cells. $\ldots \ldots \ldots \ldots \ldots \ldots .2-3$

2-3. Diagram showing examples of two different types of cell connections. $\ldots \ldots \ldots \ldots \ldots \ldots \ldots 2-4$ 
2-4. Illustration showing a hypothetical aquifer system discretized using a regular MODFLOW

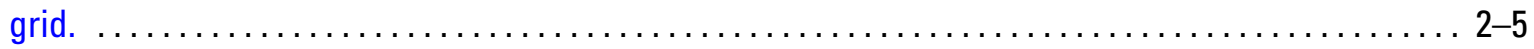

2-5. Illustration showing examples of different types of structured and unstructured grids. ...... 2-7

2-6. Diagram showing flow into cell $n$ from cell $m$. . . . . . . . . . . .

2-7. Diagram showing conceptual representation of leakage through a riverbed into a model

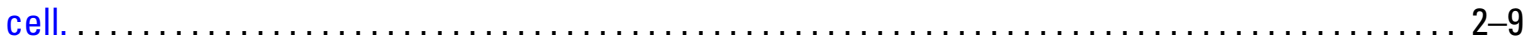

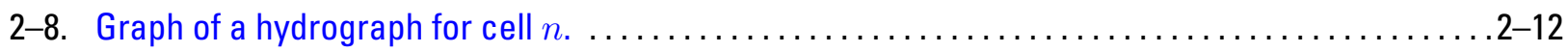

2-9. Diagram of a discretizated aquifer showing boundaries and cell designations. . . . . . . . $2-17$

2-10. Diagram showing iterative calculation of a head distribution. ......................

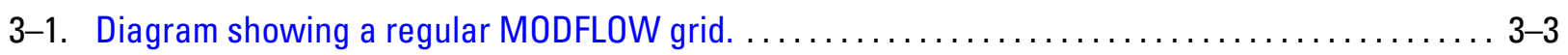

3-2. Diagram showing the vertices and cells defined using the Discretization by Vertices Pack-

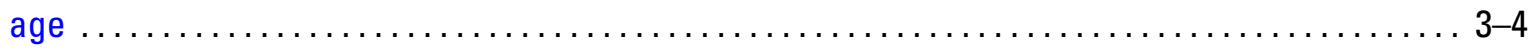

3-3. Diagram showing IDOMAIN variable in cross section for a single row or column. ........ 3-6

3-4. Diagram of a control-volume finite-difference grid showing connected cells and geometric

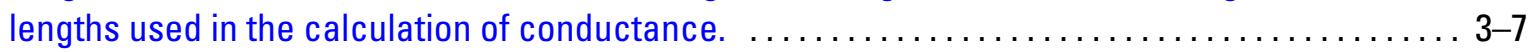

3-5. Diagram of cross section through a model grid used to demonstrate the concept of a horizontal connection and a vertically staggered horizontal connection. $\ldots \ldots \ldots \ldots \ldots \ldots \ldots .3-9$

3-6. Diagram showing the angle between the $x$-axis and an outward normal on the cell face. . ..... $3-10$

$3-7$. Diagram showing examples of convex and concave polygons. $\ldots \ldots \ldots \ldots \ldots \ldots \ldots \ldots \ldots \ldots \ldots \ldots$

3-8. Diagram showing different schemes of vertical discretization. $\ldots \ldots \ldots \ldots \ldots \ldots \ldots \ldots \ldots \ldots \ldots$

3-9. Diagram showing the possible pattern of flow in a cross section consisting of two high-

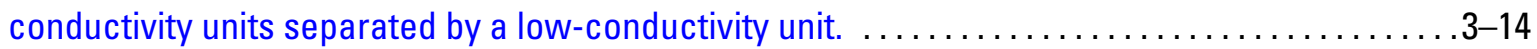

3-10. Diagram showing a cross section in which a low-conductivity unit is represented by six model layers. . . . . . . . . . . .

4-1. Graph showing combined quadratic and linear functions used to smooth the saturated fraction for a cell. . . . . . . . . . . . .

4-2. Diagram of a cross section showing cell $n$ connected to vertically staggered cells, including

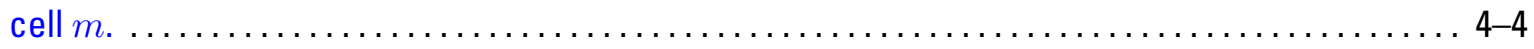

4-3. Diagram of prism of porous material used to illustrate Darcy's Law. . . . . . . . . . . . 4-5

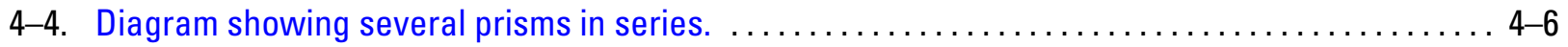

4-5. Diagram showing calculation of conductance between nodes using transmissivities and

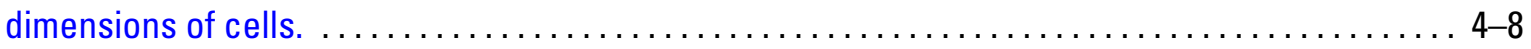

4-6. Diagram showing the situation in which a correction is required to limit the downward flow from cell $n$ into $m$, as a result of cell $m$ being partially dewatered. $\ldots \ldots \ldots \ldots \ldots \ldots \ldots \ldots$

4-7. Diagram showing anisotropic hydraulic conductivity ellipses for several different configura-

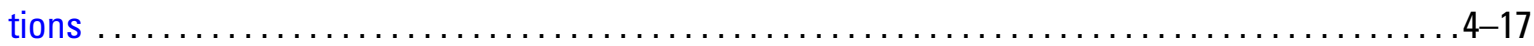

4-8. Diagram showing a low-permeability feature and its implementation in a model grid. ........4-21

4-9. Diagram showing a horizontal flow barrier separating cell $n$ from its horizontal neighbor cell

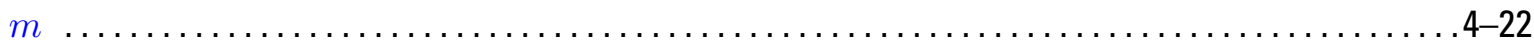

4-10. Diagram showing ghost node conceptualization for a nested grid. ................. $4-23$

6-1. Graph showing the cubic function used to smoothly reduce specified groundwater withdrawal rates to zero when a cell dewaters. 
6-2. Diagram showing the cells receiving recharge under the default option, which is based on applying recharge to the highest active cell, and the cells receiving recharge under the FIXED_CELL option. . . . . . . . . . . . . . .

6-3. Diagram illustrating principle of the General-Head Boundary Package . . . . . . . . . . .

6-4. Graph showing $Q G H B$ flow for a general-head boundary as a function of head, $h$, in cell $n$, where $H G H B$ is the source head. $\ldots \ldots \ldots \ldots \ldots \ldots \ldots \ldots \ldots \ldots \ldots \ldots \ldots \ldots \ldots \ldots \ldots \ldots \ldots \ldots \ldots, 13$

6-5. Diagram showing discretization of two rivers into reaches. $\ldots \ldots \ldots \ldots \ldots \ldots \ldots \ldots \ldots \ldots \ldots \ldots \ldots$

6-6. Diagram showing the interaction between a river and the underlying aquifer. $\ldots \ldots \ldots \ldots \ldots \ldots 6-15$

6-7. Diagram showing an idealized case of low-permeability riverbed sediments within an indi-

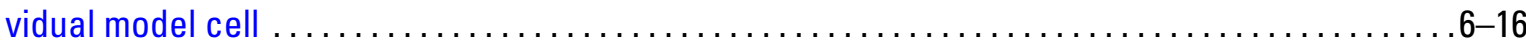

6-8. Diagram of cross sections showing relation between head at the bottom of the riverbed

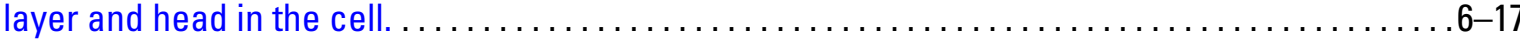

6-9. Graph showing $Q R I V$ as a function of head, $h$, in the cell, where $R B O T$ is the elevation of the bottom of the riverbed and $H R I V$ is the head in the river. . . . . . . . . . . . . . . . .

6-10. Diagram showing seepage from a river at unit hydraulic gradient. $\ldots \ldots \ldots \ldots \ldots \ldots \ldots \ldots \ldots \ldots$

6-11. Graph showing $Q D R N$ as a function of head, $h$, in a cell, where elevation of the drain is

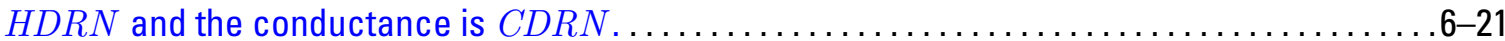

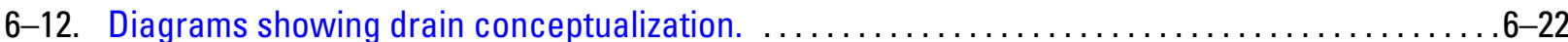

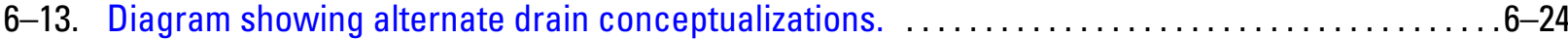

6-14. Graph of volumetric evapotranspiration (ET), $Q E T$, for a single ET segment, as a function of head, $h$, in a cell, where EXDP is the cutoff depth and SURF is the ET surface elevation.

6-15. Graphs showing volumetric evapotranspiration (ET) rate, $Q E T$, as a function of head for two situations in which the function in the variable interval is defined using multiple segments.

6-16. Diagram showing graphs of flow into or out of a model cell for the different stress packages.

7-1. Diagram of a simple stream network having six reaches shown within a regular MODFLOW grid consisting of three rows and three columns. $\ldots \ldots \ldots \ldots \ldots \ldots \ldots \ldots \ldots \ldots \ldots, 7-2$

7-2. Diagram showing several different stream reach configurations. $\ldots \ldots \ldots \ldots \ldots \ldots \ldots \ldots \ldots, 7$

7-3. Diagram showing an example of a reach-numbering scheme in relation to direction of flow

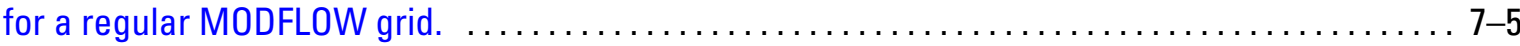

7-4. Diagram showing relation between the streamflow at the midpoint and end of a reach. ....... 7-6

7-5. Graph showing simulated stream depth with consecutive iterations of the mixed bisection-

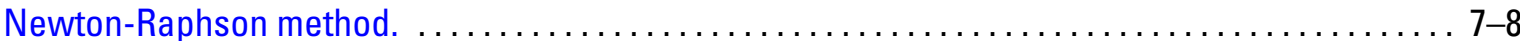

7-6. Diagram showing the concepts and length parameters used to calculate seepage between a lake and the aquifer. . . . . . . . . . . . . . . . . . . .

7-7. Diagram showing the approaches for calculating the saturation for horizontal lake connec-

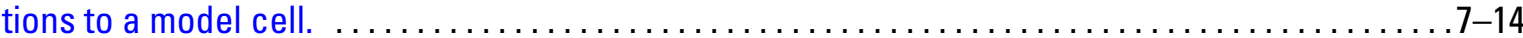

7-8. Diagram showing the effect of lake surface depth, SURFDEP, at the lake bottom on the wetted area of lake-aquifer connection for vertical connections in relation to the lake level or the water table in a model cell 
7-9. Diagram of a cross section showing flow patterns that can be induced by a multi-aquifer well. . . . . . . . . . . . . . .

7-10. Diagram of a well annulus (in plan view) showing well geometry and factors affecting the

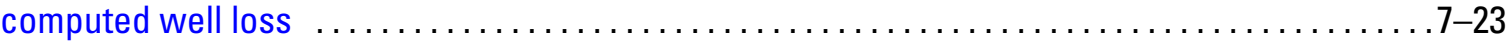

7-11. Diagram showing the relation between the computed withdrawal from a multi-aquifer well and the computed head in the well when user-defined well-head constraints are applied. ....7-27

7-12. Graph showing example of the cubic function used to smoothly reduce specified negative pumping rates to zero when the water level in a multi-aquifer well is less than a

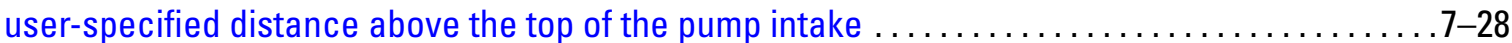

7-13. Graph showing an example of a multi-aquifer well coupled to multiple model cells, where the well is located at the center of a vertical sequence of model cells . . . . . . . . . . . . . $7-29$

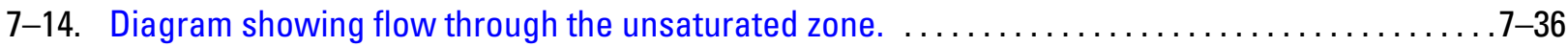

7-15. Diagram showing wetting fronts and drainage profiles in the unsaturated zone. .......... 7-38

7-16. Graph showing the evapotranspiration rate as a function of groundwater head for the

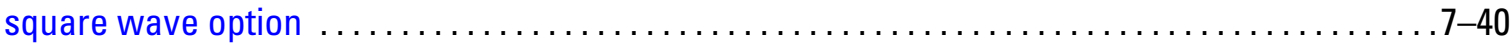

7-17. Diagram showing two configurations of elevation variations within a model cell that lead to

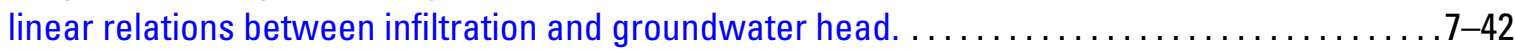

7-18. Graph showing rates of infiltration and groundwater discharge for different values of $\eta$ and how they vary over the user-specified surface depth, SURFDEP $\ldots \ldots \ldots \ldots \ldots \ldots \ldots .7-43$

7-19. Diagram of a model grid showing the Stream-Flow Routing (SFR) Package reach number for each Unsaturated Zone Flow (UZF) cell. . . . . . . . . . . . . . . . . . . .

8-1. Diagram showing model configurations where the Groundwater Flow Exchange (GWF-GWF) may be used to hydraulically connect two different Groundwater Flow Models. . . . . . . . . 8-1

B-1. Schematic diagram showing how primary procedures of the Groundwater Flow (GWF) Model are called from the Main Program and from the Iterative Model Solution

\section{Tables}

1-1. List of packages available for use with the Groundwater Flow Model $\ldots \ldots \ldots \ldots \ldots \ldots \ldots \ldots$. -4

3-1. Arrays that define cell connection properties for the unstructured Discretization Package

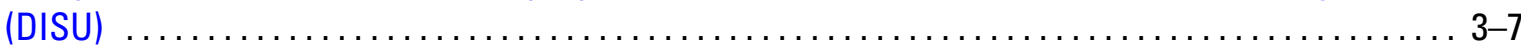

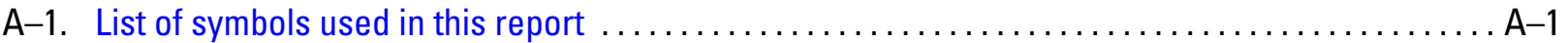

B-1. List of packages for the Groundwater Flow (GWF) Model $\ldots \ldots \ldots \ldots \ldots \ldots \ldots \ldots \ldots \ldots \ldots$

B-2. Groundwater Flow (GWF) Model primary routines classified by procedure and package ........ B-5 


\title{
Documentation for the MODFLOW 6 Groundwater Flow Model
}

\author{
By Christian D. Langevin, Joseph D. Hughes, Edward R. Banta, Richard G. Niswonger, \\ Sorab Panday, and Alden M. Provost
}

\section{Abstract}

This report documents the Groundwater Flow (GWF) Model for a new version of MODFLOW called MODFLOW 6. The GWF Model for MODFLOW 6 is based on a generalized control-volume finite-difference approach in which a cell can be hydraulically connected to any number of surrounding cells. Users can define the model grid using one of three discretization packages, including (1) a structured discretization package for defining regular MODFLOW grids consisting of layers, rows, and columns, (2) a discretization by vertices package for defining layered unstructured grids consisting of layers and cells, and (3) a general unstructured discretization package for defining flexible grids comprised of cells and their connection properties. For layered grids, a new capability is available for removing thin cells and vertically connecting cells overlying and underlying the thin cells. For complex problems involving water-table conditions, an optional NewtonRaphson formulation, based on the formulations in MODFLOW-NWT and MODFLOW-USG, can be activated. Use of the Newton-Raphson formulation will often improve model convergence and allow solutions to be obtained for difficult problems that cannot be solved using the traditional wetting and drying approach. The GWF Model is divided into "packages," as was done in previous MODFLOW versions. A package is the part of the model that deals with a single aspect of simulation. Packages included with the GWF Model include those related to internal calculations of groundwater flow (discretization, initial conditions, hydraulic conductance, and storage), stress packages (constant heads, wells, recharge, rivers, general head boundaries, drains, and evapotranspiration), and advanced stress packages (streamflow routing, lakes, multi-aquifer wells, and unsaturated zone flow). An additional package is also available for moving water available in one package into the individual features of the advanced stress packages. The GWF Model also has packages for obtaining and controlling output from the model. This report includes detailed explanations of physical and mathematical concepts on which the GWF Model and its packages are based.

Like its predecessors, MODFLOW 6 is based on a highly modular structure; however, this structure has been extended into an object-oriented framework. The framework includes a robust and generalized numerical solution object, which can be used to solve many different types of models. The numerical solution object has several different matrix preconditioning options as well as several methods for solving the linear system of equations. In this new framework, the GWF Model itself is an object as are each of the GWF Model packages. A benefit of the object-oriented structure is that multiple objects of the same type can be used in a single simulation. Thus, a single forward run with MODFLOW 6 may contain multiple GWF Models. GWF Models can be hydraulically connected using GWF-GWF Exchange objects. Connecting GWF models in different ways permits the user to utilize a local grid refinement strategy consisting of parent and child models or to couple adjacent GWF Models. An advantage of the approach implemented in MODFLOW 6 is that multiple models and their exchanges can be incorporated into a single numerical solution object. With this design, models can be tightly coupled at the matrix level. 


\section{Chapter 1. Introduction}

MODFLOW 6 is a new version of the U.S. Geological Survey (USGS) model commonly called MODFLOW. This report documents the Groundwater Flow (GWF) Model of MODFLOW 6. The primary objectives of the GWF Model are the same as for prior versions: The model can be readily understood and modified, is simple to use and maintain, is easily executed on a variety of computers with minimal changes, and is efficient with respect to computer memory and execution time.

MODFLOW has traditionally been a groundwater flow model. Simulations focus on the effects of wells, rivers, or other hydrologic stresses on an aquifer system. The scope of MODFLOW has expanded over the years to approximate surface-water flow, solute transport, and management optimization, for example. The following section describes the history related to MODFLOW development at the USGS, thereby providing the background for the developments presented in this report.

\section{History}

Prior to the development of MODFLOW, the two- and three-dimensional finite-difference models described by Trescott (1976), Trescott and Larson (1976), and Trescott and others (1976) were used extensively by the USGS and others for the computer simulation of groundwater flow. The first version of MODFLOW (McDonald and Harbaugh, 1984) was the result of the need to consolidate all the commonly used simulation capabilities into a single code that was easy to understand, use, and modify. This first version was developed between the spring of 1981 and the winter of 1983. That model code was originally called the USGS Modular Three-Dimensional Finite-Difference Ground-Water Flow Model; however, the model became known as MODFLOW several years later. This first MODFLOW version was developed using the Fortran 66 computer language.

Revised documentation was released in the report series Techniques of Water-Resources Investigations (TWRI) (McDonald and Harbaugh, 1988). The program was largely the same as the 1984 version, but small changes were made to make the code conform to Fortran 77 rather than to Fortran 66. This version of MODFLOW is called MODFLOW-88.

By the early 1990s, MODFLOW had become the most widely used groundwater flow model both within and outside the USGS. Many additions had been made to expand MODFLOW's capabilities. For example, more elaborate representation of the relation between streams and an aquifer was developed (Prudic, 1989). Leake and Prudic (1991) developed a package to represent subsidence. Two preconditioned conjugate-gradient packages were developed (Kuiper, 1987; Hill, 1990). An overall update to MODFLOW, called MODFLOW96, was published (Harbaugh and McDonald, 1996a,b). MODFLOW-96 was a relatively minor update primarily to improve ease of use by, for example, the introduction of a name file to list input and output files.

MODFLOW was originally conceived solely as a groundwater flow model. The original authors viewed the solution of additional related equations as something to be done in separate programs. An example of a related equation is a transport equation that uses flows computed from solution of the groundwater flow equation. Another example is parameter estimation, which solves an additional equation to compute optimal hydraulic parameters that result in the best match to real-world observations. By the late 1990s, there was a growing belief by many developers of modeling programs that combining such related capabilities into a single program promised to make development and use easier; therefore, the decision was made to broaden the scope of MODFLOW to allow capabilities, such as transport and parameter estimation, to be directly incorporated.

To facilitate the incorporation of related equations into MODFLOW, an expansion of the modular design was required. The result, which became MODFLOW-2000 (Harbaugh and others, 2000), was the addition of "Process," which is defined as parts of the code that solve a major equation or set of related equations. The part of the code that solves the groundwater flow equation became the Groundwater Flow (GWF) Process. 


\section{1-2 Documentation for the MODFLOW 6 Groundwater Flow Model}

Three processes-Observation, Sensitivity, and Parameter Estimation—aid calibration and model evaluation (Hill and others, 2000). Solution of the transport equation is the Groundwater Transport Process (Konikow and others, 1996) and the management of groundwater is the Ground-Water Management Process (Ahlfeld and others, 2005).

MODFLOW-2005 was similar in design to MODFLOW-2000, but expanded the concept of processes (Harbaugh, 2005). The primary change in MODFLOW-2005 was the incorporation of a different approach for managing internal data. Fortran modules were introduced to declare data that could be shared among subroutines. The Fortran modules allowed data to be shared without using subroutine arguments. As a result of using Fortran modules, a change in terminology for MODFLOW was made. MODFLOW subroutines were originally called modules in a generic sense. The generic term module was eliminated and replaced by the term subroutine. MODFLOW-2005 was written in the Fortran 90 programming language.

Following the release of MODFLOW-2005, there was rapid development and publication of numerous MODFLOW variants. Most of the variants used MODFLOW-2005 as their core. The following list summarizes selected MODFLOW variants published by the USGS since 2005:

- MODFLOW-FMP includes the ability to simulate the effects of farm irrigation on groundwater supplies (Schmid and others, 2006);

- SEAWAT couples MODFLOW with MT3DMS (Zheng and Wang, 1999) for simulation of variabledensity groundwater flow (Langevin and others, 2008);

- GSFLOW couples the PRMS hydrologic model (Markstrom and others, 2015) with MODFLOW (Markstrom and others, 2008);

- MODFLOW-CFP includes the Conduit Flow Process (CFP) developed to simulate non-Darcian flow in karst aquifers (Shoemaker and others, 2008);

- MODFLOW-GWM optimizes groundwater management strategies (Ahlfeld and others, 2009);

- MODFLOW-CDSS was developed for the State of Colorado as part of a decision support system to add a capability to partition stress packages (Banta, 2011);

- MODFLOW-NWT provides a robust approach for simulating complex water table conditions (Niswonger and others, 2011);

- MODFLOW-SWR includes a surface-water model (Hughes and others, 2012) for simulating coupled groundwater and surface-water interactions;

- MODFLOW-LGR allows multiple groundwater flow models to be coupled within a local grid refinement framework (Mehl and Hill, 2013);

- MODFLOW-USG is an unstructured grid version of MODFLOW that allows flexible grid geometries (Panday and others, 2013), and

- MODFLOW-OWHM simulates water movement under the supply-and-demand conditions characteristic of agricultural environments (Hanson and others, 2014).

This list of MODFLOW variants exemplifies the breadth of hydrologic problems requiring numerical simulation, and shows how the underlying MODFLOW formulation has been expanded over the years to include new formulations and numerical approaches. It is also clear from this list that MODFLOW has been extended into more than just a groundwater flow model. The addition of solute and heat transport, surface-water flow, landscape hydrologic processes, and flow within pipes and conduits adds complexities that were not considered 
when MODFLOW was originally designed. For many of these MODFLOW variants, it was not a straightforward process to implement the desired functionality. In some cases, the original design concepts of MODFLOW, such as package independence and the ability to be easily understood, were sacrificed.

\section{Overview of the MODFLOW 6 Framework and the Groundwater Flow Model}

The goals for MODFLOW 6, as for all prior versions of MODFLOW, can be easily stated: The program is easy to understand, use, enhance, and modify. An additional goal for MODFLOW 6 is development of a new MODFLOW framework that supports many of the capabilities that have been implemented in the MODFLOW variants shown in the previous section, such as unstructured grids, local grid refinement, and coupling to other hydrologic processes. To accomplish these goals, MODFLOW 6 was designed from the bottom up with a special emphasis on designing a program that is logical and organized and that can be extended in the future. For example, MODFLOW 6 uses an updated modular structure wherein similar program functions are grouped together into objects, and specific computational and hydrologic options are constructed in such a manner so that each option is independent of other options. Because of this structure, new capabilities and options can be added without the necessity of changing existing capabilities and options. The MODFLOW 6 framework is described in a companion report by Hughes and others (2017).

An important design decision in the development of MODFLOW 6 was to formalize the concept of a "model." A model is that part of the program that solves a hydrologic process. The version of MODFLOW 6 described in this report supports one type of model - the GWF Model. Other models may be added in the future, such as a groundwater transport model, a surface-water model, and a pipe network model, for example. Underlying MODFLOW 6 is a framework that allows developers to add new models and the interactions between models. A key feature of the new MODFLOW 6 framework is the ability to solve multiple, tightly coupled, numerical models in a single system of equations. These may be multiple models of the same type or of different types. For example, this capability can be used for local grid refinement applications in which multiple GWF Models are solved simultaneously within a single matrix solution.

Object-oriented programming is currently the standard approach for developing sophisticated computer programs. One benefit of the object-oriented approach is that individual software components, called objects, can be developed and tested independently. The functionality of these objects can then be made available for other parts of the program to use, without the need for developers of those parts to understand the underlying object details. Since its early inception, MODFLOW has been largely based on the same fundamental principles that now define object-oriented programming. For example, the notion that packages are independent from other packages has been a guiding MODFLOW principle. Formal implementation of object-oriented programming with the Fortran language was not available until the 2003 standard (International Standards Organization, 2004) was released. MODFLOW 6 uses the object-oriented features of Fortran 2003 to define the framework for simulating multiple hydrologic processes and their interactions. Some features from the Fortran 2008 (International Standards Organization, 2010) language also are used. Programs written in standard Fortran are highly portable. Use of non-standard features has been carefully avoided so that MODFLOW 6 will run, without modification, on most computers.

The GWF Model described in this report is divided into "packages," as was done in previous MODFLOW versions. A package is the part of the model that deals with a single aspect of simulation. For example, the Well Package simulates the effect of wells, and the River Package simulates the effect of rivers. The GWF Model contains many packages and options that the user may or may not have occasion to use. There are three types of hydrologic packages. The first type is the Hydrologic/Internal Package, which is an internal flow package that simulates flow between adjacent cells or handles storage changes for all the model cells. The second type is the Hydrologic/Stress Package, which simulates a simplified and individual kind of stress (such as rivers, wells, and recharge). Lastly, the Hydrologic/Advanced Stress Package simulates more advanced 


\section{1-4 Documentation for the MODFLOW 6 Groundwater Flow Model}

stresses. The more advanced stresses typically involve solving some form of a water budget equation for the stress features, such as a stream, lake, multi-aquifer well, or unsaturated zone.

Table 1-1 lists the various packages of the GWF Model that are documented in this publication, the threecharacter abbreviation used for each package, and the package category. The Hydrologic/Internal Packages calculate terms required to solve the groundwater flow equation for each model cell or store the information needed to calculate these terms. The Discretization (DIS, DISV, and DISU) Packages calculate or manage cell surface areas and volumes, and the geometric properties of the connections between cells. The Initial Conditions (IC) Package reads the starting heads for a GWF Model. The Node-Property Flow (NPF) Package calculates hydraulic conductance between adjacent cells, manages wetting and drying of cells, and calculates the flow between adjacent cells. The Horizontal Flow Barrier (HFB) Package is a supplementary internal flow package that works with the NPF Package to modify conductances to simulate a barrier between horizontally adjacent nodes. The Ghost Node Correction (GNC) Package is a supplementary internal flow package that works with NPF to improve the accuracy of flow calculations for some grid types. The Storage (STO) Package calculates the change in water volume that occurs over a time step. Only one instance of each of the Hydrologic/Internal Packages listed in table 1-1 can be used for a GWF Model; for example, there can be only one STO Package used for a GWF Model.

Table 1-1. List of packages available for use with the Groundwater Flow Model.

\begin{tabular}{lll}
\hline \hline Package Name & Abbreviation & Package Category \\
\hline Discretization & DIS, DISV, or DISU & Hydrologic/Internal \\
Initial Conditions & IC & Hydrologic/Internal \\
Node Property Flow & NPF & Hydrologic/Internal \\
Horizontal Flow Barrier & HFB & Hydrologic/Internal \\
Ghost Node Correction & GNC & Hydrologic/Internal \\
Storage & STO & Hydrologic/Internal \\
\hline Specified Head & CHD & Hydrologic/Stress \\
Well & WEL & Hydrologic/Stress \\
Recharge & RCH & Hydrologic/Stress \\
River & RIV & Hydrologic/Stress \\
General-Head Boundary & GHB & Hydrologic/Stress \\
Drain & DRN & Hydrologic/Stress \\
Evapotranspiration & EVT & Hydrologic/Stress \\
\hline Stream-Flow Routing & SFR & Hydrologic/Advanced Stress \\
Lake & LAK & Hydrologic/Advanced Stress \\
Multi-Aquifer Well & MAW & Hydrologic/Advanced Stress \\
Unsaturated Zone Flow & UZF & Hydrologic/Advanced Stress \\
Water Mover & MVR & Hydrologic/Advanced Stress \\
\hline Model Observations & OBS & Output \\
Output Control & OC & Output \\
\hline
\end{tabular}

Each Hydrologic/Stress Package formulates the coefficients describing a particular external or boundary flow; for example, the River Package calculates the coefficients describing flow between a cell and a surface river. Packages in the Hydrologic/Stress category include: CHD, WEL, RCH, RIV, GHB, DRN, and EVT. These seven packages are described by Harbaugh (2005) and provide core MODFLOW functionality that has been available in most previous MODFLOW releases. The MAW, SFR, UZF, and MVR Packages 


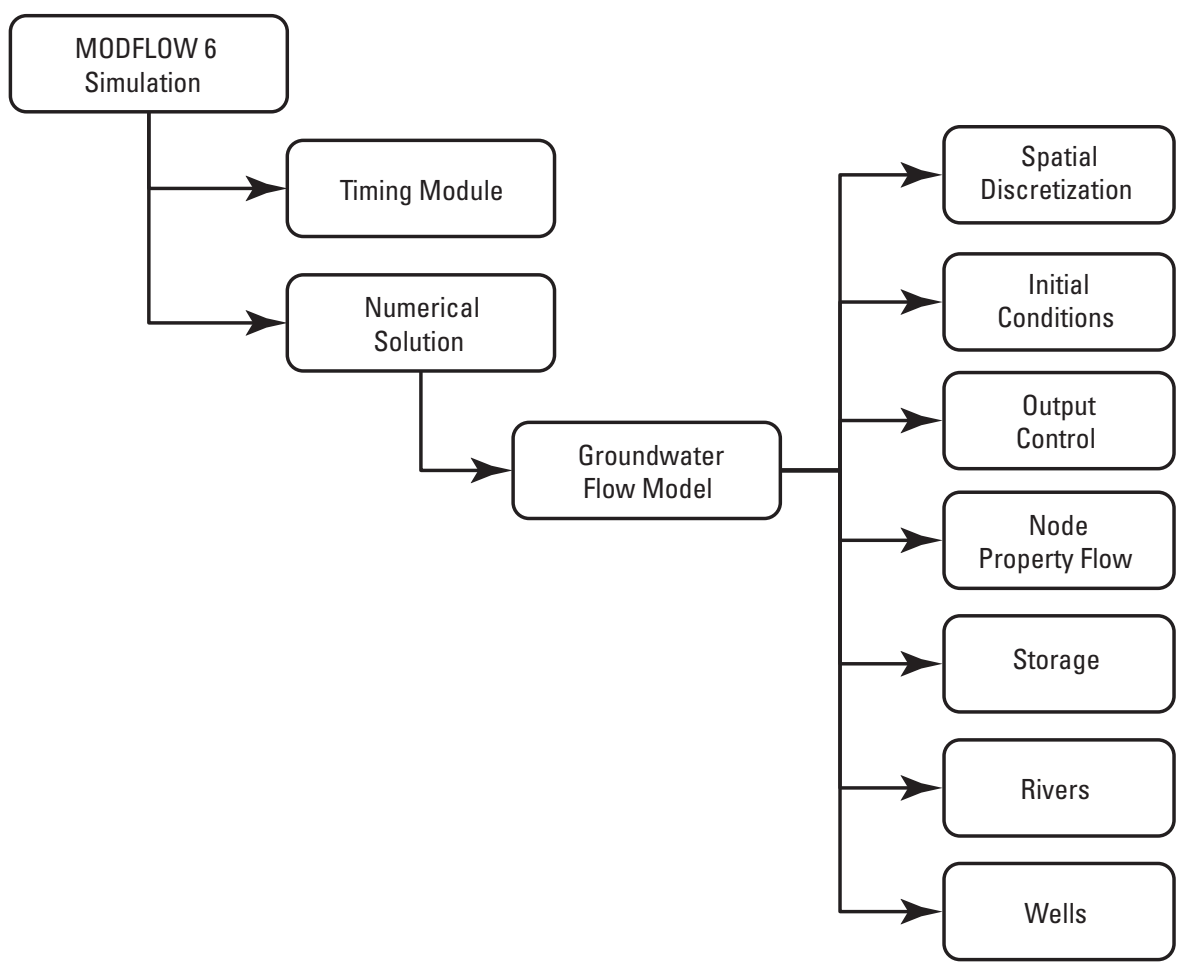

Figure 1-1. Diagram showing the MODFLOW 6 components for a simulation with a single Groundwater Flow Model.

are more complicated than the seven core stress packages. For this reason, they are grouped into the Hydrologic/Advanced Stress category. A unique capability of the GWF Model in MODFLOW 6 is that multiple Hydrologic/Stress and Hydrologic/Advanced Stress packages of the same type can be included in a single GWF Model. This multi-package capability is also available in MODFLOW-CDSS (Banta, 2011). Another new feature available for the stress packages is use of time-series records to specify certain types of stress input. The time-series capability is described in appendix B.

The only packages that do not fit into the hydrologic categories are the Observation (OBS) and the Output Control (OC) Packages, which manage the printing and saving of GWF Model results to output files. These packages are grouped in the Output category shown in table 1-1. A GWF Model can contain at most one instance of an OBS Package and one instance of an OC Package.

To support the multi-model capability in the MODFLOW 6 framework, information about simulation timing is controlled at the simulation level. Thus, the lengths of simulation time steps are determined and applied for all models. The simulation also needs information about the models that are included in the simulation, how the models interact with one another, and how the models and their interactions are solved. The hierarchy and simulation components for MODFLOW 6 are demonstrated in figure 1-1 for a simulation with a single groundwater flow model. In this example, the simulation consists of a Timing Module, a Numerical Solution, and a GWF Model. Beneath the GWF Model are individual packages, which describe the hydrogeologic processes that are simulated. When there is more than one GWF Model, as shown in figure 1-2, then an Exchange can be used to hydraulically connect the two models. Exchanges for connecting two GWF Models are labeled with the GWF-GWF identifier. Users can specify as many GWF-GWF Exchanges as necessary to connect any number of GWF Models in the proper configuration. When multiple GWF models are coupled, they are coupled at the matrix level, which allows them to be solved as efficiently as if they were built into a single unstructured grid. 


\section{1-6 Documentation for the MODFLOW 6 Groundwater Flow Model}

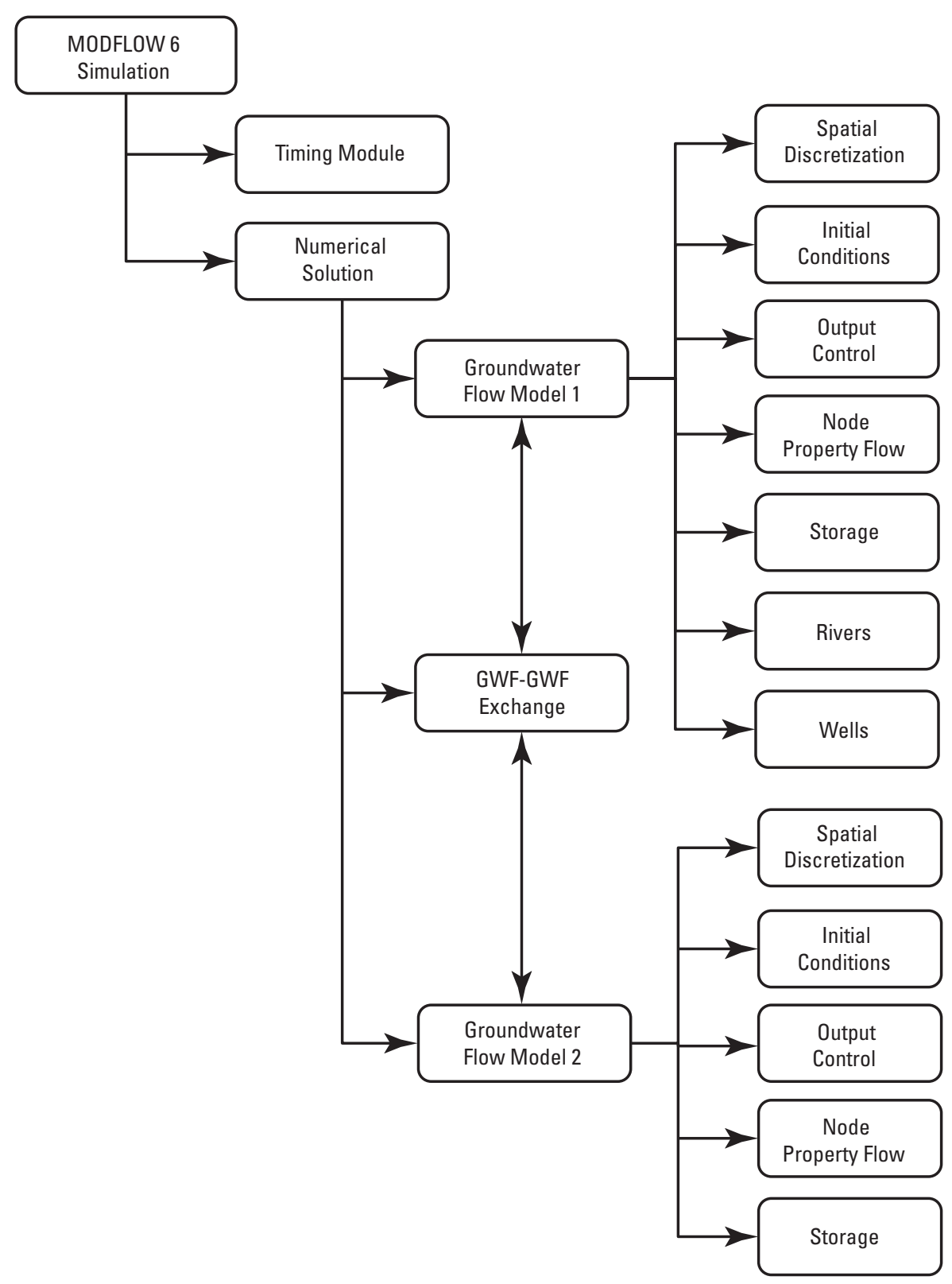

Figure 1-2. Diagram showing the MODFLOW 6 components for a simulation with two Groundwater Flow Models. 


\section{Information for Existing MODFLOW Users}

MODFLOW 6 contains most of the functionality of MODFLOW-2005, MODFLOW-NWT, MODFLOWUSG, and MODFLOW-LGR. To the existing MODFLOW user, however, MODFLOW 6 will feel different from previous MODFLOW versions. Some packages have been divided, renamed, or removed, and some capabilities, which previously caused confusion or were implemented due to computer memory limitations, are no longer supported (for example, "quasi-3d confining units" are not supported in the GWF Model). The form of the input files for MODFLOW 6 is different from previous MODFLOW versions in that input files are now divided into blocks, and keywords are used to specify options and input variables. The Fortran source code also looks different from previous versions. It is written in an object-oriented style that makes widespread use of Fortran modules, derived types, and type-bound procedures. Extensive testing was used as part of the development process to ensure that MODFLOW 6 simulation results are identical to the results from previous MODFLOW versions. In some cases, it was not possible to exactly replicate the simulation results from previous MODFLOW versions. In those cases, the differences could be explained by an option that is no longer supported, or because of slight differences in the underlying formulation.

The following list summarizes the major differences between the GWF Model in MODFLOW 6 and previous versions of MODFLOW. This list is intended for those with a general understanding of the capabilities in previous versions of MODFLOW.

1. The MODFLOW 6 framework extends the MODFLOW-USG concept and formalizes the way in which other models and packages can expand the underlying matrix equations to solve hydrologic processes in a tightly coupled manner (Hughes and others, 2017). The GWF Model in MODFLOW 6 is based on a generic unstructured design, patterned after the MODFLOW-USG design. This allows a GWF Model cell to be connected at the matrix level to any number of adjacent cells. A GWF Model cell can also be connected to a cell in another GWF Model or to other types of hydrologic features.

2. The GWF Model in MODFLOW 6 supports three alternative input packages for specifying the grid used to discretize the groundwater system.

- The Discretization (DIS) Package defines a grid based on layers, rows, and columns. In this report, this type of grid is referred to as a "regular MODFLOW grid" because it corresponds to traditional MODFLOW grids. An interior cell in a regular MODFLOW grid is connected to four adjacent cells in the same layer, to one overlying cell, and to one underlying cell.

- The Discretization by Vertices (DISV) Package defines a grid using a list of $(x, y)$ vertex pairs and the number of layers. A list of vertices is provided by the user to define a two-dimensional horizontal grid in plan view. This list of vertices may define a regular MODFLOW grid, or they may define more complex grids, such as grids consisting of triangles, hexagons, or Voronoi polygons, for example. This same two-dimensional horizontal grid applies to each layer in the model. Cells defined using the DISV Package are referenced by layer number and by the cell number within the horizontal grid. Within a layer, a cell may be horizontally connected to any number of surrounding cells in that layer. In the vertical direction a cell can be connected to only one overlying cell and only one underlying cell. Grids defined with the DISV Package are considered to be unstructured.

- The unstructured Discretization (DISU) Package is the most flexible of the three packages and is patterned after the unstructured grid implemented in MODFLOW-USG. For each cell, the user specifies a list of connected cells and the connection properties. When the DISU Package is used, cells are referenced only by their cell number; unlike the MODFLOW-USG approach, there is no concept of a layer in the DISU Package in MODFLOW 6, but cells may still overlie or underlie one another. 


\section{1-8 Documentation for the MODFLOW 6 Groundwater Flow Model}

3. For the layered grid types supported in the GWF Model (DIS and DISV), cells can be permanently excluded from the grid for the simulation. Input values (such as hydraulic conductivity) are still required for these excluded cells, and the program will write special codes or zero values for output, but the program does not allocate memory or store values for excluded cells during run time. In this case, the matrix equations are formulated for a reduced system in which only the included cells are numbered. Users can also mark excluded cells as "vertical pass-through cells." When these vertical pass-through cells are encountered, the program connects the cells overlying and underlying the pass-through cell. This capability allows "pinched" cells to be removed from the solution. These options to exclude cells or exclude them as pass-through cells are available for the DIS and DISV Packages through specification of the IDOMAIN array; the IDOMAIN capability is not available for the DISU Package.

4. There is no longer a Basic Package input file. Initial head values are specified using an Initial Conditions (IC) Package, and constant heads are specified using the Time Varying Specified Head (CHD) Package. Cells that are permanently excluded from the simulation can be eliminated using the IDOMAIN capability entered through the DIS or DISV Packages. For a cell that may transition from inactive ("dry") to active ("wet") during a simulation, the user can start the cell as inactive by assigning an initial head below the cell bottom.

5. The Newton-Raphson formulations and accompanying upstream weighting schemes implemented in MODFLOW-NWT and MODFLOW-USG for handling dry or nearly dry cells have been synthesized into a single formulation. The Newton-Raphson formulation in the GWF Model for MODFLOW 6 remains an optional alternative to the standard formulation used in most previous MODFLOW versions. Much of this report is focused on systematically explaining standard and Newton-Raphson formulations for the GWF Model and its packages.

6. Information on temporal discretization, such as number of stress periods, period lengths, number of time steps, and time step multipliers, is specified at the simulation level, rather than for an individual model. This information is provided in the Timing Module, which controls the temporal discretization and applies to all models within a simulation. The Timing Module is part of the MODFLOW 6 framework and is described separately in Hughes and others (2017).

7. Aquifer properties used to calculate hydraulic conductance are specified in the Node Property Flow (NPF) Package. In MODFLOW 6, the NPF Package calculates intercell conductance values, manages cell wetting and drying, and adds Newton-Raphson terms for intercell flow expressions. The NPF Package allows individual cells to be designated as confined or convertible; this was not an option in previous MODFLOW versions as the designation was by layer. The NPF Package also has several options for simulating drainage problems and problems involving perched aquifers where an active cell overlies a partially saturated cell. The default NPF Package behavior (in which none of these options are set) is the most stable for typical groundwater problems. The default NPF Package behavior does not correspond to the default behavior for other MODFLOW internal flow packages. The NPF Package does not support quasi-3D confining units. The NPF Package replaces the Layer Property Flow (LPF), Block-Centered Flow (BCF), and Upstream Weighting (UPW) Packages from previous MODFLOW versions. Capabilities of the Hydrogeologic Unit Flow (HUF) Package (Anderman and Hill, 2000, 2003) are not supported in the GWF Model of MODFLOW 6 .

8. Aquifer storage properties are specified in the Storage (STO) Package. If the STO Package is excluded for a model, then the model represents steady-state conditions. If the STO Package is included, users can specify steady-state or transient conditions by stress period as needed. Compressible storage contributions are no longer approximated as zero for unconfined layers; contributions from pore drainage and compressible storage are separated in the model output. 
9. The Horizontal Flow Barrier (HFB) Package (Hsieh and Freckleton, 1993; Harbaugh, 2005) in MODFLOW 6 allows barrier properties and locations to change by stress period. The capability to change barrier properties by stress period was not supported in previous MODFLOW versions.

10. The GWF Model in MODFLOW 6 allows multiple stress packages of the same type to be specified for a single GWF Model. This capability is also available in MODFLOW-CDSS (Banta, 2011). Package entries written to the budget file and budget terms in the listing file are written separately for each package.

11. Input of boundary conditions for simulation in multiple stress periods is entered differently than for previous MODFLOW versions. Boundary conditions are specified for a stress period in a "PERIOD" block. These boundary conditions remain active at their specified values until a subsequent "PERIOD" block is encountered or the end of the simulation is reached. Individual entries within the "PERIOD" block can be specified as a time-series entry. Values for these variables, which may correspond to a well pumping rate or a drain conductance, for example, are interpolated from a time-series dataset, for each time step, using several different interpolation options.

12. The Flow and Head Boundary (FHB) Package (Leake and Lilly, 1997; Harbaugh, 2005) is not supported in MODFLOW 6 ; however, its capabilities can be replicated using the WEL Package, the CHD Package, and the new time-series capability.

13. There is one Evapotranspiration (EVT) Package for MODFLOW 6 . The MODFLOW 6 EVT Package contains the functionality of the MODFLOW-2005 EVT Package, the Segmented Evapotranspiration (ETS) Package (Banta, 2000), and the Riparian Evapotranspiration (RIP-ET) Package (Maddock and others, 2012).

14. A new Multi-Aquifer Well (MAW) Package replaces the Multi-Node Well (MNW1 and MNW2) Packages (Halford and Hanson, 2002; Konikow and others, 2009). The new package does not contain all of the options available in MNW1 and MNW2, but it does contain the most commonly used ones. It also has new capabilities for simulating flowing wells. The MAW Package is solved as part of the matrix solution and is tightly coupled with the GWF Model. This tight coupling with the GWF Model may substantially improve convergence for simulations of groundwater flow to multi-aquifer wells.

15. Most capabilities of the Stream (STR) and Streamflow Routing (SFR) Packages (Prudic, 1989; Prudic and others, 2004; Niswonger and Prudic, 2005) are included in MODFLOW 6 as a new SFR Package. The SFR Package documented in this report contains all of the functionality of the SFR Package in MODFLOW-2005 with the following exceptions: (a) the concept of a "segment" has been eliminated, (b) only rectangular cross sections are supported for stream reaches, and (c) unsaturated zone flow beneath stream reaches cannot be simulated.

16. A new Lake (LAK) Package replaces the existing MODFLOW Lake Packages (Merritt and Konikow, 2000). In addition to being able to represent lakes that are incised into the model grid, the new LAK Package can also represent sub-grid scale lakes that are conceptualized as being on top of the model. The new package contains most of the capabilities available in previous LAK Packages. The LAK Package documented here does not represent unsaturated zone flow beneath a lake or support for the coalescing lake option described in Merritt and Konikow (2000).

17. A new Unsaturated Zone Flow (UZF) Package, based on the one described by Niswonger and others (2006), is included in the GWF Model of MODFLOW 6. The new UZF Package allows the UZF capabilities to be applied to only selected cells of the GWF model. The new UZF Package also supports a multi-layer option, which allows for vertical heterogeneity in unsaturated zone properties. 


\section{1-10 Documentation for the MODFLOW 6 Groundwater Flow Model}

18. A new Water Mover (MVR) Package is included in MODFLOW 6. The MVR Package can be used to transfer water from individual "provider" features of selected packages (WEL, DRN, RIV, GHB, MAW, SFR, LAK, and UZF) to individual "receiver" features of the advanced packages (MAW, SFR, LAK, and UZF). Simple rules are used to determine how much of the available water is moved from the provider to the receiver, which allows management controls to be represented.

19. MODFLOW 6 contains a flexible new Observation (OBS) capability, which allows the user to define many different types of continuous-in-time or point-in-time observations. The new OBS capability replaces the Observation Process (Hill and others, 2000), the Gage Package, and the HYDMOD capability (Hanson and Leake, 1999) in previous MODFLOW versions. Flow, head, and drawdown observations can be obtained for the GWF Model. Flow and other package-specific observations, such as the head in a multi-aquifer well or lake stage, for example, can also be obtained. These observed values can be used subsequently with a parameter estimation program or they can be used to make time-series plots of a wide range of simulated values. The new OBS capability does not support specification of field-measured observations, calculation of residuals, or interpolation within a grid, as was supported in previous versions of the MODFLOW OBS Process.

20. The GWF Model described in this report does not support the following list of packages and capabilities. Support for some of these capabilities may be added in future MODFLOW 6 versions.

- Interbed Storage Package (Leake and Prudic, 1991),

- Subsidence Package (Hoffmann and others, 2003),

- Subsidence and Aquifer-System Compaction Package for Water-Table Aquifers (Leake and Galloway, 2007),

- Drain with Return Flow Package (Banta, 2000)

- Reservoir Package (Fenske and others, 1996),

- Seawater Intrusion Package (Bakker and others, 2013),

- Surface-Water Routing Process (Hughes and others, 2012),

- Connected Linear Network Process (Panday and others, 2013),

- Parameter Value File (Harbaugh, 2005), and

- Link to the MT3DMS Contaminant Transport Model (Zheng and others, 2001).

21. There are other differences between MODFLOW 6 and previous MODFLOW versions regarding input and output. Descriptions for the MODFLOW 6 input and output are described in a separate user guide, which is included with the distribution. One of the most visible changes to the input files is the use of keywords to label the individual input variables. This improvement makes the input files human readable, reduces the likelihood of input errors, and makes it easier to support backward compatibility as new capabilities are added to the program.

Successful application of the GWF Model in MODFLOW 6 requires users to make many fundamental decisions about spatial and temporal discretization, ways to represent boundary conditions, and so forth. For many MODFLOW users, particularly those unfamiliar with the newer MODFLOW variants, there are two new fundamental decisions to make. The first is deciding which type of grid to use. Regular MODFLOW grids consisting of layers, rows, and columns are appealing for many reasons and are recommended for most applications. It is easy to prepare input data for regular MODFLOW grids, contouring of model results is straightforward, and there are many preprocessing and postprocessing tools available. On the other hand, the flexibility offered by the DISV and DISU Packages is also appealing, especially when there is a need to focus resolution around wells, streams, or other areas of interest. There are special geometric requirements for cells and 
their connections that users must consider when designing unstructured grids, and preprocessing and postprocessing capabilities in support of these requirements may be limited or unavailable. Over time it is expected that the tools for designing the various types of unstructured grids (nested, quadtree, Voronoi, and different structures by layer) and for postprocessing the results (contouring, particle tracking, budget calculations) will become widely available; therefore, this powerful approach to MODFLOW modeling will become more widely adopted. Determining the formulation to use (the standard formulation or the Newton-Raphson formulation) is the second fundamental decision that users must make for each application. The standard formulation should be used for confined aquifers and for models of unconfined aquifers where cell wetting and drying is not problematic. For all other applications, the Newton-Raphson formulation is recommended. The NewtonRaphson formulation provides robust solutions for many complex water-table problems that may not converge with the standard formulation. Those familiar with previous MODFLOW documentation will note that this report is largely an extension of Harbaugh (2005) to include unstructured grid capabilities and the NewtonRaphson formulation, as well as to present the advanced packages, the capability to tightly couple multiple GWF Models at the matrix level, and other recent MODFLOW advances.

\section{Organization and Scope of This Report}

MODFLOW 6 is being released initially with only the GWF Model. Because the object-oriented framework is new in MODFLOW 6, a general description of the framework is provided as an appendix. This report documenting MODFLOW 6 is similar to Harbaugh (2005) in that it describes the fundamental concepts for the GWF Model; however, the programmer information and user input instructions are not included in this report, as was done in Harbaugh (2005). Instead, user input instructions are provided as a separate document with the program distribution. By distributing the input instructions separately, they can evolve with the program as new options are added. In some instances, users may need to refer to additional reports for capabilities added to MODFLOW, including additions of processes and capabilities to simulate additional hydrologic features.

The purpose of this report is to describe the mathematical concepts used in the GWF Model of MODFLOW 6. Chapter 2 derives the control-volume finite-difference (CVFD) equation upon which the GWF Model is based. The core parts of the GWF Model are described in chapters 3, 4, and 5. Chapter 3 describes the discretization packages, of which there are three choices. Chapter 4 describes the internal flow packages, which consist of the NPF, HFB, and GNC Packages. Chapter 5 describes the STO Package. Chapter 6 describes the conceptualization and implementation of stress packages. The description of each stress consists of the physical and mathematical concepts and the derivation of the terms that incorporate the stress into the flow equation. For example, in the section on the River Package, an equation is derived that approximates flow through a riverbed, and a discussion is provided to show how that equation can be incorporated into the finite-difference equation. Chapter 7 describes the advanced stress packages included with the GWF Model in MODFLOW 6. These include the SFR, LAK, MAW, UZF, and MVR Packages. Chapter 8 describes the GWFGWF Exchange for hydraulically connecting two GWF Models. Appendix A contains a list of mathematical symbols used in this report. Appendix B describes the implementation of the GWF Model in the MODFLOW 6 framework.

The GWF Model packages documented in this report are as follows:

- Spatial Discretization

- Initial Conditions

- Output Control

- Node Property Flow

- Horizontal Flow Barrier 


\section{1-12 Documentation for the MODFLOW 6 Groundwater Flow Model}

- Storage

- Ghost Node Correction

- Time-Variant Specified Head

- Well

- Recharge

- General-Head Boundary

- River

- Drain

- Evapotranspiration

- Streamflow Routing

- Lake

- Multi-Aquifer Well

- Unsaturated Zone Flow

- Water Mover

The following framework components are described in a separate MODFLOW 6 framework report (Hughes and others, 2017).

- Simulation (Main Program)

- Timing Module

- Solution Object

- Model Object

- Exchange Object

- Time Series

- Observations 


\section{Chapter 2. Formulation and Solution of the Control-Volume Finite- Difference Equation}

The groundwater flow equation in MODFLOW 6 is discretized using a control-volume finite-difference (CVFD) method. This chapter describes the mathematical equations discretized in the groundwater flow model, discretization options, the general isotropic and anisotropic forms of the finite-difference equations used to simulate horizontal and vertical groundwater flow, and finite-difference equations for head-dependent and specified boundary condition flows. In MODFLOW-2005, finite-difference equations are formulated in terms of intercell conductance terms, boundary condition conductances, and specified flows. In this report, this traditional MODFLOW formulation is referred to as the "standard formulation."

The Newton-Raphson method is another widely used method for solving unconfined groundwater flow problems that has been shown to be a useful alternative to the standard formulation for many problems (Painter and others, 2008; Keating and Zyvoloski, 2009). In general, the Newton-Raphson method can improve solution convergence and avoid problems with the drying and rewetting of cells. Newton-Raphson equations (referred to as the "Newton-Raphson formulation" in this document) that can be used to simulate unconfined groundwater flow are also presented along with the general solution procedures used to solve the discretized equations.

In large part, the numerical approaches used in MODFLOW 6 are based on MODFLOW-NWT (Niswonger and others, 2011), MODFLOW-USG (Panday and others, 2013), and MODFLOW-2005 (Harbaugh, 2005). The numerical approaches also draw from the concepts summarized in popular groundwater modeling text books (Remson and others, 1971; Bear, 1979; Wang and Anderson, 1982; Anderson and Woessner, 1992; Anderson and others, 2015).

\section{Mathematical Model}

The three-dimensional movement of groundwater of constant density through porous earth material is described by Darcy's Law:

$$
\mathbf{q}=-\mathbf{K} \nabla h=-\left(\begin{array}{ccc}
K_{x x} & 0 & 0 \\
0 & K_{y y} & 0 \\
0 & 0 & K_{z z}
\end{array}\right) \nabla h
$$

where $\mathbf{q}$ is a vector of specific discharge (L/T), or fluid-flux vector, $\mathbf{K}$ is the hydraulic-conductivity tensor (L/T); $K_{x x}, K_{y y}$, and $K_{z z}$ are values of hydraulic conductivity along the $x, y$, and $z$ coordinate axes, which are assumed to be parallel to the major axes of hydraulic conductivity (L/T); $h$ is the potentiometric head (L); and $\nabla h$ is the head-gradient vector. When combined with a water balance on a small control volume, Darcy's Law leads to a partial-differential equation that describes the distribution of hydraulic head:

$$
\frac{\partial}{\partial x}\left(K_{x x} \frac{\partial h}{\partial x}\right)+\frac{\partial}{\partial y}\left(K_{y y} \frac{\partial h}{\partial y}\right)+\frac{\partial}{\partial z}\left(K_{z z} \frac{\partial h}{\partial z}\right)+Q_{s}^{\prime}=S S \frac{\partial h}{\partial t}
$$

where $Q_{s}^{\prime}$ is a volumetric flux per unit volume representing sources and sinks of water, with $Q_{s}^{\prime}$ being negative for flow out of the groundwater system, and $Q_{s}^{\prime}$ being positive for flow into the system $\left(\mathrm{T}^{-1}\right) ; S S$ is the specific storage of the porous material $\left(\mathrm{L}^{-1}\right)$; and $t$ is time $(\mathrm{T})$.

For an example of a derivation of equation 2-2 see for example Rushton and Redshaw (1979). In general, $S S, K_{x x}, K_{y y}$, and $K_{z z}$ may be functions of space $\left(S S=S S(x, y, z), K_{x x}=K_{x x}(x, y, z)\right.$, and so forth) and 


\section{2-2 Documentation for the MODFLOW 6 Groundwater Flow Model}

$Q_{s}^{\prime}$ may be a function of space and time $\left(Q_{s}^{\prime}=Q_{s}^{\prime}(x, y, z, t)\right)$. Equation 2-2 describes transient groundwater flow in a heterogeneous and anisotropic medium, provided the principal axes of hydraulic conductivity are aligned with the coordinate directions. Equation 2-2, together with specification of flow and head conditions at the boundaries of an aquifer system and specification of initial-head conditions, constitutes a mathematical representation of a groundwater flow system. A solution of equation 2-2, in an analytical sense, is an algebraic expression giving $h(x, y, z, t)$ such that, when the derivatives of $h$ with respect to space and time are substituted into equation $2-2$, the equation and its initial and boundary conditions are satisfied. A time-varying head distribution characterizes the flow system, in that it measures both the energy of flow and the volume of water in storage; it also can be used to calculate directions and rates of movement.

\section{Control-Volume Finite-Difference Method}

Except for very simple systems, analytical solution of equation 2-2 is rarely possible, so various numerical methods must be employed to obtain approximate solutions. One such approach is the CVFD method, wherein the continuous system described by equation 2-2 is replaced by a finite set of discrete points in space and time, and the partial derivatives are replaced by terms calculated from the differences in head values at these points. The process leads to a system of simultaneous linear algebraic difference equations; the solution yields values of head at specific points and times. These values constitute an approximation to the time-varying head distribution that would be given by an analytical solution of the partial-differential equation of flow.

The CVFD analog of equation 2-2 may be derived by applying the rules of difference calculus; however, in the discussion presented here, an alternative approach is used with the aim of simplifying the mathematical treatment and explaining the computational procedure in terms of familiar physical concepts regarding the flow system.

In MODFLOW 6, the discrete control volumes that comprise the model domain are called model "cells." Within each cell there is a point called a "node" at which head is to be calculated. Many schemes for locating nodes in cells could be used; however, the finite-difference equation developed in the following section uses the block-centered formulation in which the nodes are at the center of the cells. Hydraulic communication between cells is conceptualized in terms of conductive connections between nodes. The assemblage of model cells and its associated network of connections is called the model "grid."

\section{Structure of a Model Cell}

A MODFLOW 6 model cell is a prism with vertical sides and a horizontal top and bottom (fig. 2-1). In previous MODFLOW versions (with the exception of MODFLOW-USG), the outline of a cell, as viewed from the top, was rectangular. In MODFLOW 6, the outline of a cell may be any convex polygon, including a rectangular polygon. A convex polygon is defined as one in which all interior angles are less than 180 degrees. Points at which the edges of a polygon meet are called "vertices." Because the sides of a cell are vertical, the same polygon defines the shape of the top and bottom of the cell. The area of the polygon is called the "horizontal area" of the cell. The volume of a cell can be calculated as the product of the horizontal cell area and the difference between the cell top and cell bottom elevations.

\section{Connections Between Cells}

A connection between the nodes of two cells indicates that the cells are in hydraulic communication where groundwater can flow between the cells under the influence of a hydraulic gradient. A connection can be either "horizontal" or "vertical" (fig. 2-2). Cell connections are either calculated by MODFLOW 6 using information about the grid, or provided by the user as part of the model input. Likewise, the type of connection (horizontal or vertical) is determined from the type of grid or is specified by the user as input. 
$\boldsymbol{A}$

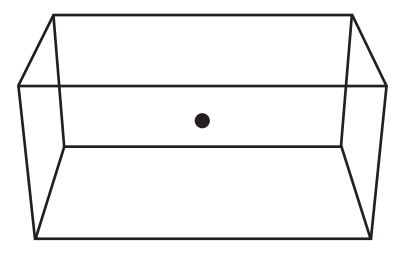

C

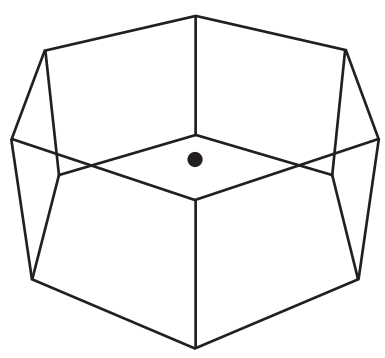

B

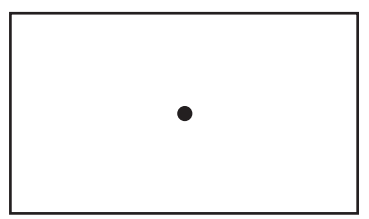

D

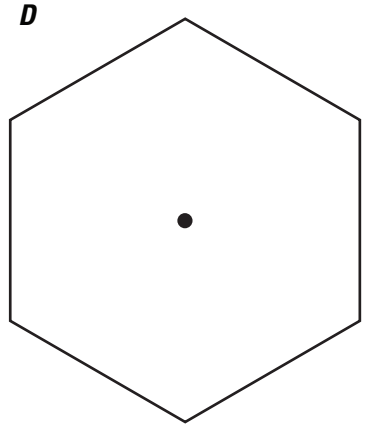

Figure 2-1. Diagram showing MODFLOW 6 model cells with nodes located at their centers. $A$, rectangular cell, oblique view; $B$, outline of rectangular cell as viewed from above; $C$, hexagonal cell, oblique view; $D$, outline of hexagonal cell as viewed from above.

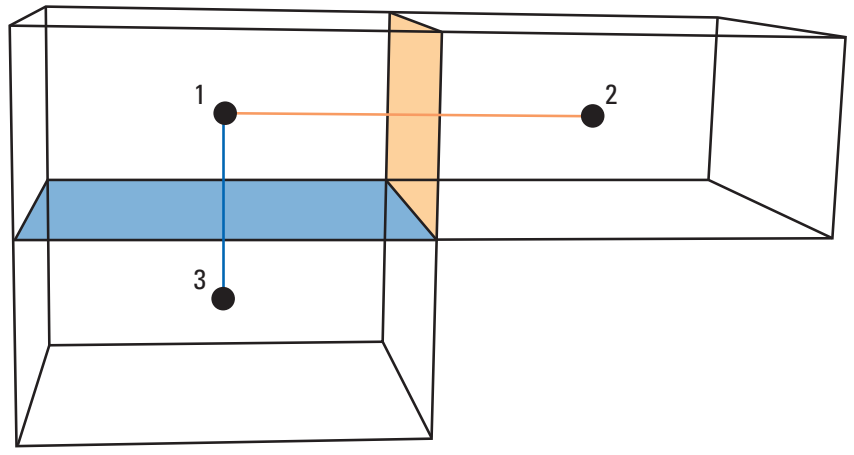

Figure 2-2. Diagram showing connections between the nodes of three model cells. Cells 1 and 2 have a horizontal connection that crosses their shared vertical side (green). Cells 1 and 3 have a vertical connection that crosses their shared horizontal bottom/top (blue). 


\section{2-4 Documentation for the MODFLOW 6 Groundwater Flow Model}
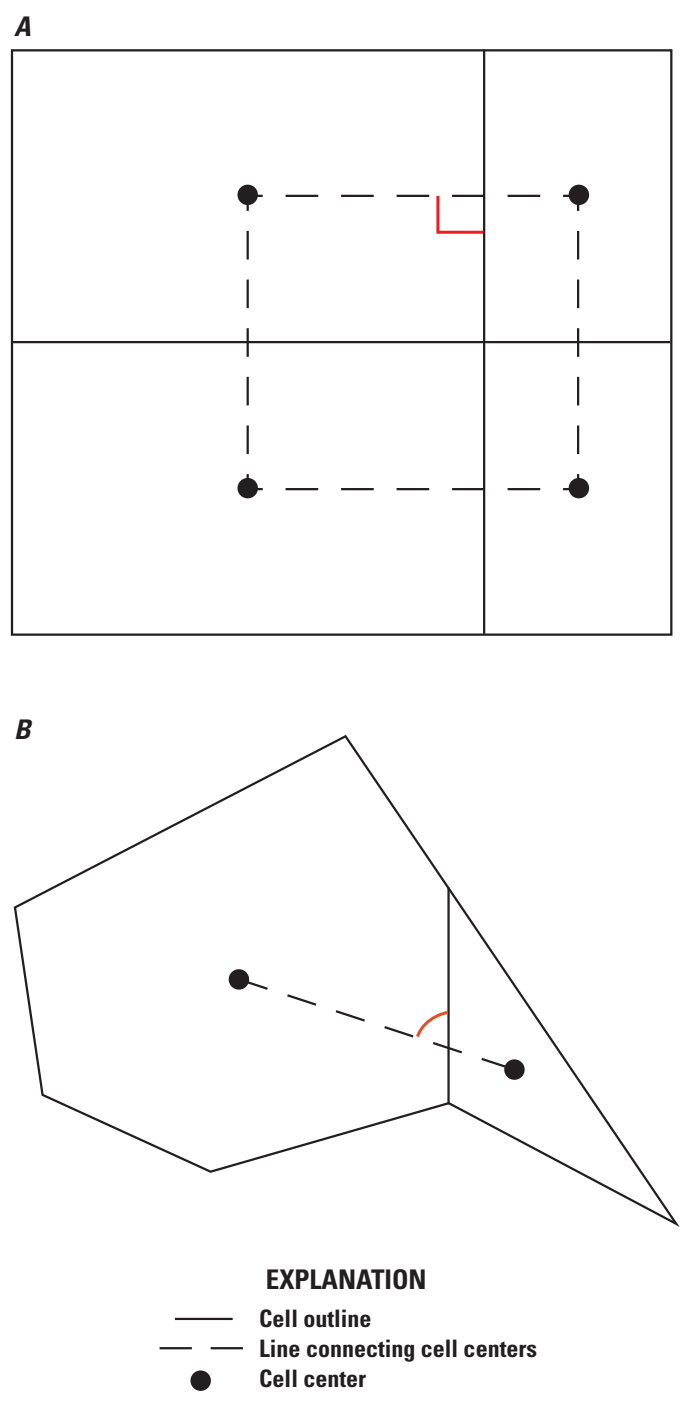

Figure 2-3. Diagram showing examples of two different types of cell connections. $A$, a line connecting the centers of adjacent cells passes through the shared face at a right angle; and $B$, a connecting line does not intersect the shared face at a right angle. Figure modified from Panday and others (2013).

Horizontal connections are often horizontal in a strict geometric sense; that is, they often lie within the $(x, y)$ coordinate plane, as appears to be the case in figure 2-2. Similarly, vertical connections are often strictly vertical; that is, they often are aligned with the $z$ coordinate direction. In general, however, the terms "horizontal" and "vertical," as applied to a connection, do not necessarily imply strict horizontal or vertical orientation of the connection. Rather, they (indirectly) indicate the type and orientation of the interface with which a connection is associated. A horizontal connection crosses a vertical interface formed by cell sides, and a vertical connection crosses a horizontal interface formed by a cell top and a cell bottom.

For accurate solutions with the CVFD method, there are geometric requirements regarding cell connections. First, a line drawn between the centers of any two connected cells should intersect the shared face at a right angle (fig. 2-3). Second, the intersection point should coincide with an appropriate mean position on the shared face (Narasimhan and Witherspoon, 1976). The smaller the deviation from these CVFD connection requirements, the smaller the loss in accuracy in the groundwater flow solution. 


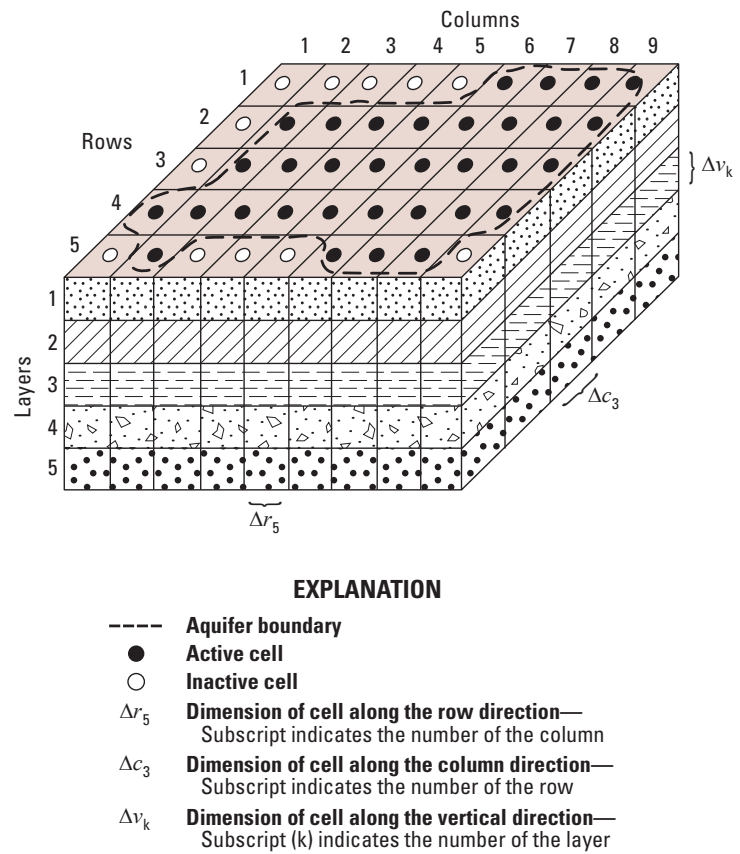

Figure 2-4. Illustration showing a hypothetical aquifer system discretized using a regular MODFLOW grid. Figure modified from Harbaugh (2005).

\section{Model Grids}

The collection of all model cells and their connections is called the model "grid." With the exception of MODFLOW-USG, previous versions of MODFLOW supported only "regular" grids composed of rows, columns, and layers of rectangular-prismatic cells. In addition to regular MODFLOW grids, MODFLOW 6 also supports "unstructured" grids whose cells need not be rectangular and whose connectivity is not restricted to rows, columns, or layers. Unstructured connectivity offers the user considerable flexibility in constructing grids that conform to model domains with irregular geometry and complex geology.

\section{Regular MODFLOW Grids}

Figure 2-4 shows spatial discretization of an aquifer system using a regular MODFLOW grid. A regular MODFLOW grid is a rectilinear grid of cells, the locations of which are described in terms of rows, columns, and layers. A layer, row, and column indexing system is used. For example, figure 2-4 shows a system with five rows, nine columns, and five layers. In formulating the equations of the model, an assumption was made that layers would generally correspond to horizontal geohydrologic units or intervals. Thus, in terms of Cartesian coordinates, the layer index denotes changes along the vertical, $z$. As the convention followed in this model is to number layers from the top down, an increment in the layer index corresponds to a decrease in elevation. Similarly, rows would be considered parallel to the $x$ axis, so that increments in the row index would correspond to decreases in $y$; and columns would be considered parallel to the $y$ axis, so that increments in the column index would correspond to increases in $x$. These conventions were followed in constructing figure 2-4; however, application of the model requires only that rows and columns fall along consistent orthogonal directions within the layers, and does not require the designation of $x, y$, or $z$ coordinate axes.

In three dimensions, discretization of an aquifer system with a regular MODFLOW grid results in a sevenpoint structured connectivity for the discretized set of equations. This means that a single model cell is con- 


\section{2-6 Documentation for the MODFLOW 6 Groundwater Flow Model}

nected, at most, to six surrounding model cells. Because the number of connections remains constant in space (except along boundaries), a regular MODFLOW grid is also considered a structured grid, because the number of connections is the same for all cells.

\section{Generalized Structured and Unstructured Grids}

The term "unstructured grid" simply means that the number of connections may be variable for each cell within a grid. This variability results in an unstructured system of equations. In the CVFD method, the connectivity of a cell depends on the number of shared faces, which may vary for each cell. A structured grid, in which the number of shared faces is constant throughout a grid, is considered a subset of unstructured grids.

Figure 2-5 shows examples of different types of structured and unstructured grids that may be defined for the GWF Process in MODFLOW 6. The top part of figure 2-5 shows structured model grids in which the number of connections is the same for all cells (except for along boundaries). For structured rectangular grids (fig. 2-5A), the CVFD methodology is identical to a conventional or standard finite-difference approximation (for example, Peaceman 1977, and Moridis and Pruess 1992). Simple structured grids consisting of hexagons, equilateral triangles, and rectangles meet the CVFD connection requirements. The triangular and quadrilateral grids shown in figure $2-5 F$ and $G$ are structured, but the grids have been warped to fit a domain outline. Warping a grid can be an effective way to efficiently discretize an irregular domain, but by warping the grid, the CVFD connection requirements are often violated.

For the unstructured grids shown in the bottom part of figure 2-5, the number of connections for each cell is variable throughout the grid. The capability of unstructured grids to support local increases in resolution can be useful and efficient when the scale of interest or the magnitude of the hydraulic gradient varies throughout the domain. With the exception of the radial grid (fig. 2-5J), the unstructured grids in figure 2-5 do not meet the CVFD connection requirements.

\section{Flows Between Cells (Internal Flows)}

Flows between cells are called "internal flows." Mathematical expressions for internal flows are discrete forms of Darcy's Law. Under certain conditions, which are discussed later in this chapter, the flow between two cells can be expressed accurately as the product of a hydraulic conductance and the head difference between the two cells. This is called the "conductance-based" flow expression.

To simplify the following development, flows are considered positive if they are entering cell $n$; the negative sign usually incorporated in Darcy's Law has been dropped from all terms. Following these conventions, horizontal flow into cell $n$ from cell $m$ (fig. 2-6) is given by Darcy's Law as

$$
Q_{n, m}=\bar{K}_{n, m} \Delta w_{n, m} \Delta v_{n, m} \frac{h_{m}-h_{n}}{L_{n, m}+L_{m, n}}
$$

where $Q_{n, m}$ is the flow rate into cell $n$ from cell $m, \bar{K}_{n, m}$ is the effective hydraulic conductivity between the nodes (details for calculating $\bar{K}_{n, m}$ are described in chapter 4), $\Delta w_{n, m}$ is the width of the face through which flow occurs, $\Delta v_{n, m}$ is the height of the face through which flow occurs, $h_{m}$ is the head at node $m, h_{n}$ is the head at node $n, L_{n, m}$ is the distance from the center of cell $n$ to its shared face with cell $m$, and $L_{m, n}$ is the distance from the center of cell $m$ to its shared face with cell $n$.

Equation 2-3 gives the exact flow for a one-dimensional steady-state case through a block of aquifer extending from node $m$ to node $n$ and having a cross-sectional area $\Delta w_{n, m} \Delta v_{n, m} . \bar{K}_{n, m}$ is the conductivity of the material between nodes $n$ and $m$, which is the effective hydraulic conductivity for the entire region between the nodes, normally calculated as a harmonic mean, as described by Collins (1961), for example. Similar expressions can be written approximating the flow into cell $n$ from all its neighboring $m$ cells. 
Structured grids

A. Rectangular grid

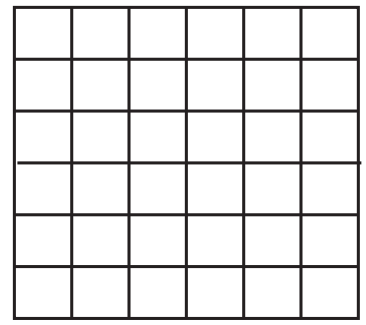

E. Hexagonal grid

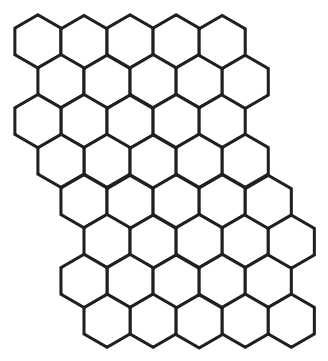

Unstructured grids

$H$. Rectangular, nested grid

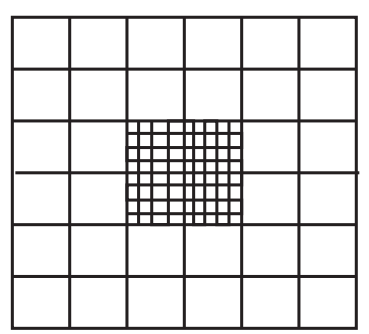

$K$. Rectangular, quadtree grid, no smoothing

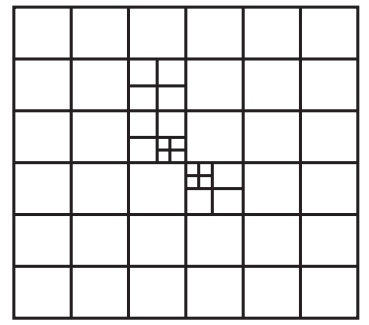

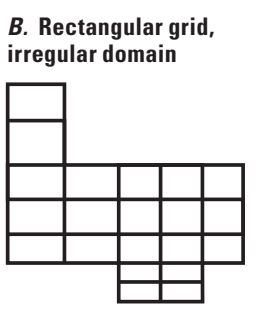

C. Triangular grid, isosceles triangles

D. Triangular grid, equilateral

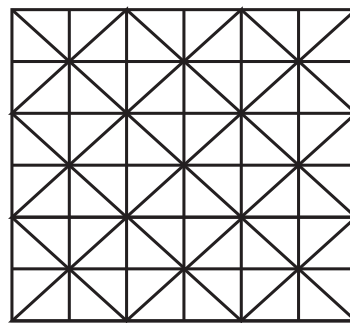
triangles
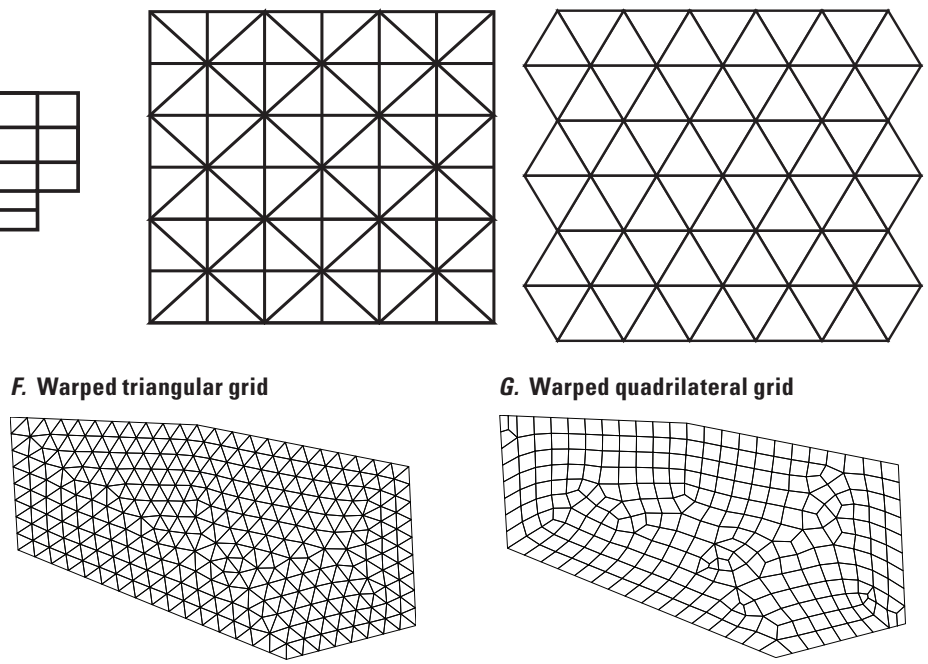

G. Warped quadrilateral grid

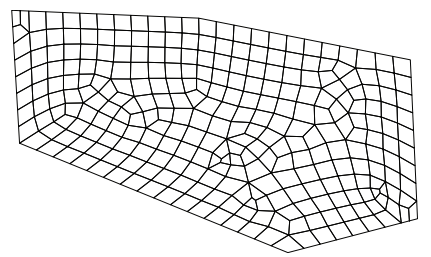

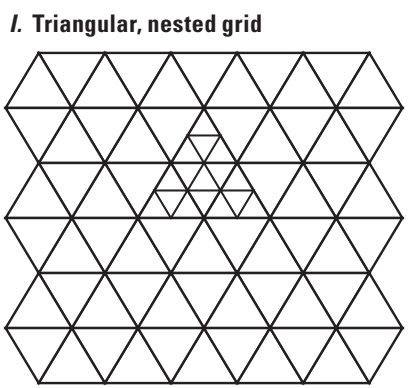

L. Rectangular, quadtree grid, with smoothing

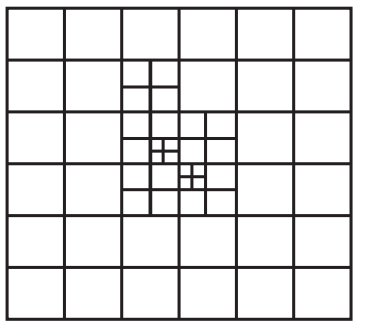

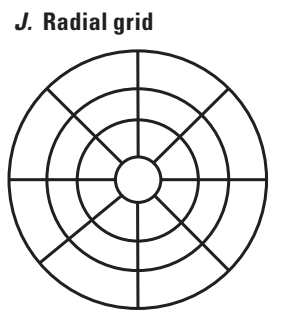

M. Irregular polygon grid

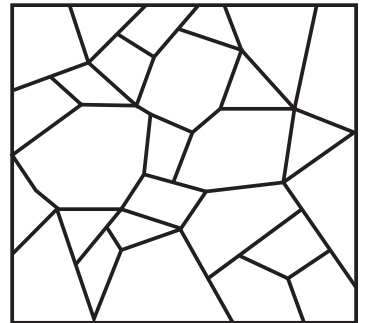

Figure 2-5. Illustration showing examples of different types of structured and unstructured grids. Figure modified from Panday and others (2013). 


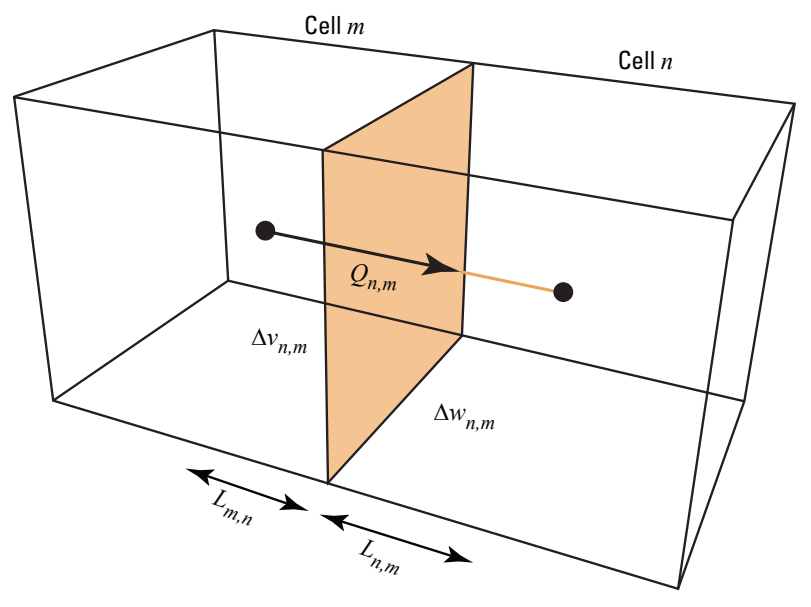

Figure 2-6. Diagram showing flow into cell $n$ from cell $m$. Figure modified from Harbaugh (2005).

Equation 2-3 expresses inflow through a face of cell $n$ in terms of heads, grid dimensions, and hydraulic conductivity. This notation can be simplified by combining grid dimensions and hydraulic conductivity into a single constant, the "hydraulic conductance" or, more simply, the "conductance." For example,

$$
C_{n, m}=\frac{\bar{K}_{n, m} \Delta w_{n, m} \Delta v_{n, m}}{L_{n, m}+L_{m, n}}
$$

where $C_{n, m}$ is the conductance between nodes $n$ and $m\left(\mathrm{~L}^{2} \mathrm{~T}^{-1}\right)$. Thus, conductance is the product of hydraulic conductivity and cross-sectional area of flow divided by the length of the flow path (in this case, the distance between the nodes). Chapter 4 describes the calculation of conductance for horizontal and vertical flow for both confined and partially saturated conditions.

Substituting conductance from equation 2-4 into equation 2-3 yields the conductance-based flow expression,

$$
Q_{n, m}=C_{n, m}\left(h_{m}-h_{n}\right)
$$

\section{External Sources and Sinks (External Flows)}

Equation 2-5, when written for all $m$ cells connected to cell $n$, accounts for the flow from adjacent cells. To account for flows into the cell from features or processes external to the aquifer, such as rivers, drains, areal recharge, evapotranspiration, or wells, additional terms are required. These external flows may be dependent on the head in the receiving cell but independent of all other heads in the aquifer, or they may be entirely independent of the head in the receiving cell. Flow from outside the aquifer may be represented by the expression

$$
a_{n, i s r c}=p_{n, i s r c} h_{n}+q_{n, i s r c}
$$

where $a_{n, i s r c}$ represents flow from external source $i s r c$ into cell $n\left(\mathrm{~L}^{3} \mathrm{~T}^{-1}\right), p_{n, i s r c}$ is the head coefficient used in the flow calculation $\left(\mathrm{L}^{2} \mathrm{~T}^{-1}\right)$, and $q_{n, i s r c}$ is the head independent term used in the flow calculation $\left(\mathrm{L}^{3} \mathrm{~T}^{-1}\right)$. 


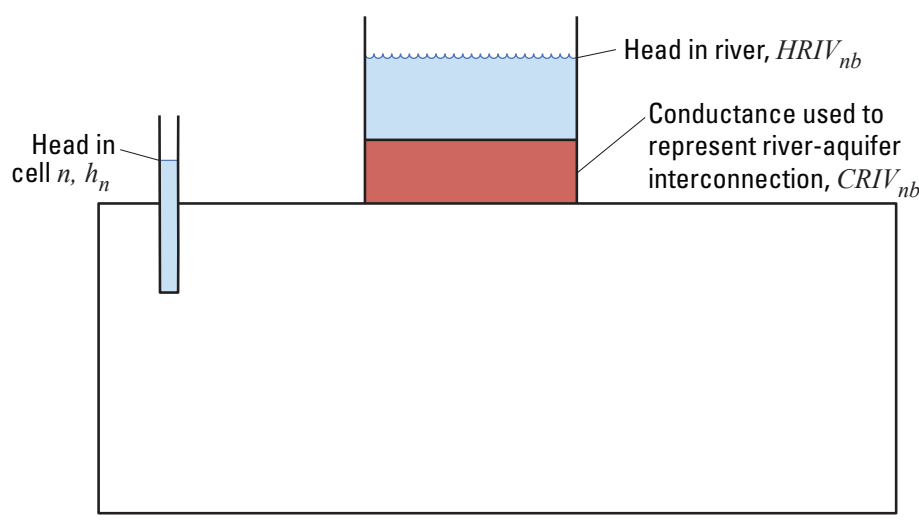

Figure 2-7. Diagram showing conceptual representation of leakage through a riverbed into a model cell. Figure modified from Harbaugh (2005).

For example, suppose a cell is receiving flow from two sources-recharge from a well and seepage through a riverbed. For the first source ( $i \operatorname{src}=1$ ), because the flow from the well is assumed to be independent of head, $p_{n, 1}$ is zero and $q_{n, 1}$ is the injection rate for the well. In this case,

$$
a_{n, 1}=q_{n, 1}
$$

For the second source $(i \operatorname{src}=2$ ), the assumption is made that the river-aquifer interconnection can be treated as a simple conductance so that the seepage is proportional to the head difference between the river stage and the head in cell $n$ (fig. 2-7); thus, we have

$$
a_{n, 2}=C R I V_{n, 2}\left(R_{n, 2}-h_{n}\right),
$$

where $C R I V_{n, 2}$ is a conductance $\left(\mathrm{L}^{2} \mathrm{~T}^{-1}\right)$ controlling flow from the river into cell $n$ and $R_{n, 2}$ is the head in the river $(\mathrm{L})$.

For example, in the situation shown schematically in figure 2-7, $C R I V$ would be given as the product of the vertical hydraulic conductivity of the riverbed material and the area of the riverbed as it crosses the cell, divided by the thickness of the riverbed material. Equation $2-8$ can be rewritten as

$$
a_{n, 2}=-C R I V_{n, 2} h_{n}+C R I V_{n, 2} R_{n, 2} .
$$

The negative conductance term, $-C R I V_{n, 2}$, corresponds to $p_{n, 2}$ of equation 2-6, while the term $C R I V_{n, 2} R_{n, 2}$ corresponds to $q_{n, 2}$. Similarly, all other external sources or stresses can be represented by an expression of the form of equation 2-6. In general, if there are $n s r c$ external sources or stresses affecting a single cell, the combined flow is expressed by

$$
\sum_{i s r c=1}^{n s r c} a_{n, i s r c}=\sum_{i s r c=1}^{n s r c}\left(p_{n, i s r c} h_{n}\right)+\sum_{i s r c=1}^{n s r c} q_{n, i s r c} .
$$




\section{2-10 Documentation for the MODFLOW 6 Groundwater Flow Model}

Defining $P_{n}$ and $Q_{n}$ by the expressions

$$
P_{n}=\sum_{i s r c=1}^{n s r c} p_{n, i s r c}
$$

and

$$
Q_{n}=\sum_{i s r c=1}^{n s r c} q_{n, i s r c}
$$

the general external flow term for cell $n$ is

$$
Q_{n, s}=\sum_{i s r c=1}^{n s r c} a_{n, i s r c}=P_{n} h_{n}+Q_{n} .
$$

Depending on the type of external flow representation, there are some cases where $p_{n, i s r c}$ or $q_{n, i s r c}$ (or both) may be a function of the head in the cell $\left(h_{n}\right)$. In these situations, values for $p_{n, i s r c}$ and $q_{n, i s r c}$ are typically calculated using a previous estimate of $h_{n}$.

\section{Derivation of Control-Volume Finite-Difference Equations}

Equation 2-2, the governing equation for three-dimensional groundwater flow, represents a water balance on a small control volume within the model domain. The terms involving components of hydraulic conductivity represent rates of flow into or out of the control volume under the influence of the hydraulic gradient. The term $Q_{s}^{\prime}$ represents the rate at which water enters or leaves the control volume via other sources and sinks. The term involving specific storage, $S S$, represents the rate at which the water stored in the control volume is accumulated or depleted.

The CVFD equations solved by MODFLOW 6 are discrete analogs of equation 2-2 in which differential control volumes are approximated by model cells of finite volume. Just as equation 2-2 is a water balance on control volumes, the CVFD equations are water balances on model cells.

The derivation of the CVFD equations begins in this chapter with a general water balance on a cell that takes into account groundwater flows between cells (internal flows), flows from and to other sources and sinks (external flows), and storage. Internal flows are calculated using the flow expression (eq. 2-5) developed in the previous section. The resulting CVFD equations are discrete analogs of the governing equation (eq. 2-2). Details of how conductances are calculated from the hydraulic properties, geometry of the model grid, and cell saturation are discussed in chapter 4.

\section{Water Balance on a Cell}

Development of the groundwater flow equation in CVFD form follows from the application of the continuity equation: The sum of all internal flows into and out of the cell plus the sum of all source and sink flows must be equal to the rate of change in storage within the cell. Under the assumption that the density of groundwater is constant, the continuity equation expressing the balance of flow for a cell $n$ is

$$
\sum_{m \in \eta_{n}} Q_{n, m}+Q_{n, s}-Q_{S T O}=0
$$


where $\eta_{n}$ is a list of the cells connected to cell $n, Q_{n, m}$ is the flow rate into cell $n$ from cell $m\left(Q_{n, m}\right.$ is negative if flow is from cell $n$ to cell $m$ ) with dimensions of $\mathrm{L}^{3} \mathrm{~T}^{-1}, Q_{n, s}$ is the flow rate of sources and sinks into cell $n\left(\mathrm{~L}^{3} \mathrm{~T}^{-1}\right)$, and $Q_{S T O}$ is the change in the volume of water stored in cell $n\left(\mathrm{~L}^{3} \mathrm{~T}^{-1}\right) . Q_{S T O}$ is defined as

$$
Q_{S T O}=S S_{n} \frac{\Delta h_{n}}{\Delta t} V_{n}
$$

where $S S_{n}$ is the volume of water that can be added or removed per unit volume of aquifer material per unit change in head in cell $n\left(\mathrm{~L}^{-1}\right), V$ is the volume of cell $n\left(\mathrm{~L}^{3}\right)$, and $\Delta h$ is the change in head in cell $n$ over a time interval of length $\Delta t$. The definition for $Q_{S T O}$ is expanded in chapter 5 to include specific yield in addition to specific storage.

The term on the right-hand side of equation $2-15$ is equivalent to the volume of water taken into storage or released from storage over a time interval of $\Delta t$ given a change in head of $\Delta h$. Thus, this term is a finite difference approximation for the derivative of head with respect to time. With this sign convention, the $Q_{S T O}$ term is positive when the head in the cell is increasing (water accumulates in the cell). The $Q_{S T O}$ term is negative when the head in the cell is decreasing (water is being released from storage). This convention leads to $Q_{S T O}$ being negative on the left-hand side of equation 2-14. Expanding equation 2-14 using the general external flow term (eq. 2-13) and the storage expression (eq. 2-15) yields

$$
\sum_{m \in \eta_{n}} Q_{n, m}+P_{n} h_{n}+Q_{n}-S S_{n} V_{n} \frac{\Delta h_{n}}{\Delta t}=0 .
$$

For conditions of steady-state groundwater flow, the storage term on the right-hand side of equation 2-16 becomes zero, and the continuity equation reduces to

$$
\sum_{m \in \eta_{n}} Q_{n, m}+P_{n} h_{n}+Q_{n}=0
$$

Under steady-state conditions, internal flows balance with external flows.

\section{Temporal Discretization}

The finite-difference approximation for the time derivative of head, $\Delta h_{n} / \Delta t$, must next be expressed in terms of the head in cell $n$ at two different times. Figure 2-8 shows a hydrograph of head values at node $n$. Two values of time are shown on the horizontal axis: $t$, the time at which the flow terms are evaluated; and $t_{\text {old }}$ a time that precedes $t$. The head values at node $n$ associated with these times are designated by superscript as $h_{n}$ and $H O L D_{n}$, respectively. An approximation to the time derivative of head at time $t$ is obtained by dividing the head difference $h_{n}-H O L D_{n}$ by the time interval $t-t_{\text {old }}$; that is,

$$
\frac{\Delta h_{n}}{\Delta t} \cong \frac{h_{n}-H O L D_{n}}{t-t_{\text {old }}}
$$

Thus, the hydrograph slope, or time derivative, is approximated using the change in head at the node over a time interval that precedes and ends with the time at which flow is evaluated. This is termed a backwarddifference approach, in that $\Delta h / \Delta t$ is approximated over a time interval that extends backward in time from $t$, the time at which the flow terms are calculated, to $t_{\text {old }}$, the previous time the flows were calculated. 


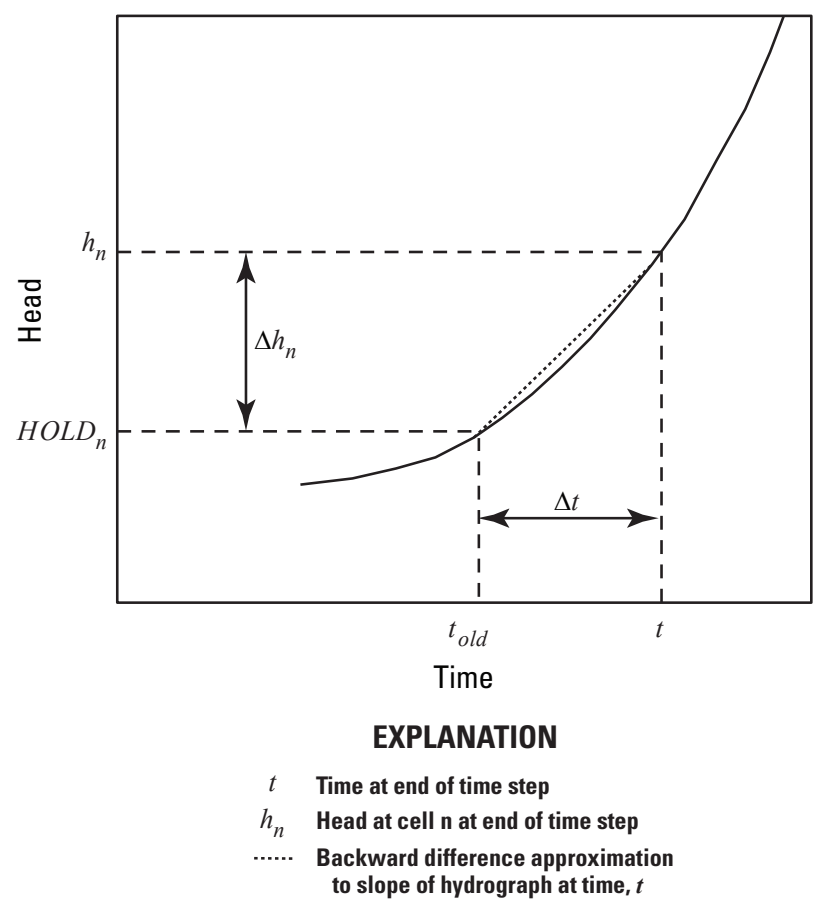

Figure 2-8. Graph of a hydrograph for cell $n$. Figure modified from Harbaugh (2005).

The time derivative of head could be approximated in other ways, for example, we could approximate the time derivative of head over a time interval that begins at the time of flow evaluation and extends to some later time; or over a time interval that is centered at the time of flow evaluation, extending both forward and backward from it. These alternatives, however, may cause numerical instability, resulting in the growth or propagation of error during the calculation of heads at successive times in a simulation. In an unstable situation, errors that enter the calculation for any reason at a particular time will increase at each succeeding time as the calculation progresses, until finally the numerical errors dominate the result. By contrast, the backward-difference approach is always numerically stable - that is, numerical errors do not grow and propagate through time. For this reason, the backward-difference approach is preferred even though is only a first-order approximation and it leads to large systems of equations that must be solved simultaneously for each time step.

\section{Control-Volume Finite-Difference Flow Equation}

The conductance-based flow expression (eq. 2-5) is substituted into the balance equation (eq. 2-16) to give the CVFD flow equation for cell $n$ as

$$
\sum_{m \in \eta_{n}} C_{n, m}\left(h_{m}-h_{n}\right)+P_{n} h_{n}+Q_{n}-S S_{n} V_{n} \frac{\Delta h_{n}}{\Delta t}=0
$$

Equation 2-19 is the generalized CVFD flow equation for cell $n$. This is the traditional finite-difference form of the equation that has been used since MODFLOW was first written. The only difference with previous MODFLOW equations is that the first term on the left-hand side is written in a general form to allow more than six connections. 
Equation 2-19 can be rewritten in backward-difference form by specifying flow terms at $t$, the end of the time interval, and approximating the time derivative of head over the interval $t_{\text {old }}$ to $t$; that is

$$
\sum_{m \in \eta_{n}} C_{n, m}\left(h_{m}-h_{n}\right)+P_{n} h_{n}+Q_{n}-S S_{n} V_{n} \frac{h_{n}-H O L D_{n}}{t-t_{\text {old }}}=0
$$

Equation 2-20 is a backward-difference equation that can be used as the basis for approximating the partialdifferential equation of groundwater flow (eq. 2-2). Like the term $Q_{n}$, the coefficients of the various head terms in equation 2-20 are all known, as is the head at the beginning of the time step, $H O L D_{n}$. The heads at $h_{n}$ and $h_{m}$ at time $t$, the end of the time step, are unknown; that is, they are part of the head distribution to be predicted. Thus, equation 2-20 cannot be solved independently, because it represents a single equation with multiple unknowns. An equation of this type, however, can be written for each active cell in the grid, and, because only one unknown head exists for each cell, we are left with a system of $\mathrm{N}$ equations and $\mathrm{N}$ unknowns. Such a system can be solved simultaneously, subject to appropriate initial and boundary conditions.

\section{Formulation of Standard Equations for Solution}

To prepare the CVFD equation for solution using the standard formulation, it is convenient to rearrange equation 2-20 so that all terms containing heads at the end of the current time step are grouped on the lefthand side of the equation, and all terms that are independent of head at the end of the current time step are on the right-hand side. All coefficients of $h_{n}$ that do not include conductance between nodes or storage are combined into a single term, $H C O F$, and all right-hand-side terms are combined into the term $R H S$. The resulting equation is

$$
\sum_{m \in \eta_{n}} C_{n, m} h_{m}+\left(-\sum_{m \in \eta_{n}} C_{n, m}+H C O F_{n}-\frac{S S_{n} V_{n}}{t-t_{\text {old }}}\right) h_{n}=R H S_{n},
$$

where

$$
H C O F_{n}=P_{n}
$$

and

$$
R H S_{n}=-Q_{n}-S S_{n} V_{n} \frac{H O L D_{n}}{t-t_{\text {old }}}
$$

Because a backward-difference scheme is used, the terms in equations 2-21 to 2-23 are for the end of the time step. For those terms in equations 2-21 to 2-23 that are a function of head $\left(C_{n m}\right.$ for unconfined conditions and $Q_{n}$ and $P_{n}$ for some stress packages, for example), they are calculated using head from a previous iteration.

The entire system of equations of the form of equation 2-21, which includes one equation for each variable- and constant-head cell in the grid, may be written in matrix form as

$$
\mathbf{A h}=\mathbf{b}
$$




\section{2-14 Documentation for the MODFLOW 6 Groundwater Flow Model}

where $\mathbf{A}$ is a matrix of the coefficients of head, from the left side of equation 2-21, for all active and constant head cells in the grid (constant head cells remain in the system of equations by setting their off-diagonal values to zero, their diagonal values to one, and their $R H S$ values to the prescribed head value); $\mathbf{h}$ is a vector of head values at the end of time step, for all active and constant head cells in the grid; and $\mathbf{b}$ is a vector of the constant terms, $R H S$, for all active and constant head cells in the grid. Assembly of the vector $\mathbf{b}$ and the terms that comprise $\mathbf{A}$ occurs through a series of subroutine and method calls by the MODFLOW 6 program. The vector $\mathbf{b}$ and the terms comprising $\mathbf{A}$ are then transferred to the solver, which solves the matrix equations for the vector $\mathbf{h}$. For many groundwater problems, equation 2-24 is nonlinear in that individual entries in the $\mathbf{A}$ matrix are a function of head (the dependent variable). These nonlinearities are resolved through iteration by repeatedly formulating and solving equation 2-24 using A matrix entries recalculated using heads from the previous iteration. Iteration is described in more detail later in this chapter.

\section{Formulation of Newton-Raphson Equations for Solution}

The Newton-Raphson formulation is an alternative to the standard formulation. It was first presented in the MODFLOW context in MODFLOW-NWT (Niswonger and others, 2011) for regular grids and then in MODFLOW-USG (Panday and others, 2013) for unstructured grids. The Newton-Raphson formulation has many advantages for water-table problems and will often converge when the standard formulation fails to converge due to wetting and drying difficulties.

The Newton-Raphson method can be applied to a system of nonlinear equations like those resulting from discretization of the unconfined groundwater flow equation. Like the standard formulation, the NewtonRaphson formulation also uses iteration, involving repeated solution of a linearized system of equations, to handle nonlinear problems. For a system of equations, the following matrix equation expresses the NewtonRaphson method

$$
\mathbf{J}^{k-1} \Delta \mathbf{h}^{k}=-\mathbf{r}^{k-1}
$$

where $\mathbf{J}$ is the Jacobian matrix calculated using heads from the previous iteration, $k-1, \Delta \mathbf{h}^{k}$ is the head upgrade vector that is equal to head difference between iteration $k$ and $k-1$, and $\mathbf{r}$ is the residual vector calculated using the CVFD equation and the heads from iteration $k-1$. The solution of equation 2-25 requires finding a new head upgrade vector, which when multiplied by the Jacobian (calculated from a previous iteration) will offset residuals calculated for the previous iteration.

As shown by Niswonger and others (2011) and Panday and others (2013), equation 2-25 can be rearranged in terms of $\mathbf{h}^{k}$ to give

$$
\mathbf{J}^{k-1} \mathbf{h}^{k}=-\mathbf{r}^{k-1}+\mathbf{J}^{k-1} \mathbf{h}^{k-1} .
$$

Equation 2-26 provides an expression for the Newton-Raphson linearized groundwater flow equation in terms of head, instead of the head upgrade. By writing the equation in this manner, it is easier to write the program so that the groundwater flow equation can be solved using either the standard formulation or the NewtonRaphson formulation without a need to have significantly different implementations. Equation 2-26 has a similar form as equation 2-24 in that the Jacobian matrix on the left-hand side and the terms on the right-hand side are known, and that head is the dependent variable for which a solution is sought. 
Application of equation 2-26 requires an expression for the residual of cell $n$. The residual for cell $n$ is calculated as the sum of inflows and outflows across each face $\left(\sum_{m \in \eta_{n}} Q_{n, m}\right)$, the inflows and outflows from sources and sinks $\left(Q_{n, s}\right)$, and changes in storage $\left(Q_{S T O}\right)$ :

$$
r_{n}=\sum_{m \in \eta_{n}} Q_{n, m}+Q_{n, s}-Q_{S T O}
$$

The Jacobian matrix is a sparse matrix that contains the partial derivative of the residual $r_{n}$ with respect to head. The diagonal of the Jacobian matrix contains terms that represent the change in the residual for cell $n$ with the change in the head for cell $n$,

$$
J_{n, n}=\frac{\partial r_{n}}{\partial h_{n}}=\sum_{m \in \eta_{n}} \frac{\partial Q_{n, m}}{\partial h_{n}}+\frac{\partial Q_{n, s}}{\partial h_{n}}-\frac{\partial Q_{S T O}}{\partial h_{n}} .
$$

Off-diagonal positions in the Jacobian matrix contains the change in the residual for cell $n$ with respect to the change in the head at connected cells, expressed as,

$$
J_{n, m}=\frac{\partial r_{n}}{\partial h_{m}}=\frac{\partial Q_{n, m}}{\partial h_{m}} .
$$

Application of equation 2-26 to equations 2-27 to 2-29 yield the following Newton-Raphson expansion of the CVFD equation for cell $n$,

$$
\begin{array}{r}
\left(\sum_{m \in \eta_{n}} \frac{\partial Q_{n, m}}{\partial h_{n}}+\frac{\partial Q_{n, s}}{\partial h_{n}}-\frac{\partial Q_{S T O}}{\partial h_{n}}\right) h_{n}^{k}+\sum_{m \in \eta_{n}} \frac{\partial Q_{n, m}}{\partial h_{m}} h_{m}^{k}=-\left(\sum_{m \in \eta_{n}} Q_{n, m}+Q_{n, s}-Q_{S T O}\right)+ \\
\left(\sum_{m \in \eta_{n}} \frac{\partial Q_{n, m}}{\partial h_{n}}+\frac{\partial Q_{n, s}}{\partial h_{n}}-\frac{\partial Q_{S T O}}{\partial h_{n}}\right) h_{n}^{k-1}+\sum_{m \in \eta_{n}} \frac{\partial Q_{n, m}}{\partial h_{m}} h_{m}^{k-1} .
\end{array}
$$

Equation 2-30 is written so that the terms are in direct correspondence to the terms in equation 2-26. The terms on the left side of equation 2-30 represent $\mathbf{J}^{k-1} \mathbf{h}^{k}$; the first term on the right side of equation 2-30 represents the negative residual, $-\mathbf{r}^{k-1}$, which is simply the sum of the inflows and outflows calculated using the heads from the previous iteration; and, the last two terms on the right side of equation 2-30 represent $\mathbf{J}^{k-1} \mathbf{h}^{k-1}$.

The terms in equation 2-30 are assembled into the matrix equations using a package approach. The form of equation 2-30 is designed to show how a package adds its individual terms to the matrix equations. As an example, the $Q_{S T O}$ term is shown in three places in equation 2-30-once in the coefficient for $h_{n}^{k}$ and twice on the right side.

\section{No-Flow and Constant-Head Cells}

In practice, formulating an equation of the form of equation 2-20 for every cell in a model grid is generally unnecessary because the status of certain cells is specified in advance to simulate the boundary conditions of the problem. In MODFLOW 6, cells used to simulate boundary conditions are grouped into two 


\section{2-16 Documentation for the MODFLOW 6 Groundwater Flow Model}

categories- "constant-head" cells and "no-flow" cells. Constant-head cells are those for which the head is specified for each time, and the head value does not change as a result of solving the flow equations. No-flow cells are those for which no flow into or out of the cell is permitted. As described in the next chapter, no-flow cells are handled differently in MODFLOW 6 from those in previous MODFLOW versions. The remaining cells of the grid, termed "variable-head" cells in this report, are characterized by heads that are unspecified and free to vary during the simulation. An equation of the form of equation 2-20 must be formulated for each constant-head and variable-head cell in the grid, and the resulting system of equations must be solved simultaneously for each time step in the simulation.

Constant-head and no-flow cells are used in MODFLOW 6 to represent conditions along various hydrologic boundaries inside the grid. For example, figure 2-9 shows the map of an aquifer boundary superimposed onto a grid of cells generated for the model. The aquifer is of irregular shape, whereas the model grid is rectangular in outline. In areas where the aquifer is coincident with the outside edge of the grid, no special designation is needed to indicate no flow because MODFLOW 6 does not compute intercell flow through the outside edges of the grid, including the top and bottom. Within the grid, no-flow cells have been used to delete the part of the grid beyond the aquifer boundary. The figure also shows constant-head cells along one section of the boundary; these may be used, for example, where the aquifer is in direct contact with major surface-water features. Other boundary conditions, such as areas of constant inflow or areas where inflow varies with head, can be simulated through the use of external source terms (chapters 6 and 7) or through a combination of noflow cells and external source terms. If flow occurs to or from the outside of the grid where the aquifer extends to the edge of the grid, then the flow can be simulated by using external source terms in the cells at the edges of the grid.

\section{Solution Procedure}

The solution procedure is controlled by the MODFLOW 6 framework. This section summarizes transient and steady-state GWF Model simulations and the role of iteration in obtaining solution results.

\section{Transient Simulations}

The objective of transient simulation is generally to predict head distributions at successive times, given the initial head distribution, the boundary conditions, the hydraulic parameters, and the external stresses. The initial-head distribution provides a value of head at each point in the grid; that is, the initial head provides the values of head at the beginning of the first of the discrete time steps into which the time axis is divided in the finite-difference process. The first step in the solution process is to calculate values of head at $t_{1}$, which marks the end of the first time step. When these have been obtained, the process is repeated to obtain heads at time $t_{2}$, the end of the second time step. To do this, equations 2-21 through 2-23 are reapplied, now using the heads from $t_{1}$ as $H O L D$. Again, a system of equations is formulated, where the unknowns are now the heads at time $t_{2}$, and this set of equations is solved simultaneously to obtain the head distribution at time $t_{2}$. This process is continued for as many time steps as necessary to cover the time range of interest.

The set of finite-difference equations is reformulated at each time step; that is, at each time step there is a new system of simultaneous equations to be solved. The heads at the end of the time step make up the unknowns for which this system must be solved; the heads at the beginning of the step are among the known terms in the equations. The solution process is repeated at each time step, yielding a new array of heads for the end of the step.

\section{Steady-State Simulations}

The flow equation, equation 2-20, was developed assuming transient conditions; however, the transient flow equation becomes the steady-state flow equation when the storage term is zero. The resulting equation 


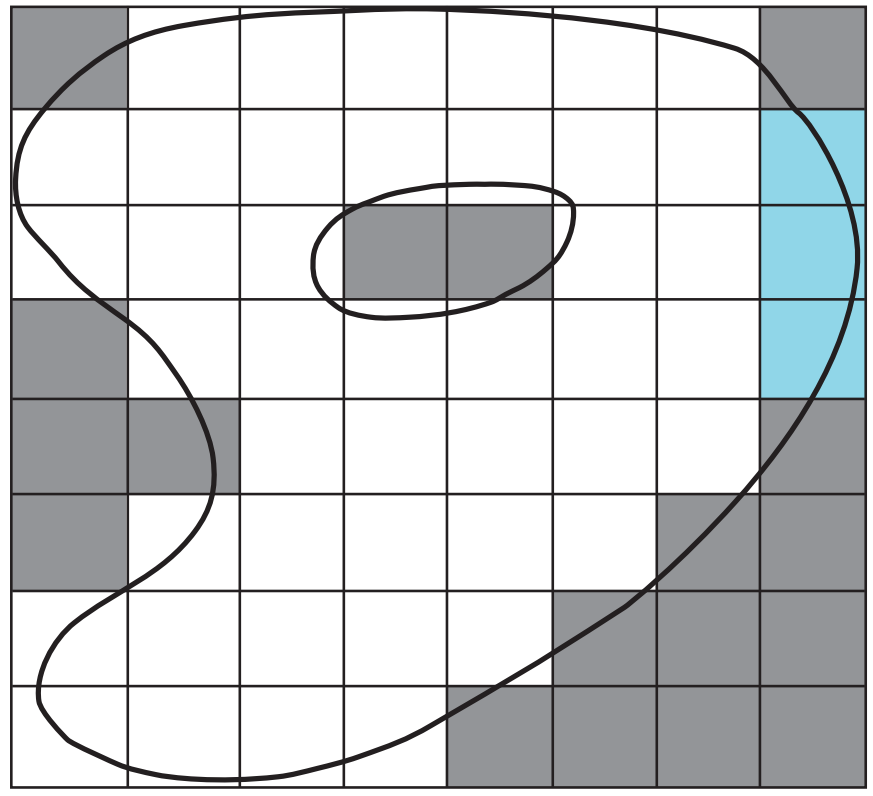

EXPLANATION

No-flow cell

Constant-head cell

Variable-head cell

Aquifer boundary

Figure 2-9. Diagram of a discretizated aquifer showing boundaries and cell designations. Figure modified from Harbaugh (2005). 


\section{2-18 Documentation for the MODFLOW 6 Groundwater Flow Model}

specifies that the sum of all inflows (where outflow is a negative inflow) from adjacent cells and external stresses must be zero for each cell in the model. A steady-state problem requires a single solution of simultaneous equations, rather than a solution for multiple time steps. Recall that an initial head was required in a transient simulation to calculate the time derivative for the first time step. For steady-state simulations, there is no direct requirement for initial head because the time derivative is removed from the flow equation. In practice, however, initial head is required for steady-state simulations because an iterative solution is used. As described next, iterative solution works by successively improving the estimated answer; therefore, an initial estimate is required to start the iterative process. In MODFLOW 6 and previous MODFLOW versions, the user-specified initial head is used as this initial estimate. The initial estimate should normally have no effect on the solution to the steady-state flow equation (unless cell wetting and drying occurs), but it may affect the number of iterations required to obtain an acceptable approximation of the solution.

\section{Iteration}

MODFLOW 6 utilizes iterative methods to obtain the solution to the system of CVFD equations for each time step. Iterative methods are used for both the standard formulation and Newton-Raphson formulation. With iterative methods, the calculation of head values for the end of a given time step is started by arbitrarily assigning a trial value, or estimate, for the head at each node at the end of that step. A procedure of calculation is then initiated that alters these estimated values, producing a new set of head values that are in closer agreement with the system of equations. These new, or interim, head values then take the place of the initially assumed heads, and the procedure of calculation is repeated, producing a third set of head values. This procedure is repeated successively at each stage, producing a new set of interim heads that more nearly satisfies the system of equations. Each repetition of the calculation is termed an "iteration." Ultimately, as the interim heads approach values that would exactly satisfy the set of equations, the changes produced by succeeding stages of calculation become very small. This behavior is utilized in determining when to stop iteration, as discussed in a subsequent paragraph.

During calculations for a time step, interim head distributions are generated in succession, and each distribution contains one interim head value for each active node in the grid. In figure $2-10$, these head distributions correspond to three-dimensional lattices, each identified by the symbol $h$ bearing two superscripts. In the program, head distributions are stored in one-dimensional vectors. The first superscript indicates the time for which the heads in the vector are calculated, whereas the second indicates the number, or level, of the iteration that produced the heads. Thus, $h^{t_{1}, 1}$ represents the vector of head values computed in the first iteration for time step $1 ; h^{t_{1}, 2}$ would represent the vector of head values computed in the second iteration, and so on. The head values that were initially assumed for time step 1 , to begin the process of iteration, appear in the head vector designated as $h^{t_{1}, 0}$. In the example of figure 2-10, multiple iterations are required to achieve closure for the heads at the end of the time step; thus, the head values for any iteration along the way for time $t_{1}$ is designated as $h^{t_{1}, k}$. Figure 2-10 also shows the vector of final head values for the end of the preceding time step $h^{t_{0}}$. The head values in this vector appear in the storage term of equation 2-20; that is, they are used in the term $\mathrm{HOLD}_{n}$ on the right side of equation 2-20 in the calculation of heads for time $t$. Because they represent heads for the preceding time step for which computations have already been completed, they appear as predetermined constants in the equation for time $t$; thus, they retain the same value in each iteration of the time step. Similarly, the final values of head for time $t_{1}$ are used as constants in the storage term during calculations for time $t_{2}$.

Ideally, one would like to specify that iteration stop when the calculated heads are suitably close to the exact solution. Because the actual solution is unknown, however, an indirect method of specifying when to stop iterating must be used. The method most commonly employed is to specify that the changes in computed heads occurring from one iteration level to the next must be less than a certain quantity, termed the "closure criterion" or "convergence criterion," which is specified by the user. After each iteration, absolute values of 


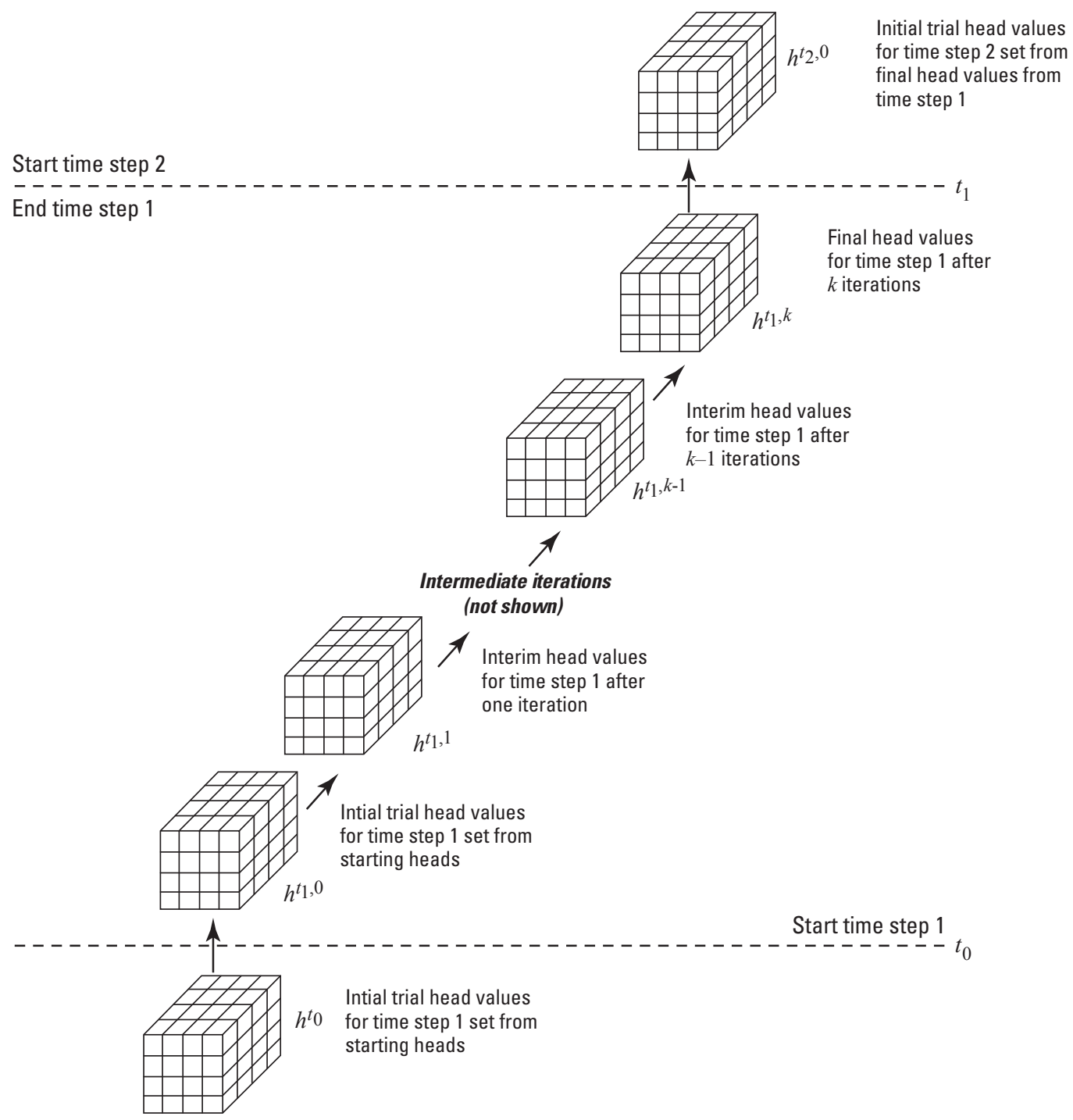

Figure 2-10. Diagram showing iterative calculation of a head distribution. Figure modified from Harbaugh (2005). 


\section{2-20 Documentation for the MODFLOW 6 Groundwater Flow Model}

change in computed head (calculated as the difference in head between the current and previous iteration) in that iteration are examined for all nodes in the grid. The largest of these absolute head-change values is compared with the closure criterion. If this largest value exceeds the closure criterion, iteration continues; if this value is less than the closure criterion, iteration is said to have "closed" or "converged," and the process is terminated for that time step. Normally, this method of determining when to stop iteration is adequate. Note that the closure criterion refers to change in computed head, and that values of head are not themselves necessarily calculated to a level of accuracy comparable to the closure criterion. As a rule of thumb, it is wise to use a value of closure criterion that is an order of magnitude smaller than the level of accuracy desired in the head results.

MODFLOW 6 also incorporates a maximum permissible number of iterations per time step. If closure is not achieved within this maximum number of iterations and the user has not set an option to continue without convergence, then the iterative process is terminated and a corresponding message is printed in the output.

The initial estimates of head used in the solution for time $t_{1}$ of figure $2-10$ could be assigned arbitrarily, or they could be chosen according to a number of different conventions. Theoretically, the iterative process would eventually converge to the same result, regardless of the choice of initial head values, although the work required would be much greater for some choices than for others. Heads computed for the end of each time step are used as the initial trial values of head for the succeeding time step. Thus, in figure $2-10$, the head vector $h^{t_{1}, k}$ contains the final estimates of head obtained after $k$ iterations for the end of time step 1 . When the calculations for step 1 are complete, these same values of head are transferred to the head vector $h^{t_{2}, 0}$ and used as the initial estimates, or trial values, for the heads of time step 2. Head values for the first time step in the simulation are assumed initially to be equal to the heads specified by the user for the beginning of the simulation.

Discussions of the mathematical basis of various iterative methods can be found in many standard references, including Saad (2003), Barrett and others (1994), Peaceman (1977), Crichlow (1977), Remson and others (1971), and Wang and Anderson (1982). The reader is advised to review one of these discussions, both to clarify general concepts and to provide an introduction to such topics as the use of matrix notation, the role of iteration parameters, and the influence of various factors on rate of convergence. In particular, such a review is recommended prior to reading about the MODFLOW 6 solution techniques in Hughes and others (2017).

An iterative procedure yields only an approximation to the solution of the system of CVFD equations for each time step; the accuracy of this approximation depends upon several factors, including the closure criterion that is employed. Even if exact solutions to the set of finite-difference equations were obtained at each step, these solutions would themselves be only an approximation to the solution of the differential equation of flow (eq. 2-2). The truncation error is defined as the discrepancy between the head given by the solution to the system of difference equations for a given node and time and the head $h(x, y, z, t)$, which would be given by the formal solution of the differential equation for the corresponding point and time. In general, truncation error tends to become greater as the grid spacing and time-step length are increased. The reader also should recognize that even if a formal solution of the differential equation could be obtained, it would be only an approximation to conditions in the field, in that hydraulic conductivity and specific storage are seldom known with accuracy, and uncertainties with regard to hydrologic boundaries are generally present.

In the remainder of the document, the superscripts denoting the time step and the iteration $(t, k)$ will generally be presented in terms of the iteration $(k)$ to simplify the symbology. The variable $t_{\text {old }}$ will always be used to represent the previous time step and values at the previous time step. Where appropriate, $H O L D_{n}$ will be used to refer to the groundwater head at the end of the previous time step (equivalent to $h^{t_{\text {old }}}$ ). 


\section{Chapter 3. Spatial Discretization}

As described in chapter 2, space is discretized by a grid of cells. The GWF Model in MODFLOW 6 provides three different packages for entering the grid information: the structured Discretization (DIS) Package, the Discretization by Vertices (DISV) Package, and the Unstructured Discretization (DISU) Package. Only one Discretization Package can be specified for a GWF Model. The DIS Package is used to represent the traditional finite-difference grid of previous MODFLOW versions in which cells are identified by their layer, row, and column position in the grid. These grids are called regular MODFLOW grids. The DISV Package is a new discretization format developed for MODFLOW 6. With the DISV Package, the user enters a list of twodimensional $x, y$ vertex pairs, and then for each two-dimensional cell, a list of vertex numbers is provided. The DISV Package may contain multiple layers, but the grid of cells is the same for each layer. The DISU Package is the most general of the three packages and requires that the user specify cell information and information about how cells are connected. The DISU Package is based on the DISU Package of MODFLOW-USG. The discretization information stored in the DIS, DISV, and DISU Packages is used during the simulation by the GWF Model to calculate the hydraulic conductance between connected cells, to calculate cell volumes for storage calculations, or to convert the recharge and evapotranspiration fluxes from dimensions of $L / T$ to $L^{3} / T$, for example. The DIS, DISV, and DISU Packages are described in this chapter.

The Discretization Packages contain information about cells such as the cell top and bottom elevations and cell dimensions or areas (in plan view). The Discretization Packages also define cell connectivity. Cell connectivity describes how model cells are connected to one another and the geometric properties of the connections. For the DIS Package, cell connectivity is implicitly described by the grid structure, cell dimensions in the row and column directions, and cell top and cell bottom elevations. For the DISV Package, cell connectivity in the horizontal direction is determined by the vertices used to define cells. Two consecutive vertices listed for a cell define an edge. If two cells share an edge, then the cells are connected. Vertical connections for the DISV Package are easily determined by the layered structure of the grid. For the DISU Package, the user must provide information about which cells are connected as well as the geometric properties of those connections.

In a GWF Model, cells are identified by their cellid, and the form of the cellid depends on the type of Discretization Package used. For the traditional finite-difference grid of the DIS Package, called regular MODFLOW grids, cells are identified by layer, row, and column. Thus, the cellid is written as (layer, row, column). When the DISV Package is used, cells are identified by the layer number and the cell number within the layer. Thus, the cellid is written as (layer, $n$ ), where $n$ is the cell number within the layer. As with the traditional finite-difference grid, it is easy to identify overlying and underlying cells with the DISV Package, as cell $(3,789)$ is overlain by cell $(2,789)$, and underlain by cell $(4,789)$, for example. When the DISU Package is used, cells are identified only by their cell number (often referred to as the node number). Thus, the cellid is written as $(n)$, where $n$ is the cell number within the grid. It is important to note that the Discretization Package type determines how cells are identified in all other packages, and that changing the Discretization Package type will require that many of the other packages be reconstructed so that cells are referenced using the appropriate cellid.

For most applications, users are encouraged to use the traditional, rectilinear finite-difference grid defined by the DIS Package. Working with layers, rows, and columns has many advantages for preprocessing and postprocessing, for identifying errors in model input, and for overall ease of use. Furthermore, the traditional finite-difference grid generally follows CVFD geometric requirements for cell connections, except in instances where horizontally adjacent cells have large vertical offsets. Therefore, accuracy issues related to the CVFD geometric requirements are less of a concern for the traditional finite-difference grid than for many other grid types. The DISV Package offers more flexibility for defining cells in the horizontal plane than the DIS Package. This flexibility can be used to create layered triangular, quadtree, and Voronoi polygon grids, for example. When designed properly, these grid types can be useful for increasing the level of resolution near hydrologic features of interest. The DISV Package is layered, which means that cells are grouped into layers. For 


\section{3-2 Documentation for the MODFLOW 6 Groundwater Flow Model}

the DISV Package, this also means that a cell can connect to only one overlying cell and one underlying cell. The layered implementation in DISV also requires that in any horizontal direction, a cell can connect to only one other cell (vertically staggered connections, where a cell connects with more than one cell in the same horizontal direction, are not allowed). The DISU Package is the most flexible of the spatial discretization packages described in this report. In some cases where a layered grid cannot be used, the DISU Package may be required. In these cases, users must be cognizant of the CVFD geometric requirements to ensure that simulation results are accurate.

\section{Regular MODFLOW Grids-Structured Discretization Package}

A regular MODFLOW grid is specified using the structured Discretization (DIS) Package. A regular MODFLOW grid is assumed to be rectangular horizontally, while the grid can be distorted vertically (fig. 31). The number of rows and columns are specified in variables $N R O W$ and $N C O L$, respectively. The horizontal size of cells is defined by variables $D E L R$ and $D E L C$. The variable $D E L R$ has one value for every column in the grid (fig. 3-1A) and is therefore of size $N C O L$. This width of a specified column is the same for all rows. Similarly, $D E L C$ has one value for every row in the grid and is therefore of size $N R O W$. The width of a row is the same for all columns.

Some users of MODFLOW have reported confusion over $D E L R$ and $D E L C$. They wrongly interpret $D E L R$ to represent distance "between" rows rather than distance "along" rows. The variable $D E L R$ is the cell size in the $x$ direction if the grid for a layer is imposed on a Cartesian coordinate system. Likewise, $D E L C$ is the cell size in the $y$ direction.

The number of layers in the grid is specified in variable $N L A Y$. Layers are numbered starting from the top layer and going down (fig. 3-1B). Cell elevations are specified in variable BOTM. Elevation of the top of layer 1 is specified in addition to the bottom elevation of every layer. The elevation information is used to calculate the thickness of all cells. The top elevation of model layers below the top layer need not be separately specified because the top elevation is the same as the bottom elevation of the layer that is directly above. Instead, all confining units must be explicitly represented using layers. Use of distorted layers, as shown in figure $3-1 B$, is not required. A flat layer is indicated by simply specifying the same value for the bottom elevation of all the cells in the layer.

Although the grid information provided by the user is in a structured format, the MODFLOW 6 program immediately converts the grid information into an unstructured format after the DIS Package is read. Internal to the MODFLOW 6 program, therefore, everything is stored in an unstructured format. Information is converted back into the structured format when output is written.

\section{Discretization by Vertices Package}

MODFLOW 6 includes a new type of discretization approach that has not been available in any other MODFLOW variants. This new approach, which is implemented in the Discretization by Vertices (DISV) Package, allows cells to be defined using two-dimensional $x, y$ pairs called vertices. In the present implementation, a two-dimensional plan-view grid is defined using vertices, and multiple layers of the twodimensional grid can be used to discretize the aquifer in the vertical direction. Cells are referred to by their two-dimensional cell number and their layer number.

Cells can be connected vertically or horizontally. In the vertical direction, a cell is connected to the cell in the layer above it (if it exists) and the cell in the layer below it (if it exists). These underlying and overlying cells are easily identified because they share the same cell number. In the horizontal direction, cells are connected if they share an edge. An edge is a line segment defined by two vertices. Cells that share an edge are automatically connected by the program. Two connected cells cannot share more than one edge. 


\section{A. Plan view}
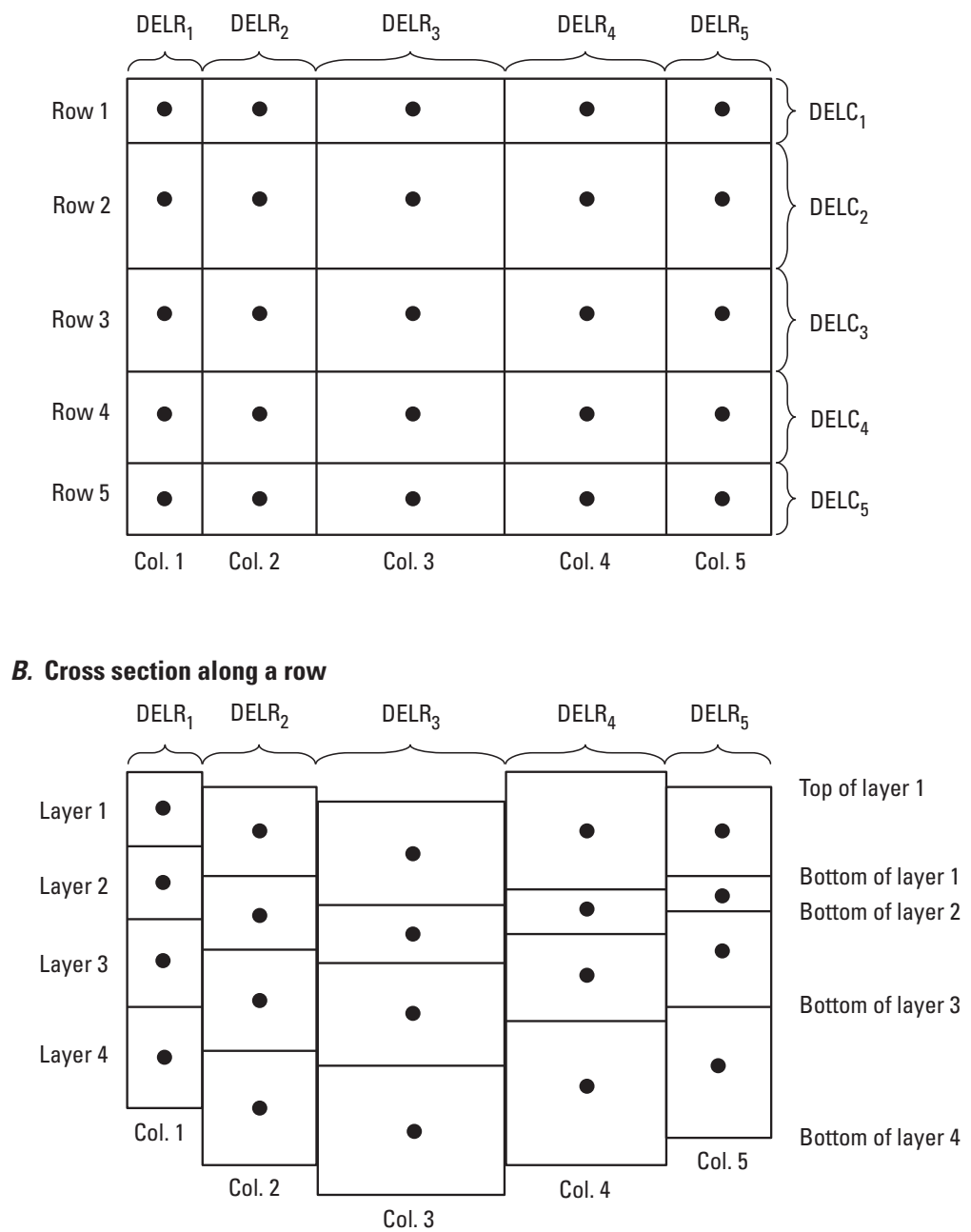

Figure 3-1. Diagram showing a regular MODFLOW grid. $A$, plan view, and $B$, cross-section view. Figure modified from Harbaugh (2005). 


\section{3-4 Documentation for the MODFLOW 6 Groundwater Flow Model}

\section{A. Quad-based grid}

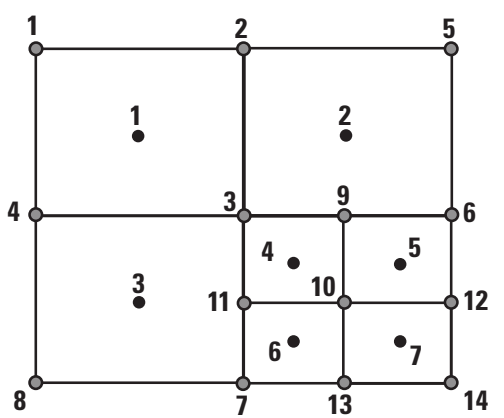

Cell NVERT Vertices

$\begin{array}{ll}4 & {\left[\begin{array}{llll}1 & 2 & 3 & 4\end{array}\right]} \\ 5 & {\left[\begin{array}{lllll}2 & 5 & 6 & 9 & 3\end{array}\right]} \\ 5 & {\left[\begin{array}{lllll}4 & 3 & 11 & 7 & 8\end{array}\right]} \\ 4 & {\left[\begin{array}{lllll}3 & 9 & 10 & 11\end{array}\right]} \\ 4 & {\left[\begin{array}{lllll}9 & 6 & 12 & 10\end{array}\right]} \\ 4 & {\left[\begin{array}{lllll}11 & 10 & 13 & 7\end{array}\right]} \\ 4 & {\left[\begin{array}{lllll}10 & 12 & 14 & 13\end{array}\right]}\end{array}$

B. Triangular grid

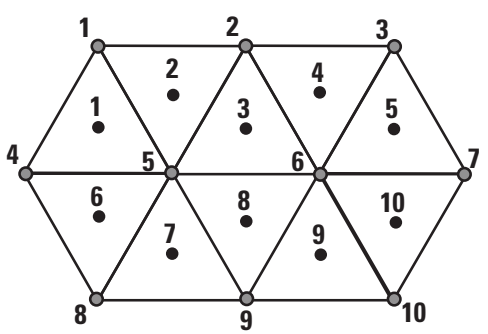

Cell NVERT Vertices

\begin{tabular}{|c|c|c|}
\hline 1 & 3 & {$\left[\begin{array}{lll}1 & 5 & 4\end{array}\right]$} \\
\hline 2 & 3 & {$\left[\begin{array}{lll}1 & 2 & 5\end{array}\right]$} \\
\hline 3 & 3 & {$\left[\begin{array}{lll}2 & 6 & 5\end{array}\right]$} \\
\hline 4 & 3 & {$\left[\begin{array}{lll}2 & 3 & 6\end{array}\right]$} \\
\hline 5 & 3 & {$\left[\begin{array}{lll}3 & 7 & 6\end{array}\right]$} \\
\hline 6 & 3 & {$\left[\begin{array}{lll}4 & 5 & 8\end{array}\right]$} \\
\hline 7 & 3 & {$\left[\begin{array}{lll}5 & 9 & 8\end{array}\right]$} \\
\hline 8 & 3 & {$\left[\begin{array}{lll}5 & 6 & 9\end{array}\right]$} \\
\hline 9 & 3 & {$\left[\begin{array}{ll}6 & 1\end{array}\right]$} \\
\hline & 3 & \\
\hline
\end{tabular}

\section{EXPLANATION}

2 - Cell center and cell number

4 . Vertex and vertex number

Figure 3-2. Diagram showing the vertices and cells defined using the Discretization by Vertices Package.

Simple examples for two types of grids are shown in figure 3-2. Figure 3-2A shows the cell and vertex numbering scheme for a quad-based grid. In this case, there are seven two-dimensional cells, which are defined by 14 vertices. The table beneath the grid shows the cell number, the number of vertices for the cell ( NVERT), and a list of the vertices. As an example, cell 1 is defined by 4 vertices: 1, 2, 3, and 4 . One requirement of the DISV Package is that additional vertices may be required to define a cell, as indicated by the vertices used to define cells 2 and 3. Cell 2 is defined by vertices 2, 5, 6, 9, and 3. Although vertex 9 does not provide added information for defining the shape of cell 2, it is required by the DISV Package to define edges that it shares with cells 4 and 5 so that cell connections can be determined. Another requirement of the DISV Package is that vertices must be listed in a clockwise order as viewed from above; however, it does not matter which vertex is listed first for a cell. Figure $3-2 B$ shows cell and vertex numbering for a triangular grid.

In the examples shown in figure 3-2, the cell polygons are not closed in the sense that the first vertex number for the cell is not repeated as the last vertex number. Closing of the cell polygon by repeating the first vertex as the last vertex is not required in the present implementation. Internally within the program, however, the first vertex number is added to the end of the vertex list in order to close the polygon. Thus, users have the option for whether or not to close cell polygons.

The layer concept for the DISV Package follows the same approach implemented for the DIS Package. The number of layers in the grid is specified in variable $N L A Y$. Layers are numbered starting from the top layer and going down. Cell elevations are specified in variable BOTM. Elevation of the top of layer 1 is specified in addition to the bottom elevation of every layer. 


\section{The IDOMAIN Capability}

MODFLOW 6 includes a new capability called the IDOMAIN capability for permanently excluding cells for the duration of a simulation. There is no provision for reincorporating excluded cells later in the simulation. The IDOMAIN capability is a new feature in MODFLOW 6, and was not available in previous MODFLOW versions. This capability is available only for DIS and DISV grids; this option is not needed for a grid specified using the DISU Package, as a preprocessor could simply remove an excluded cell prior to writing the input files. The capability is activated by specifying the optional IDOMAIN array in the input file for the DIS or DISV Packages. The size of the IDOMAIN array is equal to the number of cells in the model grid. If the $I D O M A I N$ array is not provided, then all cells are included in the simulation.

The IDOMAIN array contains a code for each cell. The integer codes for IDOMAIN have the following meanings:

- IDOMAIN $>0$ - The cell is included within the simulation. Heads and flows are calculated according to the CVFD equation.

- IDOMAIN $=0$ - The cell is permanently excluded from the simulation. This type of cell behaves like a "no-flow" cell in previous MODFLOW versions, with the exception that an excluded cell can never rewet.

- IDOMAIN $<0$-The cell is excluded from the simulation and is marked as an excluded "vertical passthrough cell." A cell overlying an excluded vertical pass-through cell is connected to the cell underlying the excluded vertical pass-through cell. If there are multiple cells marked as excluded pass-through cells in a vertical stack of cells, then the first overlying cell with an IDOMAIN value greater than zero is connected to the first underlying cell with an IDOMAIN value greater than zero.

Hydraulic conductivity, storage properties, and other information provided for every cell in the grid is still read for excluded cells in the input files. For head output, a special code is written for excluded cells. Internal and external flows for excluded cells are not calculated, and so no flow information is written for excluded cells, even for vertical pass-through cells.

Use of the IDOMAIN capability to permanently exclude cells from a simulation results in an internal renumbering of cells within the MODFLOW 6 program. An internal renumbering approach is also used by MODFLOW-NWT to eliminate no-flow cells. Within the program, the renumbering results in a reduced node number for each cell. The reduced numbering scheme is not important for users of MODFLOW 6, because all input and output is written in terms of the user numbering; however, the reduced numbering scheme will likely be important for developers or others working with the source code.

Distribution of IDOMAIN code entries for an example cross-section model is shown in figure 3-3.

\section{Unstructured Discretization Package}

For a generalized unstructured grid, the user must provide cell information as well as information on cell connectivity. Information in the DISU Package is divided into three groups: grid dimensions (number of cells and number of connections), cell information, and connection information. For an unstructured grid, grid dimensions are defined by the number of cells, NODES, and the number of connections, NJA. NJA is calculated as the number of unique connections plus NODES. The connection between cell $n$ with cell $m$ is considered different (and unique) from the connection between cell $m$ and cell $n$. These dimensions define the size of the arrays read from the DISU Package for cell information and for cell connections.

Every cell in the model grid is assigned a number. This number is referred to as the cell number or the node number. For grids with cells that overlie one another, the overlying cell must always have a lower number than the underlying cell. This information is used in some cases to determine whether a connected cell is above or below. 


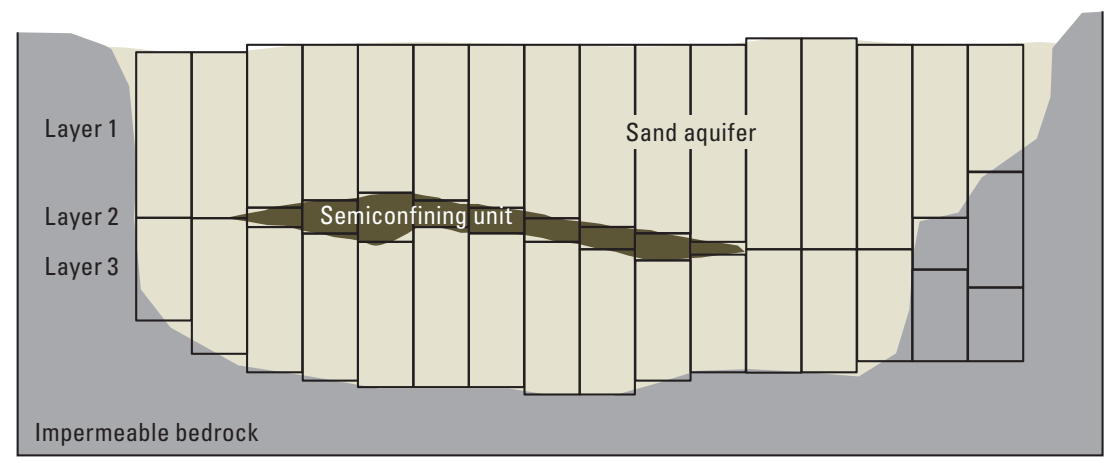

\begin{tabular}{|c|c|c|c|c|c|c|c|c|c|c|c|c|c|c|c|c|} 
Layer 1 & 1 & 1 & 1 & 1 & 1 & 1 & 1 & 1 & 1 & 1 & 1 & 1 & 1 & 1 & 1 & 1 \\
\hline Layer 2 & -1 & -1 & 1 & 1 & 1 & 1 & 1 & 1 & 1 & 1 & 1 & -1 & -1 & -1 & 0 & 0 \\
\cline { 2 - 14 } \\
\cline { 2 - 13 } 3 & 1 & 1 & 1 & 1 & 1 & 1 & 1 & 1 & 1 & 1 & 1 & 1 & 1 & 1 & 0 & 0 \\
\hline
\end{tabular}

$$
\begin{array}{cl} 
& \text { EXPLANATION } \\
& \text { IDOMAIN codes } \\
>0 & \text { Included cell } \\
=0 & \text { Excluded cell } \\
<0 & \text { Excluded pass-through cell }
\end{array}
$$

Figure 3-3. Diagram showing IDOMAIN variable in cross section for a single row or column. The sand aquifer overlies impermeable bedrock and contains a discontinuous semiconfining unit. IDOMAIN codes of -1 are assigned to the second layer in areas where the semiconfining unit is absent. The -1 IDOMAIN code indicates that the cell will be an excluded passthrough cell, in which case a vertical connection will be made between the overlying and underlying cells.

Cells defined with the DISU Package (and the DIS and DISV Packages) are required to be prismatic with horizontal tops and bottoms and vertical faces. The DISU Package requires cell top and cell bottom elevations and surface areas for every cell in the grid. This information is stored in the program as three one-dimensional arrays called TOP, BOT, and $A R E A$, respectively. Each of these arrays is of size NODES.

Cell connection information is provided in the DISU Package in the form of five or six one-dimensional arrays (table 3-1). These arrays are patterned after the arrays that are read by the DISU Package of MODFLOW-USG. Arrays that do not share an identical description with their MODFLOW-USG counterpart have been renamed to avoid confusion.

The first two arrays in table 3-1 describe cell connectivity. The $I A C$ array, which is of size NODES, contains the number of connections (plus one) for every cell in the grid. The $I A C$ array is read by the program, but it is immediately converted by the program into an index array; this index array is stored in the program as $I A$, which is of size $N O D E S$ plus 1 . In the example shown in figure 3-4, cell $n$ is connected to eight other cells, and so the value for $I A C(n)$ is 9 , which is equal to the eight connected cells plus one for cell $n$ itself. The $J A$ array contains, for each cell in the grid, a list of a list of cell numbers for which there is a connection. The $J A$ array is of size $N J A$. For example, the $J A$ entries for cell $n$ in figure 3-4 are as follows: $n, m_{1}, m$, $m_{2}, m_{3}, m_{4}, m_{5}, m_{6}$, and $m_{7}$. Note that the list for cell $n$ must contain $n$ as the first value in the list; this is consistent with MODFLOW-USG and allows the $J A$ storage scheme to work for the storage scheme required by the linear solver. The remaining cell numbers following cell $n$ must be sorted in ascending order. In this report, the set of cells connected to cell $n$ is denoted by $\eta_{n}$. Some equations use this notation to sum over all of the $m$ cells connected to cell $n$. This is written in mathematical notation as $\sum_{m \in \eta_{n}}$. Panday and others (2013) provide a thorough description of the $I A$ and $J A$ arrays, and how they are used to store cell connectivity and the underlying structure of the coefficient matrix.

The conductance values calculated by the GWF Model depend on whether the connection is horizontal or vertical. The orientation of each connection is listed in the $I H C$ array. The $I H C$ array entered by the user has 
Table 3-1. Arrays that define cell connection properties for the unstructured Discretization Package (DISU).

\begin{tabular}{|c|c|c|}
\hline Variable & Size & Description \\
\hline$I A C$ & NODES & For each cell $n$, the number of cell connections plus one. \\
\hline$J A$ & $N J A$ & For each cell $n$ a list of connected $m$ cells. \\
\hline$I H C$ & NJA & $\begin{array}{l}\text { Horizontal connection code for each } n-m \text { connection: } \\
0 \text { indicates a vertical connection, } \\
1 \text { indicates a horizontal connection, and } \\
2 \text { indicates a vertically staggered horizontal connection. }\end{array}$ \\
\hline CL12 & NJA & $\begin{array}{l}\text { For each } n-m \text { connection, the distance from the center of the cell } n \\
\text { to the face that it shares with cell } m \text {. }\end{array}$ \\
\hline$H W V A$ & NJA & $\begin{array}{l}\text { Width perpendicular to flow for a horizontal connection, or } \\
\text { the face area for a vertical connection. }\end{array}$ \\
\hline$A N G L D E G X$ & NJA & $\begin{array}{l}\text { Optional array that contains the angle (in degrees) between the user-defined } \mathrm{x} \text {-axis } \\
\text { and the outward normal to the face for the connection. }\end{array}$ \\
\hline
\end{tabular}

A. Plan view

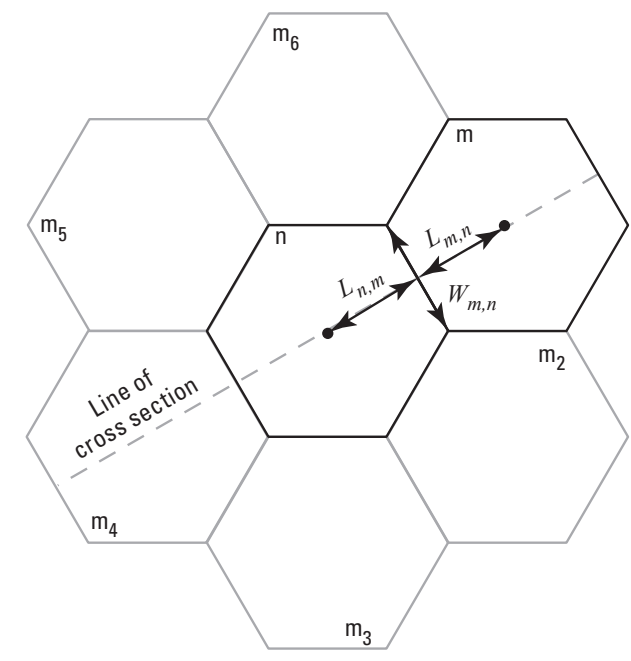

B. Cross section

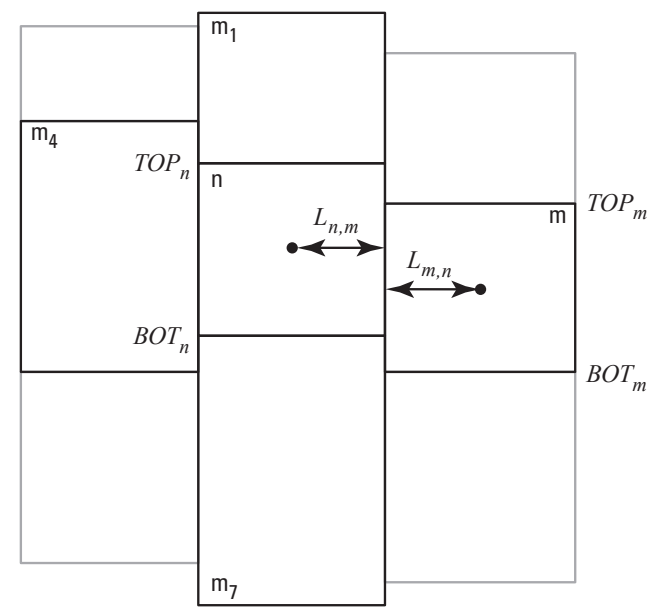

Figure 3-4. Diagram of a control-volume finite-difference grid showing connected cells and geometric lengths used in the calculation of conductance. $A$, plan view, and $B$, cross-section view. 


\section{3-8 Documentation for the MODFLOW 6 Groundwater Flow Model}

$N J A$ values within it; however, these values are symmetric in that the $I H C$ value for the $n-m$ connection is the same as the $I H C$ value for the $m-n$ connection. To conserve memory, MODFLOW 6 stores only the "upper diagonal" part of this array, meaning that $I H C$ values are only stored for an $n-m$ connection if $n$ is less than $m$. An $I H C$ value of 0 means that the connection is vertical. If the value for $n$ is less than the value for $m$, then cell $n$ overlies cell $m$. Alternatively, if $m$ is less than $n$, then cell $m$ overlies cell $n$. An $I H C$ value greater than 0 indicates that the connection is horizontal. There are two types of horizontal connections, which are denoted by values of 1 and 2 in the $I H C$ array. These values determine how the flow area is calculated for two horizontally connected cells.

An $I H C$ value of 1 assigned to the connection between two cells indicates that cells are horizontally connected using the traditional MODFLOW approach. With this approach, the full thickness of both cells is used to calculate hydraulic connection properties. For example, figure 3-5 shows a simple example of a deformed grid to illustrate the concept. In this figure, cell 8, for example, shares its right face with the left face of cells 3 , 6, and 9. An $I H C$ value of 1 indicates that the full thickness of cell 8 is used with the full thickness of cell 9 to calculate the hydraulic connection properties. Thus, when an $I H C$ value of 1 is assigned for the connection between cells 8 and 9, the user should not make connections between cells 8 and 3 and cells 8 and 6 (within the $J A$ array) even though their cell faces intersect. This traditional approach is commonly used in MODFLOW applications to represent gradually dipping and continuous layers. Steeply dipping layers generally should not be represented in this way, as vertical cell offsets result in violations of the CVFD connection requirements. Anderson and others (2015) recommend that this approach be limited to slopes less than 10 degrees.

The concept of a vertically staggered grid was introduced with MODFLOW-USG (Panday and others, 2013). With a vertically staggered horizontal connection (an $I H C$ value of 2), the top and bottom elevations for the connected cells are used to calculate the thickness of the hydraulic connection. In this case, the user should make connections (in the $J A$ array) between cells that share any part of a face. Returning to figure $3-$ 5 , the user may wish to explicitly connect cell 8 to cells 3,6 , and 9 . If this were the case, the user would add these connections to the $J A$ array and mark them as vertically staggered horizontal connections. The thickness of these connections would then be calculated according to the overlap thickness based on the top and bottom elevations. Vertically staggered horizontal connections are required to represent locally refined grids where the child model has an increased number of layers, for example. Chapter 4 describes the calculation of hydraulic connection properties for these horizontal and vertically staggered horizontal connection alternatives.

The CL12 array contains the distance between the center of a cell and the face that it shares with a neighbor. For example, in figure 3-4, values stored in $C L 12$ correspond to $L_{n, m}$ and $L_{m, n}$. The $C L 12$ is not symmetric in that $L_{n, m}$ may not be equal to $L_{m, n}$. The order of the entries in the $C L 12$ array correspond directly with the order of the connections listed in the $J A$ array.

The $H W V A$ array contains the flow width if the connection is horizontal and the flow area if the connection is vertical. Like the $I H C$ array, the $H W V A$ array is also symmetric and so only the "upper diagonal" part of the array is stored during the simulation. For a vertical connection, the user should calculate the intersection area of cells $n$ and $m$ and assign this intersection area to $H W V A$. For a horizontal connection $(I H C>0)$, $H W V A$ represents the horizontal width available for flow $\Delta w_{n, m}$ between cells $n$ and $m$. For a vertical connection $(I H C=0), H W V A$ represents the area available for flow, $A_{n, m}$, between cells $n$ and $m$. $H W V A$ replaces the FAHL array of the MODFLOW-USG discretization package. The FAHL array in MODFLOWUSG is consistent in that it contains a flow area for both horizontal and vertical connections; however, use of $F A H L$ requires that it be regenerated whenever cell top and cell bottom elevations change. For this reason, $H W V A$ contains flow area for vertical connections and flow widths for horizontal connections. By doing so, the $H W V A$ does not need to be regenerated when cell top and bottom elevations are changed by the user.

The $A N G L D E G X$ array is the final array listed in table 3-1. This is an optional array; it is only required in some cases, such as when horizontal anisotropy is specified. The $A N G L D E G X$ array contains the angle between the horizontal x-axis and an outward normal on the cell face (fig. 3-6). Values for $A N G L D E G X$ are 
Chapter 3. Spatial Discretization 3-9

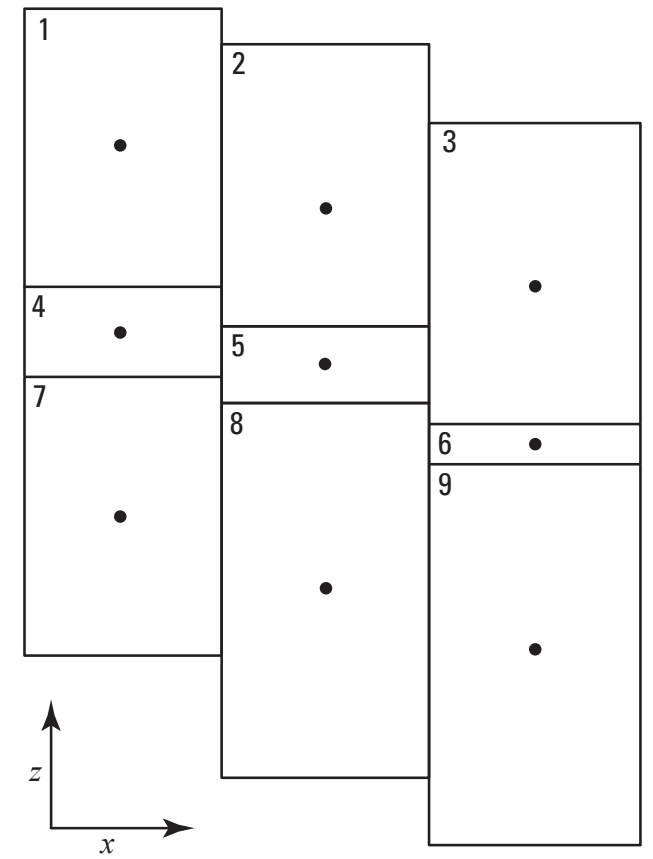

Figure 3-5. Diagram of cross section through a model grid used to demonstrate the concept of a horizontal connection and a vertically staggered horizontal connection. For a traditional MODFLOW representation in which a cell connects to only one other cell in a horizontal direction, the horizontal connection should be marked with an $I H C$ value of 1 (for example, the intent is that cell 8 is connected on its right face only to cell 9). For a vertically staggered connection in which a cell may connect to more than one cell in a horizontal direction, the horizontal connection is vertically staggered and should be marked with an $I H C$ value of 2 (for example, the intent is that cell 8 is connected on its right face to cells 3,6 , and 9). Vertically staggered horizontal connections can be specified for the unstructured Discretization (DISU) Package, but not for the structured Discretization (DIS) and Discretization by Vertices (DISV) Packages. 


\section{3-10 Documentation for the MODFLOW 6 Groundwater Flow Model}

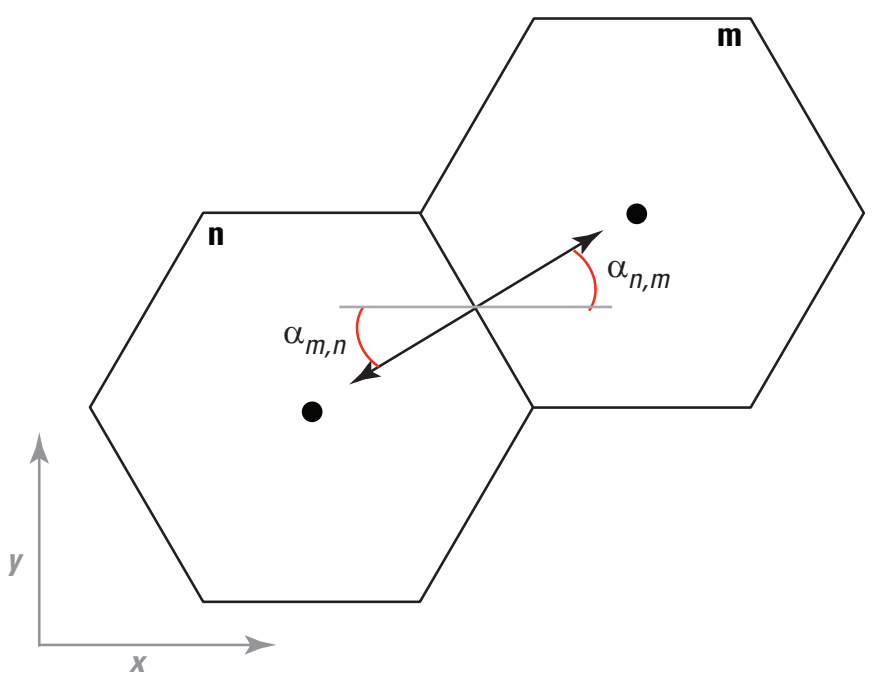

Figure 3-6. Diagram showing the angle between the $\mathrm{x}$-axis and an outward normal on the cell face. Values for $\alpha$ are provided by the user in the unstructured Discretization (DISU) Package.

provided for every connection; however, this array is also symmetric in that $\alpha_{n, m}=\alpha_{m, n}$, and so only the upper triangular part of the matrix is stored during the simulation.

\section{Model Grid and Aquifer Boundaries}

The size of the model grid is specified by the user in terms of the number of rows $(N R O W)$, number of columns $(N C O L)$, and number of layers $(N L A Y)$ for a regular grid, in terms of number of cells per layer $(N C P L)$, and number of layers $(N L A Y)$ for a discretization by vertices grid, or in terms of the number of nodes (NODES) for a DISU grid. These terms define a grid of cells in the form of a rectangular box (DIS Package) or an unstructured grid of cells in the form of a polygon (DISV and DISU Packages). In formulating the CVFD equations, cell-to-cell conductance terms are omitted for the exterior of cells on the outer surface of this grid. Thus, considering flow along a row in a regular grid, a cell-to-cell conductance term is developed for the interval between column 1 and column 2, but not for the interval to the opposite side of column 1; similarly, a conductance term is developed for the interval between the second to last and last column, but not for the interval beyond the last column. Similar conventions are established in the other two directions for a regular grid, so that, in effect, the grid is bounded externally by planes across which no cell-to-cell flow occurs. If these boundaries of the model grid, which are actually embedded in the program, coincide with impermeable boundaries in the aquifer, they can be relied upon to simulate the no-flow condition along those aquifer boundaries without further specification by the user. In general, however, the aquifer boundaries will be irregular in form, or will not be of a simple impermeable character. In these cases, the aquifer boundary must be simulated by excluding cells using the IDOMAIN capability, designing the grid to correspond to the aquifer boundary. Assignment of constant-head cells, use of external stress terms, or a combination of these may also be required to represent the aquifer boundary. While no cell-to-cell conductance terms are formulated for the interval above the uppermost layer of the model grid, flow into this layer from above is commonly represented in the model through external stress terms-for example, terms representing recharge or stream seepage. Likewise, no conductance terms are formulated for the interval below the model grid, and so there is no flow across the bottom of the model grid unless constant heads or another external stress is applied.

Where the limits of an aquifer do not coincide with the external edge of a model grid, cells may be excluded to designate parts of the grid that fall outside the aquifer boundaries; this is discussed through an example in chapter 2. As noted in the same example, constant-head cells may be used to represent such features as surface-water bodies of constant level that are in full contact with the aquifer. Boundaries that are 
characterized by a constant rate of flow into or out of the aquifer may be simulated using a no-flow boundary in conjunction with the Well Package, by assigning appropriate withdrawal or recharge rates to nodes just inside the boundary. Boundaries characterized by inflow that varies in proportion to head can be simulated using the General-Head Boundary Package or the River Package, where these again are applied to nodes just interior to a no-flow boundary. Use of the River Package would involve specifying artificial riverbed conductance and river stage (head) values at each cell along the boundary, where these values are deliberately chosen in such a way as to duplicate the required head-flow relations.

\section{Considerations for Grid Design}

The model grid may be visualized in terms of a three-dimensional assemblage of cells, each cell containing a point at a node at which head is to be calculated. The size and resolution of the model grid is specified by the user. There are many factors to consider when designing a model grid, such as the appropriate level of resolution, internal sources and sinks, heterogeneity, the presence of faults, conduits, and barriers, and the purpose of the model, for example. The design of the model grid in relation to these factors can substantially affect the accuracy of the simulation results. For example, regional groundwater models with large cells cannot be expected to produce accurate results at the local scale. An exhaustive discussion of these factors is beyond the scope of this report; however, readers can find guidance on these issues by consulting a groundwater modeling textbook, such as the one by Anderson and others (2015).

There are other accuracy issues with the grid design that users must consider. As described by Panday and others (2013) and reiterated in this report, users should be aware of the CVFD connection requirements-a line drawn between cell centers should intersect the center of the shared face at a right angle. Simple grids of regular polygons, equilateral triangles, and rectangles meet the CVFD connection requirements, but nested grids and grids consisting of nonregular polygon-shaped cells generally do not. Use of finite-element and finite-volume mesh-generation software may produce grids that conform or nearly conform to the CVFD connection requirements, but users should check to ensure this is the case. Grids consisting of cells with concave shapes are also problematic; thus, grids should be comprised of cells with convex shapes (fig. 3-7). With this flexibility in gridding, it is incumbent upon the user to ensure that the grid used to discretize the domain is appropriate for the problem geometry and flow system, and that violation of the CVFD requirement does not introduce large errors in the flow solution. Use of the GNC Package may be required for some grid types; however, errors resulting from irregular grid shapes may be difficult to quantify. Furthermore, errors in the simulated results may be large, even for converged solutions with small mass-balance errors.

There are also issues to consider with vertical discretization. At one extreme, vertical discretization can be visualized simply as an extension of areal discretization — a more or less arbitrary process of dividing the flow system into segments in the vertical dimension, governed in part by the vertical resolution desired in the results. At the opposite extreme, vertical discretization can be viewed as an effort to represent individual aquifers or permeable zones by individual layers of the model. Figure 3-8A shows a typical hydrogeologic sequence discretized in figures $3-8 B$ and $3-8 C$, according to both interpretations. The first viewpoint (fig. 3$8 B$ ) leads to rigid superposition of an orthogonal three-dimensional grid on the geohydrologic system; while there may be a general correspondence between geohydrologic layers and model layers, no attempt is made to make the grid conform to stratigraphic or structural irregularities. Under the second viewpoint (fig. 3-8C), model layer thickness is considered variable, to simulate the varying thickness of geohydrologic units; this leads, in effect, to a deformed grid.

Each of these methods of viewing the vertical discretization process has advantages, and each presents difficulties. The model equations are based on the assumption that hydraulic properties are uniform within individual cells, or at least that meaningful average or integrated properties can be specified for each cell; these conditions are more likely to be met when model layers conform to geohydrologic units as in figure 3-8C. Moreover, greater accuracy can be expected if model layers correspond to intervals within which vertical head 


\section{3-12 Documentation for the MODFLOW 6 Groundwater Flow Model}

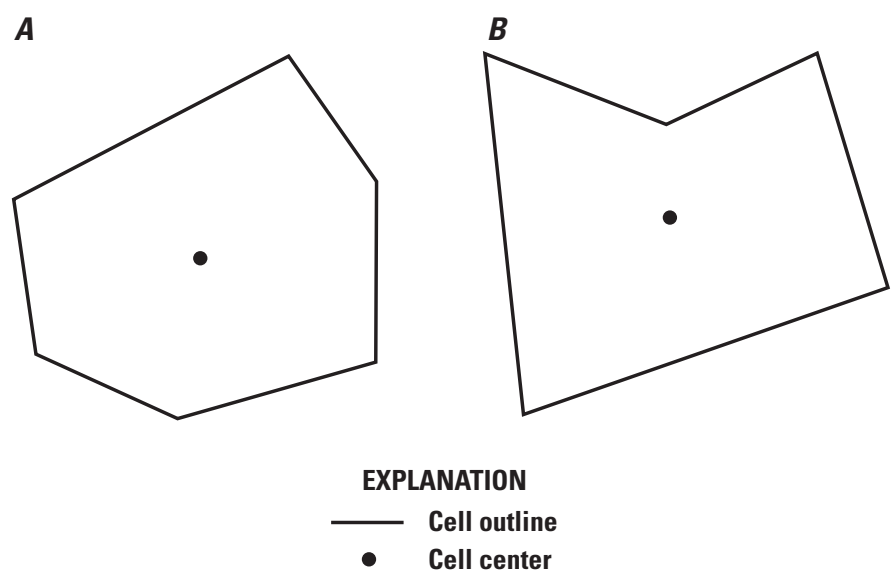

Figure 3-7. Diagram showing examples of $A$, convex, and $B$, concave polygons. A convex polygon is one in which all interior angles are less than or equal to 180 degrees. A concave polygon has at least one interior angle that is greater than 180 degrees. MODFLOW 6 cells should have a convex shape. Figure modified from Panday and others (2013).

loss is negligible, and this is also more likely under the configuration of figure $3-8 C$. On the other hand, the deformed grid of figure 3-8C fails to conform to many of the assumptions upon which the model equations are based; for example, individual cells no longer have rectangular faces, and the major axes of hydraulic conductivity may not be aligned with the model grid. Some error is always introduced by these departures from assumed conditions.

In practice, many vertical discretization schemes turn out to be a combination of the viewpoints illustrated in figures 3-8B and 3-8C. For example, even where layer boundaries conform to geohydrologic contacts, the use of more than one layer to simulate a single geohydrologic unit may be necessary simply to achieve the required model accuracy. Figure 3-9 shows a system consisting of two sand units separated by a clay unit; the units are of uniform thickness and each could be represented by a single layer without deformation of the grid. However, head at each horizontal location would be represented by a single value in each unit; if greater detail in representing the vertical head distribution in the units is required, multiple layers must be used to represent each unit. Similarly, figure 3-10 shows a sand-clay system in which pumpage from the sands is sustained partially by vertical flow of water released from storage in the clay. If the objective of analysis is to determine the pattern of storage release in the clay, several model layers would be required to represent that unit, as shown in the figure.

For many practical modeling applications, the process of discretization is complicated by aquifers and confining units that are discontinuous across an area of interest. Part of the complication stems from previous MODFLOW limitations that required each model layer to extend across the entire domain. When model layers correspond directly to aquifer units, as is the case for many regional groundwater models, it is not always clear how to assign model layer properties in areas where the hydrogeologic unit represented by the model layer is thin or does not exist. The IDOMAIN capability described in this chapter was implemented specifically to address this situation, and in some cases, may substantially improve computer run times by reducing the number of cells included in the numerical solution. Run times and solution efficiency also may improve if thin cells near the water table are removed.

Use of the DISU Package offers considerable flexibility for representing the complex structure of faults and folded hydrogeologic units. Special care must be taken when using the DISU Package to ensure that model cells do not overlap within the model domain. Because a top and bottom elevation is provided for each model cell, it is possible to have cells overlap in the vertical direction. An additional level of preprocessing may be required to ensure that this situation does not occur, or that these overlaps are minimal and do not affect the accuracy of the solution. 


\section{A. Aquifer cross section}

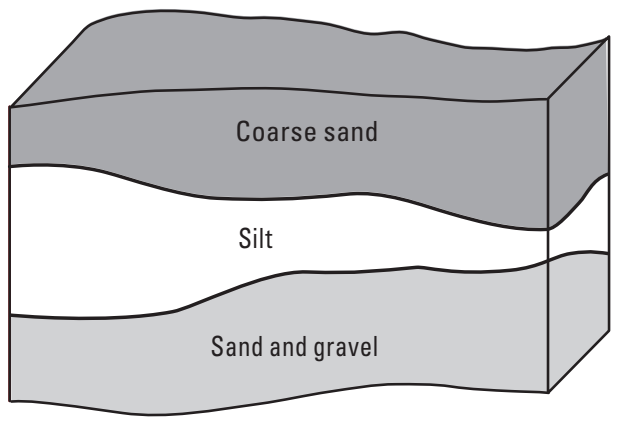

$B$. Aquifer cross section with rectilinear grid superimposed
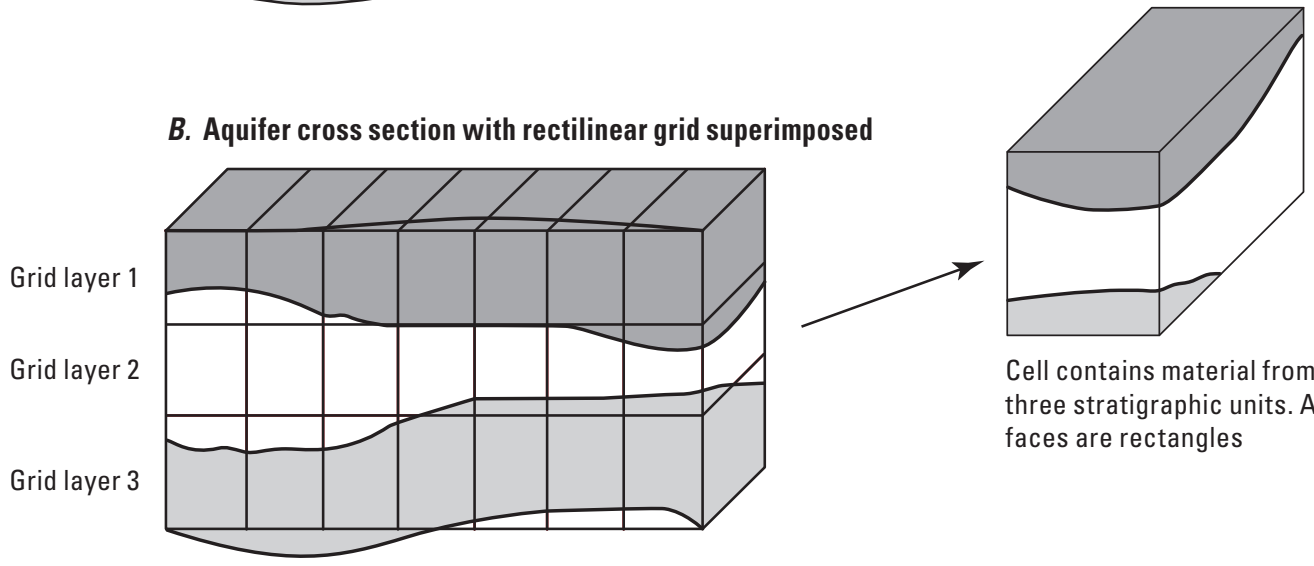

Cell contains material from three stratigraphic units. All faces are rectangles

\section{Aquifer cross section with deformed grid superimposed}

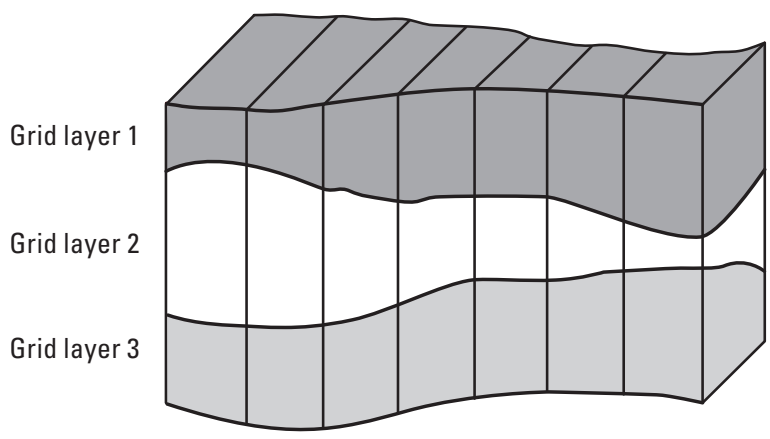

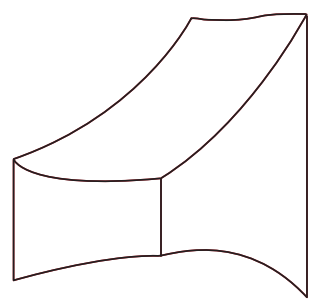

Cell contains material from only one stratigraphic unit. Faces are not rectangles

Figure 3-8. Diagram showing different schemes of vertical discretization. Figure modified from Harbaugh (2005). 


\section{3-14 Documentation for the MODFLOW 6 Groundwater Flow Model}

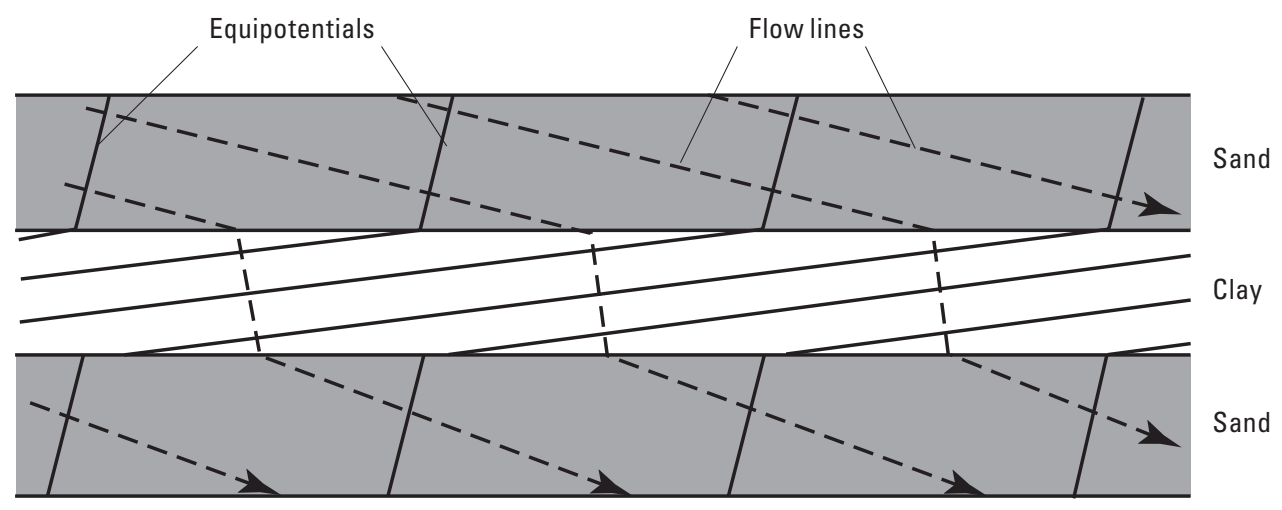

Figure 3-9. Diagram showing the possible pattern of flow in a cross section consisting of two high-conductivity units separated by a low-conductivity unit. Figure modified from Harbaugh (2005).

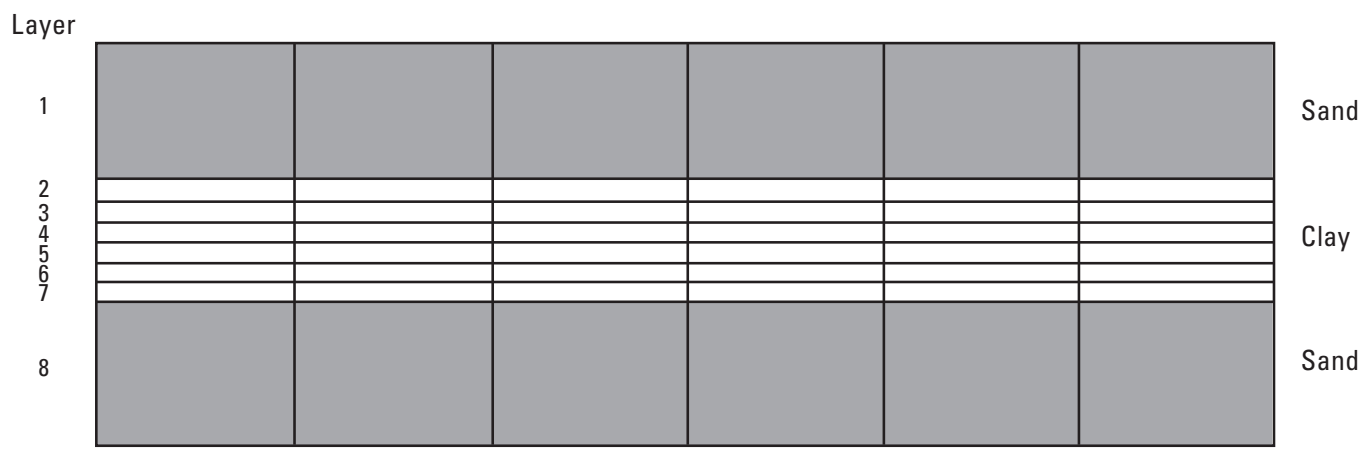

Figure 3-10. Diagram showing a cross section in which a low-conductivity unit is represented by six model layers. Figure modified from Harbaugh (2005). 
The approaches to vertical discretization described above all lead to a set of equations of the form of equation 2-21, which must be solved simultaneously at each time step. The differences among these approaches arise in the way the various conductances and storage terms are formulated and, in general, in the number of equations to be solved, the resolution of the results, and the accuracy of the results. MODFLOW 6 is capable of implementing any of these approaches to vertical discretization in that the elevations of the individual cells can vary. Chapters 4 and 5, which describe the Node Property Flow and Storage Packages, contain a discussion of the conductance and storage terms corresponding to the various ways of conceptualizing the vertical discretization. 


\section{Chapter 4. Internal Flow Packages}

This chapter documents packages that simulate internal flow in MODFLOW 6. The primary package is Node-Property Flow (NPF). There can be only one NPF Package specified for a GWF Model. This chapter also describes the Horizontal Flow Barrier (HFB) and Ghost Node Correction (GNC) Packages, which can be used to supplement NPF. Like for the NPF Package, there can be at most only one GNC Package and one HFB Package specified for a GWF model.

\section{Node Property Flow Package}

The NPF Package replaces the conductance calculation parts of the Block-Centered Flow (BCF), LayerProperty Flow (LPF), and Upstream Weighting (UPW) Packages of previous MODFLOW versions. NPF is the part of the GWF Model that calculates hydraulic conductance, both horizontal and vertical, between adjacent cells. Storage calculations, which are part of the BCF and LPF Packages in previous MODFLOW versions, have been moved into a separate Storage (STO) Package. The STO Package is independent of the NPF Package, and is, therefore, described separately in the next chapter.

The NPF Package calculates the conductance coefficients, $C_{n, m}$, in the following form of the governing CVFD equation:

$$
\sum_{m \in \eta_{n}} C_{n, m}\left(h_{m}-h_{n}\right)-P_{n} h_{n}+Q_{n}=S S_{n} A_{n} \Delta v_{n} \frac{h_{n}-H O L D_{n}}{t-t_{\text {old }}} .
$$

Equation 4-1 corresponds to equation 2-20, except that cell volume, $V_{n}$ is replaced by $A_{n} \Delta v_{n}$, where $A_{n}$ is the horizontal cell area and $\Delta v_{n}$ is the saturated thickness of cell $n$. The $h$ terms refer to the head at the end of the time step. Cell thickness, $\Delta v_{n}$, includes the subscript $n$ to indicate that cell thickness may vary from cell to cell, thus allowing for a vertically distorted grid (also called a deformed mesh) as discussed in chapters 2 and 3. For solution, equation $4-1$ is transformed into

$$
\sum_{m \in \eta_{n}} C_{n, m} h_{m}+\left(-\sum_{m \in \eta_{n}} C_{n, m}-H C O F_{n}-\frac{S S_{n} A_{n} \Delta v_{n}}{t-t_{\text {old }}}\right) h_{n}=R H S_{n}
$$

which corresponds to equation 2-21. Coefficients $P_{n}$ and $Q_{n}$ are incorporated into $H C O F_{n}$ and $R H S_{n}$, respectively, and the portion of the storage term involving $H O L D_{n}$ is incorporated into $R H S$.

\section{Confined and Convertible Cells}

The NPF Package supports two types of cells-confined and convertible. The determination for the cell type is based on the values specified for ICELLTY PE, which is provided on a cell-by-cell basis as input to the NPF Package. A value of zero indicates that the cell is confined. A nonzero value indicates that the cell is convertible. A confined cell is one in which transmissivity is constant throughout the simulation, regardless of whether or not the calculated head in the cell is above or below the cell top elevation. Transmissivity is computed from hydraulic conductivity and cell dimensions using the equations presented in this chapter. For confined cells, drying, wetting, and the vertical flow options for perched conditions are not active.

A convertible cell is one in which transmissivity varies during the simulation based on the calculated head in the cell. Transmissivity is computed during each solver iteration based on the saturated cell thickness. When the standard formulation is used, the computation of transmissivity includes the possibility for a cell convert- 


\section{4-2 Documentation for the MODFLOW 6 Groundwater Flow Model}

ing to no flow when head goes below the bottom elevation. For this case, the vertical flow correction and cell wetting capabilities can be applied if desired.

The uppermost model cells containing the water table should be marked as convertible. Also, the top elevation of these cells, which is specified in the Discretization File, should be higher than the water level will reach at any time in the simulation. This will result in a saturated thickness that is equal to the water table minus the cell bottom elevation. It is the user's responsibility in this situation to check to see that the simulated water level has not exceeded the top elevation, which would trigger the use of the top elevation instead of the water-table elevation, for calculating saturated thickness. A logical approach for specifying the top elevation for water-table cells is to set it equal to land-surface elevation. That is, in most situations the water table is not expected to exceed land-surface elevation.

\section{Saturated Cell Thickness}

Calculation of the saturated thickness is required for each cell in order to calculate hydraulic conductance between cells. The saturated thickness for a cell $\left(\Delta v_{n}\right)$ is calculated differently, depending on whether the cell is confined or convertible. For confined cells, $\Delta v_{n}$ is simply the cell thickness (vertical cell width). This can be computed internally in MODFLOW 6 using cell elevations in the discretization file:

$$
\Delta v_{n}=\left(T O P_{n}-B O T_{n}\right)
$$

For water-table conditions, $\Delta v_{n}$ depends on head. Further, a cell can convert during the simulation between a confined cell and a cell that contains the water table. The saturated thickness for water-table conditions is calculated as

$$
\Delta v_{n}= \begin{cases}\left(\operatorname{TOP}_{n}-B O T_{n}\right) & \text { if } h_{n} \geq \operatorname{TOP}_{n} \\ \left(h_{n}-B O T_{n}\right) & \text { if } T O P_{n}>h_{n}>B O T_{n} \\ 0 & \text { if } h_{n} \leq B O T_{n}\end{cases}
$$

When water-table conditions are simulated, the saturated thickness of the cell is recalculated at the start of each iteration using equation 4-4. If head drops below the aquifer bottom (eq. 4-4), the cell is considered to be fully dewatered. If the standard formulation is used, then fully dewatered cells are marked as dry and the head is set to a dry value. The model also has provision for the resaturation of fully dewatered cells. This optional provision for cell rewetting is described in a separate section in this chapter.

\section{Saturated Cell Fraction}

When the Newton-Raphson formulation is used, the abrupt transitions in equation 4-4 must be smoothed to allow calculation of partial derivatives. This is handled by using a smoothed, saturated cell fraction that is calculated using, 


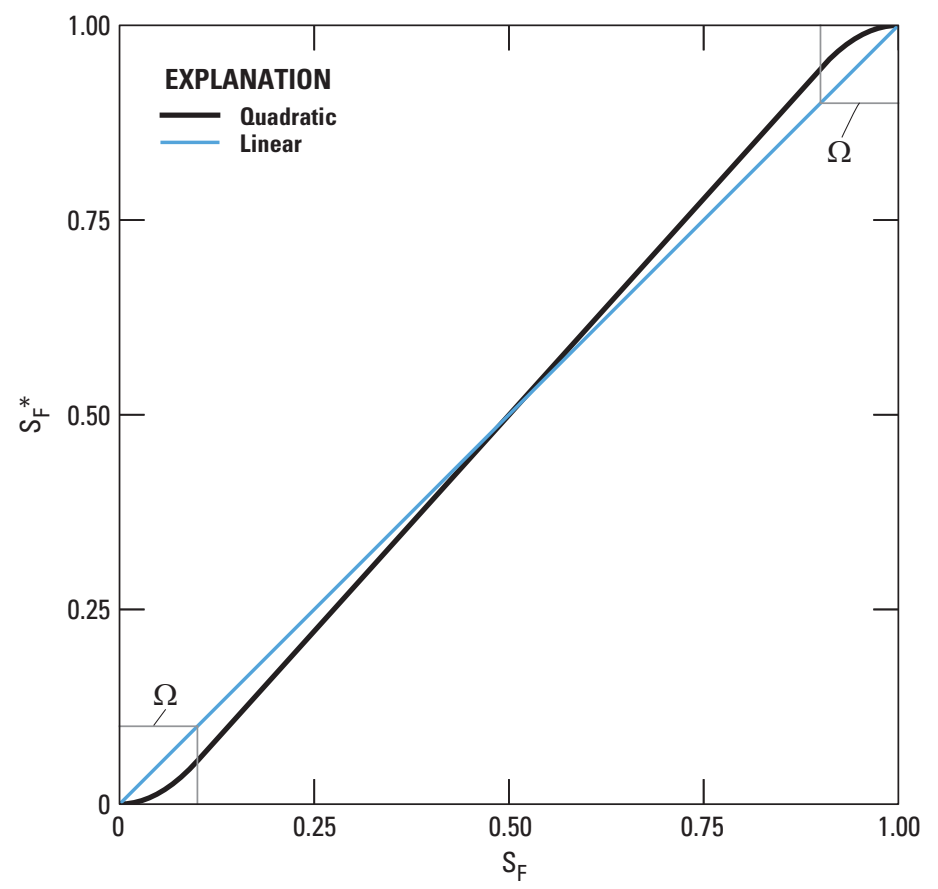

Figure 4-1. Graph showing combined quadratic and linear functions used to smooth the saturated fraction for a cell. $S_{F}$ is saturated fraction $\left(\frac{h-B O T}{T O P-B O T}\right)$ and $S_{F}{ }^{*}$ is the smoothed saturated fraction. Figure modified from Niswonger and others, 2011.

$$
S_{F_{n}}^{*}= \begin{cases}0 & \text { for } S_{F_{n}}<0 \\ \frac{A_{\Omega}}{2 \Omega} S_{F_{n}}{ }^{2} & \text { for } 0 \leq S_{F_{n}}<\Omega \\ A_{\Omega} S_{F_{n}}+\frac{1}{2}\left(1-A_{\Omega}\right) & \text { for } \Omega \leq S_{F}<(1-\Omega) \\ 1-\frac{A_{\Omega}}{2 \Omega}\left(1-S_{F_{n}}\right)^{2} & \text { for }(1-\Omega) \leq S_{F_{n}}<1 \\ 1 & \text { for } S_{F_{n}} \geq 1\end{cases}
$$

where,

$$
S_{F_{n}}=\frac{\Delta v_{n}}{T O P_{n}-B O T_{n}}
$$

$\Omega$ is a small distance over which the smoothing occurs $(L)$ and $A_{\Omega}$ is defined as $A_{\Omega}=\frac{1}{1-\Omega}$. As indicated by equations 4-5 and 4-6, the saturated fraction will be 1 for confined cells and for convertible cells in which the head is above the cell top elevation (fig. 4-1). As described by Niswonger and others (2011), the value for $\Omega$ should be set to a small value. In MODFLOW 6, this value is fixed at $1 \times 10^{-6}$.

In MODFLOW 6 , the equation for calculating the saturated thickness of cell $n, \Delta v_{n}$, is

$$
\Delta v_{n}=S_{F_{n}}\left(T O P_{n}-B O T_{n}\right)
$$




\section{4-4 Documentation for the MODFLOW 6 Groundwater Flow Model}

$\boldsymbol{A}$

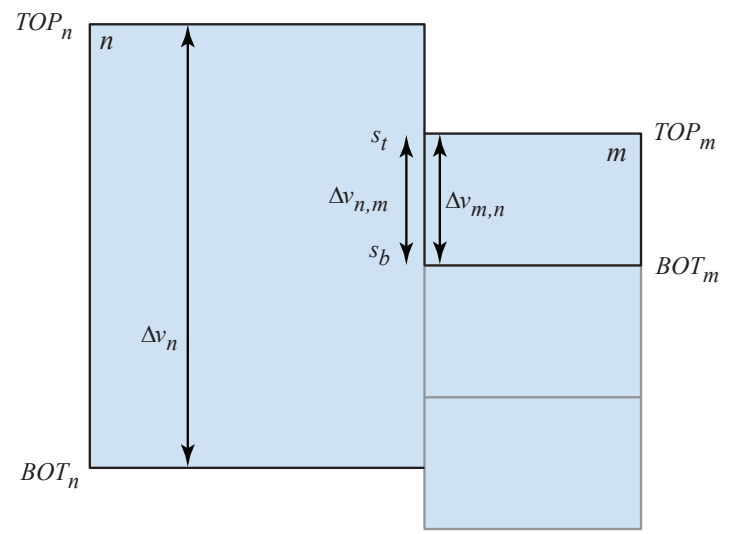

B

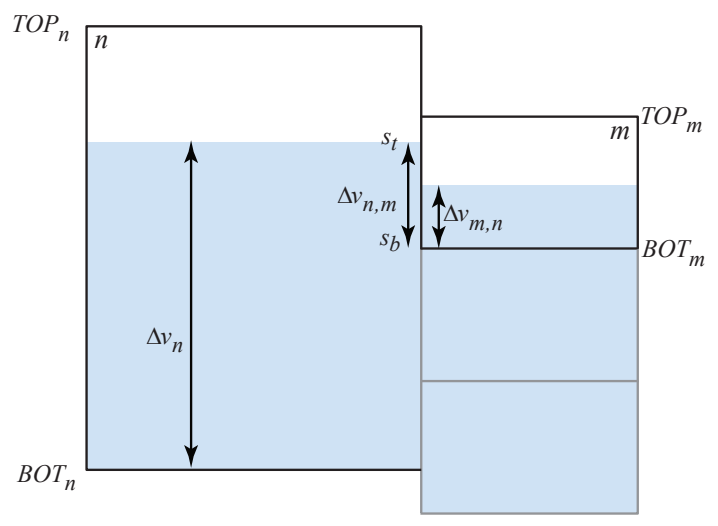

Figure 4-2. Diagram of a cross section showing cell $n$ connected to vertically staggered cells, including cell $m$. $A$, confined conditions, and $B$, for unconfined conditions.

or,

$$
\Delta v_{n}=S_{F_{n}}^{*}\left(T O P_{n}-B O T_{n}\right),
$$

if the Newton-Raphson formulation is used.

\section{Vertically Staggered Grid}

As described in chapter 3 for the DISU Package, horizontal connections can be marked as being vertically staggered. Horizontal connections for the GWF Exchanges can also be marked as vertically staggered. For a vertically staggered grid, such as the ones shown in figure 4-2, cell $n$ may be connected to more than one cell in any horizontal direction. In this case, the saturated thickness (and thus, the transmissivity value used in averaging equations below) for cell $n$ is calculated differently for each horizontal connection. To denote that the saturated thickness for cell $n$ depends on the connection, another subscript is added; $\Delta v_{n, m}$ denotes the saturated thickness for cell $n$ in the direction of cell $m$. For confined conditions, $\Delta v_{n, m}$ is equal to $\Delta v_{m, n}$ and is calculated as

$$
\Delta v_{n, m}=s_{t}-s_{b},
$$

where $s_{t}$ is the minimum top elevation $(L)$, defined as

$$
s_{t}=\min \left(T O P_{n}, T O P_{m}\right)
$$

and $s_{b}$ is the maximum bottom elevation $(L)$, defined as

$$
s_{b}=\max \left(B O T_{n}, B O T_{m}\right) .
$$

For unconfined conditions, $\Delta v_{n, m}$ is calculated by considering the position of the water table in the cell and the minimum top and maximum bottom elevations for the connection between cells $n$ and $m$. 


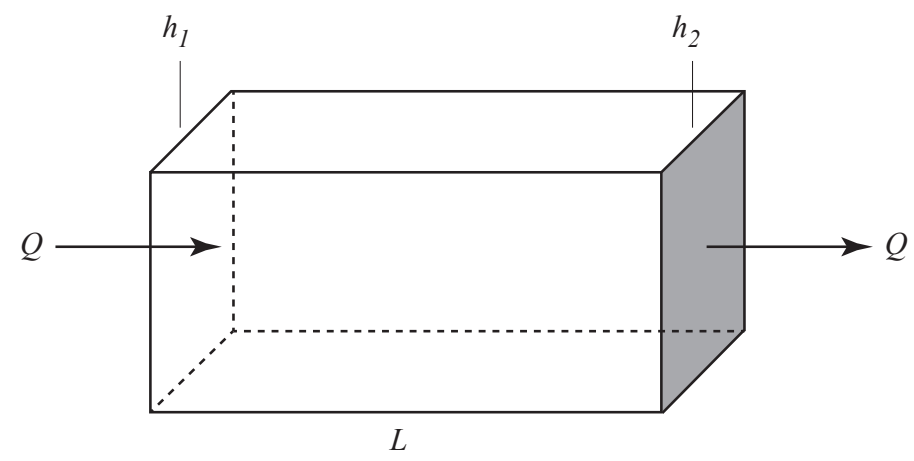

Figure 4-3. Diagram of prism of porous material used to illustrate Darcy's Law. Figure modified from McDonald and Harbaugh (1988).

$$
\Delta v_{n, m}= \begin{cases}s_{t}-s_{b} & \text { if } B O T_{n}+\Delta v_{n} \geq s_{t} \\ \Delta v_{n}-s_{b}+B O T_{n} & \text { if } B O T_{n}+\Delta v_{n}<s_{t}\end{cases}
$$

For internal flows within a single GWF Model, there is only one case where these vertically staggered connections apply - when the DISU Package is used to define the grid and when the connection itself is marked as a vertically staggered connection by specifying an $I H C$ value of 2 . Vertically staggered connections can also be specified when two GWF Models are connected with a GWF Exchange.

\section{Basic Hydraulic Conductance Equations}

The concept of hydraulic conductance (shortened to conductance in this report) is defined in chapter 2. Conductance is reviewed here, including the calculation of equivalent conductance for prismatic elements arranged in series. Conductance is a combination of several parameters used in Darcy's Law. Darcy's Law defines one-dimensional flow in a prism of porous material (fig. 4-3) as

$$
Q=\frac{K A\left(h_{1}-h_{2}\right)}{L_{1,2}}
$$

where $Q$ is the volumetric flow $\left(L^{3} T^{-1}\right), K$ is the hydraulic conductivity of the material in the direction of flow $\left(L T^{-1}\right), A$ is the cross-sectional area perpendicular to flow $\left(L^{2}\right)$, and $L_{1,2}$ is the length of the prism parallel to the flow path between $h_{1}$ and $h_{2}(L)$.

Conductance, $C$, is defined as

$$
C=\frac{K A}{L_{1,2}}
$$

Therefore, Darcy's Law can be written as

$$
Q=C\left(h_{1}-h_{2}\right) \text {. }
$$




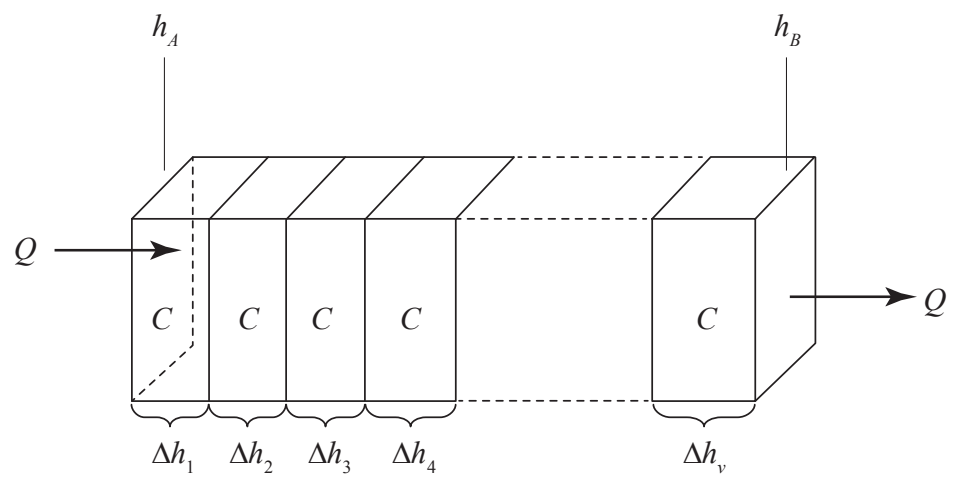

Figure 4-4. Diagram showing several prisms in series. Schematic is used to show relation between flow and head loss through several prisms in series. Figure modified from McDonald and Harbaugh (1988).

Another form of the conductance definition for horizontal flow in a prism is

$$
C=\frac{T W}{L_{1,2}}
$$

where $T$ is transmissivity ( $K$ times thickness of the prism) in the direction of flow $\left(L^{2} T^{-1}\right)$; and $W$ is the width of the prism $(L)$.

Conductance is defined for a particular prism of material and for a particular direction of flow. In an anisotropic medium that is characterized by three principal directions of hydraulic conductivity, the conductances of a prism in these three principal directions will generally differ.

If a prism of porous material consists of two or more subprisms in series (aligned sequentially in the direction of one-dimensional flow) as shown in figure 4-4, and the conductance of each subprism is known, a conductance representing the entire prism can be calculated. The equivalent conductance for the entire prism is the rate of flow in the prism divided by the head change across the prism, as shown in the following equation:

$$
C=\frac{Q}{\left(h_{A}-h_{B}\right)}
$$

Assuming continuity of head across each subprism gives the identity

$$
\sum_{i=1}^{n} \Delta h_{i}=h_{A}-h_{B}
$$

and substituting for head change across each subprism using Darcy's Law (eq. 4-15) gives

$$
\sum_{i=1}^{n} \frac{q_{i}}{c_{i}}=h_{A}-h_{B}
$$

where $q_{i}$ is the flow across subprism $i$, and $c_{i}$ is the conductance of subprism $i$. 
Because flow is one dimensional, and we assume no accumulation or depletion in storage, each $q_{i}$ is equal to the total flow $Q$; therefore,

$$
Q \sum_{i=1}^{n} \frac{1}{c_{i}}=h_{A}-h_{B}
$$

and

$$
\frac{h_{A}-h_{B}}{Q}=\sum_{i=1}^{n} \frac{1}{c_{i}} .
$$

Equating equations 4-21 and 4-17 results in

$$
\frac{1}{C}=\sum_{i=1}^{n} \frac{1}{c_{i}}
$$

Thus, for a set of conductances arranged in series, the inverse of the equivalent conductance equals the sum of the inverses of the individual conductances. When only two subprisms exist, the equivalent conductance reduces to

$$
C=\frac{c_{1} c_{2}}{c_{1}+c_{2}}
$$

\section{Standard Formulation-Horizontal Conductance}

The CVFD equations in MODFLOW 6 use equivalent conductances between nodes of adjacent cellsthat is, "branch conductances"-rather than conductances defined within individual cells. For cells that are connected horizontally, the $C_{n, m}$ term in equation 4-2 represents the horizontal conductance. The NPF Package reads data defining the horizontal hydraulic conductivity for individual cells and calculates the branch conductance between cells. Four methods of calculating these conductances are supported. The first method is based on the original MODFLOW approach (McDonald and Harbaugh, 1988). The next two methods are described by Goode and Appel (1992). The last method is described in Painter and others (2008) and implemented in MODFLOW-USG (Panday and others, 2013). The methods differ in the assumptions about the way the groundwater system varies from cell to cell. The methods are as follows:

- "harmonic-mean" method (assumes transmissivity is constant within a cell);

- "logarithmic-mean" method (assumes transmissivity varies linearly between nodes);

- "arithmetic-mean thickness and logarithmic-mean hydraulic conductivity" method of computing transmissivity (assumes the aquifer is flat with a water table in which hydraulic conductivity varies linearly between nodes); and

- "arithmetic-mean thickness and harmonic-mean hydraulic conductivity" method of computing transmissivity.

Previous MODFLOW versions included an "arithmetic-mean" method as part of the Block-Centered Flow (BCF) Package; however, this option is no longer supported as it reduces to the "arithmetic-mean thickness 


\section{4-8 Documentation for the MODFLOW 6 Groundwater Flow Model}

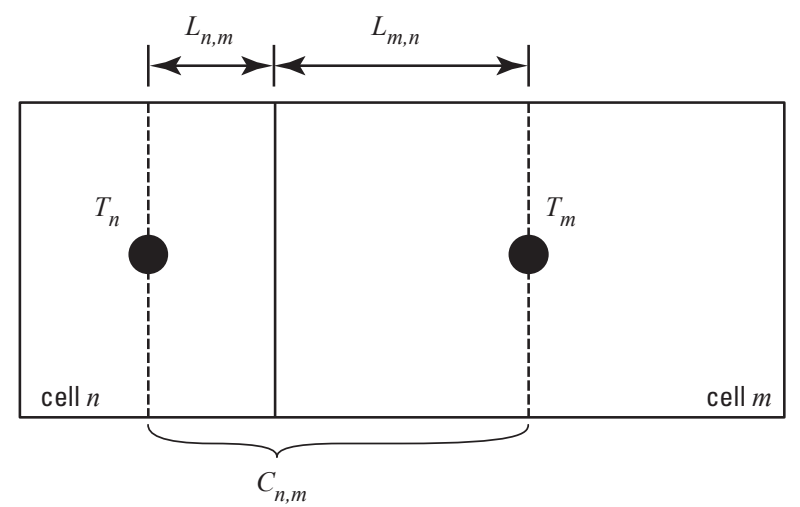

Figure 4-5. Diagram showing calculation of conductance between nodes using transmissivities and dimensions of cells. Figure modified from McDonald and Harbaugh (1988).

and logarithmic-mean hydraulic conductivity" method for homogeneous conditions. The supported four methods are described below.

The first method for computing horizontal conductance is based on the assumption that hydraulic conductivity times thickness (that is, transmissivity) is uniform within a cell. There can, however, be a discrete change in transmissivity at the boundary between any two cells. By use of this assumption and the previously mentioned assumption that nodes are in the center of cells, the conductance between the nodes is the equivalent conductance of two half cells in series. Figure 4-5 illustrates two adjacent cells based on these assumptions. Substituting the conductance for each half cell (eq. 4-16) into eq. 4-23 results in

$$
C_{n, m}=\frac{\frac{T_{n, m} \Delta w_{n, m}}{L_{n, m}} \frac{T_{m, n} \Delta w_{n, m}}{L_{m, n}}}{\frac{T_{n, m} \Delta w_{n, m}}{L_{n, m}}+\frac{T_{m, n} \Delta w_{n, m}}{L_{m, n}}},
$$

where $T_{n, m}$ is transmissivity in cell $n$ in the direction of cell $m\left(L^{2} T^{-1}\right), T_{m, n}$ is transmissivity in cell $m$ in the direction of cell $n\left(L^{2} T^{-1}\right), L_{n, m}$ is the distance between node $n$ and the shared face with node $m(L)$, and $L_{m, n}$ is the distance between node $m$ and the shared face with node $n(L)$.

Simplification of equation 4-24 results in

$$
C_{n, m}=\Delta w_{n, m} \frac{T_{n, m} T_{m, n}}{T_{n, m} L_{m, n}+T_{m, n} L_{n, m}}
$$

Equation 4-25 is used unless the transmissivity of either cell is zero. If the transmissivity of either cell is zero, then the conductance between the nodes is set equal to zero without evaluating equation 4-24. This avoids the possibility of unnecessarily evaluating equation 4-24 and possibly dividing by zero.

The above approach for calculating branch conductance is called the "harmonic mean" method. The reason for this name can be seen by manipulating equation $4-25$ into

$$
C_{n, m}=\left(\frac{L_{n, m}+L_{m, n}}{\frac{L_{n, m}}{T_{n, m}}+\frac{L_{m, n}}{T_{m, n}}}\right) \frac{\Delta w_{n, m}}{L_{n, m}+L_{m, n}} .
$$


The term in parentheses is the distance weighted harmonic mean of transmissivity of the two half cells $\bar{T}_{n, m}$, which by comparison with equation 4-16, can be seen to be the equivalent transmissivity between nodes $n$ and $m$.

In order to apply the harmonic mean computation (eq. 4-25) for horizontal conductance between confined cells, transmissivity for each cell must be computed from input. Transmissivity for cell $n$ in the direction of cell $m$ is computed as

$$
T_{n, m}= \begin{cases}\Delta v_{n} K_{n, m} & \text { if horizontal connection } \\ \Delta v_{n, m} K_{n, m} & \text { if vertically staggered horizontal connection }\end{cases}
$$

where $K_{n, m}$ is the hydraulic conductivity of cell $n$ in the direction of cell $m$. The "direction of cell $m$ " is explicitly mentioned here to allow for the presence of anisotropy, which is discussed later in this chapter.

For water-table conditions, transmissivity values $\left(T_{n, m}\right)$ are recalculated at the start of each iteration using equation 4-27 with an updated value for $\Delta v_{n}$ calculated using head from the previous iteration. Conductance is also recalculated using updated transmissivities, using equation 4-25. If head drops below the cell bottom (eq. 4-4), the cell is considered to be fully dewatered and is set to no flow, unless the Newton-Raphson formulation is used.

When transmissivity varies linearly between nodes, the interblock transmissivity derived by Goode and Appel (1992) is

$$
\bar{T}_{n, m}=\frac{T_{m, n}-T_{n, m}}{\ln \left(T_{m, n} / T_{n, m}\right)},
$$

where $\bar{T}$ represents the average transmissivity between horizontally adjacent cells $n$ and $m$ with linearly varying hydraulic properties $\left(L^{2} T^{-1}\right)$. Transmissivity values for the cells are computed using equation 4-27. This is called the "logarithmic-mean" interblock transmissivity method. This method produces exact solutions for uniform, steady-state, one-dimensional flow when the transmissivity varies in the direction of flow. To save numerical effort, this function is approximated by

$$
\bar{T}_{n, m}=\frac{T_{n, m}+T_{m, n}}{2} \text {, when } 0.995 \leq \frac{T_{m, n}}{T_{n, m}} \leq 1.005 .
$$

If either $T_{n, m}$ or $T_{m, n}$ is zero, then $\bar{T}_{n, m}$ is set to zero (instead of evaluating eq. 4-28 or 4-29) to avoid taking the logarithm of zero or dividing by zero.

For an unconfined aquifer with a flat bottom and with hydraulic conductivity varying linearly between nodes, Goode and Appel (1992) derive the interblock transmissivity as

$$
\bar{T}_{n, m}=\left(\frac{\Delta v_{n}+\Delta v_{m}}{2}\right)\left(\frac{K_{m, n}-K_{n, m}}{\ln \left(K_{m, n} / K_{n, m}\right)}\right) .
$$

This is called the "arithmetic-mean thickness and logarithmic-mean hydraulic conductivity" method. This method produces exact solutions for uniform, steady-state, one-dimensional flow when the hydraulic conductivity varies in the direction of flow. To save numerical effort, the hydraulic conductivity part of this function is approximated by $\frac{K_{n, m}+K_{m, n}}{2}$ when $0.995 \leq \frac{K_{m, n}}{K_{n, m}} \leq 1.005$. If either $K_{n, m}$ or $K_{m, n}$ is zero, then $\bar{T}_{n, m}$ is set to zero (instead of evaluating eq. 4-30) to avoid taking the logarithm of zero or dividing by zero. 


\section{4-10 Documentation for the MODFLOW 6 Groundwater Flow Model}

When either the "logarithmic-mean" or the "arithmetic-mean thickness and logarithmic-mean hydraulic conductivity" methods are used, $\bar{T}_{n, m}$ is substituted into the following equation to calculate the conductance between cells $n$ and $m$ :

$$
C_{n, m}=\frac{\bar{T}_{n, m} \Delta w_{n, m}}{L_{n, m}+L_{m, n}}
$$

In a description of the Newton-Raphson method for MODFLOW, Painter and others (2008) used an "arithmetic-mean thickness and harmonic-mean hydraulic conductivity" method to calculate the conductance between two cells. This method is also implemented in MODFLOW-USG for the Newton-Raphson formulation. The method is included in MODFLOW 6 for experimentation purposes and so that results from MODFLOW 6 can be compared with results from other models. The equation for conductance using this method is

$$
C_{n, m}=\left(\frac{K_{n, m} K_{m, n}}{L_{n, m} K_{m, n}+L_{m, n} K_{n, m}}\right) \Delta w_{n, m}\left(\frac{\Delta v_{n}+\Delta v_{m}}{2}\right)
$$

\section{Newton-Raphson Formulation-Horizontal Conductance}

The Newton-Raphson formulation can be used to solve for groundwater flow in aquifers that have thin saturated thicknesses and may transition between wet and dry. The Newton-Raphson formulation uses a fully saturated horizontal conductance, $C_{n, m}^{0}$, between cells $n$ and $m$. This fully saturated horizontal conductance is multiplied by the saturated fraction of the upstream cell. Calculation of $C_{n, m}^{0}$ is made by using the full cell thicknesses of $n$ and $m$ with one of the conductance weighting equations presented in the previous section. Values for $C_{n, m}^{0}$ are calculated once at the start of the simulation and do not need to be recalculated during the simulation as they do not vary as a function of head.

With the Newton-Raphson formulation, the flow term $Q_{n, m}$ between cell $n$ and neighbor $m$ is expressed as the product of the upstream saturated fraction, the saturated conductance, and the head difference, as

$$
Q_{n, m}=S_{F, m}^{*} C_{n, m}^{0}\left(h_{m}-h_{n}\right),
$$

where $S_{F n, m}^{*}$ is the upstream saturated fraction (eq. 4-5) calculated for cells $n$ and $m$ using equation 4-6 with $S_{F}$ as a function of $\Delta v_{u}$. The upstream saturated thickness $\Delta v_{u}$ is determined for the connection between cell $n$ and $m$ using

$$
\Delta v_{u}= \begin{cases}\Delta v_{n} & \text { if } h_{n} \geq h_{m} \\ \Delta v_{m} & \text { if } h_{n}<h_{m}\end{cases}
$$

The derivatives with respect to $h_{n}$ and $h_{m}$ are obtained by applying the chain rule to equation 4-33, which results in the following derivative expressions:

$$
\frac{\partial Q_{n, m}}{\partial h_{n}}=-S_{F n, m}^{*} C_{n, m}^{0}+\frac{\partial S_{F n, m}^{*}}{\partial h_{n}} C_{n, m}^{0}\left(h_{m}-h_{n}\right),
$$


and

$$
\frac{\partial Q_{n, m}}{\partial h_{m}}=S_{F n, m}^{*} C_{n, m}^{0}+\frac{\partial S_{F n, m}^{*}}{\partial h_{n}} C_{n, m}^{0}\left(h_{m}-h_{n}\right) .
$$

Note for upstream weighting that

$$
\frac{\partial S_{F n, m}^{*}}{\partial h_{n}}= \begin{cases}\frac{\partial S_{F n}^{*}}{\partial h_{n}} & \text { if } n \text { is upstream } \\ 0 & \text { if } m \text { is upstream }\end{cases}
$$

and

$$
\frac{\partial S_{F n, m}^{*}}{\partial h_{m}}= \begin{cases}\frac{\partial S_{F m}^{*}}{\partial h_{m}} & \text { if } m \text { is upstream } \\ 0 & \text { if } n \text { is upstream }\end{cases}
$$

The fully implicit form of the Newton-Raphson formulation for horizontal flow between cells $n$ and $m$ in the form of equation $2-30$ is

$$
\frac{\partial Q_{n, m}}{\partial h_{n}} h_{n}^{k}+\frac{\partial Q_{n, m}}{\partial h_{m}} h_{m}^{k}=-Q_{n, m}^{k-1}+\frac{\partial Q_{n, m}}{\partial h_{n}} h_{n}^{k-1}+\frac{\partial Q_{n, m}}{\partial h_{m}} h_{m}^{k-1} .
$$

Substitution of equations 4-33, 4-35, and 4-36 into equation 4-39 results in the following general expansion of the Newton-Raphson formulation for horizontal flow between cells $n$ and $m$ :

$$
\begin{gathered}
{\left[-S_{F n, m}^{*} C_{n, m}^{0}+\frac{\partial S_{F n, m}^{*}}{\partial h_{n}} C_{n, m}^{0}\left(h_{m}^{k-1}-h_{n}^{k-1}\right)\right] h_{n}^{k}+} \\
{\left[S_{F n, m}^{*} C_{n, m}^{0}+\frac{\partial S_{F n, m}^{*}}{\partial h_{n}} C_{n, m}^{0}\left(h_{m}^{k-1}-h_{n}^{k-1}\right)\right] h_{m}^{k}=} \\
{\left[-\left[S_{F n, m}^{*} C_{n, m}^{0}\left(h_{m}^{k-1}-h_{n}^{k-1}\right)\right]+\right.} \\
\left.-S_{F n, m}^{*} C_{n, m}^{0}+\frac{\partial S_{F n, m}^{*}}{\partial h_{n}} C_{n, m}^{0}\left(h_{m}^{k-1}-h_{n}^{k-1}\right)\right] h_{n}^{k-1}+ \\
{\left[S_{F n, m}^{*} C_{n, m}^{0}+\frac{\partial S_{F n, m}^{*}}{\partial h_{n}} C_{n, m}^{0}\left(h_{m}^{k-1}-h_{n}^{k-1}\right)\right] h_{m}^{k-1} .}
\end{gathered}
$$




\section{4-12 Documentation for the MODFLOW 6 Groundwater Flow Model}

Simplification of equation $4-40$ results in

$$
\begin{array}{r}
{\left[-S_{F n, m}^{*} C_{n, m}^{0}+\frac{\partial S_{F n, m}^{*}}{\partial h_{n}} C_{n, m}^{0}\left(h_{m}^{k-1}-h_{n}^{k-1}\right)\right] h_{n}^{k}+} \\
{\left[S_{F n, m}^{*} C_{n, m}^{0}+\frac{\partial S_{F n, m}^{*}}{\partial h_{n}} C_{n, m}^{0}\left(h_{m}^{k-1}-h_{n}^{k-1}\right)\right] h_{m}^{k}=} \\
\\
{\left[\frac{\partial S_{F n, m}^{*}}{\partial h_{n}} C_{n, m}^{0}\left(h_{m}^{k-1}-h_{n}^{k-1}\right)\right] h_{n}^{k-1}+} \\
{\left[\frac{\partial S_{F n, m}^{*}}{\partial h_{n}} C_{n, m}^{0}\left(h_{m}^{k-1}-h_{n}^{k-1}\right)\right] h_{m}^{k-1} .}
\end{array}
$$

The $S_{F_{n, m}}^{*} C_{n, m}^{0}$ terms in equation 4-41 are subtracted from the upstream diagonal and added to the appropriate off-diagonal terms of the coefficient matrix during the standard formulation, respectively. The NewtonRaphson formulation is completed by augmenting the coefficient matrix with the second term on the righthand side of equations 4-35 and 4-36, and adding the product of the second term on the right-hand side of equations 4-35 or 4-36 and the current upstream head to the right-hand side.

\section{Standard Formulation-Vertical Conductance}

Calculation of vertical conductance is required when cells are connected vertically. The default behavior for MODFLOW 6 is to calculate vertical conductance under the assumption that both cells $n$ and $m$ are fully saturated. This is the default behavior even if either of the cells are partially dewatered and the cells are marked as being convertible. This is also the default behavior for the Newton-Raphson formulation. The equation for vertical conductance when both cells are assumed to be fully saturated is

$$
\frac{1}{C_{n, m}}=\frac{1}{\frac{A_{n, m} K_{n, m}}{(1 / 2)\left(T O P_{n}-B O T_{n}\right)}}+\frac{1}{\frac{A_{n, m} K_{m, n}}{(1 / 2)\left(T O P_{m}-B O T_{m}\right)}},
$$

where $A_{n, m}$ is the flow area of the connection (as viewed from above) between cells $n$ and $m\left(L^{2}\right)$. Experience suggests that equation 4-42 is the most stable equation for vertical conductance and should be used in most cases. Equation 4-42 is consistent with the horizontal conductance equations for flow in series (eqns. 4-24 and 4-25), where the distances are the vertical distances between nodes.

The NPF Package has several options that can be used to change the way the vertical conductance is calculated. These options can affect simulation results when a partially saturated convertible cell underlies a saturated or partially saturated overlying cell as shown in figure 4-6. These options may improve the accuracy of the solution for perched aquifer conditions and for aquifer drainage problems in which the water table rises and falls over multiple model layers.

MODFLOW 6 has an option to recalculate the vertical conductance each time the head solution is updated. This option is activated with the "VARIABLECV" keyword in the NPF Package. When the "VARIABLECV" option is activated, vertical conductance is calculated using the following (see fig. 4-6 for explanation of variables):

$$
\frac{1}{C_{n, m}}=\frac{1}{\frac{A_{n, m} K_{n, m}}{(1 / 2) \Delta v_{n}}}+\frac{1}{\frac{A_{n, m} K_{m, n}}{(1 / 2)\left(T O P_{m}-B O T_{m}\right)}} .
$$




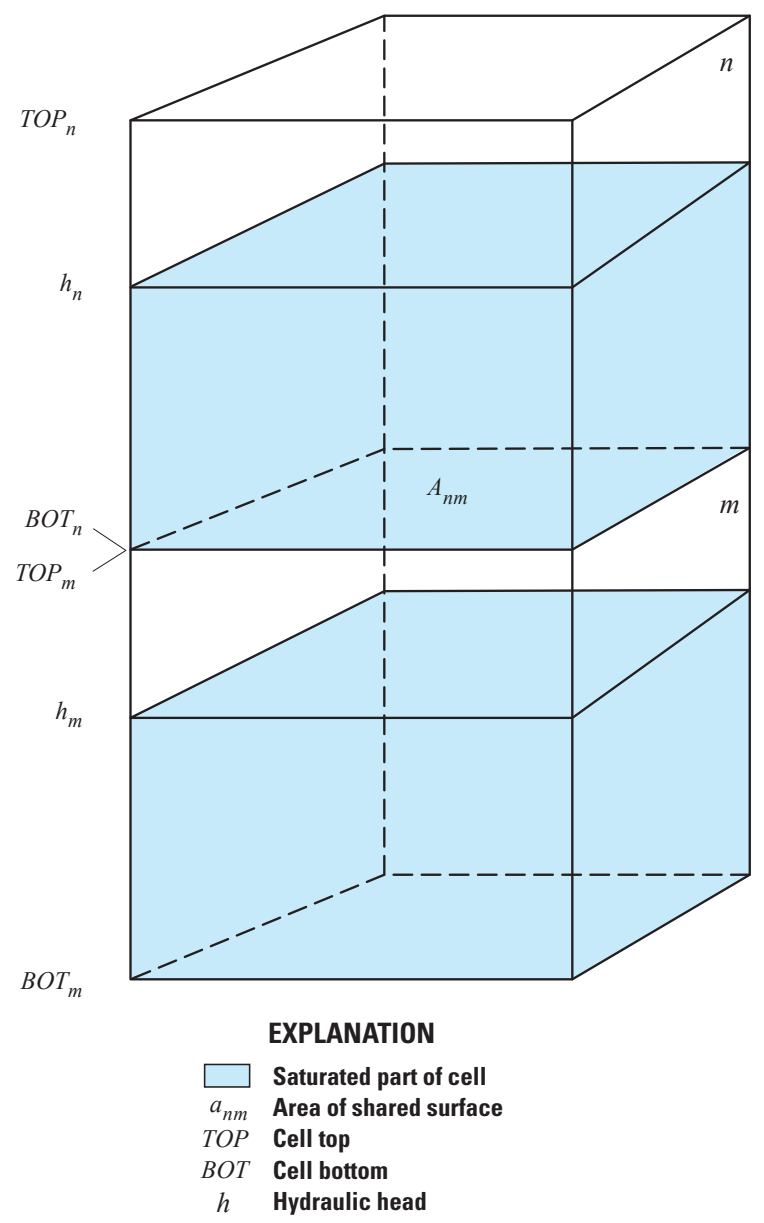

Figure 4-6. Diagram showing the situation in which a correction is required to limit the downward flow from cell $n$ into $m$, as a result of cell $m$ being partially dewatered. Figure modified from Panday and others (2013). 


\section{4-14 Documentation for the MODFLOW 6 Groundwater Flow Model}

This equation for vertical conductances is the same as equation 4-42 when cell $n$ is marked as a confined cell or when it is fully saturated. When the "VARIABLECV" option is activated, the simulation may become less stable than for the default condition; however, simulation results may be more accurate for drainage problems in which the water tables rises and falls over multiple model layers.

The "VARIABLECV" option can also be supplemented with the "DEWATERED" option. This may further improve the accuracy of simulation results for drainage problems. In this case, the vertical conductance is calculated as

$$
\frac{1}{C_{n, m}}=\frac{1}{\frac{A_{n, m} K_{n, m}}{(1 / 2) \Delta v_{n}}} .
$$

This equation is different from equation $4-43$ in that the vertical resistance to flow for cell $m$ is not included in the calculation of vertical conductance. This is the least stable equation for vertical conductance because there can be an abrupt change in vertical conductance whenever cell $m$ converts between fully saturated and partially dewatered.

The NPF Package has a "PERCHED" option that can be activated to approximate perched water-table conditions. When this capability is activated, the vertical flow calculation is modified if a cell is convertible, its head is below its top elevation, and the cell directly above is fully or partially saturated (fig. 4-6, for example). Such a situation can occur, for example, under perched conditions. In this case, the "PERCHED" option can be conceptualized as using the weight of the overlying water column to drive flow. The following paragraphs describe implementation of this capability.

The expression (eq. 2-5) for flow between cell $n$ and $m$ is

$$
Q_{n, m}=C_{n, m}\left(h_{m}-h_{n}\right)
$$

Following the convention of MODFLOW, a positive value of $Q_{n, m}$ indicates flow into cell $n$ and a negative value indicates flow out of the cell $n$. Equation 4-45 is based on the assumption that cells $n$ and $m$ are fully saturated - that is, the hydraulic head in each cell stands higher than the elevation of the top of the cell.

Situations can occur, however, in which part of a confined aquifer may become unsaturated: for example, when drawdown due to pumpage causes water levels to fall, at least locally, below the top of an aquifer. This condition is most likely to occur when an aquifer is overlain by a lower conductivity unit. In terms of simulation, this condition is shown in figure 4-6. Cells in two model layers are represented. The upper cell $n$ has lower hydraulic conductivity than the underlying cell $m$, which represents an aquifer. Pumping from the lower layer has lowered the water level in cell $m$ below the elevation of the top of the cell; however, cell $n$ remains fully or partially saturated. Thus, cell $m$ is effectively unconfined even though the cell above is saturated.

Consider the calculation of flow between nodes in the upper and lower cells. In the upper cell, head is simply whatever head is at that cell- $h_{n}$. Just below the upper cell, however, unsaturated conditions prevail, so that the pressure sensed on the lower surface of the confining unit is atmospheric - taken as zero in the model formulation. Thus, the head at the bottom of the upper cell is simply the elevation at that point- that is, the elevation of the top of the lower cell. If this elevation is designated $B O T_{n}$, then the actual flow through the confining bed is obtained by substituting $B O T_{n}$ for $h_{m}$ in equation $4-45$, which results in

$$
Q_{n, m}=C_{n, m}\left(B O T_{n}-h_{n}\right)
$$

Thus, the flow will be downward, from cell $n$ to cell $m$, but under this condition the flow will no longer be dependent on the water level, $h_{m}$, in the lower cell. The simplest approach to this problem in formulating 
the equation for cell $n$ is to substitute the flow expression of equation 4-46 into equation 4-1, in place of the expression given in equation 4-45. A consequence of this substitution is that the matrix of coefficients of the finite-difference equations becomes asymmetric. Most solvers available in previous MODFLOW versions were not designed for asymmetric matrices; thus, previous MODFLOW versions used a correction approach, instead of the direct substitution approach used in MODFLOW 6, to solve for perched conditions.

For many groundwater flow problems, perched aquifer conditions do not occur at the scale of interest or their representation is not important to simulation objectives. Moreover, for some aquifer drainage problems, perched conditions may occur numerically, when in fact the aquifer is fully saturated beneath the water table. For these reasons, the "PERCHED" option is no longer the default behavior in MODFLOW 6, as it was in previous versions, and users must intentionally activate use of equation 4-46. If the "PERCHED" option is activated, then it may be necessary to use a linear acceleration method that is designed for asymmetric matrices, such as the biconjugate gradient method. Use of a linear acceleration method designed for symmetric matrices (the conjugate gradient method, for example) may not converge or may not converge quickly if the PERCHED option is specified and perched conditions are present in the model. The VARIABLECV, DEWATERED, and PERCHED options are not recommended for most applications; they may be useful, however, for drainage and perched conditions or for examining differences in results between MODFLOW 6 and other MODFLOW versions.

\section{Newton-Raphson Formulation-Vertical Conductance}

The constant vertical conductance approach (eq. 4-42) is the default approach used when the NewtonRaphson formulation is used. Because vertical conductance is constant with this approach, the derivatives in the vertical direction are zero. As a result, the conductance terms in the coefficient matrix are not modified for vertical connections when the Newton-Raphson formulation is used.

\section{Calculation of Effective Hydraulic Conductivity}

As discussed in chapter 2, the CVFD equation can provide an accurate representation of flows between model cells if the grid satisfies certain geometric requirements and the conductivity is isotropic, or if the grid is regular and the principal directions of conductivity are aligned with the grid axes. Under either of those conditions, Darcy's Law can be expressed in the form of equation 2-1, and for each cell connection, it reduces to a scalar equation that relates the fluid flux to the head gradient along the connection

$$
q_{c}=-K \frac{\partial h}{\partial x_{c}}
$$

where $q_{c}$ is the fluid flux along the connection $\left(L T^{-1}\right)$ and $x_{c}$ represents distance along the connection $(L)$. The conductance-based flow expression then follows directly from the finite-difference discretization of equation 4-47. The preceding subsections described how the NPF Package calculates horizontal and vertical conductances for the conductance-based flow expression under the conditions that reduce Darcy's Law to equation 4-47.

In the most general case, Darcy's Law in its full, tensorial form is

$$
\mathbf{q}=-\mathbf{K} \nabla h=-\left(\begin{array}{lll}
K_{x x} & K_{x y} & K_{x z} \\
K_{x y} & K_{y y} & K_{y z} \\
K_{x z} & K_{y z} & K_{z z}
\end{array}\right) \nabla h .
$$




\section{4-16 Documentation for the MODFLOW 6 Groundwater Flow Model}

In that case, each component of the flow vector, $\mathbf{q}$, depends on all three components of the head-gradient vector, $\nabla h$, via an anisotropic conductivity tensor $\mathbf{K}$. Therefore, in the most general case, calculations of fluid flux should ideally be based on an estimate of the complete head-gradient vector. An approach that is rigorous in that regard was previously available in MODFLOW only in the LVDA Capability in the HUF Package (Anderman and others, 2002). The LVDA approach was limited to variable-direction anisotropy within the horizontal plane.

The NPF Package of MODFLOW 6 approximates 4-48 using the conductance-based flow expression and bases the conductance on an "effective conductivity" for the connection. Under the conditions that reduce Darcy's Law to equation 4-47, as discussed above, the effective conductivity is the true conductivity, and the conductance-based flow expression gives an accurate estimate of the flow along the connection. In the most general case, however, the effective-conductivity approach described here is a mathematical approximation that might not yield an accurate estimate of the flow along the connection.

The NPF Package calculates the effective conductivity for the connection from a "conductivity ellipsoid" (or, in two dimensions, a "conductivity ellipse"), which is a geometric representation of the conductivity tensor (Freeze and Cherry, 1979). For a three-dimensional conductivity tensor whose conductivities along the three principal directions are $K_{11}, K_{22}$, and $K_{33}$, the conductivity ellipsoid has principal semi-axes of length $\sqrt{K_{11}}$, $\sqrt{K_{22}}$, and $\sqrt{K_{33}}$. Users specify values for $K_{11}, K_{22}$, and $K_{33}$. Freeze and Cherry (1979) define a conductivity, which they call $K_{s}$, that relates the magnitude of the fluid flux to the component of the head gradient along the flow direction. They then show that the square root of $K_{s}$ is the radius, defined here as the distance from the center to a point on the conductivity ellipsoid, measured along the flow direction.

The approach used by the NPF Package is an ad hoc adaptation of the Freeze and Cherry (1979) definition in which the square root of the effective conductivity for the connection, $\sqrt{K_{n, m}}$, is the radius of the conductivity ellipsoid measured along the direction normal to the interface. Panday and others (2013) used a conceptually similar method in MODFLOW-USG based on effective conductivity, although it defined the "conductivity ellipse" differently and, like LVDA, was limited to variable-direction anisotropy only within the horizontal plane. On a grid that conforms to the standard CVFD requirements-for example, a regular grid - the direction normal to the interface coincides with the straight-line connection between the nodes.

Figure 4-7 illustrates the calculation of effective conductivity from the conductivity ellipse in two dimensions. For a regular MODFLOW grid specified with the DIS Package, the $x$ and $y$ directions correspond to the directions along rows and columns, respectively (fig. 4-7A). In the figure 4-7A example, there is no rotation of the conductivity ellipse relative to the grid, but different values are specified for $K_{11}$ and $K_{22}$ in both cells $n$ and $m$. This case can be handled accurately with the effective conductivity approach, because the grid is aligned with the principal axes of the ellipse. Previous MODFLOW versions and MODFLOW 6 can represent this case accurately. Figure 4-7B also shows a regular grid, but in this case, the conductivity ellipses are rotated and scaled differently within each cell. This figure illustrates the calculation of the effective conductivities, $K_{n, m}$ and $K_{m, n}$, for cells $n$ and $m$, respectively. Calculation of the $K_{n, m}$ and $K_{m, n}$ ellipse radii correspond to the direction that is normal to the shared $n-m$ face. Figure $4-7 C$ shows how $K_{n, m}$ and $K_{m, n}$ are calculated when the line connecting the cell centers does not bisect the shared face at a right angle. In this case, the radii are intentionally calculated along a direction that is normal to the shared face rather than being calculated along a direction between the cell centers. Lastly, figure 4-7D shows how the effective conductivity calculation is made for nonrectangular cells with a rotated hydraulic conductivity ellipse. Here again, $K_{n, m}$ and $K_{m, n}$ are calculated in the direction normal to the shared face. In figures 4-7B, $C$, and $D$, the conductivity ellipses are rotated relative to the grid. Consequently, these representations are subject to errors inherent with this effective conductivity approach.

When the principal axes of conductivity are aligned with a regular model grid (for example, as in fig. 4$7 A$ ), the effective conductivity for a connection along a row is then the squared radius along the $x$ direction, or $K_{11}$, as one would expect. Similarly, the effective conductivity for a connection along a column is the squared radius along the $y$ direction, or $K_{22}$. When the principal axes of conductivity are not aligned with the model 
A
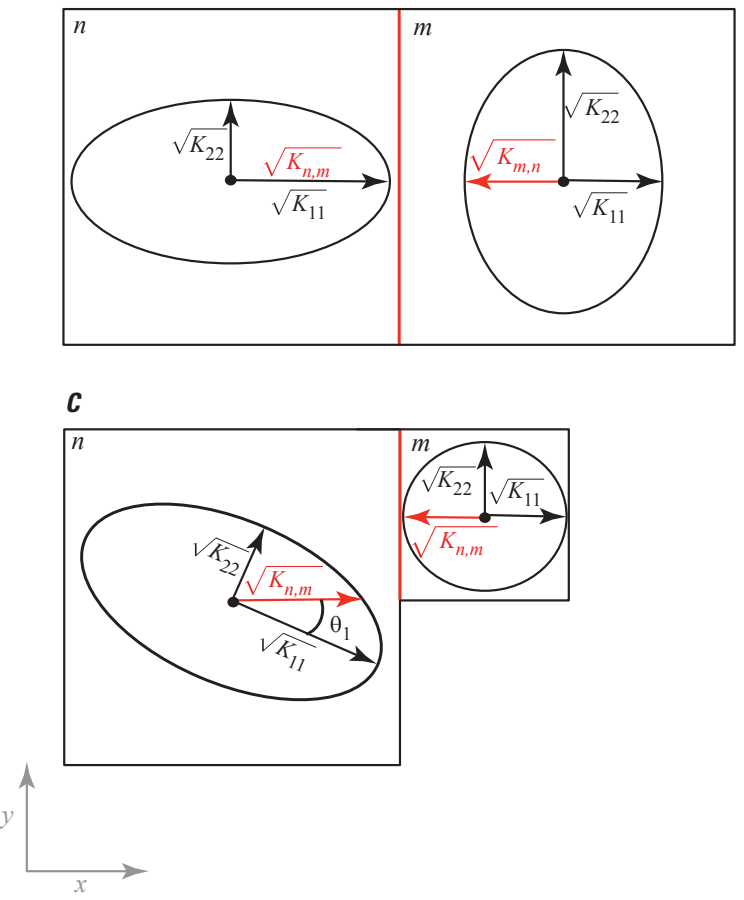

\section{B}

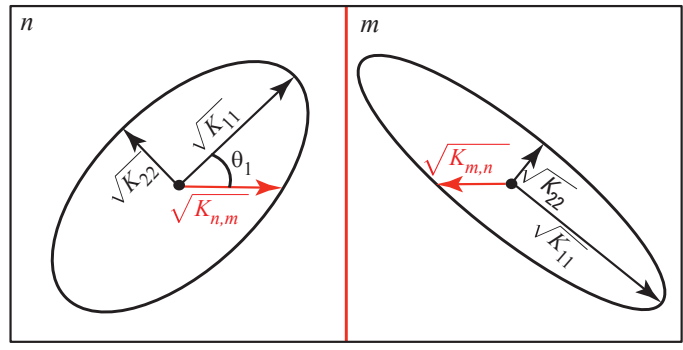

D

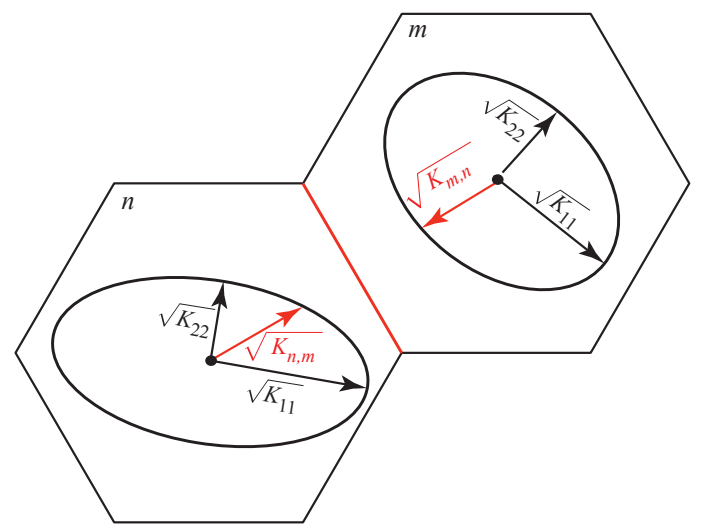

Figure 4-7. Diagram showing anisotropic hydraulic conductivity ellipses for several different configurations in plan view to illustrate the effective hydraulic conductivity calculation for a model cell $n$ connected to cell $m$. $A$, an unrotated ellipse case with a regular MODFLOW grid in which the principal axes of conductivity align with the grid; $B$, a rotated ellipse case with a regular MODFLOW grid in which the principal axes of conductivity do not align with the grid; $C$, a rotated ellipse case for a quadtree grid in which a line connecting the cell centers does not bisect the shared face at a right angle; and $D$, a rotated ellipse case for nonrectangular cells. The shared face is shown in red. 


\section{4-18 Documentation for the MODFLOW 6 Groundwater Flow Model}

grid, as in figure 4-7B, the principal conductivities $K_{11}$ and $K_{22}$ are no longer the effective conductivities along rows and columns. In that case, the radii of the ellipse in the $x$ and $y$ directions depend on the angle at which the principal directions of conductivity are rotated relative to the grid axes. For example, if the principal directions are rotated by a counterclockwise angle $\theta_{1}$ relative to the $x$ axis (as shown in fig. $4-7 B$ for cell $n$ ), then the effective conductivity along a row is the squared radius along the $x$ direction and is given by

$$
\frac{1}{K_{n, m}}=\frac{\cos ^{2} \theta_{1}}{K_{11}}+\frac{\sin ^{2} \theta_{1}}{K_{22}}
$$

Equation 4-49 can also be written as

$$
\frac{1}{K_{n, m}}=\frac{n_{K 1}^{2}}{K_{11}}+\frac{n_{K 2}^{2}}{K_{22}}
$$

where $n_{K 1}$ and $n_{K 2}$ are the components of the unit vector in the direction normal to the interface, $\mathbf{n}_{K}$, expressed in coordinates that are aligned with the $K_{11}$ and $K_{22}$ principal directions, respectively. In three dimensions, equation 4-50 generalizes to

$$
\frac{1}{K_{n, m}}=\frac{n_{K 1}^{2}}{K_{11}}+\frac{n_{K 2}^{2}}{K_{22}}+\frac{n_{K 3}^{2}}{K_{33}}
$$

where $n_{K 1}, n_{K 2}$, and $n_{K 3}$ are the components of the unit vector in the direction normal to the interface, $\mathbf{n}_{K}$, expressed in coordinates that are aligned with the $K_{11}, K_{22}$, and $K_{33}$ principal directions, respectively.

$K_{11}, K_{22}$, and $K_{33}$ values can be specified on a cell-by-cell basis so that the user has the option to vary the principal conductivities throughout the model grid. Care should be used when assigning these principal conductivities so that their orientations are consistent with the intended directions.

If the principal directions of conductivity do not align with the global model coordinates $x, y$, and $z$, then up to three rotation angles can be specified for each model cell. As shown in figure 4-7B for an ellipse, $\theta_{1}$ is the counterclockwise rotation of the conductivity ellipsoid within the $(x, y)$ plane. The rotation of the conductivity ellipsoid upward or downward from the $(x, y)$ plane is $\theta_{2}$. The rotation of the conductivity ellipsoid about the axis corresponding to $K_{11}$ is $\theta_{3}$. As described by Voss and Provost (2010), "These three angles may be thought of, in aeronautical terms, as the 'yaw,' 'pitch,' and 'roll' of the ... ellipsoid with respect to a reference orientation."

Given $\mathbf{n}_{g}$, the unit vector in the direction normal to the interface, expressed in global model coordinates, the NPF Package calculates the components of $\mathbf{n}_{K}$, which are used in equation 4-51 via a coordinate rotation, as shown in the following equation:

$$
\mathbf{n}_{K}=\mathbf{n}_{g} \mathbf{R}^{\mathbf{T}}
$$

where $\mathbf{R}$ is the rotation matrix

$$
\mathbf{R}=\left(\begin{array}{lll}
R_{1,1} & R_{1,2} & R_{1,3} \\
R_{2,1} & R_{2,2} & R_{2,3} \\
R_{3,1} & R_{3,2} & R_{3,3}
\end{array}\right)
$$


with elements

$$
\begin{aligned}
& R_{1,1}=\cos \theta_{1} \cos \theta_{2} \\
& R_{1,2}=\cos \theta_{1} \sin \theta_{2} \sin \theta_{3}-\sin \theta_{1} \cos \theta_{3} \\
& R_{1,3}=-\cos \theta_{1} \sin \theta_{2} \cos \theta_{3}-\sin \theta_{1} \sin \theta_{3} \\
& R_{2,1}=\sin \theta_{1} \cos \theta_{2} \\
& R_{2,2}=\sin \theta_{1} \sin \theta_{2} \sin \theta_{3}+\cos \theta_{1} \cos \theta_{3} \\
& R_{2,3}=-\sin \theta_{1} \sin \theta_{2} \cos \theta_{3}+\cos \theta_{1} \sin \theta_{3} \\
& R_{3,1}=\sin \theta_{2} \\
& R_{3,2}=-\cos \theta_{2} \sin \theta_{3} \\
& R_{3,3}=\cos \theta_{2} \cos \theta_{3} .
\end{aligned}
$$

\section{Cell Rewetting}

If the Newton-Raphson formulation is used, then cells are always considered active, and there is no reason to convert a dry cell to wet or a wet cell to dry. If the standard formulation is used, then the NPF Package can optionally be used to convert dry cells to wet using the approach described in this section. The drying and wetting approach described in this section is considered to be unstable for many applications. For this reason, the Newton-Raphson formulation is recommended for most applications that involve simulation of the water table.

When saturated thickness is zero as defined by head being less than the bottom elevation of a cell (eq. 4 4), it is clear that a variable-head (wet) cell should convert to dry. Unfortunately, there is not a straightforward way to know when a dry cell should convert to wet. McDonald and others (1992) describe an approach for doing this and its application in the BCF Package of an earlier version of MODFLOW. The same approach is applied in MODFLOW 6. The following is a brief overview of the approach. Readers should refer to McDonald and others (1992) for additional details. In particular, that report describes detailed information about numerical instabilities that can result from using this option, and several test problems are included.

A dry cell converts to wet based upon the head in an adjacent cell compared to a wetting threshold, THRESH, for the cell. If the head at the adjacent cell equals or exceeds the threshold at the start of a solution iteration, the dry cell is converted to wet. One option is to allow any of the cells that are directly adjacent to the cell horizontally, or any of the underlying cells, to cause a dry cell to convert to wet. Another option is to use only cells below to determine when a dry cell becomes wet. The head below can be a better wetting indicator than the head at horizontally adjacent cells when the head variations between adjacent horizontal cells are larger than the vertical head variations, which is frequently the case.

A primary difficulty with this approach is that the value of THRESH must be determined by trial and error. If $T H R E S H$ is too low, a cell may be incorrectly converted to wet. That is, a cell may convert to wet and then reconvert to dry in later solver iterations because the head does not stay above the bottom elevation. This cycle of converting between wet and dry conditions can repeat indefinitely, preventing the solver from converging. Larger values of THRESH can avoid repeated conversion between wet and dry, but they increase the nonuniqueness of the solution (McDonald and others, 1992, p. 8).

When a cell converts to wet, the initial estimate of head is established according to one of the two following equations:

$$
h_{n}=B O T_{n}+W E T F C T\left(h_{m}-B O T_{n}\right),
$$




\section{4-20 Documentation for the MODFLOW 6 Groundwater Flow Model}

or

$$
h_{n}=B O T_{n}+W E T F C T\left(T H R E S H_{n}\right),
$$

where $W E T F C T$ is a user-specified constant, generally between 0 and 1 , and $h_{m}$ is the head at the neighboring cell that causes cell $n$ to convert to wet. The user chooses the equation that works best for the problem being simulated. The initial estimate of head at a cell that converts to wet is theoretically unimportant because the solver should calculate the correct value in subsequent iterations. In practice, however, the initial estimate is often important for solver efficiency; that is, if a poor choice is made, the solver convergence may be slower than optimum. A bad choice for initial head also can cause the cell to oscillate between wet and dry.

Another user-specified option is the solver iteration interval for attempting to wet cells. For example, if the interval is four, then cell wetting is attempted every four iterations. This can prevent fluctuations in heads that can occur in iterative solvers in the next few iterations after cells are converted to wet from inappropriately triggering more cell wetting. All of these options are included to help make the solution process more stable. Refer to McDonald and others (1992) for more information about solution instability and how to deal with it.

\section{Applicability and Limitations of Simulation Options}

The options for calculation of horizontal conductance under water-table conditions and under perched conditions were developed on the assumption that each model layer corresponds to a distinct aquifer or permeable horizon, and that these horizons are separated by distinct units of low permeability. Use of these options where these conditions are not satisfied may lead to a variety of problems and inaccuracies in simulation. Thus, care should be exercised in the decision on whether or not to use these options. For example, if the option for horizontal conductance calculation under water-table conditions is used where a water-table aquifer is represented by several model layers, and the water table is expected to traverse more than one layer during simulation, incorrect conversion of cells to a no-flow condition may occur as iterations are carried out. If the wetting option is not used, conversion to no flow is irreversible. The wetting option allows conversion to no flow to be reversed, yet conversion back and forth can sometimes repeatedly occur so that solver convergence is not achieved. These types of issues with wetting and drying can normally be eliminated by using the NewtonRaphson formulation.

\section{Horizontal Flow Barrier Package}

The capability to simulate thin, vertical, and low-permeability geologic features (barriers) was previously added to MODFLOW with the introduction of the Horizontal Flow Barrier (HFB) Package (Hsieh and Freckleton, 1993). This package has been incorporated into MODFLOW 6. Figure 4-8 shows a conceptualization of a model layer containing a low-permeability barrier. The HFB Package adjusts the horizontal hydraulic conductance computed by the internal flow package to account for the presence of the barrier. The HFB Package is not a complete internal flow package, but rather, it acts as a supplement to the NPF Package.

In the HFB Package, a barrier is conceptualized as being located at the shared interface between two finitedifference cells that are connected horizontally (fig. 4-8). The barrier is further conceptualized as being in series with the horizontal conductance computed by the NPF Package. Using equation 4-23, the equivalent conductance for the additional conductance of the barrier, $C_{B a r r i e r}$, in series with the original horizontal conductance between cells $n$ and $m, C_{n, m}^{\text {original }}$ (fig. 4-9) is

$$
C_{n, m}=\frac{C_{n, m}^{\text {original }} C_{\text {Barrier }}}{C_{n, m}^{\text {original }}+C_{\text {Barrier }}}
$$


$\boldsymbol{A}$

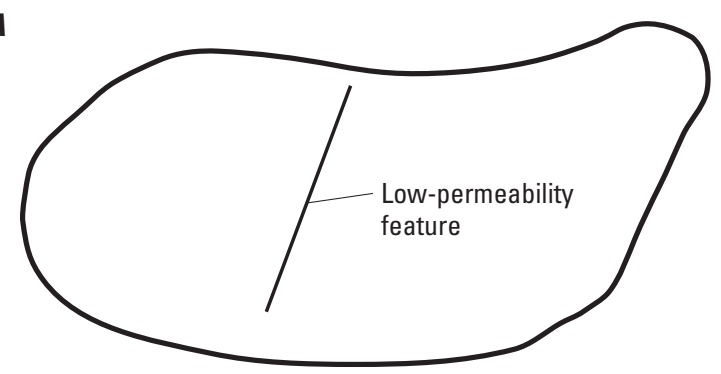

B

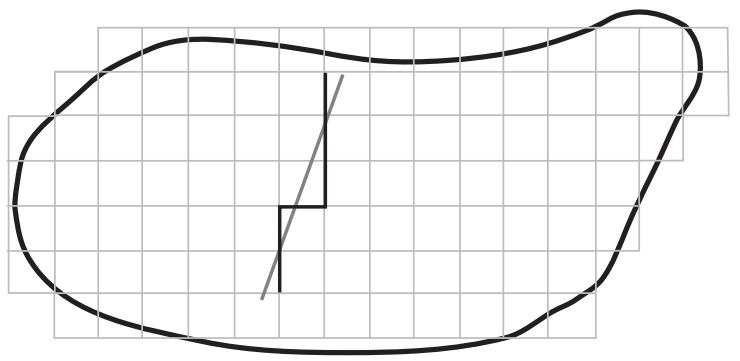

Figure 4-8. Diagram showing a low-permeability feature and its implementation in a model grid. $A$, shows the feature within the model domain, and $B$, shows the low-permeability feature represented in the grid as a series of six horizontal flow barriers. Figure modified from Hsieh and Freckleton (1993).

$C_{\text {Barrier }}$ is computed (from eq. 4-14) as

$$
C_{\text {Barrier }}=\frac{K_{\text {Barrier }} \Delta v_{\text {Barrier }} \Delta w_{n, m}}{L_{\text {Barrier }}}
$$

where $K_{\text {Barrier }}$ is hydraulic conductivity of the barrier, $\Delta v_{\text {Barrier }}$ is vertical thickness of the barrier, which is the average saturated thickness of the two cells, and $L_{\text {Barrier }}$ is the distance across the barrier in the flow direction. The HFB Package requires as input the "hydraulic characteristic" for a barrier, which is defined here as $\frac{K_{\text {Barrier }}}{L_{\text {Barrier }}}$. The HFB Package cannot be used to simulate barriers to vertical flow.

\section{Ghost Node Correction Package}

In some cases, a connection between two cells may violate the CVFD requirement regarding the geometric properties of the connection. As described by Panday and others (2013), MODFLOW-USG introduced a "ghost node correction" package that could be turned on or off based on user preferences. The underlying equations of the Ghost Node Correction (GNC) Package in MODFLOW-USG have also been implemented as the GNC Package in MODFLOW 6. This section summarizes the GNC Package in MODFLOW 6 and is based largely on the description by Panday and others (2013).

The CVFD equation is a second-order approximation when the line connecting two cells is perpendicular to the shared face and intersects the midpoint of the shared face (Dehotin and others, 2010). This condition is satisfied for a simple grid composed of combinations of equilateral triangles, rectangles, and other regular higher order polygons (regular pentagons, regular hexagons, and so forth). However, the CVFD equation is a lower order approximation when a line drawn between two connected nodes does not intersect the center of the shared face at a right angle. The consequence is an error in the simulated heads and flows (Edwards, 1996). 


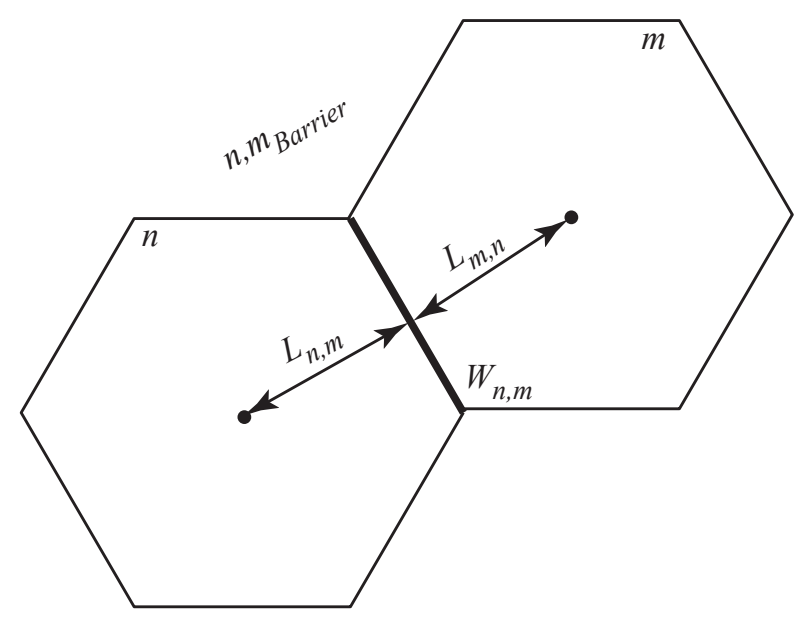

Figure 4-9. Diagram showing a horizontal flow barrier separating cell $n$ from its horizontal neighbor cell $m$.

This is the case, for example, for rectangular nested grids in which the face length of a parent grid is divided among the various nested child grids. Use of a ghost node, as shown in figure 4-10, can partially correct for this flux error and maintain local mass conservation. The term "ghost node" was introduced by Dickinson and others (2007) to indicate the fictitious node at a location at which the variable of interest (in this case, groundwater head) should be evaluated and used for computation of flow between parent and child grids.

The GNC Package is an optional addition that provides higher order correction terms to a MODFLOW 6 simulation; therefore, the GNC Package provides an adjustment to the already assembled coefficients of the CVFD matrix equations to include the GNC adjustment. Ghost nodes are not directly added to the system of equations, and consequently, head values are never explicitly calculated at the ghost node locations. Instead, the concept of a ghost node is implicitly built into the system of equations through interpolation factors with surrounding cells.

\section{Standard Formulation}

As described earlier in this chapter, MODFLOW 6 and its predecessors are based on a standard formulation in which the flow between two cells, $n$ and $m$, is

$$
Q_{n, m}=C_{n, m}\left(h_{m}-h_{n}\right)
$$

Using the nested rectangular grid of 4-10 as an example, flow between cells $n$ and $m$ can be improved by replacing $h_{n}$ with the head at a location that better conforms to the CVFD approximation. By doing so, the corrected equation for flow between cells $n$ and $m$ is

$$
Q_{n, m}=C_{n, m}\left(h_{m}-h_{\bar{n}}\right),
$$

where $h_{\bar{n}}$ is the head at the ghost node location. As shown in figure 4-10, a simple approach for expressing $h_{\bar{n}}$ is to use a distance weighted average between $n$ and $j$. The distance-weighted average assumes a linear head 


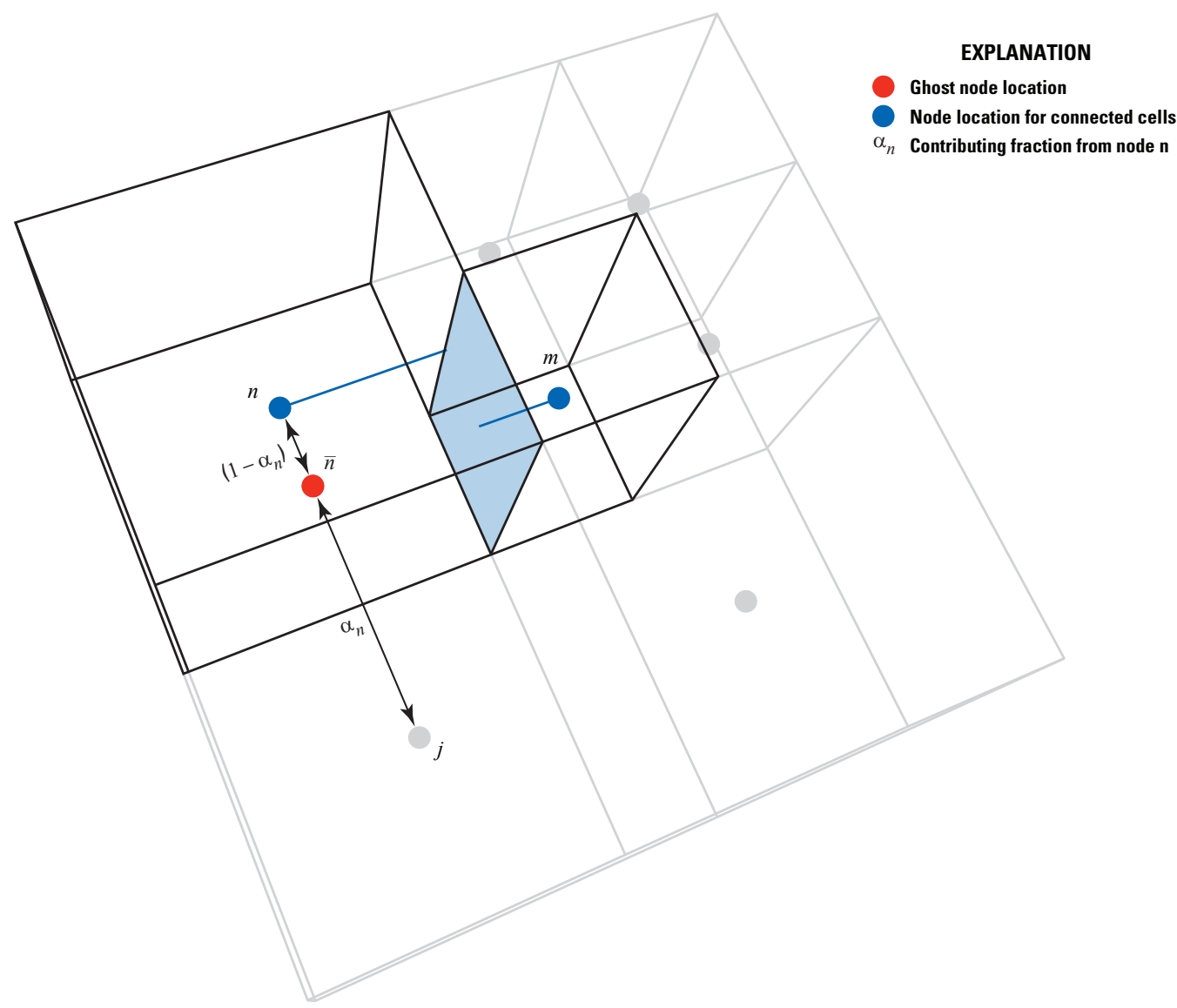

Figure 4-10. Diagram showing ghost node conceptualization for a nested grid. Figure modified from Panday and others (2013). 


\section{4-24 Documentation for the MODFLOW 6 Groundwater Flow Model}

profile between $n$ and $j$. In the general case, the head at the ghost node can be expressed as a function of any number of nearby $j$ contributing cells. Thus, a general equation for the ghost node head is

$$
h_{\bar{n}}=\alpha_{n} h_{n}+\sum_{j \in \eta_{n}} \alpha_{j} h_{j}
$$

where $\alpha_{n}$ is the contributing fraction of cell $n, \sum_{j \in \eta_{n}}$ is the summation over all $j$ contributing cells, and $\alpha_{j}$ is the contributing fraction for each contributing cell, $j$. The sum of all the contributing fractions $\alpha_{j}$ and $\alpha_{n}$ is one and

$$
\sum_{j \in \eta_{n}} \alpha_{j}=1-\alpha_{n}
$$

The contributing fractions are specified by the user and may be computed by linear interpolation, or by use of a Darcy-weighted interpolation as described by Mehl and Hill (2013). Other methods of evaluating the correction term have been presented by Dehotin and others (2010) and Edwards (1996).

Substitution of equation 4-61 into 4-60 gives the corrected flow equation as

$$
Q_{n, m}=C_{n, m}\left(h_{m}-\alpha_{n} h_{n}-\sum_{j \in \eta_{n}} \alpha_{j} h_{j}\right)
$$

Equation 4-63 is implemented in MODFLOW 6 as a correction to the conductance-based form (eq. 4-59). This correction term, $\Delta Q_{n, m}^{G N C}$, is formed by subtracting 4-59 from 4-60, which yields

$$
\Delta Q_{n, m}^{G N C}=C_{n, m}\left[\left(1-\alpha_{n}\right) h_{n}-\sum_{j \in \eta_{n}} \alpha_{j} h_{j}\right] .
$$

Substitution of equation 4-62 into equation 4-64 and simplification results in

$$
\Delta Q_{n, m}^{G N C}=C_{n, m} \sum_{j \in \eta_{n}} \alpha_{j}\left(h_{n}-h_{j}\right)
$$

Thus, the corrected flow is formulated in MODFLOW 6 as

$$
Q_{n, m}=C_{n, m}\left(h_{m}-h_{n}\right)+\Delta Q_{n, m}^{G N C}
$$

where the first term on the right-hand side is formulated by the NPF Package and the second term (eq. 4-65) is formulated by the GNC Package.

The effects of ghost nodes are added to the system of equations using either an implicit approach, in which the number of connections in the coefficient matrix is expanded to accommodate the additional connections, or as an explicit approach, in which the correction is applied to the right-hand side. To add ghost node corrections using the implicit approach, the product of contributing fraction $\alpha_{j}$ and $C_{n, m}$ is added to the diagonal of the coefficient matrix for node $n$ and subtracted from the off-diagonal term of the coefficient matrix corresponding to node $j$. This correction is done for every ghost node correction for cell $n$. 
Ghost node corrections can be formulated explicitly by leaving the coefficient matrix unchanged and subtracting and adding the product of the contributing fraction $\alpha_{j}, C_{n, m}$, and the head difference between nodes $n$ and $j\left(h_{n}-h_{j}\right)$ from and to the right-hand side of nodes $n$ and $m$, respectively. The explicit ghost node correction approach would be appropriate for a model using the standard formulation for the NPF and storage (STO) packages, all boundary packages, and a symmetric linear acceleration method (for example, the preconditioned conjugate gradient method).

\section{Newton-Raphson Formulation}

If the Newton-Raphson formulation is used with the NPF Package, then the coefficient matrix also needs to be updated with derivative terms based on equation $4-65$. The upstream weighted form of the ghost node correction term is

$$
\Delta Q_{n, m}^{G N C}=S_{F_{n, m}}^{*} C_{n, m}^{0} \sum_{j \in \eta_{n}} \alpha_{j}\left(h_{n}-h_{j}\right)
$$

The derivatives with respect to $h_{n}, h_{m}$, and $h_{j}$ are obtained from equation 4-67 as

$$
\begin{aligned}
& \frac{\partial \Delta Q_{n, m}^{G N C}}{\partial h_{n}}=S_{F_{n, m}}^{*} C_{n, m}^{0} \sum_{j \in \eta_{n}} \alpha_{j}+\sum_{j \in \eta_{n}} \frac{\partial S_{F_{n, m}}^{*}}{\partial h_{n}} \alpha_{j} C_{n, m}^{0}\left(h_{n}-h_{j}\right), \\
& \frac{\partial \Delta Q_{n, m}^{G N C}}{\partial h_{m}}=\sum_{j \in \eta_{n}} \frac{\partial S_{F_{n, m}}^{*}}{\partial h_{m}} \alpha_{j} C_{n, m}^{0}\left(h_{n}-h_{j}\right),
\end{aligned}
$$

and

$$
\frac{\partial \Delta Q_{n, m}^{G N C}}{\partial h_{j}}=-S_{F_{n, m}}^{*} C_{n, m}^{0} \sum_{j \in \eta_{n}} \alpha_{j} .
$$

$\frac{\Delta Q_{n, m}^{G N C}}{\partial h_{j}}$ in equation 4-70 does not include a $\frac{\partial S_{F_{n, m}}^{*}}{\partial h_{j}}$ term because $C_{n, m}$ is not dependent on the head in cell $j$. Note for upstream weighting that

$$
\frac{\partial S_{F n, m}^{*}}{\partial h_{n}}= \begin{cases}\frac{\partial S_{F n}^{*}}{\partial h_{n}} & \text { if } n \text { is upstream } \\ 0 & \text { if } m \text { is upstream }\end{cases}
$$

and

$$
\frac{\partial S_{F n, m}^{*}}{\partial h_{m}}=\left\{\begin{array}{ll}
\frac{\partial S_{F m}^{*}}{\partial h_{m}} & \text { if } m \text { is upstream } \\
0 & \text { if } n \text { is upstream }
\end{array} .\right.
$$




\section{4-26 Documentation for the MODFLOW 6 Groundwater Flow Model}

The ghost node correction can be implemented in a fully implicit manner by writing the correction terms in the form of equation $2-30$ as

$$
\begin{array}{r}
\frac{\partial \Delta Q_{n, m}^{G N C}}{\partial h_{n}} h_{n}^{k}+\frac{\partial \Delta Q_{n, m}^{G N C}}{\partial h_{m}} h_{m}^{k}+\sum_{j \in \eta_{n}} \frac{\partial \Delta Q_{n, m}^{G N C}}{\partial h_{j}} h_{j}^{k}= \\
-\Delta Q_{n, m}^{G N C}+\frac{\partial \Delta Q_{n, m}^{G N C}}{\partial h_{n}} h_{n}^{k-1}+\frac{\partial \Delta Q_{n, m}^{G N C}}{\partial h_{m}} h_{m}^{k-1}+\sum_{j \in \eta_{n}} \frac{\partial \Delta Q_{n, m}^{G N C}}{\partial h_{j}} h_{j}^{k-1} .
\end{array}
$$

Substitution of equations 4-68 through 4-70 into equation 4-73 results in the following general expansion of the Newton-Raphson formulation for the ghost node correction as

$$
\begin{array}{r}
{\left[S_{F_{n, m}}^{*} C_{n, m}^{0} \sum_{j \in \eta_{n}} \alpha_{j}+\frac{\partial S_{F n, m}^{*}}{\partial h_{n}} C_{n, m}^{0} \sum_{j \in \eta_{n}} \alpha_{j}\left(h_{n}^{k-1}-h_{j}^{k-1}\right)\right] h_{n}^{k}+} \\
{\left[\sum_{j \in \eta_{n}} \frac{\partial S_{F_{n, m}}^{*}}{\partial h_{m}} \alpha_{j} C_{n, m}^{0}\left(h_{n}^{k-1}-h_{j}^{k-1}\right)\right] h_{m}^{k}+\sum_{j \in \eta_{n}}\left(-S_{F n, m}^{*} C_{n, m}^{0} \alpha_{j}\right) h_{j}^{k}=} \\
\quad-S_{F_{n, m}}^{*} C_{n, m}^{0} \sum_{j \in \eta_{n}} \alpha_{j}\left(h_{n}^{k-1}-h_{j}^{k-1}\right)+ \\
{\left[S_{F_{n, m}}^{*} C_{n, m}^{0} \sum_{j \in \eta_{n}} \alpha_{j}+\frac{\partial S_{F n, m}^{*}}{\partial h_{n}} C_{n, m}^{0} \sum_{j \in \eta_{n}} \alpha_{j}\left(h_{n}^{k-1}-h_{j}^{k-1}\right)\right] h_{n}^{k-1}+} \\
{\left[\sum_{j \in \eta_{n}} \frac{\partial S_{F_{n, m}}^{*}}{\partial h_{m}} \alpha_{j} C_{n, m}^{0}\left(h_{n}^{k-1}-h_{j}^{k-1}\right)\right] h_{m}^{k-1}+} \\
\sum_{j \in \eta_{n}}\left(-S_{F n, m}^{*} C_{n, m}^{0} \alpha_{j}\right) h_{j}^{k-1} .
\end{array}
$$

Simplification of equation $4-74$ results in

$$
\begin{array}{r}
{\left[S_{F_{n, m}}^{*} C_{n, m}^{0} \sum_{j \in \eta_{n}} \alpha_{j}+\frac{\partial S_{F n, m}^{*}}{\partial h_{n}} C_{n, m}^{0} \sum_{j \in \eta_{n}} \alpha_{j}\left(h_{n}^{k-1}-h_{j}^{k-1}\right)\right] h_{n}^{k}+} \\
{\left[\sum_{j \in \eta_{n}} \frac{\partial S_{F_{n, m}}^{*}}{\partial h_{m}} \alpha_{j} C_{n, m}^{0}\left(h_{n}^{k-1}-h_{j}^{k-1}\right)\right] h_{m}^{k}+\sum_{j \in \eta_{n}}\left(-S_{F n, m}^{*} C_{n, m}^{0} \alpha_{j}\right) h_{j}^{k}=} \\
\\
{\left[\frac{\partial S_{F n, m}^{*}}{\partial h_{n}} C_{n, m}^{0} \sum_{j \in \eta_{n}} \alpha_{j}\left(h_{n}^{k-1}-h_{j}^{k-1}\right)\right] h_{n}^{k-1}+} \\
{\left[\sum_{j \in \eta_{n}} \frac{\partial S_{F_{n, m}}^{*}}{\partial h_{m}} \alpha_{j} C_{n, m}^{0}\left(h_{n}^{k-1}-h_{j}^{k-1}\right)\right] h_{m}^{k-1} .}
\end{array}
$$

Two of the terms in equation 4-75 are added as part of the ghost node standard formulation (the first term in the coefficient multiplied by $h_{n}^{k}$ and the coefficient multiplied by $h_{j}^{k}$ ). The remaining terms are added specifically for the Newton-Raphson formulation of the ghost node correction. Depending on which cell is upstream, 
either $n$ or $m$, several terms in equation 4-74 are zero as indicated by equations 4-71 and 4-72. For example, when $n$ is upstream, then equation $4-75$ reduces to

$$
\begin{array}{r}
{\left[S_{F_{n, m}}^{*} C_{n, m}^{0} \sum_{j \in \eta_{n}} \alpha_{j}+\frac{\partial S_{F n, m}^{*}}{\partial h_{n}} C_{n, m}^{0} \sum_{j \in \eta_{n}} \alpha_{j}\left(h_{n}^{k-1}-h_{j}^{k-1}\right)\right] h_{n}^{k}+} \\
\sum_{j \in \eta_{n}}\left(-S_{F n, m}^{*} C_{n, m}^{0} \alpha_{j}\right) h_{j}^{k}=\frac{\partial S_{F n, m}^{*}}{\partial h_{n}} C_{n, m}^{0} \sum_{j \in \eta_{n}} \alpha_{j}\left(h_{n}^{k-1}-h_{j}^{k-1}\right) h_{n}^{k-1} .
\end{array}
$$

Conversely, when $m$ is upstream, equation $4-75$ reduces to

$$
\begin{gathered}
\left(S_{F_{n, m}}^{*} C_{n, m}^{0} \sum_{j \in \eta_{n}} \alpha_{j}\right) h_{n}^{k}+\left[\sum_{j \in \eta_{n}} \frac{\partial S_{F_{n, m}}^{*}}{\partial h_{m}} \alpha_{j} C_{n, m}^{0}\left(h_{n}^{k-1}-h_{j}^{k-1}\right)\right] h_{m}^{k}+ \\
\sum_{j \in \eta_{n}}\left(-S_{F n, m}^{*} C_{n, m}^{0} \alpha_{j}\right) h_{j}^{k}=\left[\sum_{j \in \eta_{n}} \frac{\partial S_{F_{n, m}}^{*}}{\partial h_{m}} \alpha_{j} C_{n, m}^{0}\left(h_{n}^{k-1}-h_{j}^{k-1}\right)\right] h_{m}^{k-1} .
\end{gathered}
$$

The explicit ghost node correction can be thought of as a specified flow boundary condition that corrects the conductance-based flow equation between cells $n$ and $m$ (eq. 4-59). As a result, the derivative of equation 4-67 with respect to $h_{n}$ and $h_{m}$ is

$$
\frac{\partial \Delta Q_{n, m}^{G N C}}{\partial h_{n}}=\sum_{j \in \eta_{n}} \frac{\partial S_{F_{n, m}}^{*}}{\partial h_{n}} \alpha_{j} C_{n, m}^{0}\left(h_{n}-h_{j}\right),
$$

and

$$
\frac{\partial \Delta Q_{n, m}^{G N C}}{\partial h_{m}}=\sum_{j \in \eta_{n}} \frac{\partial S_{F_{n, m}}^{*}}{\partial h_{m}} \alpha_{j} C_{n, m}^{0}\left(h_{n}-h_{j}\right),
$$

where equations 4-71 and 4-72 still apply for upstream weighting.

The assembly of the Newton-Raphson terms for the explicit ghost node correction option is identical to the implicit ghost node correction. If $n$ is upstream, the right-hand side of equation 4-78 is subtracted from the diagonal of the coefficient matrix and is added to the off-diagonal term of the coefficient for row $n$, and the product of the derivative and the current head in node $n$ is added to the right-hand side of row $n$ and subtracted from the right-hand side of row $m$. If $m$ is upstream, the right-hand side of equation 4-79 is added to the diagonal of the coefficient matrix and subtracted from the off-diagonal term of the coefficient for row $m$ and the product of the derivative and the current head in node $m$ is added to the right-hand side of row $n$ and subtracted from the right-hand side of row $m$. 


\section{Chapter 5. Storage}

This chapter documents the storage (STO) package that simulates the contribution of confined and unconfined storage changes to the groundwater flow equation in MODFLOW 6. There can be only one STO Package specified for a GWF Model. The general equation for storage contributions to the groundwater flow equation is

$$
Q_{S T O}=Q_{S S}+Q_{S y}
$$

where $Q_{S S}$ is the volumetric flow rate from specific storage $\left(L^{3} / T\right)$ and $Q_{S y_{n}}$ is the volumetric flow rate from specific yield $\left(L^{3} / T\right)$.

A distinction is made in MODFLOW 6 between cells in which storage terms are limited to the contribution from specific storage and remain constant throughout the simulation, and those cells in which the storage terms may "convert" from a specific storage value to a combination of specific storage and specific yield values, or conversely, from a combination of specific storage and specific yield values to a specific storage value, as the water level in a cell falls below or rises above the top of the cell. Unlike previous versions of MODFLOW but consistent with MODFLOW-NWT and MODFLOW-USG, specific storage contributes to storage changes when the water levels exceed the bottom of a cell rather than the top of a cell. A user-specified code is specified for each GWF model cell to determine if storage contributions in a cell are limited to contributions from specific storage (confined conditions) throughout the simulation (nonconvertible) or convertible (a combination of confined and unconfined conditions using specific storage and specific yield).

In the storage package, individual stress periods are specified by the user to be steady state or transient. For steady-state stress periods, storage terms are not added to the groundwater flow equation (eq. 2-21). When all stress periods in a simulation are steady state, the storage package file does not need to be included in the input data.

\section{Specific Storage}

For a cell with a water-level above the bottom of the cell, the storage contribution to the groundwater flow equation is based upon a direct application of the storage term in equation 2-20. The expression for contribution of water from specific storage to a single cell is

$$
Q_{S S_{n}}=\frac{S S_{n} A_{n}\left(T O P_{n}-B O T_{n}\right)}{t-t_{\text {old }}}\left(S_{F_{n}}^{t_{\text {old }}} H O L D_{n}-S_{F_{n}}^{t} h_{n}^{t}\right)
$$

Cell saturation varies from zero when the water level is at or below the bottom of the cell to one when the water level is at or above the top of the cell. For cells that cannot "convert," $S_{F_{n}}$ is one for all water-table elevations, and storage calculations are identical to previous versions of MODFLOW (for example, MODFLOW2005).

The notation $S C 1_{n}$ is introduced where $S C 1_{n}=S S_{n}\left(T O P_{n}-B O T_{n}\right)$. The term $S C 1_{n}$ is the "storage capacity" or the "primary storage capacity" of cell $n$; the "primary" designation is used to distinguish $S C 1_{n}$ from a secondary storage capacity, which is used when a cell is specified by the user to be "convertible," as explained in the following section. By using the concept of storage capacity, equation 5-2 can be rewritten as

$$
Q_{S S_{n}}=\frac{S C 1_{n}}{t-t^{\text {old }}}\left(S_{F_{n}}^{t_{\text {old }}} H O L D_{n}-S_{F_{n}}^{t} h_{n}^{t}\right)
$$




\section{5-2 Documentation for the MODFLOW 6 Groundwater Flow Model}

The approach used to represent the contribution from specific storage to the groundwater flow equation for the standard and Newton-Raphson formulation is detailed below. To allow for a smooth transition from dry (water level at or below the bottom of a cell) to fully saturated (water level at or above the top of the cell) conditions, quadratic smoothing is applied to the saturated cell fraction $\left(S_{F}\right)$ over a small interval when the water level approaches the top or bottom of a cell. In cases where the Newton-Raphson formulation is not used for specific storage calculations, a linear relation between water level and the saturated cell fraction is used and $\Omega$ in equation $4-5$ is defined to be zero.

\section{Standard Formulation}

For the case where the water level in cell $n$ is above the bottom of the cell for the previous time step $\left(t_{\text {old }}\right)$ and the current iteration $(k)$, the contribution of specific storage to the groundwater flow equations is defined as

$$
Q_{S S_{n}}^{k}=\frac{S C 1_{n}}{t-t_{\text {old }}}\left(S_{F_{n}}^{t_{\text {old }}} H O L D_{n}-S_{F_{n}}^{k-1} h_{n}^{k}\right)
$$

Rearranging equation 5-4 to move terms dependent on the current value of $h$ to the left side of equation 2-24 and terms dependent on the head at the end of the last time step to the right-hand side of equation 2-24 results in

$$
-\frac{S C 1_{n} S_{F_{n}}^{k-1}}{t-t_{\text {old }}} h_{n}^{k}=-\frac{S C 1_{n} S_{F_{n}}^{t_{\text {old }}}}{t-t_{\text {old }}} H O L D_{n}
$$

\section{Newton-Raphson Formulation}

The derivative of equation 5-4 with respect to $h_{n}$ is

$$
\frac{\partial Q_{S S_{n}}}{\partial h_{n}}=-\frac{S C 1_{n} S_{F_{n}}}{t-t_{\text {old }}}-\frac{S C 1_{n}}{t-t_{\text {old }}} \frac{\partial S_{F_{n}}}{\partial h_{n}} h_{n}^{k-1} .
$$

The fully implicit form of the Newton-Raphson formulation for the contribution of specific storage in cell $n$ in the form of equation $2-30$ is

$$
\frac{\partial Q_{S S_{n}}}{\partial h_{n}} h_{n}^{k}=-Q_{S S_{n}}+\frac{\partial Q_{S S_{n}}}{\partial h_{n}} h_{n}^{k-1} .
$$

Substitution of equations 5-4 and 5-6 into equation 5-7 results in the following general expression of the Newton-Raphson formulation for the contribution of specific storage to cell $n$ as:

$$
\begin{array}{r}
\left(-\frac{S C 1_{n} S_{F_{n}}^{k-1}}{t-t_{\text {old }}}-\frac{S C 1_{n}}{t-t_{\text {old }}} \frac{\partial S_{F_{n}}}{\partial h_{n}} h_{n}^{k-1}\right) h_{n}^{k}= \\
-\frac{S C 1_{n}}{t-t_{\text {old }}}\left(S_{F_{n}}^{t_{\text {old }}} H O L D_{n}-S_{F_{n}}^{k-1} h_{n}^{k-1}\right)+\left(-\frac{S C 1_{n} S_{F_{n}}^{k-1}}{t-t_{\text {old }}}-\frac{S C 1_{n}}{t-t_{\text {old }}} \frac{\partial S_{F_{n}}}{\partial h_{n}} h_{n}^{k-1}\right) h_{n}^{k-1} .
\end{array}
$$


Simplification of equation $5-8$ results in

$$
\left(-\frac{S C 1_{n} S_{F_{n}}^{k-1}}{t-t_{\text {old }}}-\frac{S C 1_{n}}{t-t_{\text {old }}} \frac{\partial S_{F_{n}}}{\partial h_{n}} h_{n}^{k-1}\right) h_{n}^{k}=-\frac{S C 1_{n} S_{F_{n}}^{t_{\text {old }}}}{t-t_{\text {old }}} H O L D_{n}-\left(\frac{S C 1_{n}}{t-t_{\text {old }}} \frac{\partial S_{F_{n}}}{\partial h_{n}} h_{n}^{k-1}\right) h_{n}^{k-1} .
$$

The $\frac{S C 1_{n} S_{F_{n}}^{k-1}}{t-t_{\text {old }}}$ and $\frac{S C 1_{n} S_{F n}^{t_{\text {old }}}}{t-t_{\text {old }}} H O L D_{n}$ terms in equation 5-9 are subtracted from the diagonal of the coefficient matrix and the right-hand side of equation 2-24 during the standard formulation, respectively. The Newton-Raphson formulation is completed by augmenting the coefficient matrix with the second term on the right-hand side of equation 5-6 and adding the product of the second term on the right-hand side of equation 5-6 and the current head to the right-hand side of equation 2-24.

\section{Specific Yield}

The primary storage capacity described above, $S C 1_{n}$, is adequate for simulations in which specific storage in a cell is the only storage contribution to a cell throughout the course of a simulation; however, if the water level is below the top of a cell during a simulation, then the cell is under a water-table condition, and a "secondary storage capacity" $\left(S C 2_{n}\right)$ calculated using specific yield $\left(S y_{n}\right)$ contributes to the groundwater flow equation. The "secondary storage capacity" contribution to groundwater flow is calculated as

$$
Q_{S y_{n}}=\frac{S C 2_{n}\left(T O P_{n}-B O T_{n}\right)}{t-t_{\text {old }}}\left(S_{F_{n}}^{t_{\text {old }}}-S_{F_{n}}^{t}\right)
$$

where $S C 2_{n}$ is $S y_{n} \cdot A_{n}$ for cell $n\left(\mathrm{~L}^{2}\right)$.

To allow use of the Storage Package with and without Newton-Raphson, specific yield storage calculations are formulated in terms of saturation. Similar to the specific storage approach, quadratic smoothing is applied in the specific yield storage approach to allow for a smooth transition from unconfined to confined conditions when the water level approaches the top or bottom of a cell. In cases where the Newton-Raphson formulation is not used for specific yield storage calculations, a linear relation between water level and the saturated cell fraction is used and $\Omega$ in equation $4-5$ is defined to be zero. The specific yield storage approach is detailed below.

\section{Standard Formulation}

For a cell under water-table conditions in the previous time step $\left(t_{\text {old }}\right)$ and the current iteration $(k)$, the contribution of specific yield to the groundwater flow equation is defined as

$$
Q_{S y_{n}}^{k}=\frac{S C 2_{n}\left(T O P_{n}-B O T_{n}\right)}{t-t_{\text {old }}}\left(S_{F_{n}}^{t_{\text {old }}}-S_{F_{n}}^{k}\right)
$$

As the linear equations in equation 2-20 are solved in terms of head and not saturation, equation 4-6 is used to formulate equation 5-11 in terms of $h$, which results in

$$
Q_{S y_{n}}^{k}=\frac{S C 2_{n}\left(T O P_{n}-B O T_{n}\right)}{t-t_{\text {old }}} S_{F_{n}}^{t_{\text {old }}}-\frac{S C 2_{n}\left(T O P_{n}-B O T_{n}\right)}{t-t_{\text {old }}} \frac{h_{n}^{k}-B O T_{n}}{\left(T O P_{n}-B O T_{n}\right)}
$$




\section{5-4 Documentation for the MODFLOW 6 Groundwater Flow Model}

Equation 5-12 is simplified and rearranged to get terms dependent on the current value of $h$ on the left-hand side of equation 2-24 and all other terms on the right-hand side of equation 2-24 to yield

$$
-\frac{S C 2_{n}}{t-t_{\text {old }}} h_{n}^{k}=-\frac{S C 2_{n}}{t-t_{\text {old }}} B O T_{n}-\frac{S C 2_{n}\left(T O P_{n}-B O T_{n}\right)}{t-t_{\text {old }}} S_{F_{n}}^{t_{\text {old }}}
$$

For cells where the head at the beginning of the current time step is at or above the top of the cell, the cell saturation fraction $\left(S_{F_{n}}\right)$ is one. In these cases, the specific yield storage contribution is known and equation 5-11 is subtracted from the right-hand side of equation 2-24 and specific yield storage terms are not added to the left-hand side of equation 2-24.

\section{Newton-Raphson Formulation}

The derivative of equation $5-11$ is

$$
\frac{\partial Q_{S y_{n}}}{\partial h_{n}}=-\frac{S C 2_{n}\left(T O P_{n}-B O T_{n}\right)}{t-t_{\text {old }}} \frac{\partial S_{F_{n}}}{\partial h_{n}} .
$$

The fully implicit form of the Newton-Raphson formulation for the contribution of specific yield storage in cell $n$ is

$$
\frac{\partial Q_{S y_{n}}}{\partial h_{n}} h_{n}^{k}=-Q_{S y_{n}}+\frac{\partial Q_{S y_{n}}}{\partial h_{n}} h_{n}^{k-1}
$$

Substitution of equations 5-11 and 5-14 into equation 5-15 results in the following general expression of the Newton-Raphson formulation for the contribution of specific yield storage to cell $n$ as

$$
\begin{array}{r}
{\left[-\frac{S C 2_{n}\left(T O P_{n}-B O T_{n}\right)}{t-t_{\text {old }}} \frac{\partial S_{F_{n}}}{\partial h_{n}}\right] h_{n}^{k}=} \\
-\left[\frac{S C 2_{n}\left(T O P_{n}-B O T_{n}\right)}{t-t_{\text {old }}} S_{F_{n}}^{t_{\text {old }}}+\frac{S C 2_{n}\left(T O P_{n}-B O T_{n}\right)}{t-t_{\text {old }}} S_{F_{n}}^{k-1}\right] \\
-\left[\frac{S C 2_{n}\left(T O P_{n}-B O T_{n}\right)}{t-t_{\text {old }}} \frac{\partial S_{F_{n}}}{\partial h_{n}}\right] h_{n}^{k-1} .
\end{array}
$$

The Newton-Raphson formulation for specific yield storage is constructed by augmenting the specific yield storage terms subtracted from the coefficient matrix as part of the standard formulation. The terms

$$
-\frac{S C 2_{n}\left(T O P_{n}-B O T_{n}\right)}{t-t_{\text {old }}} \frac{\partial S_{F_{n}}}{\partial h_{n}}+\frac{S C 2_{n}}{t-t_{\text {old }}}
$$

are added to the coefficient matrix for cell $n$ to complete the left-hand side of equation 2-24 for the NewtonRaphson formulation. The second term in equation 5-17 is the term that was subtracted from the diagonal of the coefficient matrix for cell $n$ during the standard formulation step. The terms

$$
\frac{S C 2_{n}\left(T O P_{n}-B O T_{n}\right)}{t-t_{\text {old }}} S_{F_{n}}^{k-1}+\frac{S C 2_{n}}{t-t_{\text {old }}} B O T_{n}
$$


are added to the right-hand side of equation 2-24. The first term in equation 5-18 completes the residual (eq. 5-11). The second term in equation 5-18 is the first term on the right-hand side of equation 5-13 that was subtracted from the right-hand side of equation 2-24 for cell $n$ during the standard formulation step.

For cells where the head at the beginning of the current time step is at or above the top of the cell, the derivative is zero and the left- and right-hand sides of equation 2-24 are not adjusted during the NewtonRaphson formulation. 


\section{Chapter 6. Conceptualization and Implementation of Stress Packages}

This chapter documents the conceptualization and implementation of the packages in the Groundwater Flow (GWF) Model that simulate hydrologic stresses to a groundwater system. Mathematically, these packages are boundary conditions. With the exception of the Time Varying Specified Head (CHD) Package, the packages behave similarly, according to a standard or Newton Raphson formulation. This chapter first describes the general way in which packages work for the conductance and Newton Raphson formulation; then the individual packages are described. The CHD Package is described first. Next, the remaining six stress packages are described: Well (WEL) Package, Recharge (RCH) Package, General-Head Boundary (GHB) Package, River (RIV) Package, Drain (DRN) Package, and Evapotranspiration (EVT) Package.

The behavior of the packages described in this chapter is similar to the behavior described by Harbaugh (2005) for MODFLOW-2005, but there are some important differences. One important difference is that multiple packages of the same type can be specified for a single GWF Model. Another difference is that a constanthead cell does not necessarily remain a constant-head cell for the entire simulation. A constant-head cell can convert to a variable-head cell during the simulation. Input files for all the stress packages can use list-based input in MODFLOW 6, even the RCH and EVT Packages, which have traditionally used array-based input. For the RCH and EVT Packages, this flexibility can be used to assign multiple entries per cell, with different recharge and evapotranspiration rates, to better represent land use or vegetation variations. Details on these types of functional differences are provided in the separate MODFLOW 6 user guide.

\section{Standard Formulation}

In chapter 2, the general CVFD flow equation for MODFLOW 6 was developed (eq. 2-21). This equation can be rewritten to highlight the contribution of boundary conditions to the CVFD flow equation as

$$
\begin{gathered}
\sum_{m \in \eta_{n}} C_{n, m} h_{m}+\left(-\sum_{m \in \eta_{n}} C_{n, m}-\sum_{n b \in n} C_{n b}-\frac{S S_{n} A_{n} \Delta v_{n}}{t-t_{\text {old }}}\right) h_{n}= \\
\sum_{n b \in n}\left(-Q_{n b}-C_{n b} H B_{n b}\right)-\frac{S S_{n} A_{n} \Delta v_{n}}{t-t_{\text {old }}} h^{t_{\text {old }}}
\end{gathered}
$$

where $C_{n b}$ is the conductance of boundary condition $n b$, which is connected to cell $n ; Q_{n b}$ is the specified flow rate of boundary condition $n b$, which is connected to cell $n$; and $H B_{n b}$ is the specified head of boundary condition $n b$, which is connected to cell $n$.

\section{Head-Dependent Boundaries}

As part of the standard formulation, $C_{n b}$ for each head-dependent boundary condition is subtracted from the diagonal of cell $n$ of equation 2-24. The product of $C_{n b}$ and $H B_{n b}$ is also subtracted from the right-hand side of equation 2-24 for the cell associated with every head-dependent boundary condition during the standard formulation.

\section{Specified-Flow Boundaries}

The specified flow rate, $Q_{n b}$, is subtracted from the right-hand side of equation 2-24 for the cell associated with every specified-flow boundary condition. The left-hand side of equation 2-24 is not modified for specified-flow boundary conditions during the standard formulation. 


\section{6-2 Documentation for the MODFLOW 6 Groundwater Flow Model}

\section{Newton-Raphson Formulation}

For boundary packages that use the Newton-Raphson formulation, the boundary conditions are assembled using Newton's method formulated in terms of $h$ instead of $\Delta h$.

\section{Head-Dependent Boundaries}

The general form of the linear equation for a head-dependent boundary condition is

$$
Q_{n b}=f_{n b} C_{n b}\left(H B_{n b}-h_{n}\right)
$$

where $f_{n b}$ is a smoothing function that varies from 0 to 1 over a defined head interval and results in zero flow when cell $n$ is dry. The fully-implicit form of the Newton-Raphson formulation for an individual headdependent boundary condition in cell $n$, based on equation $6-2$, is

$$
\frac{\partial Q_{n b}}{\partial h_{n}} h_{n}^{k}=-Q_{n b}^{k-1}+\frac{\partial Q_{n b}}{\partial h_{n}} h_{n}^{k-1}
$$

For the simple case where smoothing is not used ( $f_{n b}$ is always 1$)$, the derivative of equation 6-2, by applying the chain rule, is

$$
\frac{\partial Q_{n b}}{\partial h_{n}}=\frac{\partial}{\partial h_{n}} C_{n b}\left(H B_{n b}-h_{n}^{k-1}\right)=-C_{n b} .
$$

Substitution of equations 6-2 and 6-4 into equation 6-3 results in

$$
-C_{n b} h_{n}^{k}=-C_{n b}\left(H B_{n b}-h_{n}^{k-1}\right)-C_{n b} h_{n}^{k-1},
$$

which simplifies to

$$
-C_{n b} h_{n}^{k}=-C_{n b} H B_{n b},
$$

and is identical to the terms added to the left- and right-hand sides of equation 2-24 as part of the standard formulation.

For cases where $C_{n b}$ is a constant value and smoothing is applied, the derivative of equation 6-2 is

$$
\frac{\partial Q_{n b}}{\partial h_{n}}=\frac{\partial}{\partial h_{n}} f_{n b} C_{n b}\left(H B_{n b}-h_{n}\right)=-f_{n b} C_{n b}-\frac{\partial f_{n b}}{\partial h_{n}} C_{n b}\left(H B_{n b}-h_{n}\right) .
$$

Substitution of equations 6-2 and 6-7 into equation 6-3 results in

$$
\begin{array}{r}
{\left[-f_{n b} C_{n b}-\frac{\partial f_{n b}}{\partial h_{n}} C_{n b}\left(H B_{n b}-h_{n}^{k-1}\right)\right] h_{n}^{k}=} \\
-f_{n b} C_{n b}\left(H B_{n b}-h_{n}^{k-1}\right)+\left[-f_{n b}^{k-1} C_{n b}-\frac{\partial f_{n b}}{\partial h_{n}} C_{n b}\left(H B_{n b}-h_{n}^{k-1}\right)\right] h_{n}^{k-1} .
\end{array}
$$


Simplification of equation $6-8$ results in

$$
\begin{array}{r}
{\left[-f_{n b} C_{n b}-\frac{\partial f_{n b}}{\partial h_{n}} C_{n b}\left(H B_{n b}-h_{n}^{k-1}\right)\right] h_{n}^{k}=} \\
-f_{n b}^{k-1} C_{n b} H B_{n b}+\left[-\frac{\partial f_{n b}}{\partial h_{n}} C_{n b}\left(H B_{n b}-h_{n}^{k-1}\right)\right] h_{n}^{k-1} .
\end{array}
$$

The $f_{n b} C_{n b}$ and $f_{n b}^{k-1} C_{n b} H B_{n b}$ terms in equation 6-9 were subtracted from the diagonal of the coefficient matrix and right-hand side of equation 2-24 for cell $n$ during the standard formulation, respectively. The Newton-Raphson formulation is completed by augmenting the coefficient matrix with the second term on the right-hand side of equation 6-7 and adding the product of the second term on the right-hand side of equation 6-7 and the current head in cell $n$ to the right-hand side of equation 2-24.

For cases where $C_{n b}$ is a function of head but the derivative cannot be calculated analytically, a finitedifference approximation is used to calculate the derivative term. The finite-difference approximation of the derivative is

$$
\frac{\partial Q_{n b}}{\partial h_{n}}=\frac{C_{n b}^{+}\left[H B_{n b}-\left(h_{n}^{k-1}+\epsilon\right)\right]-C_{n b}\left(H B_{n b}-h_{n}^{k-1}\right)}{\epsilon},
$$

where $C_{n b}^{+}$is the boundary condition conductance at $h+\epsilon$ and $\epsilon$ is a small perturbation value. The conductance term was added to the diagonal of equation 2-24 for row $n$ as part of the standard formulation. As a result, the conductance is subtracted from the diagonal during the Newton-Raphson formulation. The NewtonRaphson formulation is completed by adding the derivative to the diagonal of row $n$ and adding the product of the derivative and the current head in cell $n$ along with the missing portion of the residual $\left(C_{n b} h_{n}^{k-1}\right)$ to the right-hand side of row $n$.

\section{Specified Flow Boundaries}

For specified flow boundaries where the flow is not a function of head, the derivative is 0 and neither the coefficient matrix nor the right-hand side needs to be modified. In cases where a specified flow boundary is a function of head, the specified flow is calculated as

$$
Q_{n b}=f_{n b} Q_{n b}^{0},
$$

where $Q_{n b}^{0}$ is the user-specified flow rate. The derivative of equation 6-11 is

$$
\frac{\partial Q_{n b}}{\partial h_{n}}=\frac{\partial f_{n b}}{\partial h_{n}} Q_{n b}^{0}
$$

For cases where the specified flow is a function of head but the derivative cannot be calculated analytically, the derivative of equation 6-11 is calculated using the finite-difference approximation, as shown in the following equation:

$$
\frac{\partial Q_{n b}}{\partial h_{n}}=\frac{Q_{n b}^{+}-Q_{n b}}{\epsilon}
$$




\section{6-4 Documentation for the MODFLOW 6 Groundwater Flow Model}

where $Q_{n b}^{+}$is the specified flow at $h_{n}+\epsilon$.

The fully implicit form of the Newton-Raphson formulation for specified-flow boundary condition $n b$ in the form of equation $2-30$ is

$$
\frac{\partial Q_{n b}}{\partial h_{n}} h_{n}^{k}=-Q_{n b}^{k-1}+\frac{\partial Q_{n b}}{\partial h_{n}} h_{n}^{k-1}
$$

$Q_{n b}$ was subtracted from the right-hand side of equation 2-24 of the row containing specified-flow boundary condition $n b$ during the standard formulation. The Newton-Raphson formulation is completed by adding the right-hand side of equation 6-12 or 6-13 to the diagonal of the coefficient matrix and adding the product of the right-hand side of equation 6-12 or 6-13 and the current head in cell $n$ to the right-hand side. 


\section{Specified Head Package}

Constant-head cells are designated in MODFLOW 6 using the CHD Package. This capability was originally developed by Leake and Prudic (1991). Harbaugh (2005) refers to the CHD Package as an option within the Basic Package, but the Basic Package no longer exists in MODFLOW 6. Thus, in MODFLOW 6, the CHD Package is referred to as a Hydrologic/Stress package, because it is most often used in this context. However, the CHD Package does not behave like other Hydrologic/Stress Packages; it does not add its effects to the system of equations like the other packages do. Instead, the CHD Package simply sets an internal integer code and head value according to user input. This internal integer code is stored with the GWF Model and indicates whether (1) the head varies with time (variable-head cell), (2) the head is constant (constant-head cell), or (3) no flow takes place within the cell (inactive cell). This internal code is used by the Numerical Solution to determine how the equation should be handled.

When the CHD Package is used, constant-head cells are read from the CHD input file as a list of cells. For each cell in the list, the CHD Package sets the internal code equal to negative one. For cells designated as constant head using the CHD Package, the head is also specified in the CHD file. If a cell that is designated as constant head in the CHD Package was already designated as constant head using another CHD Package assigned to the GWF Model, then the program terminates with an error. The CHD Package reads data every stress period, which makes changing the head at constant-head cells throughout a simulation possible. In previous MODFLOW versions, once a cell was identified as a constant-head cell, then it could not convert to an active cell in subsequent stress periods. This restriction has been eliminated in MODFLOW 6. 


\section{6-6 Documentation for the MODFLOW 6 Groundwater Flow Model}

\section{Well Package}

The Well (WEL) Package is designed to simulate features such as wells that withdraw water from or add water to the aquifer at a specified rate during a stress period, where the rate is independent of both the cell area and the head in the cell. The discussion in this section is developed on the assumption that the features to be simulated are actually wells, either discharging or recharging; however, the package can be used to simulate any features for which the volumetric recharge or discharge rate can be directly specified.

The flow rate, $Q W E L$, for a well is specified by the user as a fluid volume per unit time at which water is added to the aquifer. Negative values of $Q W E L$ are used to indicate well discharge (pumping), whereas positive values of $Q W E L$ indicate an injection well. Several data values are required for each well-the cellid in which the well is located and the flow rate $(Q W E L)$ of the well. The wells are redefined each stress period.

The WEL Package does not directly accommodate wells that are open to more than one node of the model. A well of this type, however, can be represented as a group of single-cell wells, each open to one of the nodes intersected by the multinode well, and each having an individual $Q W E L$ term specified for each stress period. If this approach is used, the flow rate of the multinode well must be divided or apportioned in some way among the individual nodes, externally to the model program. A common method for apportioning the well flow is to divide the flow rate according to the cell transmissivities, as follows:

$$
\frac{Q W E L_{n}}{Q W E L_{n b}}=\frac{T_{n}}{\sum T}
$$

where $Q W E L_{n}$ is the flow rate from cell $n$ to well $n b\left(\mathrm{~L}^{3} \mathrm{~T}^{-1}\right), Q W E L_{n b}$ is the total flow rate for well $n b$ $\left(\mathrm{L}^{3} \mathrm{~T}^{-1}\right), T_{n}$ is the transmissivity of cell $n\left(\mathrm{~L}^{2} \mathrm{~T}^{-1}\right)$, and $\sum T$ represents the sum of the transmissivities of all nodes penetrated by the well $\left(\mathrm{L}^{2} \mathrm{~T}^{-1}\right)$.

With the WEL Package, equation 6-15, or some other method of apportioning the well flow rate, must be implemented by the user externally to the program for each multilayer well, and for each stress period. This approach, in which a multilayer well is represented as a group of single layer wells, fails to take into account the interconnection between various layers provided by the well itself and the water level in the well, which drives flow from multiple layers, and is, thus, an incomplete representation of the problem. The Multi-Aquifer Well Package was created to directly simulate multilayer wells and is documented in the next chapter.

\section{Automatic Flow Reduction}

The WEL Package includes an option to reduce the withdrawal rate of a well as the water-table elevation in the cell approaches the cell bottom. The flow reduction occurs when the saturated cell thickness is less than a user-defined percentage $(\Phi)$ of the cell thickness. This "automatic flow reduction" capability is based on similar options implemented in MODFLOW-NWT and MODFLOW-USG (Niswonger and others, 2011; Panday and others, 2013). The option is based on equation 6-11 and is only applied to cells marked as convertible and are unconfined. If the flow reduction option is on and the flow rate for the well is negative (extraction), then the well pumping rate $(Q W E L)$ is calculated using

$$
Q W E L_{n b}=S_{W E L_{n b}} Q W E L_{n b}^{0}
$$




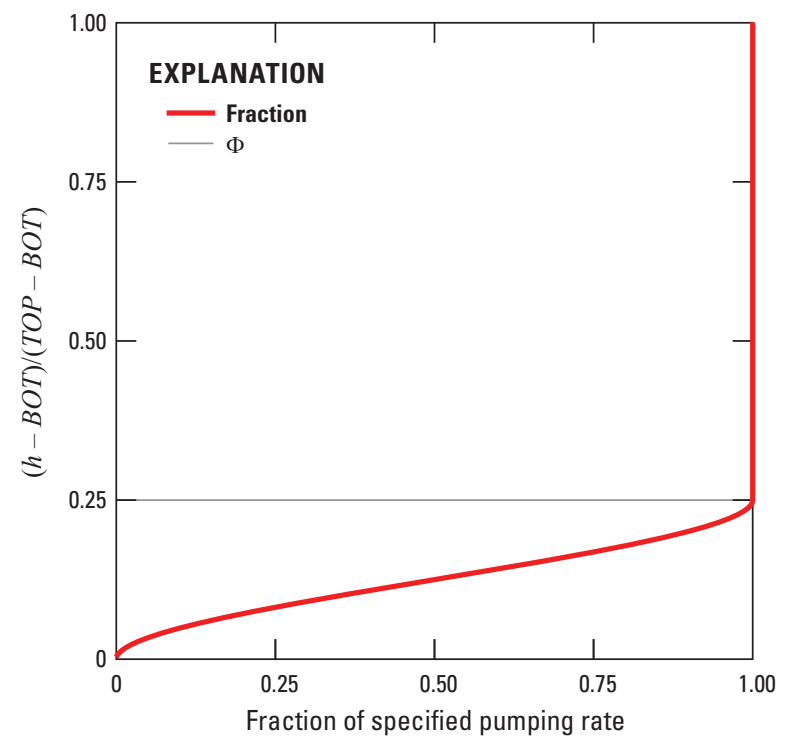

Figure 6-1. Graph showing the cubic function used to smoothly reduce specified groundwater withdrawal rates to zero when a cell dewaters, $\Phi=0.25$. Figure modified from Niswonger and others (2011).

where $S_{W E L_{n b}}$ is the fraction of the specified pumping rate for the cell containing well $n b$ and $Q W E L_{i}^{0}$ is the user-specified well pumping rate for well $n b$. If $Q W E L_{n b}^{0} \geq 0, S_{W E L_{n b}}$ is 1 . Otherwise, if $Q W E L_{n b}^{0}<0$ then $S_{W E L_{n b}}$ is defined as

$$
\begin{array}{rrr}
S_{W E L_{n b}}=\Delta v_{n}^{2}\left(\frac{-2}{\zeta_{n}^{3}} \Delta v_{n}+\frac{3}{\zeta_{n}^{2}}\right), & 0<\Delta v_{n}<\zeta_{n} \\
S_{W E L_{n b}}=1, & \Delta v_{n}>\zeta_{n} \\
S_{W E L_{n b}}=0, & \Delta v_{n} \leq 0,
\end{array}
$$

where $\zeta_{n}=\Phi\left(T O P_{n}-B O T_{n}\right), \Phi$ ranges between 0.0 and 1.0 , and typically is a small value, such as 0.25 or smaller. Figure 6-1 shows an example of the smoothing function used to reduce negative pumping rates to zero.

\section{Standard Formulation}

At each iteration, as the matrix equations are formulated, the value of $Q W E L_{n b}$ for each well is subtracted from the right-hand side of equation 2-24 for the cell containing that well. Where more than one well falls within a single cell, the calculation is repeated for each well as the right-hand side for that cell is assembled. Thus, the user specifies the flow rate associated with each individual well, and these are, in effect, summed within the program to obtain the total well rate for the cell. 


\section{6-8 Documentation for the MODFLOW 6 Groundwater Flow Model}

\section{Newton-Raphson Formulation}

The Newton-Raphson formulation of the Well Package is identical to the standard formulation, except when the optional automatic flow reduction capability is used and $Q W E L_{n b}$ is less than zero. When the optional automatic flow reduction capability is used and $Q W E L_{n b}$ is less than zero, the derivative is

$$
\begin{aligned}
\frac{\partial Q W E L_{n b}}{\partial h_{n}}=Q W E L_{n b}^{0}\left(-\frac{6}{\zeta_{n}^{3}} \Delta v_{n}^{2}+\frac{6}{\zeta_{n}^{2}} \Delta v_{n}\right), & 0<\Delta v_{n}<\zeta_{n} \\
\frac{\partial Q W E L_{n b}}{\partial h_{n}}=0, & \Delta v_{n}>\zeta_{n} \\
\frac{\partial Q W E L_{n b}}{\partial h_{n}}=0, & \Delta v_{n} \leq 0 .
\end{aligned}
$$

The fully implicit form of the Newton-Raphson formulation for discharge from well $n b$ in the form of equation $2-30$ is

$$
\frac{\partial Q W E L_{n b}}{\partial h_{n}} h_{n}^{k}=-Q W E L_{n b}^{k-1}+\frac{\partial Q W E L_{n b}}{\partial h_{n}} h_{n}^{k-1}
$$

The variable $Q W E L_{n b}$ was subtracted from the right-hand side of equation 2-24, the row for well $n b$, during the standard formulation. The Newton-Raphson formulation is completed by adding the right-hand side of equation 6-18 to the diagonal of the coefficient matrix, and adding the product of the right-hand side of equation 6-18 and the current head in cell $n$ to the right-hand side. 


\section{Recharge Package}

The Recharge $(\mathrm{RCH})$ Package is designed to simulate areally distributed recharge to the groundwater system. Most commonly, areal recharge occurs as a result of precipitation that percolates to the groundwater system. Recharge applied to the model is defined as

$$
Q R_{n b}=I_{n b} M_{n b} A_{n}
$$

where $Q R_{n b}$ is the recharge flow rate applied to node $n$ expressed as a fluid volume per unit time $\left(\mathrm{L}^{3} \mathrm{~T}^{-1}\right) ; I_{n b}$ is the recharge flux (in units of length per time, $\mathrm{LT}^{-1}$ ) applicable to the map area, $A_{n}$, of the cell; and $M_{n b}$ is an area multiplier (dimensionless) that can be used to scale $I_{n b}$ (for example, for altitude adjustment of rainfall data or to account for impervious areas in a cell).

Values of recharge flux, $I_{n b}$, are specified by the user at each stress period. These values of recharge flux are multiplied by horizontal cell areas, $A_{n}$, to obtain flow values of $Q R_{n b}$. The recharge flow rate, $Q R_{n b}$, is applied to the cell containing recharge boundary condition $n b$. In the simplest case, the cell containing the recharge boundary condition $n b$ will correspond to the top of the groundwater system and represent the uppermost cell in a vertical column; however, the vertical position of the water table, which is calculated during the simulation, may vary with horizontal location and with time as the water table rises and falls. For this reason, an option is required for applying recharge to the highest active cell when the standard formulation is used.

MODFLOW has typically had three options for specifying recharge: (1) apply recharge to layer 1, (2) apply recharge to a user-defined layer, and (3) apply recharge to the highest active cell. These options have been simplified into two options in MODFLOW 6. The default in MODFLOW 6 is equivalent to option $3-$ apply recharge to the highest active cell. This is the most commonly used approach and should be used for most applications. The first of these three options is a subset of option 2, and, therefore, these two options have been combined in MODFLOW 6. This option is called the FIXED_CELL option in MODFLOW 6. When the FIXED_CELL option is active, recharge will be applied to the cell specified by the user. If that cell is dry, then recharge to that cell will be lost, because it is not routed down to an active cell beneath it - the recharge is fixed to the specified cell.

For the default and FIXED_CELL options, recharge is never applied to constant-head cells. For the default option in the determination of the highest active layer, if a constant-head cell is encountered, then the recharge is not applied.

Careful consideration should be given to the problem under study and to the other options employed in the simulation before deciding if the FIXED_CELL option should be used in a given situation. For example, figure 6-2 shows a situation in which a cross-section model has been used to simulate a hypothetical problem involving recharge, seepage from a river, and seepage into a river (fig. 6-2). The river is simulated by constanthead cells. Using the provision described in chapter 4 for the standard formulation under water-table conditions, cells for which the computed head was lower than the bottom elevation were converted to no flow so that the uppermost variable-head cell in each vertical column contains the water table. This process yields the final distribution of variable-head, constant-head, and no-flow cells shown in figure 6-2B.

Figure 6-2C illustrates the recharge distribution for the default option with recharge applied to layer 1. This turns out to be the approach for this particular situation. Under this option, recharge enters the uppermost variable-head cell in each vertical column, except where constant-head cells have been used to represent the river. Thus, a continuous distribution of recharge to the water table is simulated.

Figure 6-2D illustrates the recharge distribution to the model if the FIXED_CELL option is used and recharge is applied to layer 1 . With this approach, recharge is permitted only to the top layer of the model. Thus, once the water-table shape has been simulated by the use of no-flow cells in the top layer, recharge to the vertical columns beneath those cells is shut off. This clearly fails to simulate the given system. 


\section{6-10 Documentation for the MODFLOW 6 Groundwater Flow Model}

A. Vertical cross section showing field situation with finite-difference grid superimposed

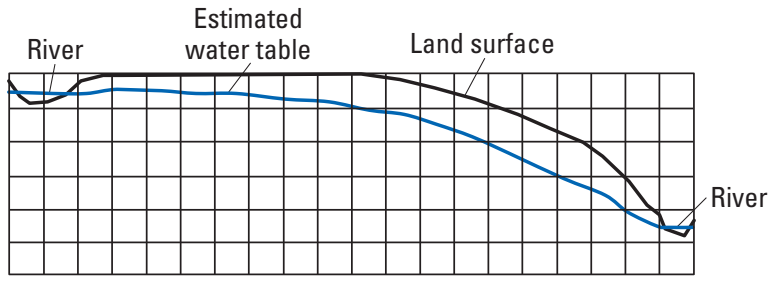

B. Status of cells at end of simulation

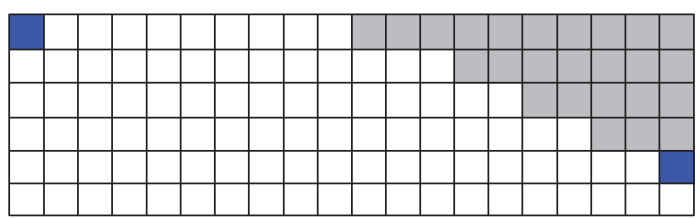

C. Cells that receive recharge under default option and recharge applied to all cells in layer 1

\begin{tabular}{|c|c|c|c|c|c|c|c|c|c|c|c|c|c|c|c|c|}
\hline $\mathbf{X}$ & $\overline{\mathbf{X}}$ & $\mathbf{X}$ & 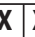 & \begin{tabular}{c|c}
$\mathrm{X}$ \\
\end{tabular} & $\mathbf{X}$ & $\mathbf{X}$ & $\mathbf{X}$ & & & & & & & & & \\
\hline & & & & & & & & & X & $\mathbf{X}$ & & & & & & \\
\hline & & & & & & & & & & & & & & & & \\
\hline & & & & & & & & & & & & & & $\mathbf{X}$ & & \\
\hline & & & & & & & & & & & & & & & & X \\
\hline & & & & & & & & & & & & & & & & \\
\hline
\end{tabular}

D. Cells that receive recharge FIXED_CELL option and recharge applied to all cells in layer 1

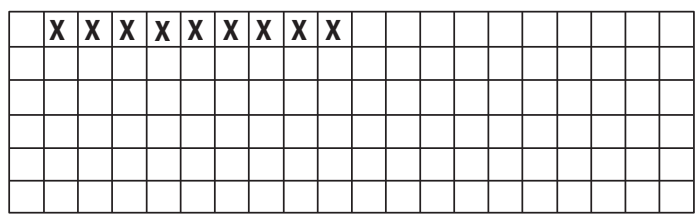

E. Cells that receive recharge FIXED_CELL option and recharge applied to all cells that user thought would receive recharge

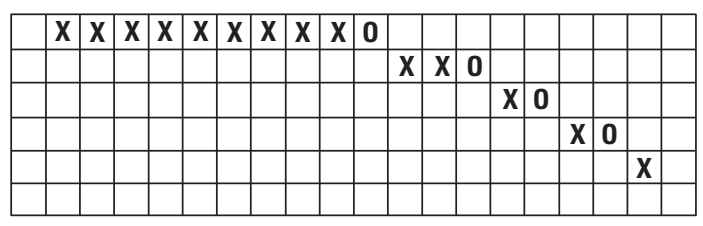

\section{EXPLANATION}

\begin{tabular}{|c|c|}
\hline 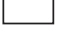 & Variable head \\
\hline & Constant head \\
\hline & Inactive \\
\hline $\mathbf{x}$ & $\begin{array}{l}\text { Cell that receives } \\
\text { recharge }\end{array}$ \\
\hline 0 & $\begin{array}{l}\text { Inactive cell specified by user } \\
\text { to receive recharge }\end{array}$ \\
\hline
\end{tabular}

Figure 6-2. Diagram showing the cells receiving recharge under the default option, which is based on applying recharge to the highest active cell, and the cells receiving recharge under the FIXED_CELL option. These options are available in the Recharge Package. Figure modified from Harbaugh (2005). 
Figure 6-2E illustrates the recharge distribution if the FIXED_CELL option is used, assuming that the user specifies recharge cells prior to the simulation on the basis of an estimated water-table position, which differs slightly from the computed water table obtained in the simulation process. Four of the cells that the user had designated as recharge cells have converted to a no-flow condition and, thus, receive no recharge.

For the typical situation of recharge from precipitation, the default option is the easiest to use. The model user does not have to be concerned about determining which is the highest variable-head cell in a vertical column because the program automatically determines this throughout the simulation. The FIXED_CELL option, however, can be useful in situations where recharge should not pass through the no-flow cells in layer 1. For example, some cells may be designated as no-flow cells because they are impermeable. Any recharge specified for those cells should not pass into layer 2. Alternately, the default option could still be used in this situation by specifying that the recharge rate is zero at the impermeable cells. Similarly, the FIXED_CELL option may be useful when layers other than layer 1 have outcrop areas and when recharge to the specified layers should not penetrate through no-flow cells to a lower layer.

The default recharge option has an additional complication for certain types of grids specified using the DISU Package. When the DISU Package is used, a cell may have more than one cell underlying it. If the overlying cell were to become inactive, there is no straightforward method for determining how to apportion the recharge flow to the underlying cells. In this case, the approach described by Panday and others (2013) is used. The entire recharge flow rate is assigned to the first active cell encountered (determined by searching through the underlying cell numbers from the lowest number to the highest number). In this manner, the total recharge flow rate is conserved; however, the spatial distribution of the applied recharge may not be maintained as layers become dry or wet during a simulation.

These issues of determining the highest active cell do not apply when the Newton-Raphson Formulation is used. In that case, all cells are considered active, even if their simulated head is below the cell bottom. In this case, recharge can simply be applied to the top of the model (with or without the FIXED_CELL option). The

Newton-Raphson formulation will automatically pass this recharge down to the cell containing the water table. For the example shown, the Newton-Raphson formulation would result in the recharge distribution shown in $6-2 C$.

The RCH Package can be used to simulate recharge from sources other than precipitation-for example, artificial recharge. Discharge can also be simulated using the RCH Package by specifying negative values of the recharge flux. If the ability to apply recharge to more than one cell in a vertical column of cells is required, then the Well Package, which allows recharge or discharge to be specified at any model cell, can be used.

\section{Standard Formulation}

In the standard formulation, the recharge flow rate $\left(Q R_{n b}\right)$ associated with a given horizontal cell $(n)$ that is determined by the recharge option is subtracted from the right-hand side of equation 2-24. Because recharge, as defined, is independent of aquifer head, nothing is added to the left-hand side of equation 2-24.

\section{Newton-Raphson Formulation}

The Newton-Raphson formulation for the recharge package is identical to the standard formulation, as the derivative of a specified flow boundary is zero. As a result, the left- and right-hand sides of equation 2-24 are not adjusted during the Newton-Raphson formulation. 


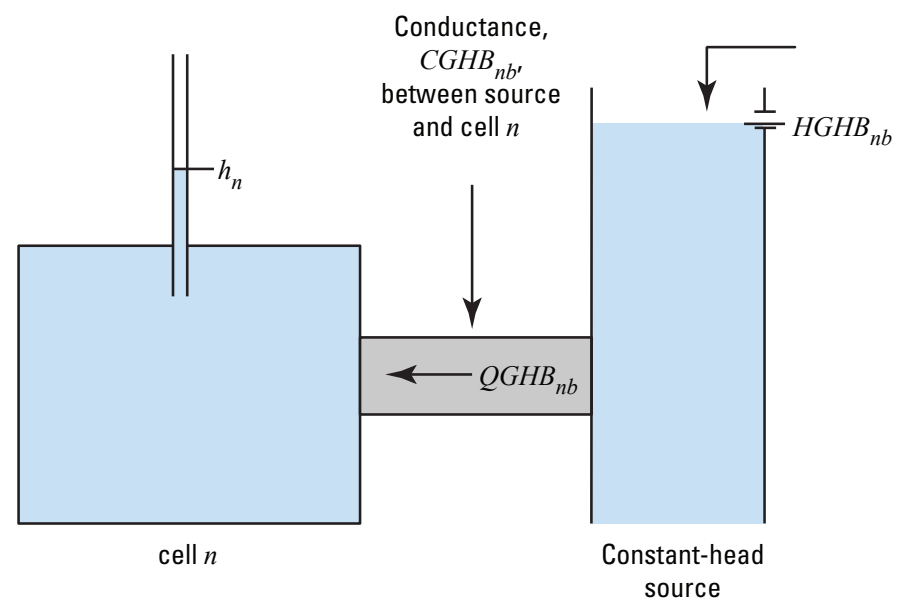

Figure 6-3. Diagram illustrating principle of the General-Head Boundary Package. Figure modified from Harbaugh (2005).

\section{General-Head Boundary Package}

The function of the General-Head Boundary (GHB) Package is to simulate flow into or out of a cell ( $n$ ), from an external source in proportion to the difference between the head in the cell and the head assigned to the external source. The constant of proportionality is called the boundary conductance. Thus, a linear relation between flow into the cell and head in the cell is established, as shown in the following equation:

$$
Q G H B_{n b}=C G H B_{n b}\left(H G H B_{n b}-h_{n}\right),
$$

where $Q G H B_{n b}$ is the flow into cell $n$ from the boundary expressed as a fluid volume per unit time $\left(\mathrm{L}^{3} \mathrm{~T}^{-1}\right)$, $C G H B_{n b}$ is the boundary conductance $\left(\mathrm{L}^{2} \mathrm{~T}^{-1}\right)$, and $H G H B_{n b}$ is the head assigned to the boundary condition (L).

The relation between cell $n$ and the external source is shown schematically in figure 6-3. The constanthead source is represented by the apparatus on the right in figure 6-3, which holds the source head at the level $H G H B_{n b}$, regardless of other factors; the link between the source and cell $n$ is represented by the block of porous material having conductance $C G H B_{n b}$. Note that figure 6-3 shows no mechanism to limit flow in either direction as $h_{n}$ rises or falls.

A graph of inflow from a general-head boundary and head in the cell containing the boundary as given by equation 6-21 is shown in figure 6-4. The GHB Package provides no limiting value of flow to bound the linear function in either direction; as the difference between the head in the cell containing the boundary and the source head increases, flow into or out of the cell continues to increase without limit. Accordingly, the GHB Package must be used with care to ensure that unrealistic flows into or out of the system do not develop during the course of simulation. The GHB Package is included in MODFLOW 6 without a specific real-world conceptualization in mind. The River, Drain, and Evapotranspiration Packages documented in the following sections of this chapter incorporate the same head-dependent form, except that they limit the flow to simulate the conceptualization of specific hydrologic features.

\section{Standard Formulation}

As part of the standard formulation, $C G H B_{n b}$ for each general-head boundary condition is subtracted from the diagonal of cell $n$ of equation 2-24. The product of $C G H B_{n b}$ and $H G H B_{n b}$ is also subtracted from 


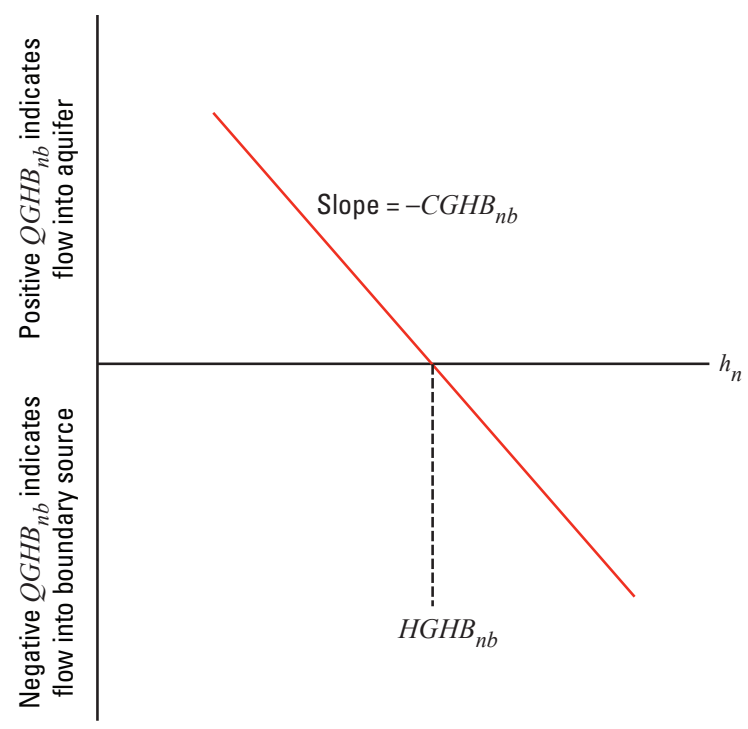

Figure 6-4. Graph showing $Q G H B$ flow for a general-head boundary as a function of head, $h$, in cell $n$, where $H G H B$ is the source head. Figure modified from Harbaugh (2005).

the right-hand side of equation 2-24 for the cell associated with every general-head boundary condition during the standard formulation.

\section{Newton-Raphson Formulation}

The Newton-Raphson formulation for the general-head boundary package is identical to the standard formulation, as the derivative of a linear head-dependent boundary is equal to the boundary conductance (eq. 64). As a result, the left- and right-hand sides of equation 2-24 are not adjusted during the Newton-Raphson formulation for the GHB Package. 


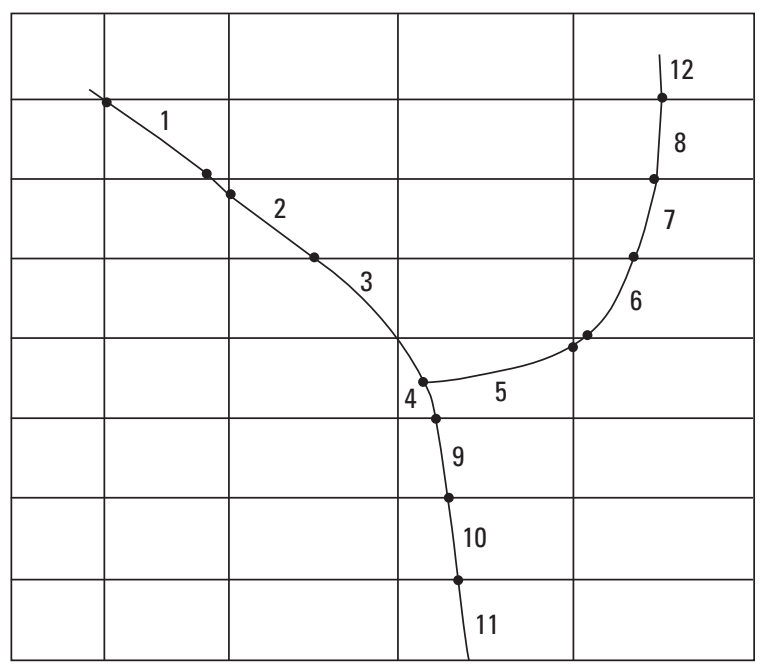

Figure 6-5. Diagram showing discretization of two rivers into reaches. Several short reaches are ignored. Figure modified from Harbaugh (2005).

\section{River Package}

Rivers and streams contribute water to or drain water from the groundwater system, depending on the head gradient between the river and the groundwater regime. The purpose of the River (RIV) Package is to simulate the effects of flow between surface-water features and groundwater systems. To accomplish this purpose, terms representing seepage to or from the surface features must be added to the groundwater flow equation for each cell affected by the seepage.

Figure 6-5 shows two rivers divided into reaches so that each reach is completely contained in a single cell. River-aquifer seepage is simulated between each reach and the model cell that contains that reach. Conceptually, a river boundary is located at the centroid of the model cell. The RIV Package does not simulate surface-water flow in the river-only the river/aquifer seepage. (If flow within the river or stream is of importance, then the Streamflow Routine Package, described in the next chapter, should be considered.) Accordingly, the order of numbering for reaches has no impact on calculations in the RIV Package. River seepage is independently simulated for each river reach.

The cross section in figure 6-6 shows a single cell containing a river reach. In the conceptualized system (fig. 6-6A), the open water of a river is separated from the groundwater system by a layer of low-permeability riverbed material. Figure 6-6B shows an idealization of this system in which the river-aquifer interconnection is represented as a simple conductance through which one-dimensional flow occurs.

In this example, the assumption is made that measurable head losses between the river and the aquifer are limited to those across the riverbed layer itself; that is, that no substantial head loss occurs between the bottom of the riverbed layer and the point represented by the underlying model node. Furthermore, an assumption is made that the underlying model cell remains fully saturated; that is, the water level does not drop below the bottom of the riverbed layer. Under these assumptions, flow between the river and the groundwater system for reach $n b$ is given by

$$
Q R I V_{n b}=C R I V_{n b}\left(H R I V_{n b}-h_{n}\right),
$$



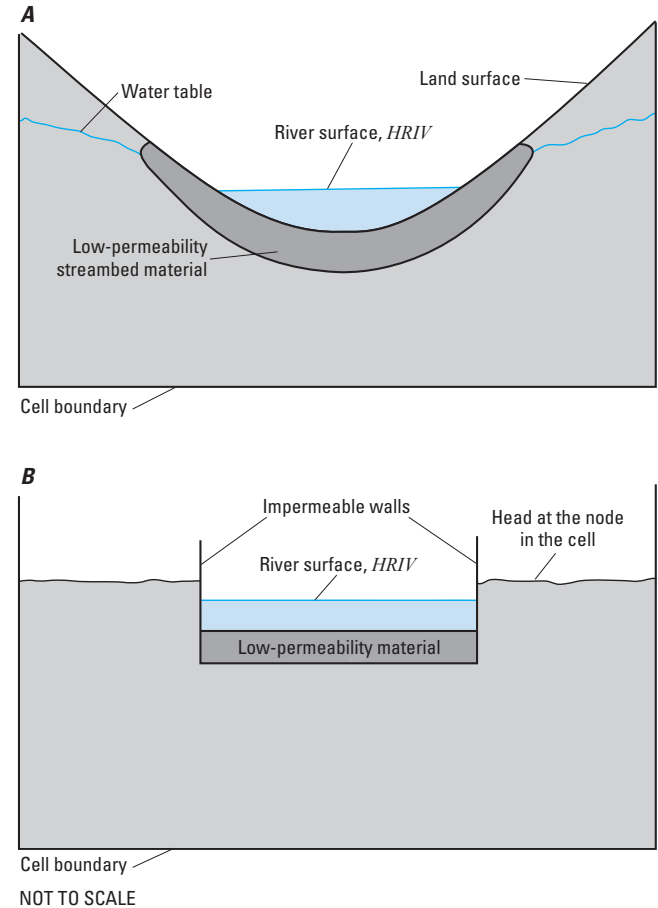

Figure 6-6. Diagram showing the interaction between a river and the underlying aquifer. $A$, cross section of an aquifer containing a river, and $B$, conceptual representation of river-aquifer interconnection in a simulation. Figure modified from Harbaugh (2005). 


\section{6-16 Documentation for the MODFLOW 6 Groundwater Flow Model}

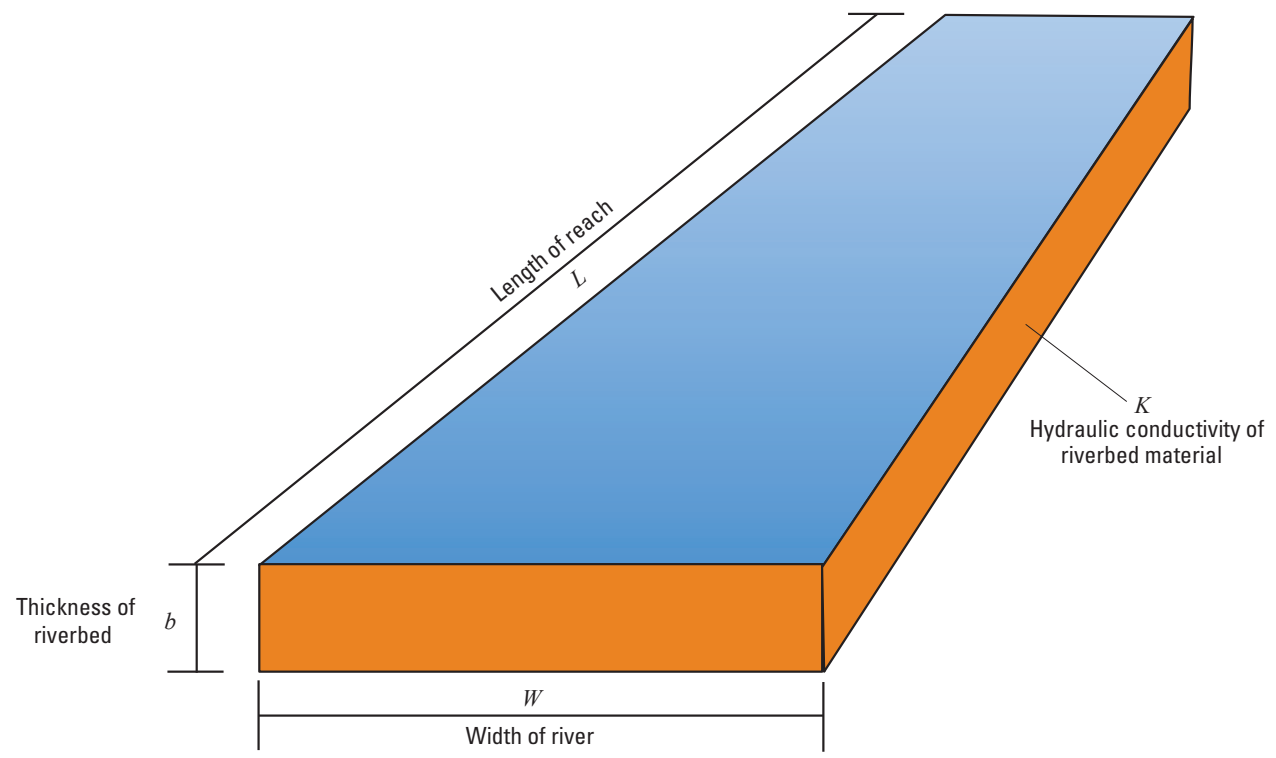

Figure 6-7. Diagram showing an idealized case of low-permeability riverbed sediments within an individual model cell. Figure modified from Harbaugh (2005).

where $Q R I V_{n b}$ is the flow between the river and the aquifer, taken as positive if it is directed into the aquifer $\left(\mathrm{L}^{3} \mathrm{~T}^{-1}\right) ; H R I V_{n b}$ is the water level (stage) in the river $(\mathrm{L}) ; C R I V_{n b}$ is the hydraulic conductance of the riveraquifer interconnection $\left(\mathrm{L}^{2} \mathrm{~T}^{-1}\right)$; and $h_{n}$ is the head at the node in the cell underlying the river reach (L).

Figure 6-7 shows an isolated view of the idealized riverbed conductance of figure 6-6B as it crosses an individual cell. The length $\left(L_{n b}\right)$ of the conductance block is the length of the river as it crosses the cell; the width $\left(W_{n b}\right)$ is the river width; the distance of flow is taken as the thickness $\left(b_{n b}\right)$ of the riverbed layer; and $K_{n b}$ is the hydraulic conductivity of the riverbed material. The conductance can then be computed as

$$
C R I V_{n b}=\frac{K_{n b} L_{n b} W_{n b}}{b_{n b}}
$$

Equation 6-23 normally provides an acceptable approximation of river-aquifer interaction over a certain range of aquifer head values. In most cases, however, if water levels in the aquifer fall below a certain point, seepage from the river ceases to depend on head in the aquifer. This can be visualized by returning to the concept of a discrete riverbed layer. Figure 6-8A shows the situation described by equation 6-23; the water level in the aquifer is above the bottom of the riverbed layer, and flow through that layer is proportional to the head difference between the river and the aquifer. In figure $6-8 B$, the water level in the aquifer has fallen below the bottom of the riverbed layer, leaving an unsaturated interval beneath that layer; if it is assumed that the riverbed layer itself remains saturated, the head at its base will simply be the elevation at that point. If this elevation is designated $R B O T_{n b}$, the flow through the riverbed layer is given by

$$
Q R I V_{n b}=C R I V_{n b}\left(H R I V_{n b}-R B O T_{n b}\right),
$$

where $Q R I V_{n b}, C R I V_{n b}$, and $H R I V_{n b}$ are defined the same as for equations 6-22 and 6-23. Obviously, further declines in head below $R B O T_{n b}$ produce no increase in flow through the riverbed layer; the flow simply retains the constant value given by equation 6-24, as long as head remains below $R B O T_{n b}$. Implied in this method of calculating flow to the aquifer when the water level in the cell is below the river bottom is that flow across the riverbed is translated directly to the water table without delay, and that the flow rate does not exceed 


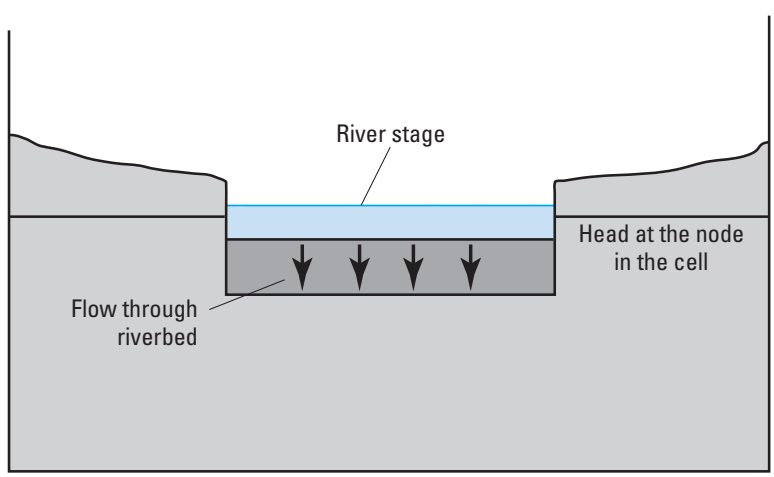

$A$. Head at the bottom of the riverbed is equal to the head at the node in the cell.

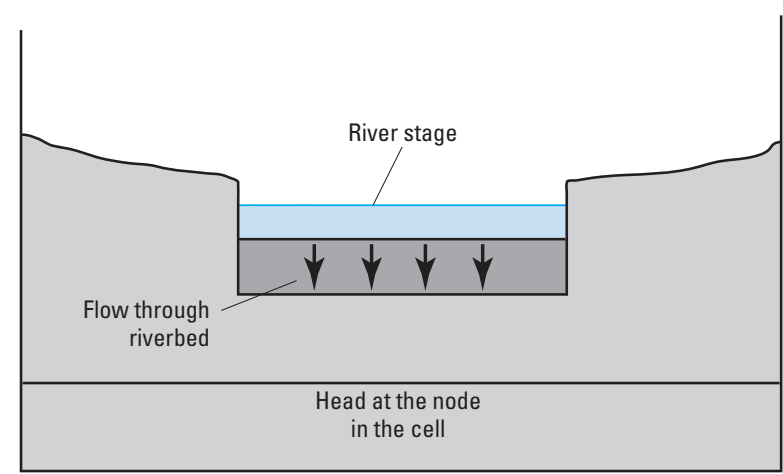

$B$. Head at the bottom of the riverbed is equal to elevation of bottom of riverbed.

NOT TO SCALE

Figure 6-8. Diagram of cross sections showing the relation between head at the bottom of the riverbed layer and head in the cell. Head in the cell is equal to the water-table elevation. Figure modified from Harbaugh (2005).

the saturated hydraulic conductivity of the deposits or rocks that occupy the interval between the riverbed and water table. Such assumptions generally are reasonable when the unsaturated interval is thin or when long periods of steady (constant) flow are modeled, and when the hydraulic gradient across the riverbed does not greatly exceed one.

MODFLOW 6 uses these concepts in simulating river-aquifer interaction; that is, flow between a river and a node $n$ is simulated according to the following equation set:

$$
\begin{array}{lll}
Q R I V_{n b}=C R I V_{n b}\left(H R I V_{n b}-h_{n}\right), & & h_{n}>R B O T_{n b} \\
Q R I V_{n b}=C R I V_{n b}\left(H R I V_{n b}-R B O T_{n b}\right), & & h_{n} \leq R B O T_{n b} .
\end{array}
$$

Figure 6-9 shows a graph of flow from a river reach as a function of the head, $h$, in the cell containing the reach, as calculated using equation 6-25. Flow is zero when $h$ is equal to the water level in the river, $H R I V$. For values of $h$ higher than $H R I V$, flow is into the river, represented as a negative inflow to the aquifer. For values of $h$ lower than $H R I V$, flow is positive into the aquifer. This positive flow increases linearly as $h$ decreases, until $h$ reaches $R B O T$; thereafter, the flow remains constant. Thus, the RIV Package is mathematically identical to the GHB Package as long as $h$ is greater than $R B O T$.

The conceptualization of river-aquifer interaction used here assumes that this interaction is independent of the location of the river reach within the cell, and that the level of water in the river is uniform over the reach and constant over each stress period. The latter assumption implies that conditions of flow in the river do not 


\section{6-18 Documentation for the MODFLOW 6 Groundwater Flow Model}

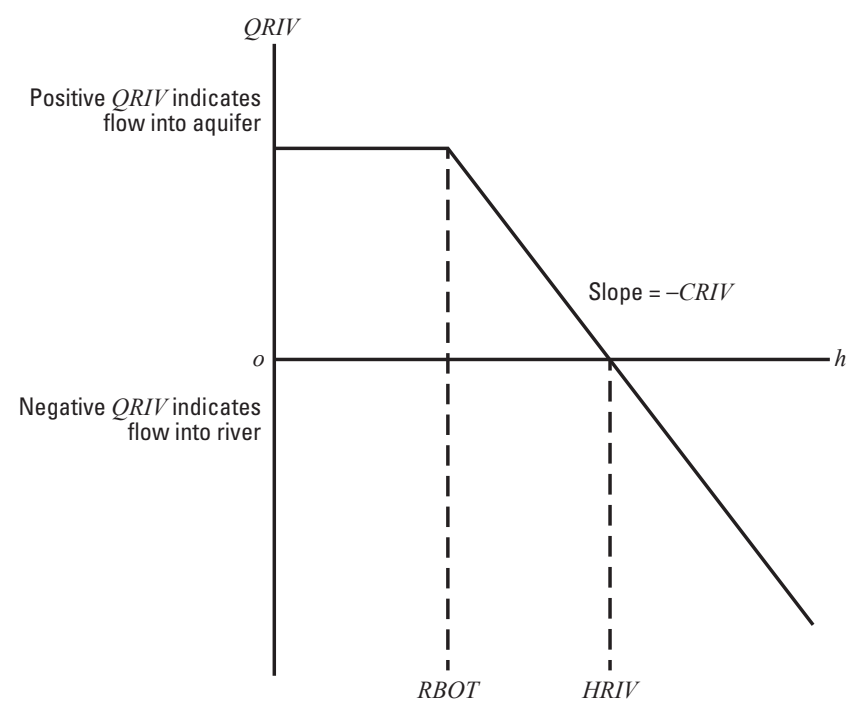

Figure 6-9. Graph showing $Q R I V$ as a function of head, $h$, in the cell, where $R B O T$ is the elevation of the bottom of the riverbed and $H R I V$ is the head in the river. Figure modified from Harbaugh (2005).

vary substantially during the stress period. For example, the river does not go dry or overflow its banks, or such events are of such short duration as to have no effect on river-aquifer interaction.

If the assumption that all substantial head loss occurs across a discrete, rectangular riverbed layer is satisfied, then the application of equations 6-23 and 6-25 is straightforward. The idealized riverbed conductance can be further generalized to include a variable width and a meandering river segment. In this case, the length would be the total length of the river reach in the cell, and the width would be the average width along the length. If reliable field measurements of river seepage and associated head difference are available, they may be used to calculate an effective conductance. Otherwise, a conductance value must be chosen more or less arbitrarily and adjusted during model calibration, as discussed in the next paragraph.

In general, the flow between the river and aquifer is a three-dimensional process, and its representation through a single conductance term and riverbed elevation is only approximate in situations in which the riverbed is much different from the idealized confining layer. For example, the bed may be discontinuous, multiple zones of low conductivity may be below the river, or perhaps no discrete riverbed layer can be identified. The user should recognize that use of a single conductance term to account for the resulting threedimensional flow process is inherently an empirical exercise, and that adjustment during calibration is almost always required. Adjustment through calibration is not always straightforward, however, because at relatively high conductance values, the model may be insensitive to changes. When this occurs, most of the head loss is likely within the aquifer itself, and the head loss through the riverbed does not have much effect on leakage. Certain rules can be formulated, however, to guide the initial choice of conductance in these situations. For example, the area through which flow occurs should still be viewed as the product of the total length of the river reach in the cell and the average width; the assumed distance of flow should not exceed the vertical interval between the riverbed and node $n$; and, if distinct layers can be recognized within this interval, these layers should normally be treated as conductances in series in formulating an equivalent conductance.

The application of equation 6-23 is the most difficult in situations where a discrete riverbed does not exist. In this case, equation 6-23 should be applied as an approximation. The following is a simple justification for applying the general relation of equation 6-25 in cases of no distinct riverbed. Although a strictly linear relation between head and seepage may not exist, the seepage into the river will increase as aquifer head increases above the river stage. Likewise, when the river is losing, seepage from the river will increase as head drops below the river stage. Thus, choosing a value for $C R I V$ that will approximate the seepage as represented by equation 6-25a should be possible, although the value of $C R I V$ should probably vary with aquifer head. The 


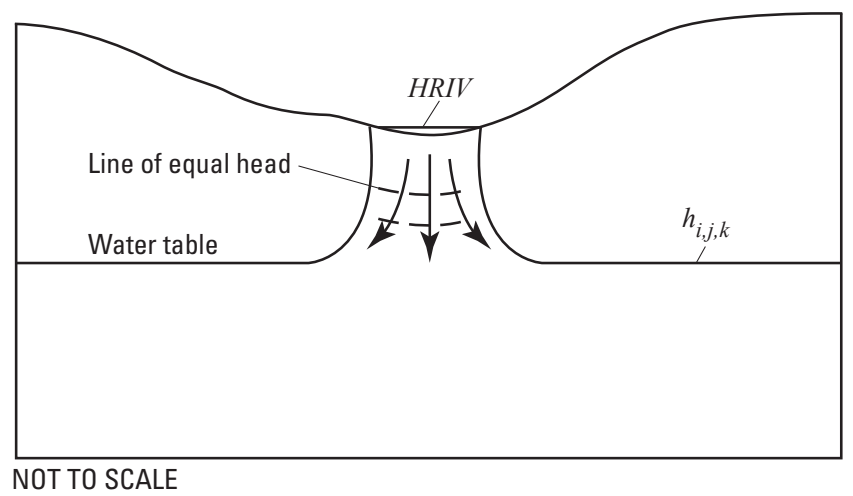

Figure 6-10. Diagram showing seepage from a river at unit hydraulic gradient. Figure modified from Harbaugh (2005).

existence of a depth below the river for which a further decrease in water level will not cause additional seepage also seems reasonable, and thus equation 6-25a would apply. This situation is conceptualized in figure 610 , where the water table has fallen substantially below the river and only a narrow saturated connection exists between the river and the regional water table. When the water table drops, as shown in figure 6-10, a region in the saturated column will form in which the head gradient above the water table is nearly 1 . Further lowering of the water table will not increase the gradient, and therefore the seepage should not increase. A break in saturation also will occur if the water table lowers enough, but leakage will not substantially increase once the head drops low enough to cause the region of unit gradient.

Unfortunately, the above argument that equation 6-25 can be qualitatively applied in the situation of no distinct riverbed does not provide much guidance on how to determine appropriate values for $C R I V_{n b}$ and $R B O T_{n b}$. One approach is to assume that the maximum seepage from the stream is the seepage in the aquifer in a column of water in which unity head gradient occurs. Darcy's law can be applied to compute the vertical flow, $Q \max$, through the water column. If the water column is assumed to have the same horizontal dimensions as the stream channel, $L_{n b}$ and $W_{n b}$, then the result is

$$
\operatorname{Qmax}_{n b}=K_{n} L_{n b} W_{n b} \frac{d h}{d l}
$$

where $K_{n}$ is the aquifer hydraulic conductivity in cell $n\left(\mathrm{LT}^{-1}\right)$ and $\frac{d h}{d l}$ is the head gradient through the water column (dimensionless). The head gradient is 1, so $\operatorname{Qmax}_{n b}=K_{n} L_{n b} W_{n b}$. Equation 6-22 can then be used to compute the conductance that will produce $Q \max$ when aquifer head has a specified value of $h_{n}$. If $h_{n}$ is replaced by $R B O T_{n b}$, then $C R I V_{n b}$ is

$$
C R I V_{n b}=\frac{K_{n} L_{n b} W_{n b}}{H R I V_{n b}-R B O T_{n b}} .
$$

In this situation, rather than being a distinct riverbed-bottom elevation, $R B O T_{n b}$ is the value of aquifer head at which the seepage from the river will equal $Q \max$. Seepage will not increase if the aquifer head goes lower. Regardless of the approach used to determine these values initially, some evaluation or adjustment is essential during the calibration process.

\section{Standard Formulation}

At the start of each iteration, terms representing river seepage are added to the flow equation for each cell containing a river reach. The choice of which river seepage equation to use, equation 6-25a or equation 6- 


\section{6-20 Documentation for the MODFLOW 6 Groundwater Flow Model}

$25 \mathrm{~b}$, is made by comparing the most recent value of head at the cell to the value of $R B O T_{n b}$ for the reach. Because this process is done at the start of each iteration, the most current value of head $\left(h_{n}\right)$ is the value from the previous iteration. Thus, the check for which river seepage equation to use lags behind the seepage calculations by one iteration. If equation 6-25a is selected, then the term $C R I V_{n b}$ is added to the term $H C O F_{n}$ and the term $C R I V_{n b} \times H R I V_{n b}$ is subtracted from $R H S_{n}$. If equation 6-25b is selected, then the term $C R I V_{n b}\left(H R I V_{n b}-R B O T_{n b}\right)$ is subtracted from $R H S_{n}$.

\section{Newton-Raphson Formulation}

The RIV Package boundaries can transition from head-dependent to specified flow when $h_{n}<R B O T_{n b}$. The Newton-Raphson formulation of the RIV Package is identical to the standard formulation, because the derivative of a head-dependent boundary with a constant conductance is equal to the conductance, and the derivative of a specified flow is 0 . 


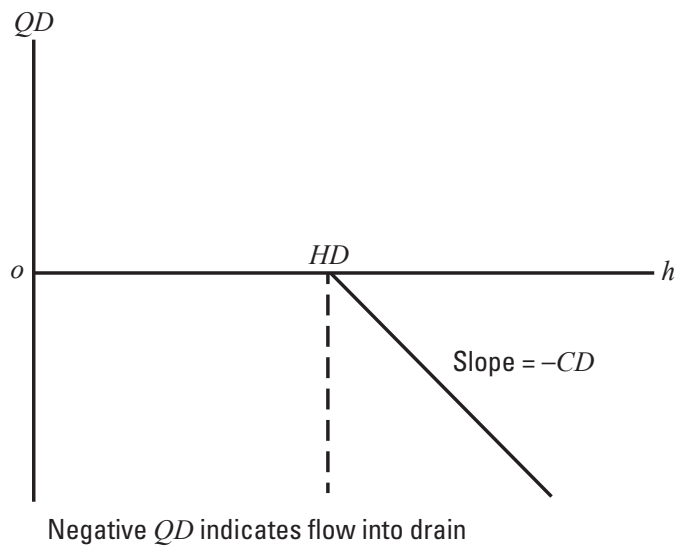

Figure 6-11. Graph showing $Q D R N$ as a function of head, $h$, in a cell, where elevation of the drain is $H D R N$ and the conductance is $C D R N$. Figure modified from Harbaugh (2005).

\section{Drain Package}

The Drain (DRN) Package is designed to simulate the effects of agricultural drains, springs, and other features that remove water from the aquifer at a rate proportional to the difference between the head in the aquifer and some fixed head or elevation, called the drain elevation, so long as the head in the aquifer is above that elevation. If, however, the aquifer head falls below the drain elevation, then the drain has no effect on the aquifer. The constant of proportionality is called the drain conductance. A mathematical statement of this situation is

$$
\begin{array}{lll}
\text { Qout }_{n b}=\operatorname{CDRN}_{n b}\left(h_{n}-\operatorname{HDRN}_{n b}\right), & & h_{n}>\operatorname{HDRN}_{n b} \\
\text { Qout }_{n b}=0, & & h_{n} \leq \operatorname{HDRN}_{n b},
\end{array}
$$

where Qout is the flow from the aquifer into the drain $\left(\mathrm{L}^{3} \mathrm{~T}^{-1}\right), C D R N_{n b}$ is the drain conductance $\left(\mathrm{L}^{2} \mathrm{~T}^{-1}\right)$, $H D R N_{n b}$ is the drain elevation (L), and $h_{n}$ is the head in the cell containing the drain (L).

Equation 6-28 is rewritten in terms of flow from the drain into the aquifer, $Q D R N$, which is the flow convention used throughout this report:

$$
\begin{aligned}
& Q D R N_{n b}=C D R N_{n b}\left(H D R N_{n b}-h_{n}\right), \quad h_{n}>H D R N_{n b} \\
& Q D R N_{n b}=0 \text {, } \\
& h_{n} \leq H D R N_{n b} \text {. }
\end{aligned}
$$

Thus, from the perspective of inflow to the model, drain flow is either negative or zero. For purposes of simulation, an assumption is made that each model drain represents the part of a physical drain that overlies a single model cell. Figure 6-11 shows a graph of flow from a drain and head in the cell containing the drain as defined by equation 6-29; the function is similar to that used for flow between a surface river and the aquifer (fig. 6-9), except that flow into the aquifer is excluded. With proper selection of coefficients (for example, setting $H R I V$ equal to $R B O T$ ), the River Package could in fact be used to perform the functions of the Drain Package.

Many physical conceptualizations can be approximated by equation 6-29. Figure 6-12A shows one conceptualization - a three-dimensional view of a buried drain tile (pipe containing slots) as used for agricultural drains. To justify representation by equation 6-29, the slope of the pipe is assumed to be great enough that once water enters the slots, it is carried away without filling the pipe. Accordingly, the drain runs only partially full, and the head representing the drain, $H D R N_{n d}$, is the average elevation of the slots. 


\section{6-22 Documentation for the MODFLOW 6 Groundwater Flow Model}

$\boldsymbol{A}$
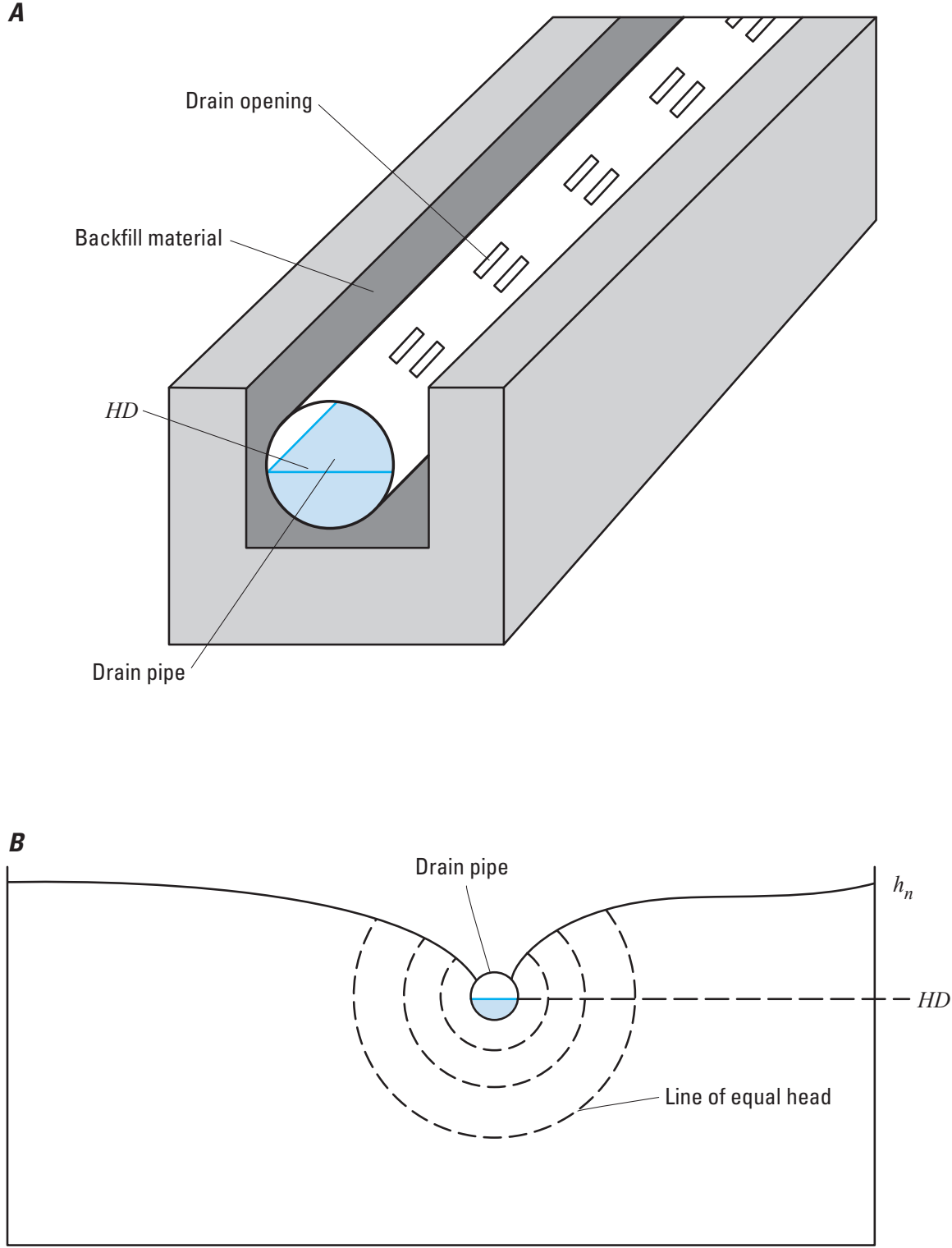

Figure 6-12. Diagrams showing drain conceptualization. $A$, factors affecting head loss in the immediate vicinity around a buried drain pipe in a backfilled ditch, and $B$, cross section through model cell illustrating head loss due to convergent flow into drain. Figure modified from Harbaugh (2005). 
Figure $6-12 B$ shows a cross section of cell $n$ that is traversed by the drain. The head computed by the model for cell $n\left(h_{n}\right)$ is actually an average value for the cell, and is normally assumed to prevail at some distance from the drain itself. $H D R N_{n b}$ prevails only locally at the drain pipe; it does not characterize the cell as a whole. Between the drain and the area in which head $h_{n}$ prevails, a radial or semiradial flow pattern exists in the vertical plane, normally characterized by progressively steeper head gradients as the drain is approached. The head loss within this converging flow pattern forms one part of the head difference, $h_{n}-H D R N_{n b}$. An additional component of head loss may occur in the immediate vicinity of the drain if the hydraulic conductivity in that region differs from the average value used for cell $n$, owing to the presence of foreign material around the drain pipe. Finally, head losses occur through the wall of a drain pipe, depending upon the number and size of the openings in the pipe, and the degree to which those openings may be blocked by chemical precipitates, plant roots, or other obstructions.

The three processes discussed above - convergent flow toward the drain, flow through material of differing conductivity in the vicinity of the drain, and flow through the wall of the drain - each generate head losses that may be assumed proportional to the discharge, $Q D R N$, into the drain from cell $n$. Because these head losses occur in series, the total head loss $H D R N_{n b}-h_{n}$ also may be taken as proportional to $Q D R N_{n b}$. This has been done in the method of simulation embodied in the Drain Package; that is, the drain function is assumed to be described by equation 6-29. The coefficient $C D R N_{n b}$ of equation 6-29 is a lumped (or equivalent) conductance describing all of the head loss between the drain and the region of cell $n$, in which the head $h_{n}$ can be assumed to prevail. Thus, $C D R N_{n b}$ depends on the characteristics of the convergent flow pattern toward the drain, as well as on the characteristics of the drain itself and its immediate environment.

One could attempt to calculate values for $C D R N_{n b}$ by developing approximate equations for conductance for the three flow processes, and then by calculating the equivalent series conductance. The conductance for each process would be based on a one-dimensional flow equation. The approaches vary substantially, depending on the specific drain system being simulated, so no general approach for calculating $C D R N_{n b}$ is presented here. Also, in most situations a specific approach would require detailed information that is not usually available, such as detailed head distribution around the drain, aquifer hydraulic conductivity near the drain, distribution of the fill material, hydraulic conductivity of fill material, number and size of the drain pipe openings, the amount of clogging materials, and the hydraulic conductivity of the clogging materials. In practice, calculating $C D R N_{n b}$ from measured values of $Q D R N_{n b}$ and $H D R N_{n b}-h_{n}$ using equation 6-29A is more common. If $H D R N_{n b}-h_{n}$ is not accurately known, $C D R N_{n b}$ is usually adjusted during model calibration in order to match measured values of $Q D R N_{n b}$ to model-calculated values.

Figure 6-13 shows two other real-world conceptualizations that can sometimes be simulated using equation 6-29. Figure 6-13A shows an open channel, which could be natural or man-made. If the channel carries water even when aquifer head is below the channel bed, then equation 6-29 presumably would not always apply because water could seep into the aquifer. In this case, the channel would be better simulated using the River (RIV) Package, as described earlier. But if the channel is dry unless groundwater is seeping into it, and there is no chance for this water to seep back into the aquifer, then the Drain Package could provide a reasonable representation. For example, the head waters of a river might be acceptably simulated with DRN, whereas the lower sections of a river might be simulated using the RIV Package.

Figure 6-13B shows a wetland, which can sometimes be conceptualized as functioning according to equation 6-29. If the wetland is wet primarily from groundwater seepage, then it will presumably dry up if the water table drops below the wetland. When wet, the conceptualized wetland water elevation is assumed to be fairly constant because excess groundwater seepage drains to a nearby river through a network of shallow channels or over the nearly flat surface. Seepage into the wetland is limited by low-conductivity sediments in the wetland. Under these conditions, equation 6-29 would be a reasonable approximation. Although one physical wetland might cover many cells, the part covered by each cell would be viewed as a separate drain in MODFLOW. The drain conductance for each drain would depend on the area of the cell covered by the wetland and the properties of the lower conductivity sediments in the wetland. 


\section{6-24 Documentation for the MODFLOW 6 Groundwater Flow Model}

A
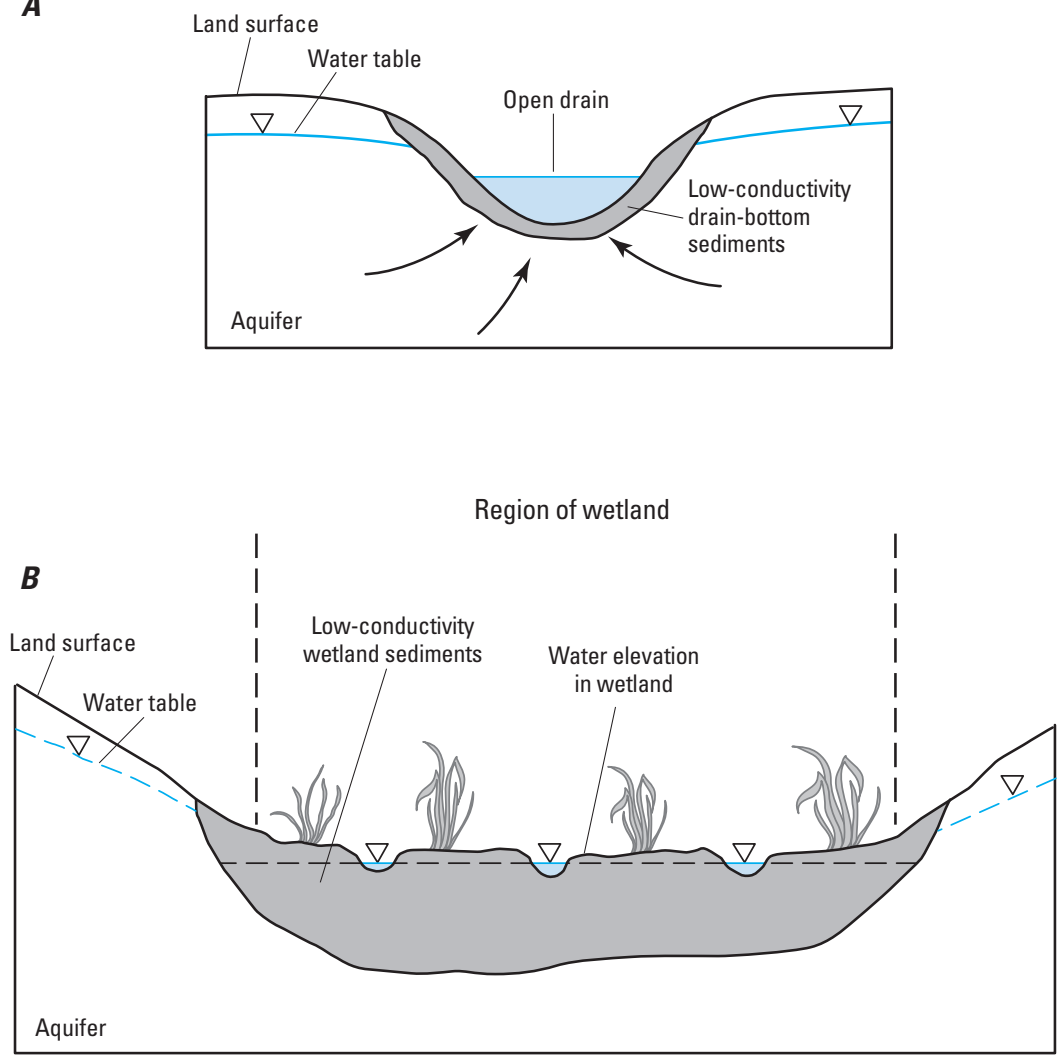

NOT TO SCALE

Figure 6-13. Diagram showing alternate drain conceptualizations. $A$, open drainage channel, and $B$, wetland. Figure modified from Harbaugh (2005). 


\section{Standard Formulation}

According to the sign convention in MODFLOW 6, $Q D R N_{n b}$ in equation 6-29 is defined as a flow into cell $n$ and must be added to the left side of equation 6-1 for each cell affected by a drain, provided the head $h_{n}$ is above the drain elevation. This is accomplished in the Drain Package by testing to determine whether head exceeds drain elevation, and if so, by adding the term $-C D R N_{n b}$ to $H C O F_{n}$ (equation 6-1) and subtracting the term $C D R N_{n b} \times H D R N_{n b}$ from $R H S_{n}$ as the matrix equations are assembled.

\section{Newton-Raphson Formulation}

The Drain Package boundaries can transition from head dependent to specified flow of zero when $h_{n}<$ $H D R N_{n b}$. The Newton-Raphson formulation of the Drain Package linear equation is identical to the standard formulation, as the derivative of a head-dependent boundary with a constant conductance is equal to the conductance, and the derivative of a specified flow (zero, in this case) is zero. 


\section{6-26 Documentation for the MODFLOW 6 Groundwater Flow Model}

\section{Evapotranspiration Package}

The Evapotranspiration (ET) Package simulates the effects of plant transpiration and direct evaporation in removing water from the saturated groundwater regime. The UZF Package described in the next chapter can be used to represent evaporation and transpiration in the unsaturated zone. The ET Package in MODFLOW 6 includes the capability to specify multiple linear ET segments, as implemented in the Segmented Evapotranspiration Package described by Banta (2000).

The approach for simulating ET is based on the following assumptions: (1) when the water table is at or above a specified elevation, termed the "ET surface" in this report, ET loss from the water table occurs at a fixed rate specified by the user; (2) when the depth of the water table below the ET surface elevation exceeds a specified interval, termed the "extinction depth" or "cutoff depth" in this report, ET from the water table ceases; and (3) between these limits, ET from the water table varies in a piecewise-linear fashion with watertable elevation. The interval between the ET surface and the extinction depth is referred to as the "variable interval."

In the ET Package, the relation of ET rate to head is conceptualized as a segmented line (a piecewiselinear function) in the variable interval. The segments that determine the shape of the function in the variable interval are defined by intermediate points where adjacent segments join. The ends of the segments at the top and bottom of the variable interval are defined by the ET surface and the extinction depth.

For the simplest case, where a single ET segment is used (equivalent to the EVT Package in MODFLOW2005), the ET rate is calculated as

$$
\begin{array}{lrr}
R E T_{n b}=E V T R_{n b}, & h_{n}>S U R F_{n b} & \text { (6-30a) } \\
R E T_{n b}=E V T R_{n b} \frac{h_{n}-\left(S U R F_{n b}-E X D P_{n b}\right)}{E X D P_{n b}}, & \left(S U R F_{n b}-E X D P_{n b}\right) \leq h_{n} \leq S U R F_{n b} & (6-30 \mathrm{~b}) \\
R E T_{n b}=0, & h_{n}<\left(S U R F_{n b}-E X D P_{n b}\right), & (6-30 \mathrm{c})
\end{array}
$$

where $R E T_{n b}$ is the rate of loss per unit surface area of water table due to ET, in units of volume of water per unit area per unit time $\left(\mathrm{LT}^{-1}\right) ; h_{n}$ is the head, or water-table elevation in cell $n$ from which the ET occurs (L); $E V T R_{n b}$ is the maximum possible value of $R E T\left(\mathrm{LT}^{-1}\right) ; S U R F_{n b}$ is the ET surface elevation, or the watertable elevation at which this maximum value of ET loss occurs (L); and $E X D P_{n b}$ is the cutoff or extinction depth (L), such that when the distance between $h_{n}$ and $S U R F_{n b}$ exceeds $E X D P_{n b}$, ET ceases.

In implementing the finite-difference approach, the volumetric rate of ET loss from a given cell is required. The volumetric ET loss is given as the product of the loss rate per unit area, and the horizontal surface area, $A_{n}$ of the cell $n$ from which the loss occurs, as in the following equation:

$$
Q E T_{n b}=R E T_{n b} M_{n b} A_{n}
$$

where $Q E T_{n b}$ is the ET loss, in volume of water per unit time $\left(\mathrm{L}^{3} \mathrm{~T}^{-1}\right)$, through the area $A_{n}$, and $M_{n b}$ is a multiplier (dimensionless) that can be used to scale $R E T_{n b}$. The multiplier can be used, for example, to scale ET data to account for vegetation and area factors; when combined with the capability to specify multiple ET packages per simulation or with list-based input of ET, this can be used to simulate the equivalent of the Riparian Evapotranspiration Package available for MODFLOW-2005 (Maddock and others, 2012).

If the maximum value of $Q E T_{n b}$ (corresponding to $E V T R_{n b}$ ) is designated $Q E T M_{n b}$, equation 6-30 for the simple single segment case can be expressed in terms of volumetric discharge as 


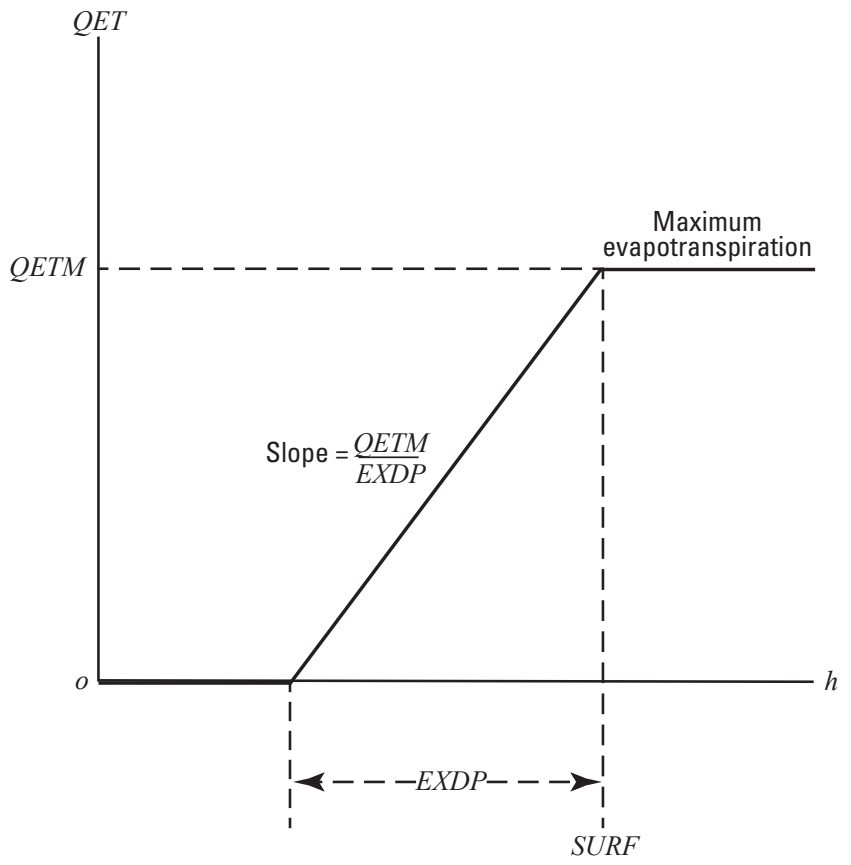

Figure 6-14. Graph of volumetric evapotranspiration (ET), $Q E T$, for a single ET segment, as a function of head, $h$, in a cell, where $E X D P$ is the cutoff depth and $S U R F$ is the ET surface elevation. Figure modified from Harbaugh (2005).

$$
\begin{array}{lc}
Q E T_{n b}=Q E T M_{n b}, & h_{n}>S U R F_{n b} \\
Q E T_{n b}=Q E T M_{n b} \frac{h_{n}-\left(S U R F_{n b}-E X D P_{n b}\right)}{E X D P_{n b}}, & \left(S U R F_{n b}-E X D P_{n b}\right) \leq h_{n} \leq S U R F_{n b} \\
Q E T_{n b}=0, & h_{n}<\left(S U R F_{n b}-E X D P_{n b}\right) .
\end{array}
$$

Figure 6-14 shows a graph of ET loss, QET, from a cell and head in the cell based on equation 6-32. Comparison of the ET function with the river or drain functions shows that the three are mathematically similar, except that the linear part of the ET function is bounded at both ends by constant values rather than only at one end. Note that $Q E T$ (eq. 6-32 and fig. 6-14) represents outflow from the groundwater system, which is counter to the convention in MODFLOW of representing stresses as inflow. QET was developed in terms of outflow because it can be conceptually confusing to view ET as a negative quantity.

For the case where there is more than one segment and $h_{n}$ is in the variable interval, $Q E T_{n b}$ is calculated using

$$
\begin{aligned}
Q E T_{n b}= & P_{E T M_{n b_{n s-1}} Q_{E T M_{n b}-}} \\
& \frac{P E T M_{n b_{n s-1}}-P E T M_{n b_{n s}}}{P X D P_{n b_{n s-1}}-P X D P_{n b_{n s}}} \frac{\operatorname{QETM}_{n b}}{E X D P_{n b}}\left(\left(S U R F_{n b}-h_{n}\right)-P X D P_{n s-1} E X D P_{n b}\right),
\end{aligned}
$$

where $n s$ is the segment number of the applicable segment and refers to the point at the bottom of the segment, $\operatorname{PETM}_{n b_{n s}}$ is a proportion (between zero and one) of the maximum ET rate $\left(Q E T M_{n b}\right)$ for segment $n s$, and 


\section{6-28 Documentation for the MODFLOW 6 Groundwater Flow Model}

$P X D P_{n b_{n s}}$ is a proportion (between 0.0 and 1.0) of the extinction depth $\left(E X D P_{n b}\right)$ for segment $n s$. When $n s$ is $1, n s-1$ refers to the upper end of the first segment, where $P X D P_{n b}=0.0$ and $P E T M_{n b}=1.0$. Similarly, for the last segment, $n s$ is the total number of segments, $P X D P_{n s}=1.0$, and $P E T M_{n s}=0.0$.

The number of intermediate points that must be defined is one less than the number of segments in the variable interval. For each intermediate point, two values, $P X D P_{n b_{n s}}$ and $P E T M_{n b_{n s}}$, are entered to define the point. Segments are numbered such that segment one is the segment with its upper endpoint at the ET surface, and segment numbers increase downward.

Figure 6-15A illustrates a possible function relating volumetric ET rate to head for a situation where three segments are used. The function in the variable interval is defined by the segments AB, BC, and CD. Points A and $\mathrm{D}$ are determined by the ET surface $\left(S U R F_{n b}\right)$, the product of the maximum ET flux rate $\left(Q E T M_{n b}\right)$, and the extinction depth $\left(E X D P_{n b}\right)$. Points $\mathrm{B}$ and $\mathrm{C}$ are intermediate segment endpoints. The user inputs $P X D P_{n b_{n s}}$ and $P E T M_{n b_{n s}}$ to define each intermediate endpoint. For ET boundary condition $n b$ corresponding to figure 6-15A, $P X D P_{B}$ equals $0.17, P E T M_{B}$ equals $0.56, P X D P_{C}$ equals 0.46 , and $P E T M_{C}$ equals 0.25 .

Figure 6-15B illustrates a situation where the maximum ET rate applies when the head in the cell is at some depth below the ET surface. The optional variable PETMO defines the proportion of the maximum ET rate that applies when the head is at or above the ET surface.

In some cases, a segmented relation between ET rate and head in one part of the model area and a simple linear relation in another part may be desired. The simple linear relation (equivalent to the EVT Package in MODFLOW-2005) can be modeled by specifying $P X D P_{n b_{n s}}$ and $P E T M_{n b_{n s}}$ such that $P E T M_{n b_{n s}}=(1-$ $\left.P X D P_{n b_{n s}}\right)$ for each intermediate endpoint.

Figures 6-14 and 6-15 show graphs of ET loss, QET, from a cell and head in the cell based on equations 6-32 and 6-33. Comparison of the ET function with the river or drain functions shows that the three are mathematically similar, except that the linear part(s) of the ET function is bounded at both ends by constant values, rather than only at one end. Note that $Q E T_{n b}$ (eqs. 6-32 and 6-33 and figs. 6-14 and 6-15) represents outflow from the groundwater system, which is counter to the convention in MODFLOW of representing stresses as inflow. $Q E T_{n b}$ was developed in terms of outflow because it can be conceptually confusing to view ET as a negative quantity.

ET is conceptualized as an areal phenomenon, like recharge simulated by the Recharge Package. The ET rate, $Q E T_{n b}$, is applied to the cell containing ET boundary condition $n b$. In the simplest situation, the top of the groundwater system will occur in the uppermost cell in a vertical column; however, the vertical position of the top of the system may vary with horizontal location and with time as the water table rises and falls. Two options for specifying the cell in each vertical column of cells from which ET is to be taken have been implemented as described below.

The cell within each vertical column to which the ET is applied is specified in the EVT Package input file. If the default option is specified, then ET is taken from the highest active cell. If the FIXED_CELL option is used and the specified cell is dry or a constant head, then ET is not taken. Potential ET values $\left(E V T R_{n b}\right)$ that are read into the model must be expressed in units that are consistent with the length and time units used to represent all other model data.

\section{Standard Formulation}

Equations 6-32 and 6-33 are rewritten in terms of inflow to a model cell, as is the convention in MODFLOW 6. Equation 6-32c is further rearranged algebraically for convenient implementation in the code. The resulting equations are 

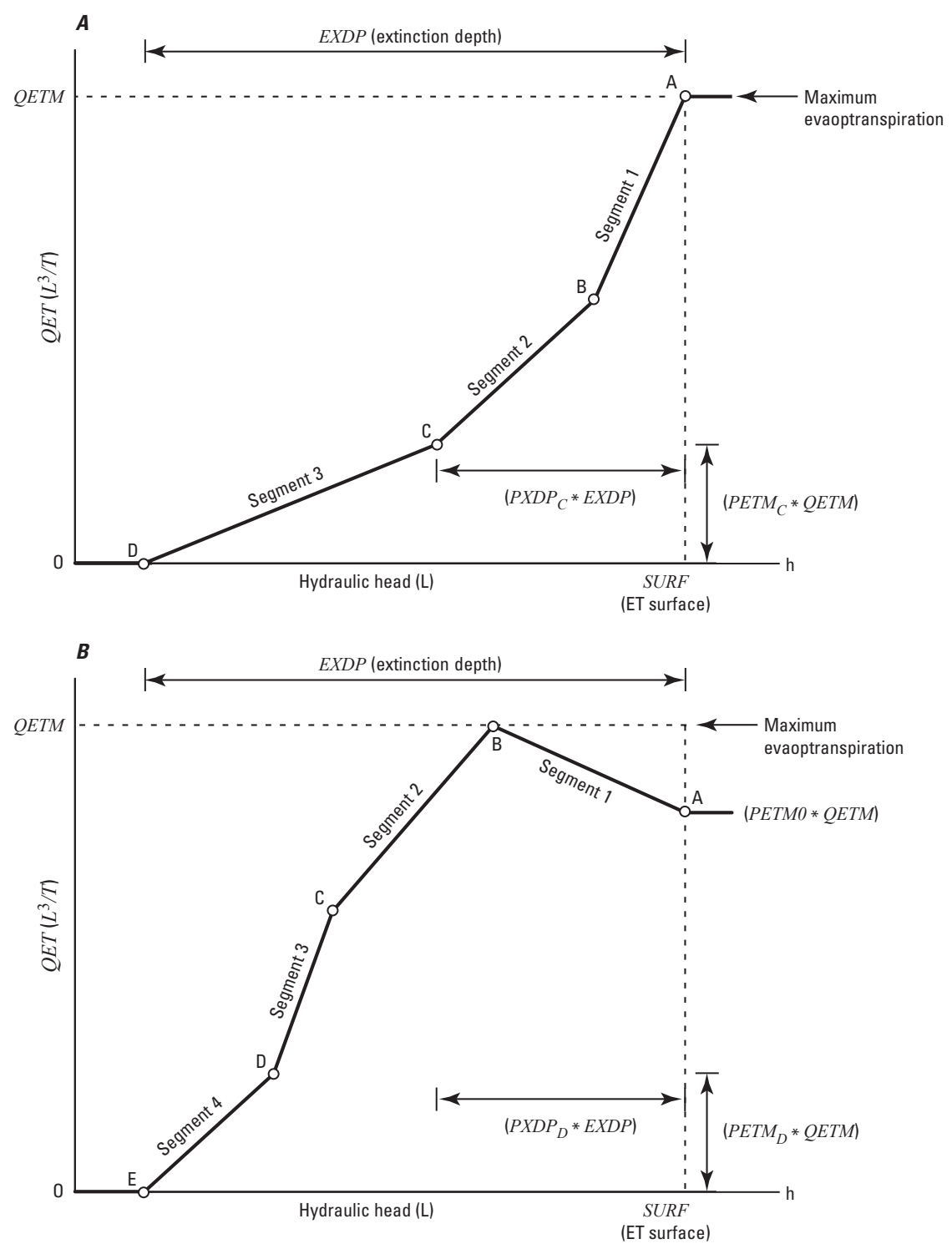

Figure 6-15. Graphs showing volumetric evapotranspiration (ET) rate, $Q E T$, as a function of head for two situations in which the function in the variable interval is defined using multiple segments. EXDP is the extinction depth, SURF is the ET surface, and QETM is the product of the maximum ET flux rate and the cell area. In $A$, the maximum ET rate applies at $S U R F$, and $P E T M_{C}$ and $P X D P_{C}$ define the location of point C. In $B$, the ET rate that applies at $S U R F$ is defined by the product of the optional variable $P E T M O$ and $Q E T M$. 


$$
\begin{aligned}
& \operatorname{QETin}_{n b}=-\operatorname{QETM}_{n b}, \\
& h_{n}>S U R F_{n b} \\
& \operatorname{QETin}_{n b}=-\frac{Q E T M_{n b}}{E X D P_{n b}} h_{n}-Q E T M_{n b}+\frac{Q E T M_{n b} S U R F_{n b}}{E X D P_{n b}}, \quad\left(S U R F_{n b}-E X D P_{n b}\right) \leq h_{n} \leq S U R F_{n b} \\
& \operatorname{QETin}_{n b}=0 \text {, } \\
& h_{n}<\left(S U R F_{n b}-E X D P_{n b}\right)
\end{aligned}
$$

For the case where there is more than one segment and $h_{n}$ is in the variable interval, QETin $_{n b}$ is calculated using

$$
\begin{aligned}
\operatorname{QETin}_{n b}=- & \frac{P E T M_{n b_{n s-1}}-P E T M_{n b_{n s}}}{P X D P_{n b_{n s-1}}-P X D P_{n b_{n s}}} \frac{\operatorname{QETM}_{n b}}{\operatorname{EXDP}_{n b}} h_{n}- \\
& \quad \operatorname{QETM}_{n b}\left[P E T M_{n b_{n s-1}}-\frac{P E T M_{n b_{n s-1}}-P E T M_{n b_{n s}}}{P X D P_{n b_{n s-1}}-P X D P_{n b_{n s}}}\left(\frac{S U R F_{n b}}{E X D P_{n b}}-P X D P_{n b_{n s-1}}\right)\right] .
\end{aligned}
$$

In terms of the expression $H C O F$ and $R H S$ of equation 6-1, ET is added to the flow equation as follows:

1. if $h_{n}>S U R F_{n b}, Q E T M_{n b}$ is added to $R H S_{n}$;

2. if $\left(S U R F_{n b}-E X D P_{n b}\right) \leq h_{n} \leq S U R F_{n b}$, the coefficient in front of $h_{n}$ in the first term on the righthand side of equations 6-34b or 6-35 is added to $\mathrm{HCOF}_{n}$ and the second and third terms on the righthand side of equation $6-34 \mathrm{~b}$ or the second term on the right-hand side of equation 6-35 is subtracted from $R H S_{n}$; and

3. if $h_{n}<\left(S U R F_{n b}-E X D P_{n b}\right), H C O F_{n}$ or $R H S_{n}$ are not changed.

The value of $S U R F_{n b}$, the water-table elevation at which ET is maximum (when PETMO is not specified), is sometimes assumed to be the land-surface elevation. However, the maximum ET usually occurs at some depth below land surface because plants can generally withdraw the maximum amount from groundwater when a fraction of the root zone is in contact with the water table; by specifying the optional variable PETM0, the modeler can simulate this situation. The cutoff or extinction depth, EXDP ${ }_{n b}$, is frequently assumed to be the distance from $S U R F_{n b}$ down to the bottom of the deepest roots. Considerable variation can be introduced by climatic factors and plant type.

\section{Newton-Raphson Formulation}

The ET Package calculations transition from head-dependent to specified flow when $h_{n}>S U R F_{n b}$ or when $h_{n}<E X D P_{n b}$. The Newton-Raphson formulation of the ET Package is identical to the standard formulation, because the derivative of a head-dependent boundary with a constant conductance-like term is equal to the conductance-like term, and the derivative of a specified flow is 0 . 
Chapter 6. Conceptualization and Implementation of Stress Packages 6-31
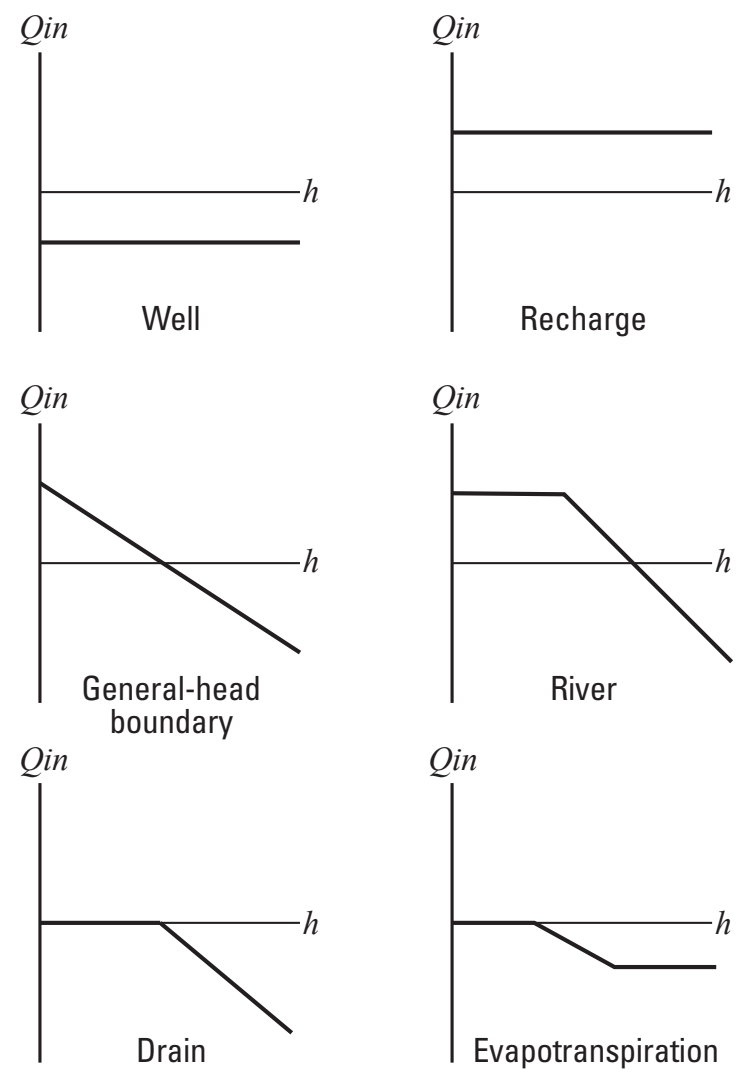

Figure 6-16. Diagram showing graphs of flow into or out of a model cell for the different stress packages. Figure modified from Harbaugh (2005).

\section{Summary of Stress Packages}

Figure 6-16 schematically shows flow into a model cell containing a stress, $\operatorname{Qin}$, and head in the cell, $h$, for all six stress packages documented in this chapter. These plots illustrate qualitatively the functional differences among the stress packages. Notice that the flows by the Well and Recharge Packages are independent of head in the model cell, whereas the flows calculated for the remaining packages are dependent on head in the model cell. The well inflow is negative, illustrating the typical situation of a pumping well, but any positive or negative value can be applied. Similarly, the recharge inflow is shown as positive, but negative values can be used as desired. The plot for ET has been reversed to show inflow to the model rather than outflow, as in figure 6-14. 


\section{Chapter 7. Conceptualization and Implementation of Advanced Stress Packages}

This chapter describes advanced packages for the Groundwater Flow (GWF) Model. These advanced packages include the Streamflow Routing (SFR) Package, the Lake (LAK) Package, the Multi-Aquifer Well (MAW) Package, and the Unsaturated Zone Flow (UZF) Package. A fifth package, called the Water Mover (MVR) Package is also described here. The MVR Package is designed to move water into the advanced packages. Water that is moved can come from other advanced packages or from groundwater discharge to the Well, General-Head Boundary, Drain, and River Packages.

\section{Streamflow Routing Package}

The Streamflow Routing (SFR) Package uses the continuity equation and assumption of piece-wise steady (nonchanging in discrete time periods), uniform (nonchanging in location), and constant-density streamflow, such that during all times, volumetric inflow and outflow rates are equal and no water is added to or removed from storage in the surface channels to route streamflow through a network of rectangular channels (which may include rivers, streams, canals, and ditches, and are referred to collectively as reaches in the remainder of the report). The network of channels may or may not be interconnected. Streamflow is always in the same direction along the channels, and is constant for each time step used in the GWF Model. If changes in stream channel storage during each time step used in the groundwater flow model are important, then more complicated, one-dimensional unsteady flow models may be required. The SFR Package for MODFLOW 6 calculates flow across the streambed on the basis of stream depth calculated (active reaches) or specified stream depth (simple routing option reaches) at the midpoint of a rectangular reach.

\section{Ordering and Numbering of Reaches}

The network in the SFR Package is divided into reaches. Previous versions of the SFR Package (for example, Niswonger and Prudic, 2005) discretized streams into a combination of segments (grouped based on geometry, boundary conditions, and connectivity) and segments (portions of stream segments associated with a particular cell), by default. In MODFLOW 6, segments have been eliminated and a stream reach is a section of a stream that is associated with a particular cell used to model groundwater flow. Each reach can be assigned (1) uniform rates of overland flow and precipitation to them; (2) uniform rates of evaporation from them; (3) uniform properties (for example, streambed elevation, thickness, and hydraulic conductivity, as well as stream depth and width); (4) tributary flows or specified inflow or outflow; and (5) diversions.

A simple example of a stream network superimposed on a rectilinear finite-difference grid of an aquifer that has three columns and three rows is shown in figure 7-1. Each cell is designated first by row number followed by column number (for example, cell $(1,2)$ is row 1 , column 2 ). In this example, there are six reaches. The first reach is in cell $(1,1)$; the second reach is in cell $(1,2)$; the third reach is in cell $(2,2)$; the fourth reach is in cell $(2,2)$; the fifth reach is in cell $(2,2)$; and the sixth reach is in cell $(3,2)$. A specified inflow is defined for the first reach and a specified inflow of zero is defined for reach 4 . Inflow to reach 4 occurs only when the head in the aquifer exceeds the elevation of the streambed. Inflow to reach 5 is the sum of outflow from reaches 3 and 4 .

More than one stream reach can be assigned to a model cell (figs. 7-1 and 7-2). Stream reaches may join together (fig. 7-2A) or parallel reaches can flow across a cell without joining (fig. 7-2B). When there is more than one stream reach in a model cell, the simulated groundwater head in the cell is used to calculate groundwater exchange for each of the stream reaches associated with the cell; the SFR Package maintains the accounting for all stream reaches contained in the cell. However, the SFR Package does not allow for one stream reach to be associated with more than one model cell (fig. 7-2C). 


\section{7-2 Documentation for the MODFLOW 6 Groundwater Flow Model}

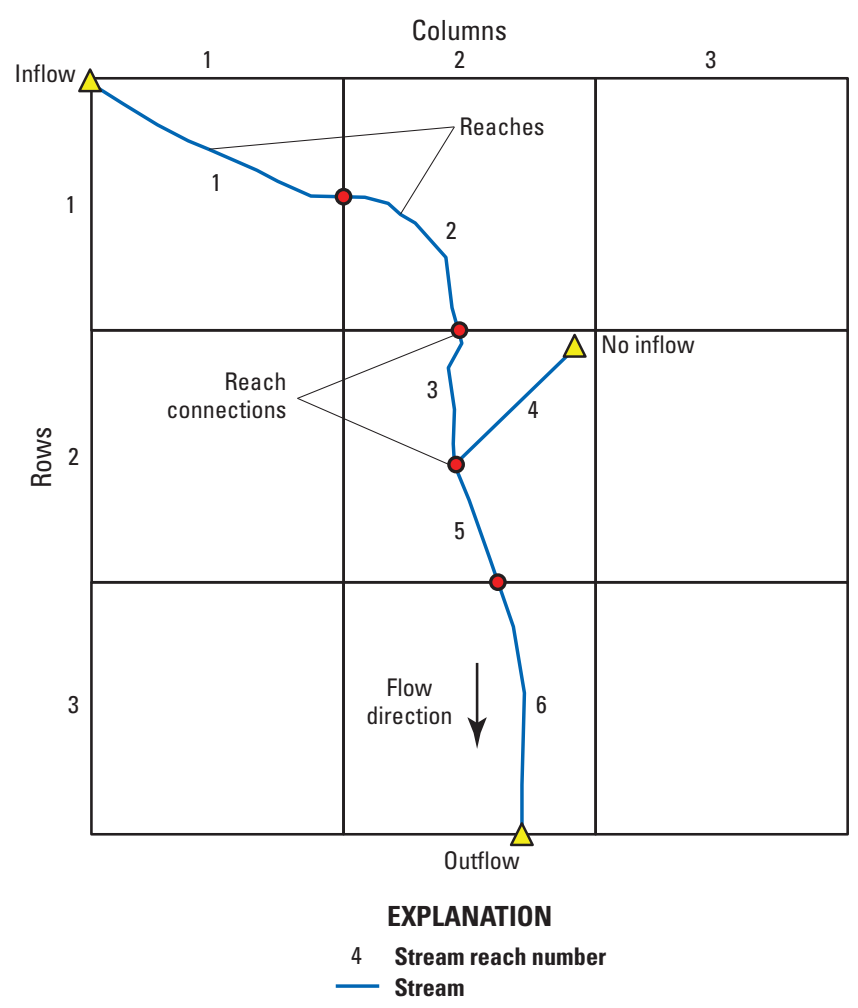

Figure 7-1. Diagram of a simple stream network having six reaches shown within a regular MODFLOW grid consisting of three rows and three columns. Figure modified from Prudic and others (2004).

The numbering of reaches is important. Reaches should be numbered sequentially from the farthest upstream reach to the last downstream reach. Multiple reaches may be joined as tributary streams. In most instances, the joining reaches will not be sequential. However, as long as the upstream reaches have a lower number than the downstream reaches, outflows from the upstream reaches will be added correctly as inflow to the downstream reach. If tributary reaches have higher numbers than the connecting downstream reaches, then outflows from the higher numbered tributary reaches will be from the previous iteration during the MODFLOW 6 solution of groundwater heads for each time step. As long as the difference in head between iterations is small (a small specified closure criteria), the error caused by adding outflow from the previous iteration in the higher numbered tributary reaches to the lower numbered connecting reaches will be small. An example of a rectilinear finite-difference grid of a hypothetical aquifer connected to streams is shown in figure 7-3.

For each reach, the basic information of the layer, row, and column of the cell that corresponds to each reach and the length of the stream reach in the cell is read only once for a model simulation. It is assumed that the locations of rivers, streams, canals, and ditches remain fixed during the simulation.

\section{Reach-Aquifer Connection}

Conceptually, the method of computing flow between reaches and aquifers is the same as that used for the River Package. Flow between a stream reach and a GWF cell is computed using Darcy's Law and assuming uniform flow between a stream and aquifer over a given reach and corresponding volume of aquifer. This flow, $Q S F R_{n b}$, is computed as

$$
Q S F R_{n b}=S_{S F R, n b}^{*} \frac{K_{n b} L_{n b} W_{n b}}{b_{n b}}\left(H S F R_{n b}-h_{n}\right),
$$


where $S_{S F R, n b}^{*}$ is a cubic saturation function (-), $K_{n b}$ is the hydraulic conductivity of the sediments underlying the reach $\left(\mathrm{LT}^{-1}\right), L_{n b}$ is the length of the reach in the cell $(\mathrm{L}), W_{n b}$ is the width of the reach $(\mathrm{L}), b_{n b}$ is the thickness of the reach streambed sediments (L), $H S F R_{n b}$ is the simulated stage in the reach (L), and $h_{n}$ is the head at the node underlying the reach $(\mathrm{L}) . S_{S F R, n b}^{*}$ varies from 0 to 1 for stream depths ranging from 0 to a small value above the top of the streambed, respectively.

In this approach, transient leakage across the streambed sediments could change, depending on both the stream stage and the aquifer head calculated during the time step. The RIV Package requires that a conductance term $\left(\frac{K W L}{b}\right)$ be specified for the streambed sediments. In the SFR Package, the conductance term is calculated from hydraulic conductivity, stream width, stream length, and streambed sediment thickness, which is specified in the data input.

When the head in the aquifer is below the bottom of the streambed sediments, leakage from the stream to the underlying aquifer is no longer dependent on aquifer head; in this case, flow across the streambed sediments is computed as

$$
Q S F R_{n b}=S_{S F R, n b}^{*} \frac{K_{n b} L_{n b} W_{n b}}{b_{n b}}\left(H S F R_{n b}-S B O T_{n b}\right),
$$

where $S B O T_{n b}$ is the bottom elevation of the streambed sediments (L). Equation 7-2 assumes that the head at the bottom of the streambed sediments is equal to the elevation of the bottom of the streambed sediments. Implied in this method of calculating leakage to the aquifer is that the interval between the bottom of the streambed sediments and the water table has a unit downward gradient, that flow across the streambed sediments is translated directly to the water table without delay, and that the leakage rate does not exceed the saturated hydraulic conductivity of the deposits or rocks that occupy the interval between the bottom of the streambed sediments and water table. Such assumptions generally are reasonable when the unsaturated interval is thin or when long periods of steady (constant) flow are modeled, and the hydraulic gradient across the streambed sediments does not greatly exceed one. In transient simulations, the leakage rate across the streambed sediments can change depending, on the head of the stream. The hydraulic gradient across the streambed is reported for each reach and it is left to the user to decide if the gradients are reasonable. Gradients that greatly exceed one may result in unreasonably large leakage rates. If the leakage rates are unreasonable, streambed and aquifer properties used for that reach in the model might need to be examined.

The cellids of each stream reach are specified in the model input. If the cell corresponding to the stream reach is inactive, simulated stream leakage is applied to the uppermost active cell vertically underlying the specified location. If all cells beneath the stream reach are inactive or if the cell corresponding to the stream reach is set as a constant head, the SFR Package will continue to route flow through the stream reach, but leakage (either into the aquifer or out of the aquifer) across the streambed will not occur in the reach.

\section{Reach Water Budgets}

A stream water budget for each stream reach, as well as the leakage rate between a stream reach and corresponding model cell, is computed each iteration of a time step and at the end of each time step. These calculations are made independent of the groundwater model budget. The water budget of the first reach is computed by determining all the inflows and outflows. Any flow out of the first reach is then routed to the next downstream reach, where a new water budget is determined for that reach; flow out of that reach is then routed to the next downstream reach, and the process is continued to the last reach. Flow out of reaches without downstream connections and the last reach, if it is not connected to any other reaches, leaves the surface-water system represented with the SFR Package, unless the MVR Package is used to route downstream outflow from these reaches to other packages. 


\section{7-4 Documentation for the MODFLOW 6 Groundwater Flow Model}

A. Connected reaches in a model cell

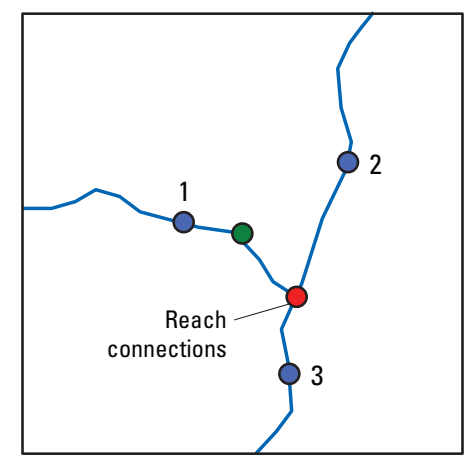

B. Parallel reaches in a model cell

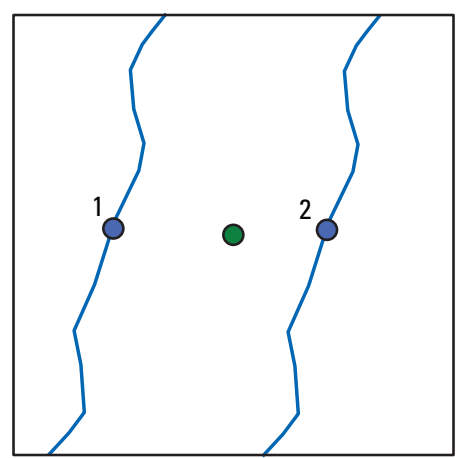

EXPLANATION

3 Stream reach number

- Stream

O Reach connection

Midpoint of stream reach

Cell center
C. One reach per model cell

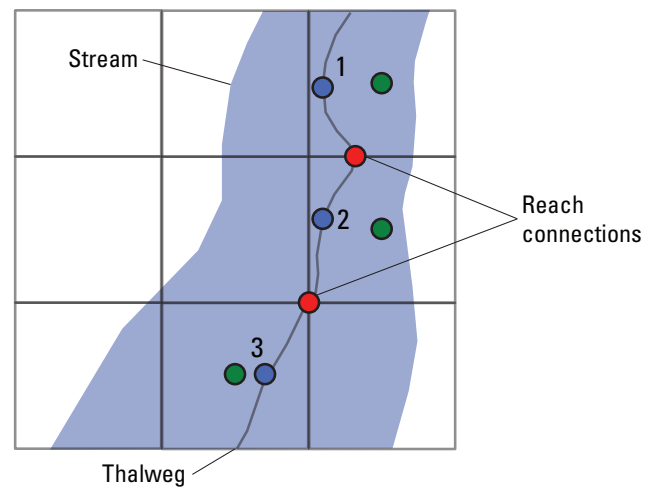

Figure 7-2. Diagram showing several different stream reach configurations. $A$, multiple connected reaches in a model cell, $B$, two parallel reaches in a model cell, and $C$, only one reach connected to a single model cell even if stream is wider than the cell. Figure modified from Prudic and others (2004).

The water budget of each stream reach is used to determine the quantity of streamflow available to leak into the aquifer during each time step. For example, if flow into the reach from all sources is zero (the stream is dry), then no leakage is allowed from that reach into the underlying aquifer. However, if the head in the underlying aquifer is greater than the streambed, then groundwater flow into a reach of zero flow is computed, and the stream then begins to flow in that reach during the time step. Additionally, the outflow from a reach can be less than the leakage to groundwater in the next downstream reach and, if streamflow into the downstream reach from all sources (outflow from upstream reach, overland runoff, and precipitation) is less than the computed quantity of leakage through the streambed, then the leakage across the streambed is limited to the available streamflow in the reach and the stream then goes dry (there is no outflow from that reach).

The program allows several sources of inflow to a stream reach. These sources include a specified inflow $\left(Q S R I_{n b}\right)$, the sum of tributary flow from upstream reaches $\left(Q T R B_{n b}\right)$, direct overland runoff to a reach $\left(Q R O_{n b}\right)$, inflow from the Mover Package $\left(Q M V R_{n b}\right)$, precipitation that falls directly on a reach $\left(Q P P T_{n b}\right)$, and groundwater leakage to a reach calculated by the model $\left(Q S F R I_{n b}\right)$, as shown in the following equation:

$$
Q I N_{n b}=Q S R I_{n b}+Q T R B_{n b}+Q R O_{n b}+Q M V R_{n b}+Q P P T_{n b}-Q S F R I_{n b}
$$

All terms are in units of volume per time. The term $Q S F R I_{n b}$ is subtracted from the other terms because groundwater flow to a stream reach (a source of water to a stream reach) is a negative value (discharge from groundwater is negative in MODFLOW 6). The program also allows for several losses from a stream reach. These losses include streamflow out of a reach $\left(Q S R O_{n b}\right)$, specified diversions from another reach $\left(Q D I V_{n b}\right)$, evaporation from a reach $\left(Q E T_{n b}\right)$, and leakage to the underlying aquifer $\left(Q S F R O_{n b}\right)$, as shown in the following equation:

$$
Q O U T_{n b}=Q S R O_{n b}+Q D I V_{n b}+Q E T_{n b}+Q S F R O_{n b}
$$




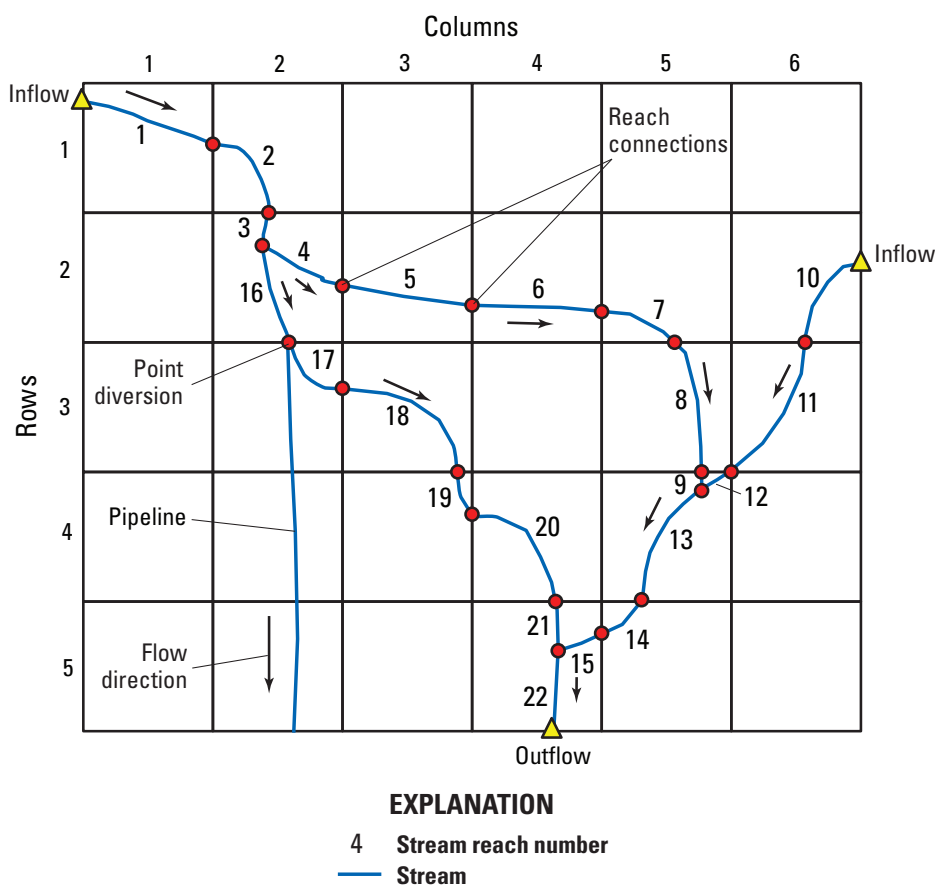

Figure 7-3. Diagram showing an example of a reach-numbering scheme in relation to direction of flow for a regular MODFLOW grid. Figure modified from Prudic and others (2004).

The term $Q S F R O_{n b}$ is added to the equation only when a stream reach loses flow to the aquifer (recharge to the aquifer is a positive value). For each reach, the sum of flows (in units of volume per time) into the reach is equal to the sum of flows out of the reach $\left(Q O U T_{n b}\right)$, as shown below.

$$
Q I N_{n b}=Q O U T_{n b}
$$

Specified inflows and outflows can be defined for each reach. This specified flow can represent streamflow entering the first reach in the modeled area or it can be used to represent localized discharge into a stream (for example, from a pipeline) within the modeled area. For reaches within the modeled area, the specified flow can be negative, in which case the flow would be subtracted from any incoming upstream reach. See fig. 7-3 for an example of a point diversion into a pipeline. Tributary inflows to a reach are computed by the program by summing all outflows from upstream reaches that are tributary to a particular downstream reach. Diversions can be simulated from any reach. The quantity of flow to a diversion (such as an unlined canal or ditch) can be specified to be (1) a fixed rate not to exceed the available flow out of the reach (UPTO), (2) a fixed rate once the available flow out of the reach exceeds the specified value (THRESHOLD), (3) a percentage of the available flow out of the reach (FACTOR), or (4) the difference between available flow out of the reach and the specified values once the specified value is exceeded (EXCESS).

Uniform overland flow (volume per time) can be specified for any reach. Also, uniform precipitation and evaporation rates (units of length per time) can be specified for each reach. Volumetric precipitation and evaporation rates for each reach are then computed by multiplying each rate by the reach length and width.

\section{Computing Reach Depth}

Stream depth is used in the program to compute the stage in each reach by adding stream depth to the top of the streambed for each reach. Stream depth is computed or specified at the midpoint of each active or simple routing reach, respectively. A continuity-based flow at the midpoint $\left(Q_{m d p t_{n b}}\right)$ is computed prior to com- 


\section{7-6 Documentation for the MODFLOW 6 Groundwater Flow Model}

$Q R O+Q P P T=Q E T+Q S F R$

$Q R O+Q P P T>Q E T+Q S F R$

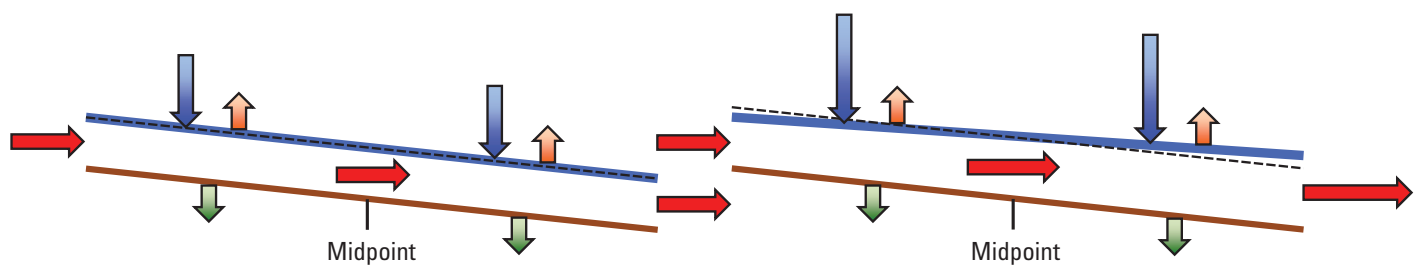

EXPLANATION

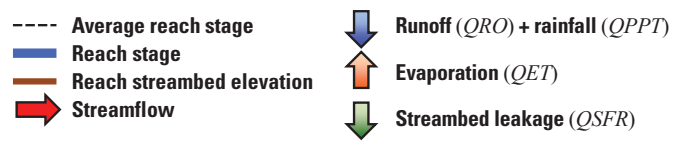

$Q R O+Q P P T<Q E T+Q S F R$

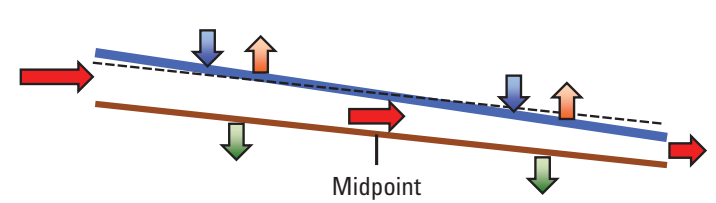

Figure 7-4. Diagram showing relation between the streamflow at the midpoint $Q_{m d p t_{n b}}$ and end of a reach and the sum of $Q R O_{n b}, Q P P T_{n b}, Q E T_{n b}$, and $Q S F R_{n b}$. Note that the average reach stage and the reach stage are only equal when the sum of $Q R O_{n b}, Q P P T_{n b}, Q E T_{n b}$, and $Q S F R_{n b}$ are zero.

puting stream depth (active reaches) or evaluating the specified stream depth and calculated streambed leakage relative to net inflows to the reach (simple routing option reaches). Flow at the midpoint based on continuity is computed as

$$
Q_{m d p t_{n b}}=Q S R I_{n b}+Q T R B_{n b}+Q M V R_{n b}+0.5\left(Q R O_{n b}+Q P P T_{n b}-Q E T_{n b}-Q S F R_{n b}\right)
$$

where $Q S F R_{n b}$ is the leakage through the streambed and includes leakage from the stream reach to the aquifer $\left(Q S F R O_{n b}\right)$ and from the aquifer to the stream reach $\left(Q S F R I_{n b}\right)$. The leakage term is positive when leakage is from the stream reach to the aquifer and negative when leakage is from the aquifer to the stream reach, hence, it is subtracted from the other terms. Because flow at the midpoint of a reach is partly dependent on streambed leakage $\left(Q S F R_{n b}\right)$, which is dependent on stream depth and, therefore, on flow, equation 7-6 is nonlinear, and is solved iteratively using a mixed bisection-Newton-Raphson method until the residual of the reach continuity equation is within a specified tolerance.

A consequence of equation 7-6 is $Q_{m d p t_{n b}}$ will not be equal to the downstream discharge from a reach if the sum of $Q R O_{n b}$ and $Q P P T_{n b}$ are not equal to the sum of $Q E T_{n b}$ and $Q S F R_{n b}$. For example, if the sum of $Q R O_{n b}$ and $Q P P T_{n b}$ is greater than the sum of $Q E T_{n b}$ and $Q S F R_{n b}$, inflows to the reach will exceed outflows and the flow at the downstream end of the reach will be greater than $Q_{m d p t_{n b}}$ (fig. 7-4). Conversely, if the sum of $Q R O_{n b}$ and $Q P P T_{n b}$ is less than the sum of $Q E T_{n b}$, and $Q S F R_{n b}$, outflows to the reach will exceed inflows and the flow at the downstream end of the reach will be less than $Q_{m d p t_{n b}}$ (fig. 7-4).

\section{Active Reaches}

The SFR Package in MODFLOW 6 assumes each active reach is rectangular and uses Manning's equation to determine stream depth as a function of streamflow, unless the simple routing option is used for a reach. This differs from the SFR Package in MODFLOW-2005, which included four options for calculating stream depth. The relation between streamflow and stream depth is

$$
Q_{n b}=\frac{C_{u}}{n_{n b}} A_{S_{n b}} R_{n b}^{2 / 3} S_{0_{n b}}^{1 / 2},
$$


where $Q_{n b}$ is the stream discharge at the midpoint of reach $n b\left(\mathrm{~L}^{3} \mathrm{~T}^{-1}\right) ; C_{u}$ is a units constant, which is 1.0 for units of $\mathrm{m}^{3} \mathrm{sec}^{-1}$ or 1.486 for units of $\mathrm{ft}^{3} \mathrm{sec}^{-1} ; n_{n b}$ is Manning's roughness coefficient $\left(\mathrm{TL}^{-1 / 3}\right) ; A_{S_{n b}}$ is the cross-sectional area of the stream $\left(\mathrm{L}^{2}\right) ; R_{n b}$ is hydraulic radius of the stream (L); and $S_{0_{n b}}$ is slope of the stream channel (unitless).

Assuming a wide rectangular stream channel in which the stream width is much greater than the stream depth, equation 7-7 can be simplified to

$$
Q_{n b}=\frac{C_{u}}{n_{n b}} W_{n b} D_{n b}^{5 / 3} S_{0_{n b}^{1 / 2}}
$$

where $D_{n b}$ is the stream depth at the midpoint of reach $n b(\mathrm{~L})$. Solving for depth yields

$$
D_{n b}=\left[\frac{Q_{n b} n_{n b}}{C_{u} W_{n b} S_{0_{n b}}^{1 / 2}}\right]^{3 / 5} .
$$

Because leakage through the streambed is a function of depth, a mixed bisection-Newton-Raphson method is used to reduce the difference between the streamflow at the midpoint of a reach calculated using equations 7-6 and 7-8 and computed stream depth. Depth $(D)$ is solved by iteratively computing flow for an estimated depth until the difference between computed flow at the midpoint of the reach $\left(Q_{n b}\right)$ and streamflow at the midpoint of the reach $\left(Q_{m d p t_{n b}}\right)$ are acceptably small. The generalized form of the Newton-Raphson equation used to determine stream depth is

$$
D_{n b}^{k}=D_{n b}^{k-1}-f\left(D_{n b}^{k-1}\right) \frac{\left(D_{n b}^{k-1}-\left(D_{n b}^{k-1}+\epsilon\right)\right)}{f\left(D_{n b}^{k-1}\right)-f\left(D_{n b}^{k-1}+\epsilon\right)},
$$

where $k$ is the iteration number and $f\left(D_{n b}^{k-1}\right)$ is $Q_{n b}^{k-1}-Q_{m d p t_{n b}}^{k-1}$ for depth $D_{n b}^{k-1}$.

The bisection-Newton-Raphson procedure starts with two initial estimates of depth $\left(D_{n b}^{k=0}\right.$ and $\left.D_{n b}^{k=1}\right)$ as shown in figure 7-5. The two initial depth estimates are a depth of zero $\left(D_{n b}^{k=0}\right)$ and an arbitrary depth based on the current reach stage and the groundwater head relative to the reach bottom $\left(D_{n b}^{k=1}\right)$, respectively. Initially, the bisection method is used to bracket the interval around the converged depth $\left(D_{n b}\right)$. The bisection method simply halves the difference between the two initial depths $\left(D_{n b}^{k=2}\right.$ in fig. 7-5) and the solution is bracketed to be in the interval between $D_{n b}^{k=2}$ and $D_{n b}^{k=1}$. In the next iteration, the Newton-Raphson method is used to calculate the finite-difference derivative at depth $D_{n b}^{k=2}$. However, as the finite-difference derivative and equation 7-10 result in a depth that exceeds $D_{n b}^{k=1}$, bisection is used to determine $\left(D_{n b}^{k=3}\right)$ and the solution is bracketed to be in the interval between $D_{n b}^{k=3}$ and $D_{n b}^{k=1}$. The finite-difference derivative calculated at $\left(D_{n b}^{k=3}\right)$ results in $D_{n b}^{k=4}$, which is within the current interval $\left(D_{n b}^{k=3}\right.$ and $\left.D_{n b}^{k=1}\right)$ and has a positive $D_{n b}^{k=4}$. The finite-difference derivative calculated at $\left(D_{n b}^{k=5}\right)$ results in $D_{n b}^{k=5}$. The process is repeated until the depth $\left(D_{n b}\right)$ produces a computed flow $\left(Q_{n b}\right)$ that is within an acceptable error of streamflow at the midpoint of each reach $\left(Q_{m d p t_{n b}}\right)$.

\section{Simple Routing Reaches}

For reaches that use the simple routing option, stream stage is user specified or is set to the top of the reach and remains constant during a time step, as long as the calculated streambed leakage to the aquifer is less than or equal to the net inflow to the reach. Like active reaches, simple routing reaches are assumed to be rectangular. If the calculated streambed leakage to the aquifer is greater than the net inflow to the reach, the reach 


\section{7-8 Documentation for the MODFLOW 6 Groundwater Flow Model}

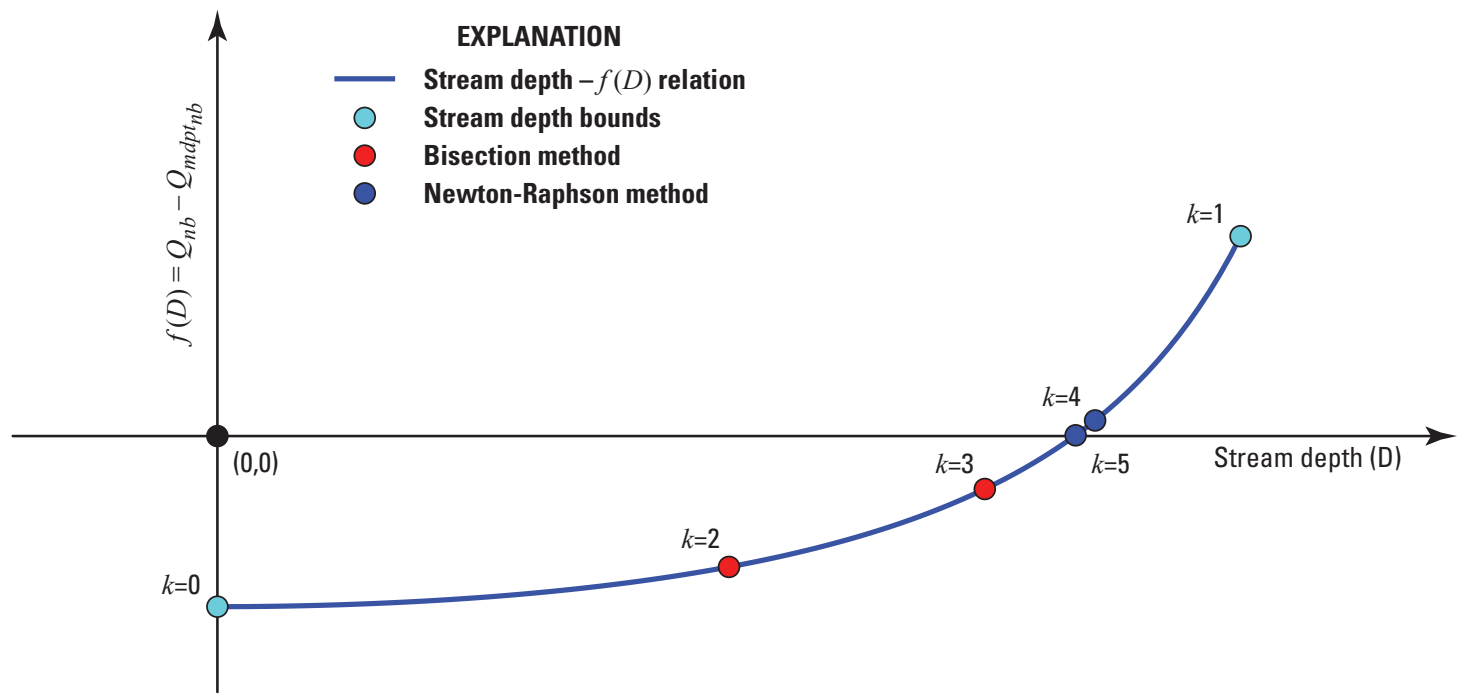

Figure 7-5. Graph showing simulated stream depth with consecutive iterations of the mixed bisection-Newton-Raphson method. This method is used when the Manning's equation is used to simulate stream flow. Figure modified from Prudic and others (2004).

stage is set to the top of the reach (stream depth $=0$ ) and streambed leakage to the aquifer is set equal to the net inflow to the reach. For reaches that use the simple routing option, the flow at the downstream end of the reach is set equal to the sum of the net inflow to the reach and streambed leakage. Furthermore, the flow at the downstream end of the reach will exceed the net inflow to the reach if the head in the aquifer exceeds the stream stage in reaches that use the simple routing option.

\section{Diverting Streamflow}

Water entering a diversion is subtracted from the source reach; any remaining flow (after all diverted water from a reach is removed) at the end of the reach is added to the next downstream reach of the source reach. If the source reach has more than one connected downstream reach, then apportioning of the remaining water to the downstream reaches is based on user-specified fractions. Water from a diversion does not interact with groundwater through a leakage term. For diversions, it is assumed the water is placed in a nonleaking pipe or a lined canal. An example of such a point diversion is shown in figure 7-3.

If a diversionary flow is large enough to warrant representation in the model, but is discharged into a pipeline, lined canal, or other structure or system that does not interact with the aquifer and the flow might exceed the available streamflow, then there is an alternative means to represent it. A single diversion reach can be assigned to the same model cell as the upstream reach from which the flow is to be diverted. Groundwater interaction with this single diversion reach can be eliminated by assigning a hydraulic conductivity of zero and outflow to a nondefined reach (for example, reach 0 ). Diversion of water into the reach would then be subject to the constraints associated with the type of diversion specified, as described in the following paragraph.

Water can be diverted from stream reaches using several different diversion types. The simplest diversion type is a specified flow (negative discharge) that is removed from the channel at the specified reach. The SFR Package has four additional diversion types for calculating the diversion flow rate. The first calculated diversion type (UPTO diversion) assumes that all available flow at the end of the reach up to the user-specified rate will be diverted from the reach. Thus, if flow in the stream is less than the specified diversion, all available flow in the stream will be diverted and none will remain in the channel. This option is common to many diversions in the Western United States. The second calculated diversion type (THRESHOLD diversion) allows for the specified diversion only if flow in the stream is greater than that of the diversion; if flow is less than 
the specified diversion, no flow is diverted and all flow remains in the channel. This option is the same as that specified in the STR1 Package (Prudic, 1989). The third calculated diversion type (FACTOR type) diverts a specified fraction of the available flow, and the fourth calculated diversion type (EXCESS type) diverts all flow in excess of a specified rate. The fourth calculated diversion type typically is used for flood control in which all excess flow is diverted away from the main channel during peak discharge. The last two calculated diversion types (FACTOR and EXCESS types) are from Danskin and Hanson (2002).

Diversions from stream reaches require knowledge as to when and where such diversions occur. The overall structure of the SFR Package has been designed to allow for the hierarchical ranking (or priority) of a diversion relative to other diversions in the overall stream network. This is analogous to water rights that have priorities in time under the regulations of many Western States.

\section{Assumptions and Limitations}

A major limitation of the SFR package is that it can only represent stream reaches as a wide rectangular cross section with a single Manning's roughness coefficient (for active reaches). Previous versions of the SFR Package (for example, Niswonger and Prudic, 2005) supported representing stream cross sections with an eight-point cross section with different Manning's roughness coefficients for the channel and the left and right banks. Other surface-water simulation codes typically include additional reach geometry and Manning's roughness coefficient options (for example, Barkau, 1996 and Hughes and others, 2012). The assumption that reach geometry can be represented as a single wide rectangular cross section might result in notable errors if the actual reach hydraulic radius and connection area with the underlying aquifer increased with increasing depth and the reach spent a significant amount of time out of bank, subjected to increased Manning's roughness coefficients. The differences in Manning's roughness coefficients could be partially compensated for by using difference Manning's roughness coefficients during the simulation. For most long-term simulations with MODFLOW 6, it is not expected that the wide rectangular cross-section assumption will have a notable impact on simulated streamflow and stream leakage.

A second limitation of the SFR Package is that the method used to compute stream depth at the midpoint of each stream reach may not always converge to a stable solution because of the dependency between computed stream stage and leakage that is solved using mixed bisection-Newton-Raphson method. In the SFR Package, head in the stream is computed at the midpoint of the reach within a model cell and includes half the precipitation, evaporation, overland runoff, and leakage across the streambed that occurs within the reach. Oscillations are more common when stream stage is nearly the same as that in the aquifer, and flow across the streambed oscillates between adding water to the aquifer and discharging water from the aquifer. Instability is greatest for steady-state simulations because the groundwater storage term is zero and for simulations in which the streambed conductance term is high relative to the aquifer conductivity, especially when the head difference between the stream and aquifer is small. In some cases, oscillation can be eliminated by increasing the stream depth change tolerance and decreasing the streambed hydraulic conductivity.

Another limitation that needs to be considered when using the SFR Package is that streamflows are routed between stream reaches based only on continuity. This means that during each model time step, volumetric inflow and outflow rates are equal for each stream reach, that no water is added to or removed from storage in the surface channels, and that any water that enters the aquifer by leakage in a losing stream reach is not routed to the next downstream reach of the stream. The SFR Package was designed to model long-term changes (months to hundreds of years) in groundwater flow using averaged flows in streams. The continuity approach taken in the SFR Package has general applicability because groundwater flow velocities are usually much less than flow velocities in open channels. The SFR Package is not recommended for modeling the transient exchange of water between stream reaches and shallow groundwater when the objective is to examine short-term (minutes to days) effects caused by rapidly changing streamflows. 


\section{7-10 Documentation for the MODFLOW 6 Groundwater Flow Model}

The assumption that leakage through the streambed is transmitted to the water table instantaneously when the aquifer head is below the bottom elevation of the streambed generally limits the applicability of the SFR Package to the simulation of transient leakage through thin unsaturated or nearly saturated zones beneath streams, unless the UZF Package is used under the reach. This assumption may not be reasonable for some intermittent or ephemeral channels in which the water table is tens to hundreds of feet or meters below the streambed. Also, when the water table is below the bottom of the streambed, flow across the streambed is dependent on the head difference across the streambed (the difference between the stage in the stream and the bottom of the streambed), and the rate of leakage is not dependent on the hydraulic properties of the materials beneath the streambed.

For complex models with many stream reaches, differences in the timing and response among individual stream reaches with their corresponding model cells caused by variations in streamflow and groundwater pumping may require more time steps to adequately simulate the exchange of water for all model cells with a stream reach. Because of the difficulty in knowing how and when changes in the head difference between a stream reach and corresponding model cell will result in large changes in leakage rates, the user is cautioned to check stream leakage for all stream reaches to make certain that sufficient time steps have been used to adequately simulate changes in all reaches.

\section{Incorporation of Leakage Into the Groundwater Flow Equation}

After the solution of the continuity equation for each reach is calculated, leakage is incorporated into the CVFD flow equation (eq. 2-24) based on whether the standard or Newton-Raphson formulation is being used. The details of how SFR Package leakage is incorporated into the CVFD flow equation is described below.

\section{Standard Formulation}

At the start of each iteration, terms representing stream seepage are added to the flow equation for each cell containing a stream reach. The choice of which streambed seepage equation to use is made by comparing the most recent value of head at the cell to the bottom of the streambed and the sum of available sources and sinks for a reach. The sum of available sources and sinks for a reach is calculated as

$$
Q S R C_{n b}=Q S R I_{n b}+Q T R B_{n b}+Q R O_{n b}+M V R_{n b}+Q P P T_{n b}-Q E T_{n b} .
$$

Because this process is done at the start of each iteration, the most current value of head $\left(h_{n}\right)$ is the value from the previous iteration. Thus, the check for which river seepage equation to use lags behind the seepage calculations by one iteration. In cases where the head in cell $n$ is at or below the bottom of the streambed, flow from the SFR package is not head dependent and is calculated as

$$
\begin{array}{lr}
Q S F R_{n b}=Q S R C_{n b} & \text { if } Q S F R_{n b} \geq Q S R C_{n b} \\
Q S F R_{n b}=S_{S F R, n b}^{*} \operatorname{CSFR}_{n b}\left(H S F R_{n b}-S B O T_{n b}\right) & \text { if } Q S F R_{n b}<Q S R C_{n b}
\end{array}
$$

where $C S F R_{n b}$ is the streambed conductance $\left(\mathrm{L}^{2} \mathrm{~T}^{-1}\right)$. Because SFR leakage is not head dependent in this case, $Q S F R_{n b}$ is subtracted from the right-hand side of equation 2-24.

For cases where the GWF head in cell $n$ is above the streambed bottom and the SFR leakage is less than the sum of available sources and sinks for a reach, then the term $C S F R_{n b}$ is added to the diagonal of the coefficient matrix in equation 2-24 for cell $n$. The term $C S F R_{n b} \times H S F R_{n b}$ is subtracted from the right-hand side of equation 2-24. Finally, for cases where the GWF head in cell $n$ is below the streambed and the SFR leakage 
is greater than or equal to the sum of available sources and sinks for a reach, $Q S R C_{n b}$ is subtracted from the right-hand side of equation 2-24.

\section{Newton-Raphson Formulation}

SFR Package reaches can act as either head-dependent boundaries or as specified flow boundaries, depending on the hydrologic conditions. Reaches are specified flow boundaries when $h_{n}<S B O T_{n b}$ or when $Q S F R_{n b}>Q S R C_{n b}$. For specified flow boundaries, the derivative is zero. For head-dependent conditions, finite differences are used to calculate the derivatives. The derivatives are calculated as

$$
\frac{\partial Q S F R_{n b}}{\partial h_{n}}=\frac{Q S F R_{n b}^{+}-Q S F R_{n b}}{\epsilon_{h}},
$$

where $Q_{S F R}^{+}$is the streamflow boundary leakage to the aquifer at $h+\epsilon_{h}$ and $\epsilon_{h}$ is a small perturbation value. For cases where a streamflow boundary is head dependent $\left(h_{n}+\epsilon_{h}>S B O T_{n b}\right)$, the resulting NewtonRaphson linear equation is

$$
\frac{\partial Q S F R_{n b}}{\partial h_{n}} h_{n}^{k}=-Q S F R_{n b}+\frac{\partial Q S F R_{n b}}{\partial h_{n}} h_{n}^{k-1}
$$

For head-dependent cases, the terms added to equation 2-24 as part of the standard formulation are manipulated to formulate the Newton-Raphson equations. The additional operations include: (1) subtracting $C S F R_{n b}$ from the diagonal of the coefficient matrix in equation 2-24, (2) subtracting the term $C S F R_{n b} \times H S F R_{n b}$ from the right-hand side of equation 2-24 to complete the residual $\left(Q S F R_{n b}\right)$, (3) addition of $\frac{\partial Q S F R_{n b}^{m-1}}{\partial h_{n}}$ to the diagonal of the coefficient matrix in equation 2-24, and (4) addition of $\frac{\partial Q S F R_{n b}^{m-1}}{\partial h_{n}} \times h_{n}^{m-1}$ to the righthand side of equation 2-24. Equation 2-24 is not modified for the Newton-Raphson case if the leakage does not depend on the simulated aquifer head. 


\section{7-12 Documentation for the MODFLOW 6 Groundwater Flow Model}

\section{Lake Package}

Quantifying the hydraulic relation between a lake and the adjacent aquifer requires a method for estimating the amount of water exchanged between the two domains by seepage through the materials that separate them. The Lake (LAK) Package applies Darcy's Law and the difference between the head in the aquifer with the stage of the lake to calculate seepage. The LAK Package uses the continuity equation based on the components of the lake water budget, such as rainfall recharge, evaporation, overland runoff, surface-water inflows and outflows, lake storage changes, and direct withdrawals from the lake or augmentation of the lake volume by anthropogenic means. Newton's method is used to solve for lake stage based on the lake continuity equation. If the lake dries appreciably, then the surface area may decrease, substantially affecting other processes controlling the lake water budget; consequently, lake computations account for changes in the surface area of the lake and the dependence of water-budget components on lake stage.

\section{Lake-Aquifer Connection}

The direction and magnitude of seepage between a lake and the adjacent aquifer system depends on the relation between the lake stage and the hydraulic head in the groundwater system, both of which can vary substantially in time and space. Seepage from a lake into the aquifer that surrounds it, where the lake acts as a source of recharge to the aquifer, occurs when and where the lake stage is higher than the altitude of the water table in the adjacent part of the aquifer. Typical situations in which substantial recharge to the aquifer occurs are those where a lake receives surface inflows in excess of outflows, perhaps from a stream discharging into the lake, or where the water level in the aquifer is drawn down by pumpage from wells. Seepage from the aquifer into a lake usually occurs where the water-table altitude is normally higher than that of the lake. Such cases are found in karst regions where lakes commonly have no substantial inflows or outflows. In these environments, because less water per unit volume is stored in the aquifer than in the lake, periods of rainfall cause the water table to rise higher than the lake stage, thus increasing the rate of seepage from the aquifer into the lake. In this manner, the lake can act as a hydraulic sink for the groundwater system. In still other hydrologic environments, a lake can represent a mixed or "flow-through" condition where, in some areas of the lakebed, seepage is into the lake, and in other areas, seepage is out of the lake.

Conceptually, the method of computing flow between lakes and aquifers is the same as that used for the River Package. Flow between lakes and aquifers in the GWF Model is computed using Darcy's Law and assuming uniform flow between a lake and the connected aquifer cell. This flow, $Q L A K_{n b}$, is computed as

$$
Q L A K_{n b}=\frac{K_{n b} A_{n b}}{L_{n b, n}}\left(H L A K_{l}-h_{n}\right)
$$

where $K_{n b}$ is the effective hydraulic conductivity of the sediments underlying the lake and aquifer materials $\left(\mathrm{LT}^{-1}\right) ; A_{n b}$ is the wetted area of the lake, in the cell, perpendicular to flow $\left(\mathrm{L}^{2}\right) ; L_{n b, n}$ is the distance from the lake bottom $\left(L B O T_{n b}\right)$ to the center of the connected GWF Model cell in the appropriate coordinate direction (fig. 7-6, the distance between the edge of the aquifer grid cell that is the interface with the lake bottom and the aquifer grid cell center) (L); $H L A K_{l}$ is the simulated stage in the lake (L); and $h_{n}$ is the head at the node underlying the lake $(\mathrm{L})$. The area of the lake-aquifer connection perpendicular to flow is calculated as

$$
\begin{array}{lr}
A_{n b}=S_{F_{n b}}^{*} w_{n b}\left(T O P_{n b}-B O T_{n b}\right), & \text { if connection is horizontal } \\
A_{n b}=S_{F_{n b}}^{*} A_{n}, & \text { if connection is vertical } \\
A_{n b}=A_{l}, & \text { if connection is embedded. }
\end{array}
$$




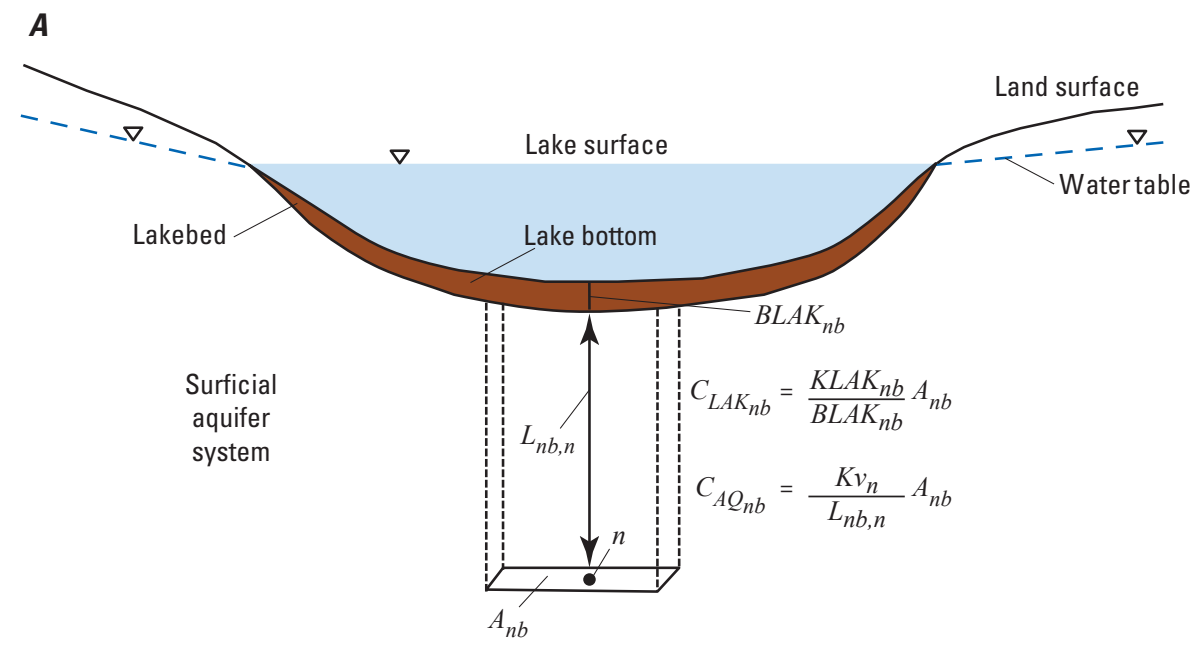

B

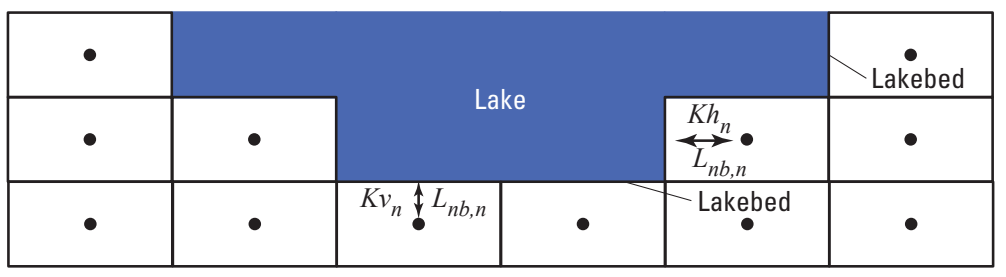

EXPLANATION

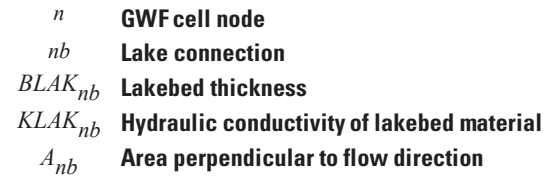

$\begin{array}{cl}L_{n b, n} & \text { Distance between lakebed and GWF cell node } \\ K h_{n} & \text { Aquifer horizontal hydraulic conductivity } \\ K v_{n} & \text { Aquifer vertical hydraulic conductivity } \\ C L A K_{n b} & \text { Lakebed conductance } \\ C_{A Q_{n b}} & \text { Aquifer conductance }\end{array}$

Figure 7-6. Diagram showing the concepts and length parameters used to calculate seepage between a lake and the aquifer. Figure modified from Merritt and Konikow (2000).

where $S_{F_{n b}}^{*}$ is the lake saturation (unitless), $w_{n b}$ is the horizontal width of the lake-aquifer connection cell (L), $T O P_{n b}$ is the top of the lake-aquifer connection cell (L), $B O T_{n b}$ is the bottom of the lake-aquifer connection cell (L), $A_{n}$ is the horizontal area of cell $n\left(\mathrm{~L}^{2}\right)$, and $A_{l}$ is the surface area of lake $l\left(\mathrm{~L}^{2}\right)$.

For horizontal connections, $S_{F_{n b}}^{*}$ is calculated using equation 4-5 and varies from 0 to 1 , based on (1) the groundwater head in the connected cell, (2) the average of the lake stage or groundwater head, or (3) the maximum of the lake stage or the groundwater head (upstream weighting) (fig. 7-7). Upstream weighting of horizontal connections is only applied when the Newton-Raphson formulation is used to incorporate lake leakage into the CVFD equation. Only the groundwater head was used to calculate the lake-aquifer conductance for horizontal lake connections in previous versions of MODFLOW (Merritt and Konikow, 2000).

For vertical connections, $S_{F_{n b}}^{*}$ is 1 for all lake stages and groundwater levels, unless a $S U R F D E P$ value greater than 0 is specified for the lake-aquifer connection. If a $S U R F D E P$ value greater than 0 is specified, $S_{F_{n b}}^{*}$ varies from 0 , when $H L A K_{l}$ and $h_{n}$ is less than or equal to the lake bottom $\left(T O P_{n}\right)$, to 1 at $H L A K_{l}$ or $h_{n}>=T O P_{n}+S U R F D E P$ (fig. 7-8). Use of SURFDEP to smooth the connection area can enhance convergence for vertical lake connections. 


\section{7-14 Documentation for the MODFLOW 6 Groundwater Flow Model}

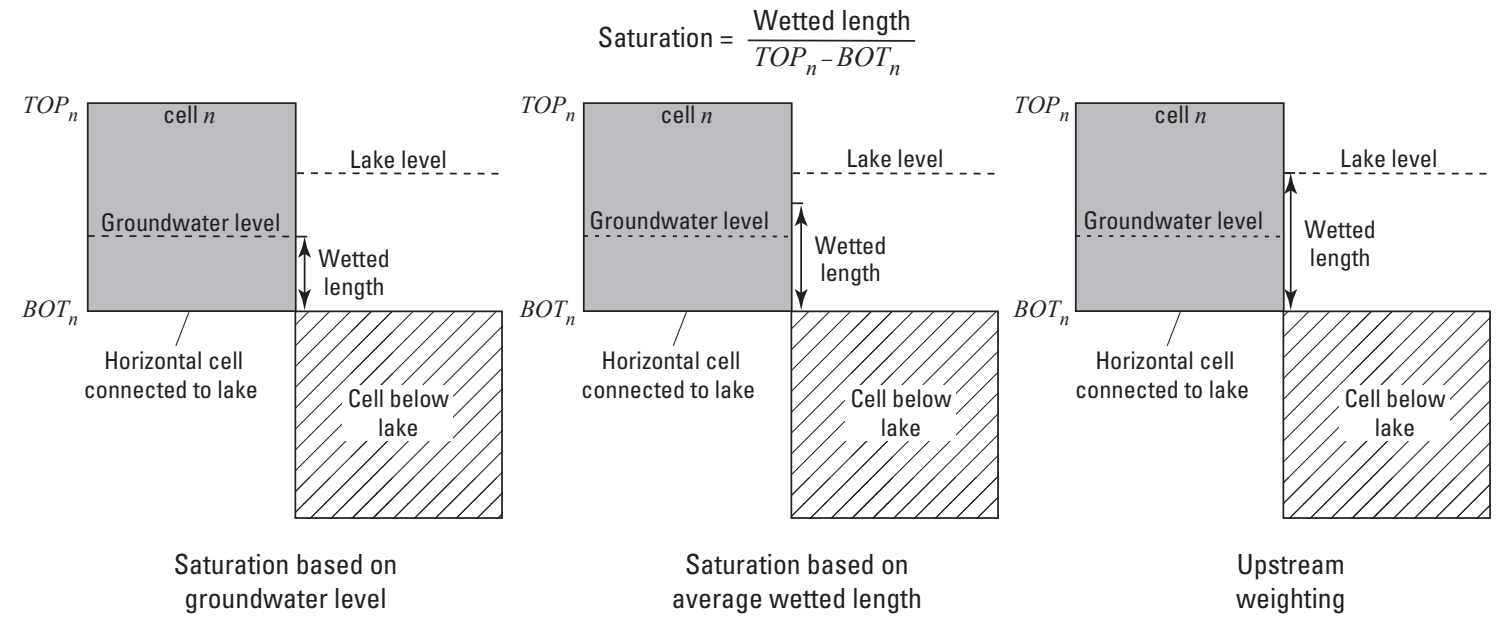

Figure 7-7. Diagram showing the approaches for calculating the saturation for horizontal lake connections to a model cell. Upstream weighting is only applied when the Newton-Raphson formulation is used to incorporate lake leakage into the groundwater flow equation.

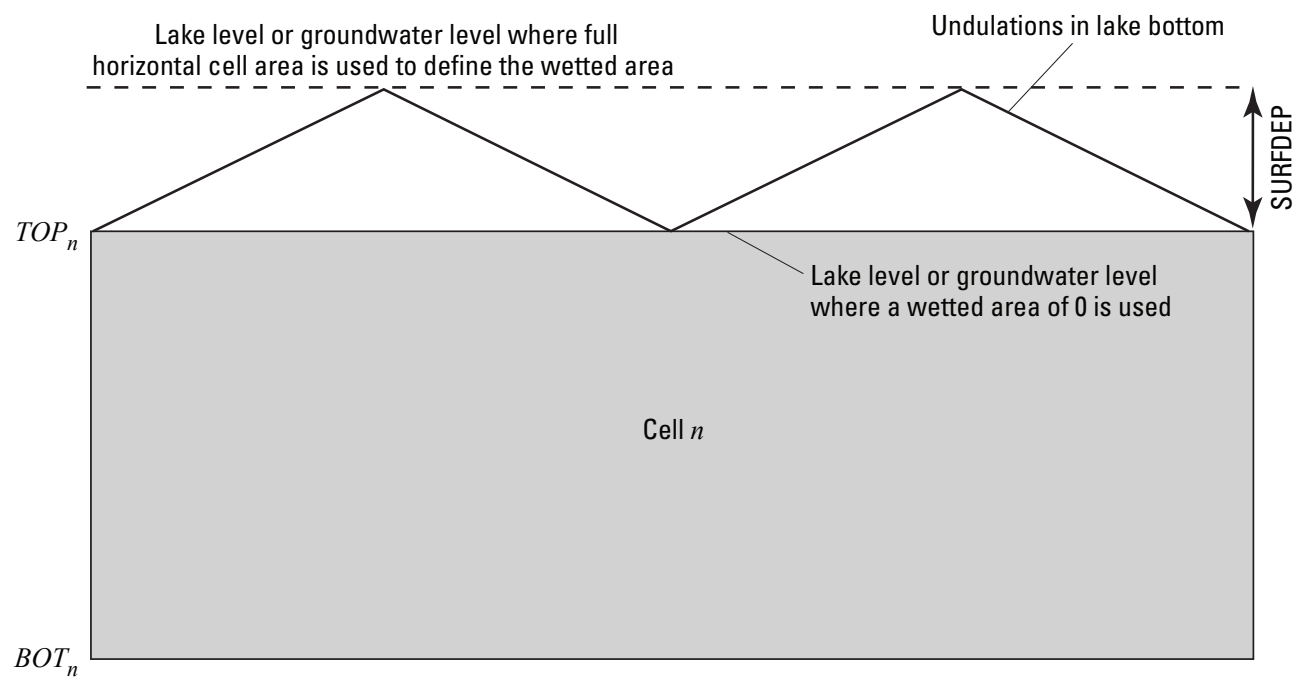

Figure 7-8. Diagram showing the effect of lake surface depth, $S U R F D E P$, at the lake bottom on the wetted area of lakeaquifer connection for vertical connections in relation to the lake level or the water table in a model cell. 
For embedded connections, $A_{l}$ is a function of the simulated lake stage. Furthermore, lakes defined as having embedded connections can only be connected to a single cell and the relation between lake stage and the wetted area is user defined.

In this approach, transient seepage across the lakebed could change, depending on both the lake stage and the aquifer head calculated during the time step. The RIV Package requires that a conductance term $\left(\frac{K A}{L}\right)$ be specified for the river boundary. In the LAK Package, the conductance term is calculated from aquifer hydraulic conductivity, lakebed leakance, and the wetted area of the lakebed for the lake-aquifer connection, which is read in or calculated from the input data. The lakebed leakance, $L L A K_{n b}$, is calculated as

$$
L L A K_{n b}=\frac{K L A K_{n b}}{B L A K_{n b}}
$$

where $K L A K_{n b}$ is the hydraulic conductivity of the lakebed sediments underlying the lake $\left(\mathrm{LT}^{-1}\right)$ and $B L A K_{n b}$ is the thickness of lakebed sediments (L). The conductance for a lake connected to a GWF cell assumes lakebed sediments and the aquifer are connected in series and is calculated as

$$
C L A K_{n b}=\frac{A_{n b}}{\frac{1}{L L A K_{n b}}+\frac{L_{n b, n}}{K_{n}}},
$$

where $C L A K_{n b}$ is the conductance of the connection between the lake and the GWF Model cell $\left(\mathrm{L}^{2} \mathrm{~T}^{-1}\right)$, and $K_{n}$ is the hydraulic conductivity of the aquifer cell underlying the lake connection in the direction of flow (either horizontal, $K_{h}$, or vertical, $K_{v}$ ) $\left(\mathrm{LT}^{-1}\right)$. Embedded lakes use either the horizontal $\left(K_{h}\right)$ or vertical hydraulic conductivity $\left(K_{v}\right)$ based on the user-specified embedded lake connection type when calculating lake conductance.

Either of the terms in the denominator of the right side of equation 7-18 may or may not dominate quantitatively, depending on the properties of the system being simulated. For instance, if the hydraulic conductivity of a 1 -foot (ft) thick lakebed were $0.1 \mathrm{ft}$ per day (ft/d), and the hydraulic conductivity of a 100-ft section of aquifer $\left(L_{n b}=50 \mathrm{ft}\right)$ were $10 \mathrm{ft} / \mathrm{d}$, the two terms in the denominator of equation $7-18$ would be the same. If the lakebed were only $0.1 \mathrm{ft}$ thick and had a hydraulic conductivity of $1 \mathrm{ft} / \mathrm{d}$, the aquifer term would dominate the resulting conductance value. If the aquifer hydraulic conductivity were $1,000 \mathrm{ft} / \mathrm{d}$, the lakebed term would dominate the resulting conductance value.

When the head in the aquifer is below the lake bottom in a connected GWF cell, leakage from the lake to the underlying aquifer is no longer dependent on aquifer head; in this case, flow across the lakebed is estimated by computing the head gradient across the lakebed, assuming that the head at the bottom of the lakebed is equal to the lake bottom. Implied in this method of calculating leakage to the aquifer is that the interval between the lake bottom and the water table has a unit downward gradient, that flow across the lakebed is translated directly to the water table without delay, and that the leakage rate does not exceed the effective saturated hydraulic conductivity of the sediments underlying the lake and aquifer materials. Such assumptions generally are reasonable when the unsaturated interval is thin or when long periods of steady (constant) flow are modeled, and the hydraulic gradient across the lakebed does not greatly exceed one. In transient simulations, the leakage rate across the lakebed can change, depending on the lake head. Gradients that greatly exceed one may result in unreasonably large leakage rates. If the leakage rates are unreasonable, lakebed and aquifer properties used for that lake in the model might need to be examined.

The layer, row, and column or node number of each lake and GWF Model cell connection are specified in the model input. If the cell corresponding to the lake is inactive, simulated lake leakage is applied to the uppermost active cell vertically underlying the specified GWF cell connection location. If all cells beneath the lake and GWF Model cell connection are inactive or if the GWF Model cell connected to the lake is set as a 


\section{7-16 Documentation for the MODFLOW 6 Groundwater Flow Model}

constant head, then leakage (either into the aquifer or out of the aquifer) between the lake and connected GWF cell will not occur.

\section{Lake Outlets}

Volumes of water lost from a lake through surface-water control structures and to downstream streams can be a substantial, if not dominant, part of the lake water budget. At the same time, streams may contribute appreciably to the aquifer water budget by seepage through the streambeds. The mutual interrelation between stream, lake, and aquifer was recognized by Cheng and Anderson (1993). A number of lake outlet types are available and include: (1) specified outflow, (2) Manning's equation outflow, and (3) sharp-crested weir outflow. Lake discharge from a rectangular outlet that uses Manning's equation Chaudhry (2007) is calculated as

$$
\operatorname{QOUTLET}_{n o}=\frac{C_{u}}{n_{n o}} W_{n o} D_{n o}^{\frac{5}{3}}\left(S_{0_{n o}}\right)^{\frac{1}{2}}
$$

where $C_{u}$ is a constant, which is 1.0 for units of $\mathrm{m}^{3} \mathrm{sec}^{-1}$ or 1.486 for units of $\mathrm{ft}^{3} \mathrm{sec}^{-1} ; n_{n o}$ is Manning's roughness coefficient of the outlet $\left(\mathrm{TL}^{-1 / 3}\right) ; W_{n o}$ is the width of the outlet (L); $D_{n o}$ is the water depth above the outlet invert elevation (L); and $S_{0_{n b}}$ is the slope of the outlet channel (unitless). The water depth above the outlet invert elevation is calculated as

$$
D_{n o}=H L A K_{l}-z_{n o},
$$

where $H L A K_{l}$ is the lake stage (L) and $z_{n o}$ is the outlet invert elevation (L). Equation 7-19 is based on the assumptions that the lake outlet is a rectangular channel, that the flow is under channel control (rather than lake outlet control; that is, the outlet channel slope is less than the critical slope), and that the lake outlet channel is hydraulically long so that the normal depth is valid at the lake outlet.

Lake discharge from outlet no that uses the sharp-crested weir equation is calculated as

$$
\operatorname{QOUTLET}_{n o}=\frac{2}{3} C_{d_{n o}} W_{n o} D_{n o}^{\frac{3}{2}}(2 g)^{\frac{1}{2}}
$$

where $C_{d_{n o}}$ is a discharge coefficient (unitless); $W_{n o}$ is the width of the weir outlet (L), $D_{n o}$ is the water depth above the outlet weir invert elevation (L); and $g$ is gravitational acceleration $\left(\mathrm{LT}^{-2}\right)$. All lake weir outlets use a constant $C_{d_{n o}}$ value of 0.61 , which is based on the assumption that the height of water above the weir invert is small relative to the height of the weir invert above the channel bottom (Chaudhry, 2007).

\section{Lake Water Budgets}

A lake water budget for each lake, as well as the leakage rate between a lake and each connected GWF cell, is computed at each iteration of a time step and at the end of each time step. These calculations are made independent of the GWF Model budget. The water budget of each lake is computed by determining all the inflows and outflows to the lake. Any flow out of the lake through an outlet is routed to the specified destination (for example, another lake, an external boundary, or an internal boundary that is picked up by the Mover Package).

The water budget of each lake is used to determine the quantity of lake water available to leak into the aquifer during each time step. For example, if flow into the lake from all sources is zero and there is no water stored in the lake (the lake is dry), then no leakage is allowed from that lake into the underlying aquifer. However, if the head in the underlying aquifer is greater than the lake bottom, then groundwater flow into a lake is 
computed, and water can potentially be lost to leakage in other groundwater cell connections, evaporation, lake withdrawals, and lake outlets during the time step. Additionally, the outflow from a lake can be less than the leakage to groundwater in a downstream lake connected by the MVR Package and, if inflow into the downstream lake from all sources (outflow from upstream lake provided by the MVR Package, overland runoff, and precipitation) is less than the computed quantity of leakage through the lakebed, then the leakage across the lakebed is limited to the available inflow into the lake and there is no outflow from that lake.

The program allows several sources of inflow to a lake (lake $l$ ). These sources include a specified inflow $\left(Q I N F_{l}\right)$, the net outflow from connected lakes $\left(Q O U T L E T_{n o}\right)$, direct overland runoff to a lake $\left(Q R O_{l}\right)$, the sum of inflow from the Mover Package $\left(Q M V R_{l}\right)$, precipitation that falls directly on a lake $\left(Q P P T_{l}\right)$, and the sum of groundwater leakage to a lake calculated by the model $\left(Q L A K I_{n b}\right)$, as shown in the equation below:

$$
Q I N_{l}=Q I N F_{l}+\sum_{\substack{n o=1 \\ n o_{d s} \in l}}^{\text {noutlets }} Q O U T L E T_{n o}+Q R O_{l}+Q M V R_{l}+Q P P T_{l}-\sum_{\substack{n b=1 \\ n b \in l}}^{n c o n n} Q L A K I_{n b}
$$

All terms are in units of volume per time. The term $Q L A K I_{n b}$ is subtracted from the other terms because groundwater flow to a lake (a source of water to a lake) is a negative value (discharge from groundwater is negative in MODFLOW 6 ). The program also allows for several losses from a lake. These losses include outlet flow from a lake $\left(Q O U T L E T_{n o}\right)$, specified withdrawals from the lake $\left(Q W I T H_{l}\right)$, evaporation from a lake $\left(Q E T_{l}\right)$, and leakage to the underlying aquifer $\left(Q L A K O_{n b}\right)$, as shown in the equation below:

$$
Q O U T_{l}=\sum_{\substack{n o=1 \\ n o_{u s} \in l}}^{\text {noutlets }} Q O U T L E T_{n o}+Q W I T H_{l}+Q E T_{l}+\sum_{\substack{n b=1 \\ n b \in l}}^{n c o n n} Q L A K O_{n b}
$$

The term $Q L A K O_{l}$ is added to the equation only when a lake loses flow to the aquifer (recharge to groundwater is a positive value). For each lake, the difference between the sum of flows (in units of volume per time) into the lake and the sum of flows out of the lake is equal to storage changes $\left(Q S T O_{l}\right)$ in the lake, as shown in the following equation:

$$
Q I_{l}-Q O U T_{l}=\frac{V_{l}^{k}-V_{l}^{t_{\text {old }}}}{t-t_{\text {old }}}=Q S T O_{l}
$$

where $V_{l}^{k}$ is the current volume of lake $l\left(\mathrm{~L}^{3}\right)$ and $V_{l}^{t_{\text {old }}}$ is the volume of lake $l$ at the end of the previous time step $\left(\mathrm{L}^{3}\right)$.

Specified inflows and outflows can be defined for each lake. Constant flows into a lake can also be specified for each lake. This specified flow can represent streamflow entering the first lake in the modeled area or it can be used to represent localized discharge into a stream (for example, from a pipeline) within the modeled area. For segments within the modeled area, the specified flow can be negative, in which case the flow would be subtracted from any incoming upstream lake (for example, a point diversion into a pipeline). Tributary inflows to a lake are computed by the program by summing all outflows from upstream lakes that are tributary to a particular downstream lake. Diversions can be simulated from any lake. The quantity of flow to a diversion (such as an unlined canal or ditch) can be specified at a fixed rate or as a percentage of flow out of the lake. 


\section{7-18 Documentation for the MODFLOW 6 Groundwater Flow Model}

Uniform overland flow (volume per time) can be specified for any lake. Also, uniform precipitation and evaporation rates (units of length per time) can be specified for each lake. Volumetric precipitation and evaporation rates for each lake are then computed by multiplying each rate by the lake length and width.

\section{Computing Lake Stage}

Lake stage is calculated using the Newton-Raphson method and numerical derivatives calculated using

$$
\frac{\partial Q_{l}}{\partial h}=\frac{r_{l}\left(h_{l}^{k-1}+\Delta h\right)-r_{l}\left(h_{l}^{k-1}\right)}{\Delta h}
$$

where $r_{l}\left(h^{k-1}+\Delta h\right)^{k-1}$ is the residual at the perturbed lake stage $\left(\mathrm{L}^{3} \mathrm{~T}^{-1}\right)$ and $r_{l}\left(h^{k-1}\right)$ is the residual at the current lake stage $\left(\mathrm{L}^{3} \mathrm{~T}^{-1}\right)$. The residual of the lake water balance is calculated from equation 7-24 as

$$
r_{l}=Q I N_{l}-Q O U T_{l}-Q S T O_{l}
$$

The lake stage for lake $l$ is updated using

$$
h_{l}^{k}=h_{l}^{k-1}-\frac{r_{l}\left(h^{k-1}\right)}{\frac{\partial Q_{l}}{\partial h}} .
$$

The bisection method is used if the initial Newton-Raphson update is not within the defined top and bottom of the lake. In subsequent Newton-Raphson updates, the bisection method is used with the last two lake stage estimates to improve convergence if the Newton-Raphson method "oscillates" or the calculated NewtonRaphson update is outside of the previous two lake stage estimates. The Newton-Raphson method is considered to be "oscillatory" if the sign of lake-aquifer exchanges switches 7 or more times or the NewtonRaphson update switches sign or increases between subsequent iterations 12 or more times. The NewtonRaphson/bisection process is repeated until the change in lake stage is less than a specified value.

\section{Drying and Rewetting of Sections of a Lake}

Because the elevation of the bottom of a lake is spatially variable and rises to an elevation equivalent to the lake stage at the shoreline, lowering the lake stage can potentially dry sections of the lake having higher bottom elevations. Some lakes have irregular bathymetry and also can vary widely in typical stages, a combination that leads to periodic drying of substantial parts of the lake area. Therefore, the Lake Package was coded to represent the drying and rewetting of sections of a lake and the consequent effects on leakage of water with the underlying or adjacent aquifer.

The lake is considered to have horizontal and vertical connections to model grid cells. Therefore, for vertical connections the elevation of the bottom of the lake in a model cell is the user-specified top elevation of the vertically connected cell. If the lake stage is lowered below that elevation, the lake is considered dry in that cell and the calculated surface area of the lake is reduced by the horizontal area of the cell. The discrete nature of the model grid implies that this will be a stepwise representation of a process that occurs more gradationally in nature, unless lake bathymetry data are specified for the lake. Therefore, the effect of gridding on the computation of lake stage could result in a discontinuity in lake stage. This effect can be mitigated by refining the lateral or vertical discretization of the grid mesh as much as is feasible. The Lake Package computes and lists stage-volume relations for each lake based on the grid discretization specified (unless a user-specified table defining the relation of lake stage to volume and surface area ["lake tables"] are specified for the lake). 
A nonzero lake surface depth can also be used to reduce grid-based lake stage discontinuities for vertical lake connections.

Another consequence of drying all lake-volume grid cells with vertical connections is that lake precipitation and evaporation will not be applied to the lake. This means that the newly dry lake cells should receive the amount of recharge and lose the volume of evapotranspiration at the rates that are specified for the aquifer (specified in the recharge and evapotranspiration packages).

When the lake stage rises, previously dry vertical lake connections may become part of the lake again when the rising lake stage exceeds the elevation of the lake (or the top of the vertically connected GWF Model cell). Lake precipitation and evaporation, not aquifer recharge and evapotranspiration, are once again applied to the vertical lake connections, and the surface area of the GWF Model cell is used once again in lake stage calculations. The Lake Package can also represent the rewetting of a lake after total drying (all vertical lake connections are dry) if there is groundwater inflow into the lake.

\section{Assumptions and Limitations}

A major assumption of the Lake Package is that the lake volume is defined by the lake stage and vertical lake-aquifer connections, unless lake bathymetry is specified for a lake. Because the horizontal cell area is used to define the lake volume, the lateral and vertical grid dimensions must be appropriately chosen so that the spatial extent and bathymetry of the lake are defined with the necessary accuracy. In some cases this may require a finer horizontal discretization in the vicinity of the lake and a finer vertical discretization than would be necessary to simulate heads in the aquifer. Another major assumption is if the head in the aquifer drops below the bottom of a lake still containing water, the seepage rate from the lake is limited to that which would occur if the aquifer head were the same as the elevation of the bottom of the lake. A final assumption is that the lake stage is uniform across the entire surface of the lake, and therefore the lake package is not suitable for simulating lakes that have significant stage changes across the lake.

Lake-aquifer simulations may experience stability problems if inappropriate parameter values are specified in the input data for the setup of the wet-dry option. During a time step in which a dry lake rewets, the use of relatively small time steps can help to avoid substantial inaccuracies in the lake and aquifer water budgets. The method for rewetting a dry lake does not take into account the possible retarding effect of the lakebed, unless the UZF Package is used under the lake.

\section{Incorporation of Leakage Into the Groundwater Flow Equation}

After solution of the continuity equation for each lake, calculated leakage is incorporated into the CVFD flow equation (eq. 2-24) based on whether the standard or Newton-Raphson formulation is being used. The details of how LAK Package leakage is incorporated into the CVFD flow equation is described below.

\section{Standard Formulation}

At the start of each iteration, terms representing lake seepage are added to the flow equation for each lake connection. The choice of which lake seepage equation to use is made by comparing the most recent value of head at the cell to the value of $L B O T_{n b}$ for the reach and the sum of available sources and sinks for a lake. The sum of available sources and sinks for a lake is calculated as

$$
Q S R C_{l}=Q I N F_{l}+\sum_{\substack{n o=1 \\ n o_{d s} \in l}}^{\text {noutlets }} Q O U T L E T_{n o}+Q R O_{l}+Q M V R_{l}+Q P P T_{l}-Q E T_{l}
$$




\section{7-20 Documentation for the MODFLOW 6 Groundwater Flow Model}

Because this process is done at the start of each iteration, the most current value of head $\left(h_{n}\right)$ is the value from the previous iteration. Thus, the check for which lake seepage equation to use lags behind the seepage calculations by one iteration. In cases where the GWF head in cell $n$ is at or below the lake bottom for connection $n b\left(L B O T_{n b}\right)$, lake seepage does not depend on head and is calculated as

$$
\begin{array}{lr}
Q L A K_{n b}=\hat{Q} S R C_{n b} & \text { if } Q L A K_{n b}>\hat{Q} S R C_{n b} \\
Q L A K_{n b}=C L A K_{n b}\left(H L A K_{n b}-L B O T_{n b}\right) & \text { if } Q L A K_{n b} \leq \hat{Q} S R C_{n b},
\end{array}
$$

where $\hat{Q} S R C_{n b}$ is the available water in the lake considering (1) all external lake sources, (2) all aquifer seepage to the lake, and (3) lake seepage to the aquifer for all cells connected to lake $l$ evaluated prior to lake connection $n b$. Because LAK leakage is not head dependent in this case, $Q L A K_{n b}$ is subtracted from the righthand side of equation 2-24. $\hat{Q} S R C_{n b}$ for lake connection $n b$ is calculated as

$$
\hat{Q} S R C_{n b}=Q I N F_{l}+Q R O_{l}+Q M V R_{l}+Q P P T_{l}-\sum_{\substack{i=1 \\ i \in l}}^{n c o n n} Q L A K I_{i}-\sum_{\substack{i=1 \\ i \in l}}^{n b-1} Q L A K O_{i}
$$

For cases where the GWF head in cell $n$ is above the bottom of the lake $\left(L B O T_{n b}\right)$ and the lake leakage to the aquifer is less than the sum of available sources for a lake $\left(\hat{Q} S R C_{n b}\right)$, the term $C L A K_{n b}$ is added to the left-hand side of equation 2-24 and the term $C S F R_{n b} \times H L A K_{n b}$ is subtracted from the right-hand side of equation 2-24. For cases where the GWF head in cell $n$ is above the bottom of the lake and the lake leakage to the aquifer exceeds the sum of available sources for a lake, $\hat{Q} S R C_{n b}$ is subtracted from the right-hand side of equation 2-24.

\section{Newton-Raphson Formulation}

Lake Package boundaries can transition from head dependent to specified flow when $h_{n}<L B O T_{n b}$; finite differences are used to calculate the derivatives. The derivatives are calculated as

$$
\frac{\partial Q L A K_{n b}}{\partial h_{n}}=\frac{Q L A K_{n b}^{+}-Q L A K_{n b}}{\epsilon_{h}}
$$

where $Q L A K_{n b}^{+}$is the lake boundary leakage to GWF node $n$ at $h+\epsilon_{h}$ and $\epsilon_{h}$ is a small perturbation value. For cases where a lake boundary is head dependent $\left(h_{n}+\epsilon_{h}<L B O T_{n b}\right)$, the resulting Newton-Raphson linear equation is

$$
\frac{\partial Q L A K_{n b}}{\partial h_{n}} h_{n}^{k}=-Q L A K_{n b}+\frac{\partial Q L A K_{n b}}{\partial h_{n}} h_{n}^{k-1} .
$$

For head-dependent cases, the terms added to the left- and right-hand side of equation 2-24 as part of the standard formulation are manipulated to formulate the Newton-Raphson equations. The additional operations include: (1) subtracting $C L A K_{n b}$ from the left-hand side of equation 2-24, (2) subtracting the term $C L A K_{n b} \times H L A K_{l}$ from the right-hand side of equation 2-24 to complete the residual $\left(Q L A K_{n b}\right)$, (3) addition of $\frac{\partial Q L A K_{n b}}{\partial h_{n}}$ to the left-hand side of equation 2-24, and (4) addition of $\frac{\partial Q L A K_{n b}}{\partial h_{n}} \times h_{n}^{m-1}$ to the right-hand side of equation 2-24. Equation 2-24 is not modified for the Newton-Raphson formulation unless the leakage rate depends on the groundwater head. 


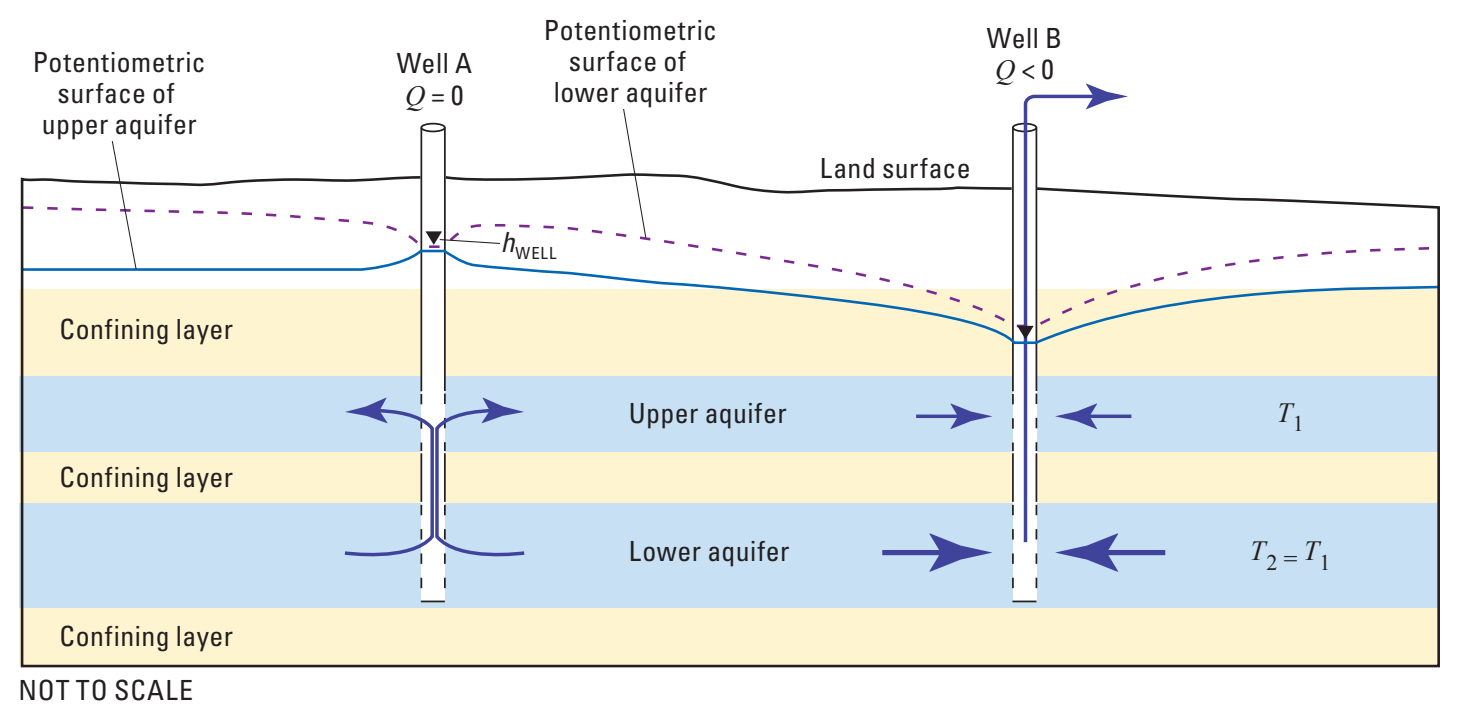

Figure 7-9. Diagram of a cross section showing flow patterns that can be induced by a multi-aquifer well. Well $A$ is a nonpumping well, well $\mathrm{B}$ is a pumping well, $T$ is transmissivity, $H M A W$ is the water level in the well, and $Q$ is discharge rate from the well (in MODFLOW convention, discharge has a negative sign). Figure adapted from Halford and Hanson (2002) and Konikow and others (2009).

\section{Multi-Aquifer Well Package}

The Multi-Aquifer Well (MAW) Package uses the continuity equation to simulate the effect of groundwater pumping on water levels in pumping wells open to multiple aquifers. Wells that are open to multiple aquifers can provide preferential pathways that short circuit normal groundwater flow paths.

The effect of groundwater pumping on multiple aquifers connected by wells can be simulated using the standard well (WEL) Package if the total well pumpage can be manually partitioned by the user prior to the simulation (for example, by using eq. 6-15). The MAW Package partitions the total pumping rate for a pumping well among the various nodes connected to the multi-aquifer pumping well on the basis of the head difference between the well and connected aquifers and well conductance. The MAW Package is a simplification of the actual hydrodynamics of flow through a long borehole. A rigorous representation of the flow dynamics within the borehole, such as analyzed by Cooley and Cunningham (1979), is neither developed nor applied. In fact, for simplicity and computational efficiency, it is assumed that there is hydrostatic equilibrium within the borehole (Bennett and others, 1982; Fanchi and others, 1987).

Under the simplifying assumptions of the MAW Package, at any level of the open or screened borehole, the flow between the well and the adjacent porous media would be controlled by the head difference and the hydraulic conductance between the well and the porous medium for any particular location (grid node) where the well and subsurface material are connected. When a well is open to two or more different intervals in which the aquifer heads are different from each other, the well provides a pathway for flow between the aquifers and flow will occur in the borehole, even in a nonpumped well, in response to head gradients in the aquifer (fig. 7-9).

The MAW Package can also be used to simulated groundwater withdrawals from a single cell. In this case, the MAW Package could be used to account for aquifer and well losses. which are not accounted for in the standard Well (WEL) Package. 


\section{7-22 Documentation for the MODFLOW 6 Groundwater Flow Model}

\section{Mathematical Representation of Head-Loss Terms}

Using the general well-loss equation developed by Jacob (1947) and modified by Rorabaugh (1953), the difference between the head in the cell $\left(h_{n}\right)$ and the head in the well ( $\left.H M A W\right)$ for a well open to just one cell can be expressed as

$$
H M A W=h_{n}+L C 1_{n} Q M A W+L C 2_{n} Q M A W+L C 3_{n} Q M A W^{P}
$$

where $L C 1_{n}$ is a linear aquifer-loss coefficient $\left(T / L^{2}\right), Q M A W$ is the flow between cell $n$ and the multiaquifer well $\left(L^{3} / T\right), L C 2_{n}$ is a linear well-loss coefficient $\left(T / L^{2}\right), L C 3$ is a nonlinear well-loss coefficient $\left(T^{P} / L^{2 P}\right)$, and $P$ is the power (exponent) of the nonlinear discharge component of well loss (-). Equation $7-$ 33 mathematically states that the head in a well is equal to the head in the groundwater node in which the well is located $\left(h_{n}\right)$ plus several head-loss terms. The first head-loss term $\left(L C 1_{n} Q M A W\right)$ accounts for head losses in the aquifer resulting from the well having a radius less than the horizontal dimensions of the cell in which the well is located; the second term $\left(L C 2_{n} Q M A W\right)$ accounts for head losses that occur adjacent to and within the borehole and well screen (that is, skin effects); and the third term $\left(L C 3_{n} Q M A W^{P}\right)$ accounts for nonlinear head losses due to turbulent flow near the well. Under the assumption that most pumping wells are reasonably efficient, equation 7-33 is modified to only consider linear loss terms and rearranged in terms of the flow between MAW well $i$ and cell $n$ to

$$
Q M A W=\frac{1}{L C 1_{n}+L C \mathscr{2}_{n}}\left(H M A W-h_{n}\right) .
$$

Equation 7-34 can be simplified to

$$
Q M A W=C_{M A W, n}\left(H M A W-h_{n}\right),
$$

where $C_{M A W, n}$ is the cumulative well-aquifer conductance $\left(L^{2} / T\right)$ and is equal to the term $\frac{1}{L C 1_{n}+L C 2_{n}}$. Equation 7-35 is identical to the groundwater flow equation between two cells (for example, eq. 2-5).

\section{Aquifer-Loss Coefficient}

A common approach for calculating the aquifer-loss coefficient $(L C 1)$ assumes that aquifer losses can be calculated on the basis of the Thiem (1906) steady-state flow equation, which is

$$
H M A W=h_{n}+\frac{Q M A W}{2 \pi T_{n}} \ln \frac{r_{o_{n}}}{r_{w}}
$$

where $T_{n}$ is the transmissivity of cell $n\left(L^{2} / T\right), r_{o_{n}}$ is the effective (or equivalent) radius of GWF node $n(L)$, and $r_{w}$ is the multi-aquifer well radius $(L)$. Based on Chen and Zhang (2009), the effective radius for cell $n$ is defined as

$$
r_{o_{n}}=\sqrt{\frac{A_{n}}{8 \pi}}
$$




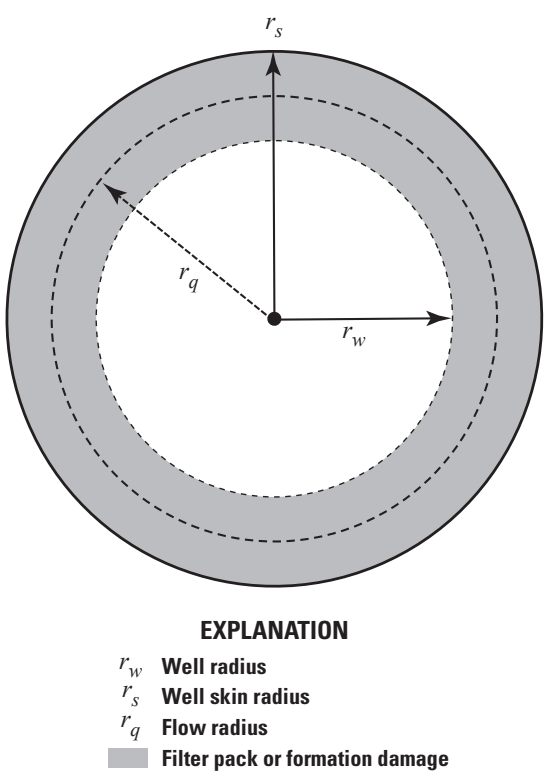

Figure 7-10. Diagram of a well annulus (in plan view) showing well geometry and factors affecting the computed well loss.

Equation 7-37 is approximately equivalent to Peaceman (1983) for square ( $\Delta x=\Delta y)$ and rectangular $(\Delta x \neq \Delta y)$ grid cells in isotropic porous media. For general anisotropic conditions, the transmissivity $(T)$ can be written as

$$
T_{n}=\sqrt{K_{11_{n}} K_{22_{n}}} b_{n},
$$

where $K_{11_{n}}$ is the hydraulic conductivity of the aquifer in the first principal direction for GWF node $n(L / T)$, $K_{22_{n}}$ is the hydraulic conductivity of the aquifer in the second principal direction for GWF node $n(L / T)$, and $b_{n}$ is the saturated aquifer thickness in GWF node $n(L)$ (Konikow and others, 2009). These equations assume that the first and second principal directions for hydraulic conductivity are within the $x, y$ plane. When the aquifer is fully saturated, the resulting aquifer-loss coefficient $\left(L C 1^{0}\right)$ is

$$
L C 1_{n}^{0}=\frac{\ln \frac{r_{o_{n}}}{r_{w}}}{2 \pi T_{n}^{0}}=\frac{\ln \frac{r_{o_{n}}}{r_{w}}}{2 \pi \sqrt{K_{11_{n}} K_{22_{n}}}\left(T O P_{n}-B O T_{n}\right)},
$$

where $T_{n}^{0}$ is the fully saturated transmissivity of cell $n\left(L^{2} / T\right)$. The aquifer-loss coefficient for a given water level in cell $n\left(L C 1_{n}\right)$ is calculated as

$$
L C 1_{n}=\frac{L C 1_{n}^{0}}{S_{M A W, n}^{*}}
$$

where $S_{M A W, n}^{*}$ is the screen saturation in GWF Model cell $n(-)$, which is equal to the GWF Model cell saturation $\left(S_{F_{n}}^{*}\right)$ for conductances calculated using the aquifer- and (or) well-loss coefficients. 


\section{7-24 Documentation for the MODFLOW 6 Groundwater Flow Model}

\section{Well-Loss Coefficient}

The linear well-loss coefficient ( LC2) collectively defines head loss from flow through formation damaged during well drilling, the gravel (filter) pack, and the well screen (fig. 7-10). The coefficient $L C 2$ can be formulated using a dimensionless skin coefficient ( $S K I N)$, as defined by Earlougher (1977) and Halford and Hanson (2002). The skin coefficient represents a zone of affected hydraulic properties close to the wellbore or well screen and is defined as

$$
\operatorname{SKIN}_{n}=\left(\frac{\sqrt{K_{11_{n}} K_{22_{n}}}\left(\operatorname{TOP}_{n}-\mathrm{BOT}_{n}\right)}{K_{s} b_{w}^{0}}-1\right) \ln \left(\frac{r_{s}}{r_{w}}\right)
$$

where $K_{s}$ is the hydraulic conductivity of the filter pack or damaged formation $(L / T), b_{w}^{0}$ is the total screen length in GWF node $n\left(b_{w}^{0}=T O P_{n}-B O T_{n}\right.$ for a fully penetrating well) $(L)$, and $r_{s}$ is the well skin radius $(L)$. The variables $K_{s}$ and $r_{s}$ are rarely known and typically during the model calibration one of these values is fixed and the other is adjusted to match observed data. When the aquifer is fully saturated, the resulting wellloss coefficient $\left(L C 2^{0}\right)$ is

$$
L C 2_{n}^{0}=\frac{S K I N_{n}}{2 \pi T_{n}^{0}}=\frac{S K I N_{n}}{2 \pi \sqrt{K_{11_{n}} K_{22_{n}}}\left(\text { TOP }_{n}-B O T_{n}\right)} .
$$

The well-loss coefficient for a given water level in cell $n\left(L C 2_{n}\right)$ is calculated as

$$
L C 2_{n}=\frac{L C 2_{n}^{0}}{S_{M A W, n}^{*}}
$$

\section{Aquifer- and Well-Loss Coefficient Conductance}

The cumulative well conductance for cell $n$ under saturated conditions that considers aquifer- and wellloss coefficients is

$$
C_{M A W, n}^{0}=\frac{1}{L C 1_{n}^{0}+L C 2_{n}^{0}}
$$

The conductance used to calculate the flow to a multi-aquifer well open to cell $n$ (eq. 7-35) is

$$
C_{M A W, n}=S_{M A W, n}^{*} C_{M A W, n}^{0}
$$

In the case where the aquifer-loss coefficient (eq. 7-40) is the only term considered, the saturated cumulative well conductance for a multi-aquifer well is

$$
C_{M A W, n}^{0}=\frac{1}{L C 1_{n}^{0}}
$$


Similarly, when the well-loss coefficient (eq. 7-43) is the only term considered, the saturated cumulative well conductance for a multi-aquifer well is

$$
C_{M A W, n}^{0}=\frac{1}{L C 2_{n}^{0}}
$$

\section{Alternative Mathematical Representation of Head-Loss Terms}

The aquifer- and well-loss terms (eqs. 7-40 and 7-43) described above are derived for a single well located at the center of a rectangular cell where radial symmetry is not disturbed by hydrologic boundaries or horizontal and vertical heterogeneity (Bennett and others, 1982). Application of these loss terms to more complex problems can be difficult and is dependent on being able to accurately define $r_{o_{n}}$ and $S K I N_{n}$. The work of Kuniansky and Hillestad (1980) and Chappelear and Williamson (1981) provides a basis for developing problem-specific estimates of loss terms for more complex problems. However, loss terms developed using these approaches would be problem specific or applicable to specific types of problems (for example, mutually interfering wells, multiple wells in a single cell, and so forth).

An alternative approach for formulating head-loss terms is to calculate the well conductance using geometric information for a well or directly specifying the well conductance. Well conductance calculated from well construction information for a fully saturated screen in node $n$ is

$$
C_{M A W, n}^{0}=\frac{K_{s} 2 \pi r_{q} b_{w}^{o}}{r_{s}-r_{w}}
$$

where $r_{q}$ is the flow radius ( $L$ ), which is the average of $r_{w}$ and $r_{s}$, and is shown graphically in figure 7-10. Equation 7-48 includes skin effects but does not include aquifer losses resulting from the effective radius for cell $n$ (eq. 7-40). The conductance used to calculate the flow to a multi-aquifer well open to cell $n$ (eq. 7-35) is

$$
C_{M A W, n}=S_{M A W, n}^{*} C_{M A W, n}^{0} .
$$

\section{General Equations for Wells Connected to Multiple Grid Cells}

The total flow rate for multi-aquifer well $i$ connected to multiple GWF cells is calculated as

$$
Q M A W_{i}=\sum_{n \in M A W_{i}} C_{M A W, n}\left(h_{n}-H M A W_{i}\right)
$$

where $Q M A W_{i}$ is the total flow for multi-aquifer well $i\left(L^{3} / T\right)$ and is calculated using the following: well conductance for every cell connected to the multi-aquifer well $\left(n \in M A W_{i}\right)$, the groundwater head for every cell connected to the multi-aquifer well $\left(h_{n}\right)$, and the head in the multi-aquifer well.

\section{Flowing Wells}

In nonpumped open boreholes, if the head in the well is above land surface or the top of the casing (control elevation), water should discharge from the aquifer and flow out of the borehole. The flow rate should be proportional to the head difference between the well and the control elevation. Thus, the discharge would 


\section{7-26 Documentation for the MODFLOW 6 Groundwater Flow Model}

decrease and eventually cease if the head in the aquifer at or near the well declines over time. Head loss in the well is a result of friction losses as water moves up the borehole and energy is lost at constrictions and bends in the borehole. Assuming head losses can be conceptualized as a resistance (the reciprocal of conductance) term, discharge from a flowing well can be calculated as

$$
Q F W_{i}=C F W_{i}\left(T O P_{i}-H M A W\right),
$$

where $Q F W_{i}$ is the flowing well discharge for multi-aquifer well $i\left(L^{3} / T\right), C F W_{i}$ is the flowing well conductance $\left(L^{2} / T\right)$, and $T O P_{i}$ is the top elevation of the flowing well $(L)$. The top elevation of the flowing well is typically equal to the top of the well casing or control elevation. When the water level in the multi-aquifer well is less than $T O P_{i}$ the $C F W_{i}$ is zero and flowing well discharge is zero.

\section{Constraints}

The range over which the water level in a well can potentially change may be restricted by physical or operational constraints. For example, pumping in a withdrawal well cannot continue if the water level drops below the depth of the pump intake and the screened or open-hole intervals. In recharging wells, the water level might be constrained by the land surface or the maximum injection head. Halford and Hanson (2002) recognized that a drawdown (or water-level) constraint on pumping or injection rates is especially useful for predictive scenarios and groundwater management analyses where the future stresses and hydraulic interference among wells are not known, and as a result, this functionality has been included in the multi-aquifer package. Halford and Hanson (2002) also state that the maximum withdrawal rate for an individual well may be limited by the drawdown (change in head or water level) within that well, which is a function of the hydraulic conductivity of the surrounding aquifer, frictional energy loss owing to formation damage from drilling, and energy losses due to flow through the well screen. Nearby wells also can contribute to the drawdown in a pumped well and thereby additionally limit the discharge from a well. The MAW Package constraints in MODFLOW 6 include the well-head constraint method implemented in the MNW2 Package for MODFLOW-2005 (Konikow and others, 2009) and the automatic flow reduction approach available in the WEL Package for MODFLOW-NWT and MODFLOW-USG. The automatic flow reduction constraint adds stability for some types of problems. The well-head constraint approach is only recommended for problems that need to be compared to MODFLOW-2005 results from the MNW1 (Halford and Hanson, 2002) or MNW2 (Konikow and others, 2009) Packages.

\section{Well-Head Constraints}

The MAW Package can compute a drawdown-dependent decrease in the net multi-aquifer well withdrawal rate (or in net recharge rate for an injection well) if well-head constraints are imposed and if a limiting head in the well is reached or exceeded. Furthermore, if the option to apply well-head constraints is activated, then the user can specify a minimum pumping rate that represents the lower limit of the fixed range of pump capacity for each well (the upper limit is the desired flow). The multi-aquifer well withdrawal rate is reduced to zero if the computed net withdrawal falls below the user-specified minimum pumping rate. Recharging (injection) wells are limited in the same manner but the signs are reversed.

In transient flow systems, it is possible for heads at a particular location in an aquifer to cycle between rising and falling stages. This means that a multi-aquifer well for which well-head constraints caused pumping to cease as water levels fell can have pumping and withdrawal restart if water levels subsequently rise above a user-specified threshold elevation. The absolute value of the user-specified threshold must be different from and greater than the absolute value of the minimum user-specified threshold pumping rate to avoid oscillating 

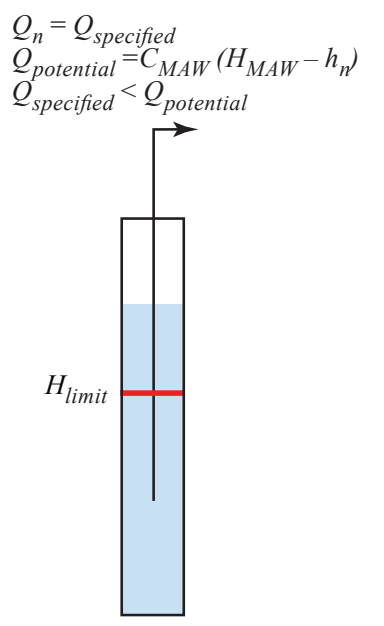

$\frac{\text { Specified }}{\text { Unconstrained }}$
$Q_{n}=Q_{\text {potential }}$
$Q_{\text {potential }}=C_{M A W}\left(H_{\text {limit }}-h_{n}\right)$
$Q_{\text {potential }}<Q_{\text {min }}$

$Q_{n}=0$

$Q_{\text {potential }}=C_{M A W}\left(H_{M A W}-h_{n}\right)$

$Q_{n}=Q_{\text {potential }}$

$Q_{\text {potential }}=C_{\text {MAW }}\left(H_{\text {limit }}-h_{n}\right)$

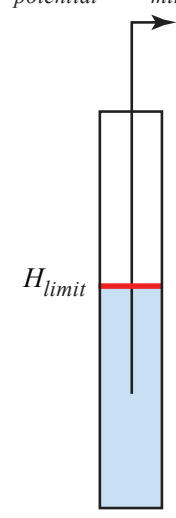

Limited

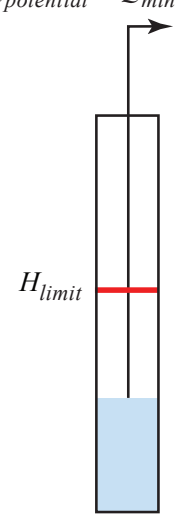

Shutdown

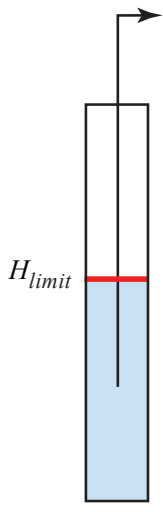

Restart potential $<Q_{\text {restar }}$

Constrained

Figure 7-11. Diagram showing the relation between the computed withdrawal from a multi-aquifer well and the computed head in the well when user-defined well-head constraints are applied. Flow conditions ranging from unconstrained to constrained are shown.

numerical solutions, which could produce instability and lack of convergence in solving the groundwater flow equation.

If the option to impose well-head constraints is activated, then the net withdrawal from a multi-aquifer well becomes limited when the water level in the well reaches or falls below a user-defined lower limit (fig. 711). If the water level in the well remains above or equal to the user-specified threshold head, then the flow rate will be estimated as normal with equation 7-35. Subsequently, if the water level in the well drops below the user-specified threshold head, then the maximum potential multi-aquifer well withdrawal rate is computed using the same equation but with the user-specified threshold head substituted for $H_{M A W}$.

If the potential multi-aquifer well withdrawal rate exceeds the user-specified withdrawal rate, then the latter is used in solving the groundwater flow equation and the well withdrawal rate is not constrained (fig. 7-11). If the potential multi-aquifer well withdrawal rate is less than the user-specified withdrawal rate, then the former is used in solving the groundwater flow equation and the well withdrawal rate is constrained. In this manner, the applicable boundary condition represented by the multinode well transitions from a specified-flow type of boundary condition to a general-head type of boundary condition with $H_{M A W}$ as the controlling head. If $h_{n}$ at all aquifer nodes linked to a multi-aquifer well fall below the user-specified threshold head, then there will be no net withdrawal from the well. Once the pumpage is shut off by well-head constraints during a time step, the multi-aquifer well is not allowed to turn back on during the same time step in order to facilitate stability and convergence of the numerical solution. If the net withdrawal rate from a multi-aquifer well falls to 0 , however, then crossflow between connected cells and intraborehole flow will still be simulated. Recharge (injection) wells are limited in the same manner; however, the signs are reversed and the user-specified threshold head represents a maximum water level.

\section{Automatic Flow Reduction Constraints}

Multi-aquifer well withdrawals can also be constrained using the same approach available in the standard WEL Package (automatic flow reduction) in MODFLOW-NWT and MODFLOW-USG. With this option, 


\section{7-28 Documentation for the MODFLOW 6 Groundwater Flow Model}

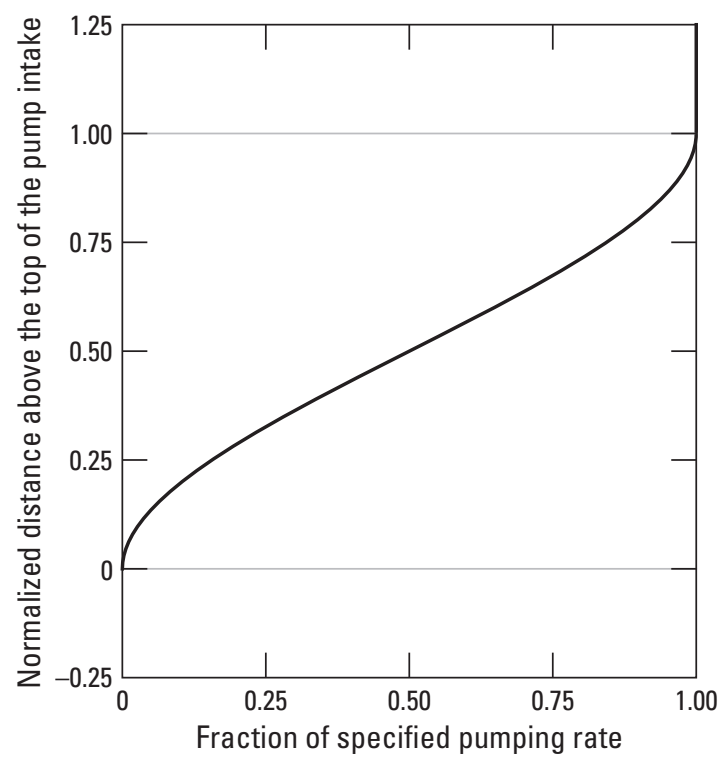

Figure 7-12. Graph showing example of the cubic function used to smoothly reduce specified groundwater withdrawal rates to zero when the water level in a multi-aquifer well is less than a user-specified distance above the top of the pump intake.

when the water level in a well is below a user-specified distance above the top of the specified pump intake elevation, the specified withdrawal rate for a multi-aquifer well is scaled using

$$
Q M A W_{i}=S_{Q M A W_{i}} Q M A W_{i}^{0}
$$

where $Q M A W_{i}$ is the applied multi-aquifer well pumping rate $\left(L^{3} / T\right), S_{Q M A W_{i}}$ is the fraction of the pump rate scaling length above the pump top elevation (-), and $Q M A W_{i}^{0}$ is the user-specified multi-aquifer well pumping rate for well $i\left(L^{3} / T\right)$. If $Q M A W_{i}^{0} \geq 0, S_{Q M A W_{i}}$ is 1 . Otherwise, if $Q M A W_{i}^{0}<0$, then $S_{Q M A W_{i}}$ is defined as

$$
\begin{array}{rrr}
S_{Q M A W_{i}}=b_{i}^{2}\left(\frac{-2}{S C L L E N_{i}^{3}} b_{i}+\frac{3}{S C L L E N_{i}^{2}}\right), & 0<b_{i}<S C L L E N_{i} \\
S_{Q M A W_{i}}=1, & b_{i}>S C L L E N_{i} \\
S_{Q M A W_{i}}=0, & b_{i} \leq 0,
\end{array}
$$

where $b_{i}$ is the difference between the water level in the well $\left(H M A W_{i}\right)$ and the pump top elevation $\left(z_{i}\right)(L)$, and $S C L L E N_{i}$ is the distance above the pump top elevation below which the user-specified multi-aquifer pumping rate is reduced $(L)$. Figure 7-12 shows an example of the smoothing function used to reduce negative multi-aquifer pumping rates to zero. The multi-aquifer well withdrawal rate is set to 0 if the water level in the well is at or below the user-specified pump intake elevation.

\section{Numerical Solution Approach}

The MAW Package adds an additional row to the solution matrix, after the active GWF Model cells, for each MAW Package well for each MAW Package in a GWF Model. The continuity equation is solved for each MAW well and includes contributions from one or more connected GWF Model cells and storage changes. 


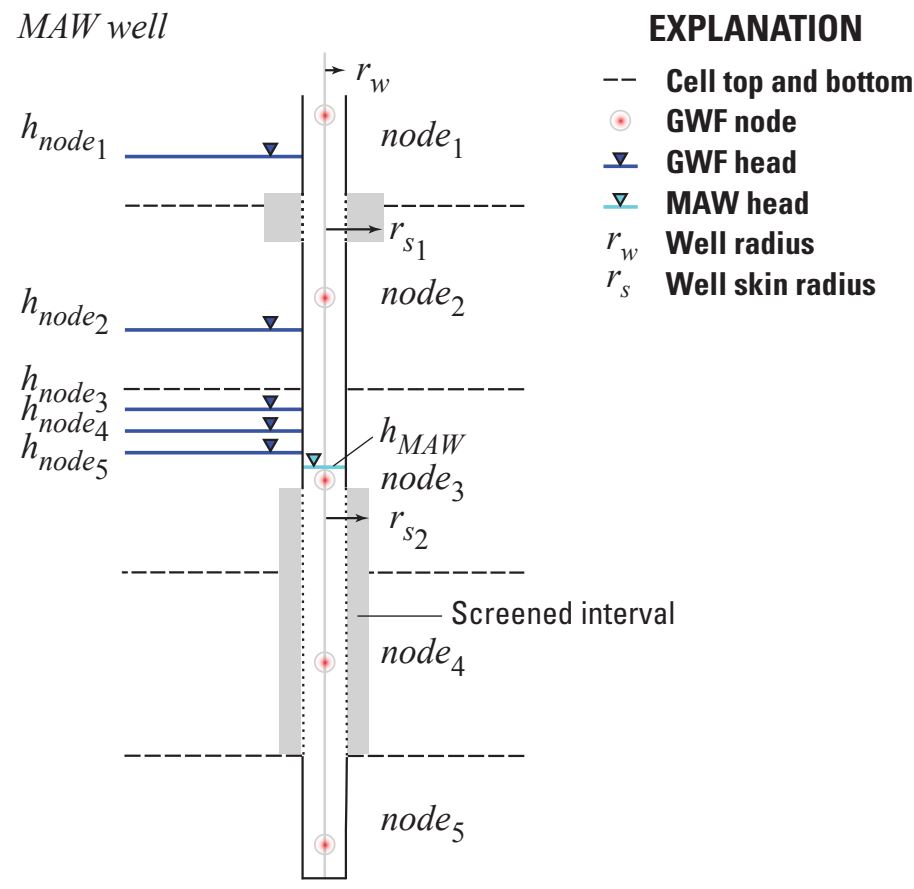

Figure 7-13. Graph showing an example of a multi-aquifer well coupled to multiple model cells, where the well is located at the center of a vertical sequence of model cells.

Off-diagonal terms are added to the continuity equation solved for each GWF Model cell connected to a MAW well.

The MAW Package can be used to simulate groundwater wells that are connected to one or more GWF Model cells (fig. 7-13). Discrete screened intervals can be defined for each connected GWF Model cell and the conductance for each cell is calculated using the saturated thickness of the screen for each connected cell.

The example shown in figure 7-13 is connected to four GWF Model cells. The screen sections connected to GWF Model cells 1, 3, and 4 are fully saturated. The groundwater level in GWF Model cell 2 is below the bottom of the screen and would have a calculated conductance of zero. Although the well extends into GWF Model cell 5 it is not connected to the cell. The radius of each MAW well is constant over the full length of the well but the radius of the skin (filter pack) can be different for each screened interval connected to a GWF Model cell.

Unlike the standard MODFLOW-2005 WEL Package, a continuity equation that incorporates flow between GWF Model cells and the well, well pumpage, well storage changes, and possibly flowing well discharge is solved for each MAW well. Furthermore, well conductance values are incorporated into the GWF Model equations for each MAW connection to a GWF Model cell. 


\section{7-30 Documentation for the MODFLOW 6 Groundwater Flow Model}

Continuity Equation

The continuity equation for each MAW well is

$$
\begin{aligned}
& \sum_{n \in M A W_{i}} S_{M A W, n}^{*} C_{M A W, n}^{0}\left(h_{n}-H M A W_{i}\right)+Q M A W_{i}+ \\
& S_{F W_{i}} C F W_{i}^{0}\left(T O P_{i}-H M A W_{i}\right)=\frac{\pi r_{w}^{2}}{t-t_{\text {old }}}\left(H M A W_{i}-H M A W_{i}^{t_{\text {old }}}\right),
\end{aligned}
$$

where $M A W_{i}$ are the GWF connections for MAW well $i(-), S_{F W_{i}}$ is the fraction of the flowing well scaling length above the top of well $i(-), C F W_{i}^{0}$ is the full flowing well conductance $\left(L^{2} / T\right)$, and $H M A W_{i}^{t_{\text {old }}}$ is the head in MAW well $i$ at the end of the previous time step $(L)$. The fraction of the flowing well scaling length above the top of well $i$ is calculated using the same approach used to scale specified multi-aquifer well pumping rates (eqs. 7-52 and 7-53), with $b_{i}=H M A W_{i}-T O P_{i}$ and $S C L L E N_{i}$ equal to the distance above the top of the well where the user-specified flowing well conductance is scaled.

\section{Incorporation of Multi-Aquifer Well Flow Terms Into the Groundwater Flow Equation}

Multi-aquifer well flow terms are incorporated into the CVFD flow equation (eq. 2-24) based on whether the standard or Newton-Raphson formulation is being used. The details of how the multi-aquifer well flow terms are incorporated into the CVFD flow equation is described below.

\section{Standard Formulation}

Equation 7-54 is rearranged to accumulate terms with head-dependent terms on the left side of the equation and known terms on the right-hand side of the following equation:

$$
\begin{aligned}
& \sum_{n \in M A W_{i}} S_{M A W, n}^{*} C_{M A W, n}^{0}\left(h_{n}-H M A W_{i}\right)-S_{F W_{i}} C F W_{i}^{0} H M A W_{i}-\frac{\pi r_{w}^{2}}{t-t_{\text {old }}} H M A W_{i}= \\
& \quad-Q M A W_{i}-S_{F W_{i}} C F W_{i} T O P_{i}-\frac{\pi r_{w}^{2}}{t-t_{\text {old }}} H M A W_{i}^{t_{\text {old }}} .
\end{aligned}
$$

\section{Newton-Raphson Formulation}

If the Newton-Raphson formulation is being used for MAW wells, then the conductance is upstream weighted. The Newton-Rapshon terms for each MAW well are calculated using analytical derivatives for the GWF Model cell to MAW flow, well storage changes, and flowing well discharge; finite-difference derivatives are used for specified flow rates.

Cell to Well Flow

GWF node to MAW flow can be expressed in general form for upstream weighting as

$$
Q_{M A W, n}=S_{M A W, n}^{*} C_{M A W, n}^{0}\left(h_{n}-H M A W_{i}\right)
$$


The derivatives with respect to $h_{n}$ and $H M A W_{i}$ are obtained from equation 7-56 as

$$
\frac{\partial Q_{M A W, n}}{\partial H M A W_{i}}=-S_{M A W, n}^{*} C_{M A W, n}^{0}+C_{M A W, n}^{0} \frac{\partial S_{M A W, n}^{*}}{\partial H M A W_{i}}\left(h_{n}-H M A W_{i}\right)
$$

and

$$
\frac{\partial Q_{M A W, n}}{\partial h_{n}}=S_{M A W, n}^{*} C_{M A W, n}^{0}+C_{M A W, n}^{0} \frac{\partial S_{M A W, n}^{*}}{\partial h_{n}}\left(h_{n}-H M A W_{i}\right)
$$

Note for upstream weighting that

$$
\frac{\partial S_{M A W, n}^{*}}{\partial H M A W_{i}}= \begin{cases}\frac{\partial S_{M A W, n}^{*}}{\partial H M A W_{i}} & \text { if } M A W \text { is upstream } \\ 0 & \text { if } n \text { is upstream }\end{cases}
$$

and

$$
\frac{\partial S_{M A W, n}^{*}}{\partial h_{n}}= \begin{cases}\frac{\partial S_{M A W, n}^{*}}{\partial h_{n}} & \text { if } n \text { is upstream } \\ 0 & \text { if } M A W \text { is upstream }\end{cases}
$$

The fully implicit form of the Newton-Raphson formulation for horizontal flow between MAW well $i$ and cell $n$ in the form of equation $2-30$ is

$$
\frac{\partial Q_{M A W, n}}{\partial H M A W_{i}} H M A W_{i}^{k}+\frac{\partial Q_{M A W, n}}{\partial h_{n}} h_{n}^{k}=-Q_{M A W, n}^{k-1}+\frac{\partial Q_{M A W, n}}{\partial H M A W_{i}} H M A W_{i}^{k-1}+\frac{\partial Q_{M A W, n}}{\partial h_{n}} h_{n}^{k-1} .
$$

Substitution of equations 7-56, 7-59, and 7-60 into equation 7-61 results in the following general expansion of the Newton-Raphson formulation for horizontal flow between MAW well $i$ and cell $n$ :

$$
\begin{array}{r}
{\left[-S_{M A W, n}^{*} C_{M A W, n}^{0}+C_{M A W, n}^{0} \frac{\partial S_{M A W, n}^{*}}{\partial H M A W_{i}}\left(h_{n}-H M A W_{i}\right)\right] H M A W_{i}^{k}+} \\
{\left[S_{M A W, n}^{*} C_{M A W, n}^{0}+C_{M A W, n}^{0} \frac{\partial S_{M A W, n}^{*}}{\partial h_{n}}\left(h_{n}-H M A W_{i}\right)\right] h_{n}^{k}=} \\
-\left[S_{M A W, n}^{*} C_{M A W, n}^{0}\left(h_{n}^{k-1}-H M A W_{i}^{k-1}\right)\right]+ \\
{\left[-S_{M A W, n}^{*} C_{M A W, n}^{0}+C_{M A W, n}^{0} \frac{\partial S_{M A W, n}^{*}}{\partial M M A W_{i}}\left(h_{n}-H M A W_{i}\right)\right] H M A W_{i}^{k-1}+} \\
{\left[S_{M A W, n}^{*} C_{M A W, n}^{0}+C_{M A W, n}^{0} \frac{\partial S_{M A W, n}^{*}}{\partial h_{n}}\left(h_{n}-H M A W_{i}\right)\right] h_{n}^{k-1} .}
\end{array}
$$




\section{7-32 Documentation for the MODFLOW 6 Groundwater Flow Model}

Simplification of equation $7-62$ results in

$$
\begin{array}{r}
{\left[-S_{M A W, n}^{*} C_{M A W, n}^{0}+C_{M A W, n}^{0} \frac{\partial S_{M A W, n}^{*}}{\partial H M A W_{i}}\left(h_{n}-H M A W_{i}\right)\right] H M A W_{i}^{k}+} \\
{\left[S_{M A W, n}^{*} C_{M A W, n}^{0}+C_{M A W, n}^{0} \frac{\partial S_{M A W, n}^{*}}{\partial h_{n}}\left(h_{n}-H M A W_{i}\right)\right] h_{n}^{k}=} \\
{\left[C_{M A W, n}^{0} \frac{\partial S_{M A W, n}^{*}}{\partial H M A W_{i}}\left(h_{n}-H M A W_{i}\right)\right] H M A W_{i}^{k-1}+} \\
{\left[C_{M A W, n}^{0} \frac{\partial S_{M A W, n}^{*}}{\partial h_{n}}\left(h_{n}-H M A W_{i}\right)\right] h_{n}^{k-1} .}
\end{array}
$$

The $S_{M A W, n}^{*} C_{M A W, n}^{0}$ terms in equation 7-63 were subtracted from the upstream diagonal and added to the appropriate off-diagonal positions of the coefficient matrix during the standard formulation, respectively. The Newton-Raphson formulation is completed by augmenting the coefficient matrix with the second term on the right-hand side of equations 7-59 and 7-60 and adding the product of the second term on the right-hand side of equations $7-59$ or 7-60 and the current upstream head to the right-hand side.

Well Storage Changes

The contribution of the storage to a MAW well $i$ is

$$
Q M A W S_{i}=\frac{\pi r_{w}^{2}}{t-t_{\text {old }}}\left(H M A W_{i}^{t_{\text {old }}}-H M A W_{i}^{k}\right)
$$

where $Q M A W S_{i}$ is the flow resulting from multi-aquifer well storage changes $\left(L^{3} / T\right)$.

The derivative of equation 7-64 with respect to $H M A W_{i}$ is

$$
\frac{\partial Q M A W S_{i}}{\partial H M A W_{i}}=-\frac{\pi r_{w}^{2}}{t-t_{\text {old }}}
$$

The fully implicit form of the Newton-Raphson formulation for storage changes in MAW well $i$ in the form of equation $2-30$ is

$$
\frac{\partial Q M A W_{i}}{\partial H M A W_{i}} H M A W_{i}^{k}=-Q M A W S_{i}+\frac{\partial Q M A W S_{i}}{\partial H M A W_{i}} H M A W_{i}^{k-1} .
$$

Substitution of equations 7-64 and 7-65 into equation 7-66 results in the following general expansion of the Newton-Raphson formulation for the contribution from storage for MAW well $i$ as

$$
\left[-\frac{\pi r_{w}^{2}}{t-t_{\text {old }}}\right] H M A W_{i}^{k}=-\left[\frac{\pi r_{w}^{2}}{t-t_{\text {old }}}\left(H M A W_{i}^{t_{\text {old }}}-H M A W_{i}^{k-1}\right)\right]+\left[-\frac{\pi r_{w}^{2}}{t-t_{\text {old }}}\right] H M A W_{i}^{k-1}
$$


Simplification of equation $7-67$ results in

$$
\left[-\frac{\pi r_{w}^{2}}{t-t_{\text {old }}}\right] H M A W_{i}^{k}=-\left[\frac{\pi r_{w}^{2}}{t-t_{\text {old }}} H M A W_{i}^{t_{\text {old }}}\right]
$$

The terms in equation 7-68 were added to equation 2-24 during the standard formulation. As a result, additional storage terms are not added to equation 2-24 as part of the Newton-Raphson formulation.

Flowing Well Discharge

Flowing well discharge from a multi-aquifer well can be expressed in general form as

$$
Q F W_{i}=S_{F W_{i}} C F W_{i}^{0}\left(T O P_{i}-H M A W_{i}\right)
$$

The derivative of equation 7-69 with respect to $H M A W_{i}$ is obtained from equation 7-69 as

$$
\frac{\partial Q F W_{i}}{\partial H M A W_{i}}=-S_{F W_{i}} C F W_{i}^{0}+C F W_{i}^{0} \frac{\partial S_{F W_{i}}}{\partial H M A W_{i}}\left(T O P_{i}-H M A W_{i}\right)
$$

The fully implicit form of the Newton-Raphson formulation for flowing well discharge from MAW well $i$ in the form of equation $2-30$ is

$$
\frac{\partial Q F W_{i}}{\partial H M A W_{i}} H M A W_{i}^{k}=-Q F W_{i}+\frac{\partial Q F W_{i}}{\partial H M A W_{i}} H M A W_{i}^{k-1}
$$

Substitution of equations 7-69 and 7-70 into equation 7-71 results in the following general expansion of the Newton-Raphson formulation for the contribution from flowing well discharge for MAW well $i$ as

$$
\begin{array}{r}
{\left[-S_{F W_{i}} C F W_{i}^{0}+C F W_{i}^{0} \frac{\partial S_{F W_{i}}}{\partial H M A W_{i}}\left(T_{i}-H M A W_{i}\right)\right] H M A W_{i}^{k}=} \\
-\left[S_{F W_{i}} C F W_{i}^{0}\left(T O P_{i}-H M A W_{i}^{k-1}\right)\right]+ \\
{\left[-S_{F W_{i}} C F W_{i}^{0}+C F W_{i}^{0} \frac{\partial S_{F W_{i}}}{\partial H M A W_{i}}\left(T_{i}-H M A P_{i}\right)\right] H M A W_{i}^{k-1} .}
\end{array}
$$

Simplification of equation $7-72$ results in

$$
\begin{array}{r}
{\left[-S_{F W_{i} C F W_{i}^{0}}+C F W_{i}^{0} \frac{\partial S_{F W_{i}}}{\partial H M A W_{i}}\left(T_{i}-H M A W_{i}\right)\right] H M A W_{i}^{k}=} \\
-\left[S_{F W_{i}} C F W_{i}^{0} T O P_{i}\right]+ \\
{\left[C F W_{i}^{0} \frac{\partial S_{F W_{i}}}{\partial H M A W_{i}}\left(T_{i}-H M A W_{i}\right)\right] H M A W_{i}^{k-1}}
\end{array}
$$

The flowing well conductance $\left(S_{F W_{n}} C F W_{i}^{0}\right)$ terms in equation 7-73 were subtracted from the diagonal of the coefficient matrix in the row for MAW well $i$ during the standard formulation. The product of the flowing well conductance and $T_{O P}$ was subtracted from the right-hand side of equation 2-24 for the row of MAW well 


\section{7-34 Documentation for the MODFLOW 6 Groundwater Flow Model}

$i$ during standard formulation. The Newton-Raphson formulation is completed by augmenting the coefficient matrix with the second term on the right-hand side of equation 7-70 and adding the product of the second term on the right-hand side of equation 7-70 and the current water level in MAW well $i$ to the right-hand side.

Well Flow

The derivative of the well flow is zero unless constraints are specified for a well. If well constraints are not used for a well, the derivative of well flow is zero and additional terms are not added to equation 2-24 as part of the Newton-Raphson formulation. If constraints are used, the well flow derivative, calculated using a finite-difference approximation, is

$$
\frac{\partial Q M A W_{i}}{\partial H M A W_{i}}=\frac{Q M A W_{i}^{+}-Q M A W_{i}}{\epsilon}
$$

where $Q M A W_{i}^{+}$is the specified flow for MAW well $i$ at $H M A W_{i}+\epsilon(L)$.

The fully implicit form of the Newton-Raphson formulation for well discharge from MAW well $i$ in the form of equation $2-30$ is

$$
\frac{\partial Q M A W_{i}}{\partial H M A W_{i}} H M A W_{i}^{k}=-Q M A W_{i}+\frac{\partial Q M A W_{i}}{\partial H M A W_{i}} H M A W_{i}^{k-1}
$$

The variable $Q M A W_{i}$ was subtracted from the right-hand side of equation 2-24-the row for MAW well $i$ during the standard formulation. The Newton-Raphson formulation is completed by adding the right-hand side of equation 7-74 to the diagonal of the coefficient matrix and adding the product of the right-hand side of equation 7-74 and the current water level in MAW well $i$ to the right-hand side. 


\section{Unsaturated Zone Flow Package}

Aquifers often are separated from land surface by an unsaturated zone. Flow through the unsaturated zone is an important process that affects the timing and rates of aquifer recharge. The Unsaturated-Zone Flow (UZF) Package simulates flow through the unsaturated zone and adds the resulting recharge to the CVFD equation for groundwater flow (fig. 7-14). Specified infiltration rates that are greater than the vertical hydraulic conductivity of the unsaturated zone can result in some of the infiltrating water to be rejected. The presence of a shallow water table can also cause infiltration to be rejected. Rejected infiltration and groundwater discharge to the land surface can be routed over land and be instantaneously added to features in other advanced stress packages (SFR, LAK, MAW, and UZF Packages). This instantaneous routing of water to another advanced stress package is handled by the Mover Package, which is described later. The UZF Package simulates evapotranspiration (ET) in both the unsaturated and saturated zones. The residual potential evapotranspiration (PET) demand that is not satisfied by water in the unsaturated zone can be extracted from the saturated zone to simulate groundwater ET. Groundwater ET can be represented using the original linear ET function of Harbaugh (2005), or by a square function described in this section.

Vertical flow through a homogeneous unsaturated zone can be approximated with kinematic waves (Colbeck, 1972; Smith, 1983; Charbeneau, 1984). This approximation is made by simplifying Richards equation, which can be written in the vertical dimension as

$$
\frac{\partial \theta}{\partial t}=\frac{\partial q_{U Z F}}{\partial z}-i_{E T}=\frac{\partial}{\partial z}\left[D(\theta) \frac{\partial \theta}{\partial z}-K(\theta)\right]-i_{E T}
$$

where $\theta$ is the volumetric water content (volume of water per volume of rock $\left.-L^{3} / L^{3}\right), q_{U Z F}$ is the vertical water flux in the unsaturated zone $\left(L^{3} / L^{2} T\right), D(\theta)$ is the hydraulic diffusivity $\left(L^{2} / T\right), K(\theta)$ is the vertical unsaturated hydraulic conductivity as a function of water content $(L / T)$, and $i_{E T}$ is the unsaturated zone ET rate per unit depth $(L / T / L)$. If equation 7-76 is simplified to remove the diffusive term, the vertical flux owing to gravity is

$$
q_{U Z F}=-K(\theta)
$$

where $q_{U Z F}$ is shown as negative downward. Substituting equation 7-77 into equation 7-76, neglecting $D(\theta) \frac{\partial \theta}{\partial z}$ (the diffusive term), and assuming evaporation is removed from the soil profile instantaneously and all flow is downward vertical, yields

$$
\frac{\partial \theta}{\partial t}+\frac{\partial K(\theta)}{\partial z}+i_{E T}=0
$$

Equation 7-78 can be solved by the Method of Characteristics (Abbott, 1966) and results in two ordinary differential equations representing the velocity of a wetting or drying wave and the change in the water content due to ET. The first ordinary differential equation is

$$
\frac{d z}{d t}=\frac{\partial K(\theta)}{\partial \theta}=v(\theta)
$$




\section{7-36 Documentation for the MODFLOW 6 Groundwater Flow Model}
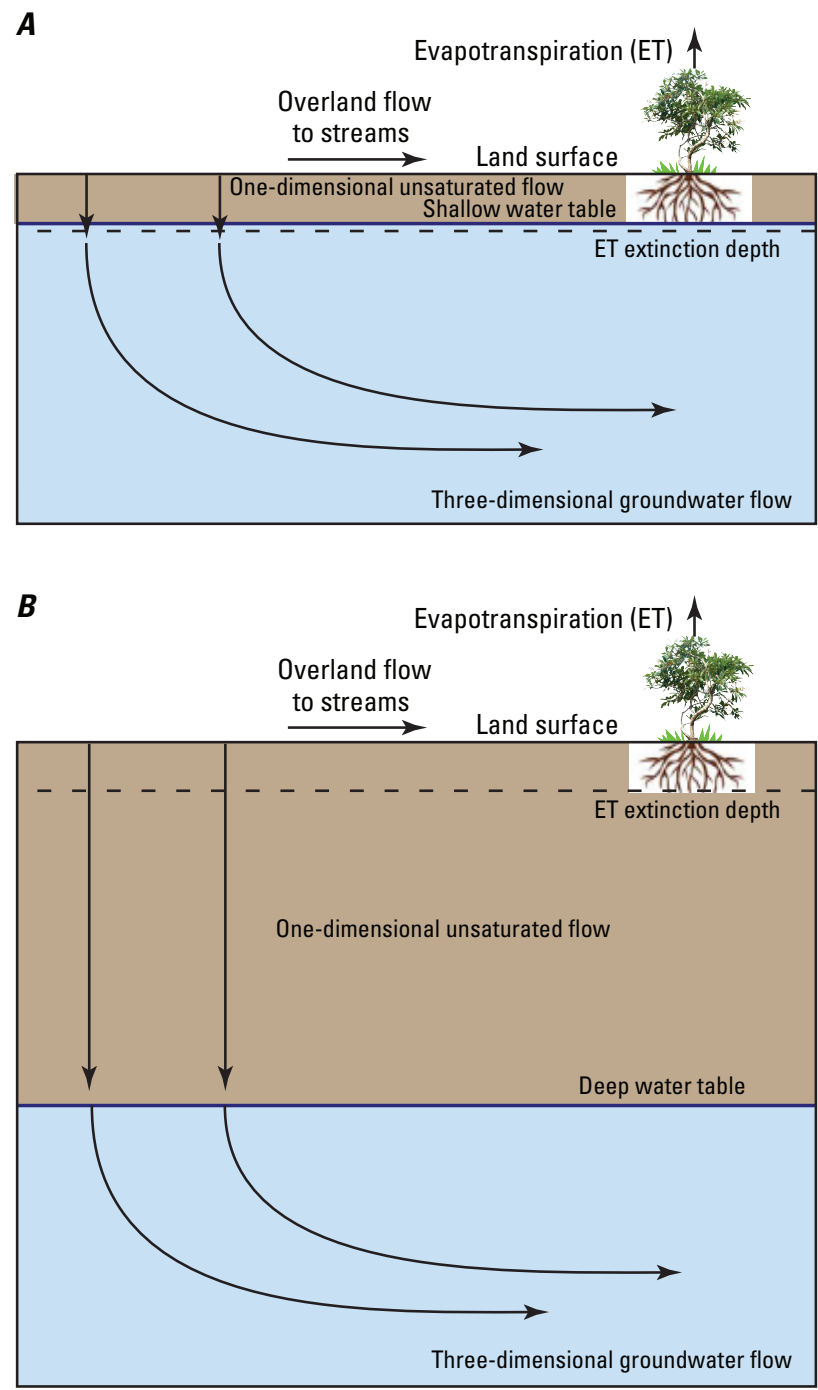

NOT TO SCALE

Figure 7-14. Diagram showing flow through the unsaturated zone. $A$, shallow water table, and $B$, deep water table. Figure modified from Niswonger and others (2006). 
where $v(\theta)$ is the wave velocity restricted to the downward (positive $z$ ) direction $(L / T)$. The second ordinary differential equation is

$$
\frac{d \theta}{d t}=-i_{E T}
$$

This wave represents a wetting front or drying profile in the unsaturated zone that is generated by a sudden increase or decrease in infiltration at land surface, respectively. Integrating equations 7-79 and 7-80 results in

$$
v_{1}=\frac{K\left(\theta_{t_{1}}\right)-K\left(\theta_{t_{0}}\right)}{\theta_{t_{1}}-\theta_{t_{0}}},
$$

where $v_{1}$ is the current wave velocity $(L / T), \theta_{t_{1}}$ is the current volumetric water content of a wave moving through sediment $\left(L^{3} / L^{3}\right)$, and $\theta_{t_{0}}$ is the initial water content of a wave moving through sediment $\left(L^{3} / L^{3}\right)$. The water content along the wetting or drying profile is calculated as

$$
\theta_{t+\Delta t}=-i_{E T} \theta_{t} \Delta t
$$

where $\theta_{t+\Delta t}$ is the current water content along the wetting or drying profile at time $t+\Delta t\left(L^{3} / L^{3}\right)$ and $\theta_{t}$ is the previous water content along the wetting or drying profile at time $t\left(L^{3} / L^{3}\right)$. The Brooks-Corey unsaturated hydraulic conductivity function (Brooks and Corey, 1966) can be used to evaluate $K(\theta)$ and can be expressed as

$$
K(\theta)=K_{\text {sat }}\left[\frac{\theta-\theta_{\text {resid }}}{\theta_{\text {sat }}-\theta_{\text {resid }}}\right]^{\varepsilon},
$$

where $K_{\text {sat }}$ is the saturated hydraulic conductivity $(L / T), \theta_{\text {resid }}$ is the residual (irreducible) water content $\left(L^{3} / L^{3}\right), \theta_{\text {sat }}$ is the saturated water content $\left(L^{3} / L^{3}\right)$, and $\varepsilon$ is the Brooks-Corey exponent (-).

\section{Wetting Fronts and Drainage Profiles}

An increase in the infiltration rate will generate a wetting front, which is represented by a leading wave. A decrease in the infiltration rate will generate a drainage profile, which is represented by a set of trailing waves. Thus, waves are used to represent both wetting fronts and drainage profiles (fig. 7-15). In contrast to a wetting front that stays sharp due to gravity, a drainage profile elongates with time due to gravity. Consequently, a drainage profile is divided into a set of trailing waves that are routed independently at different velocities. Attenuation of a leading wave occurs as a trailing wave overtakes it. When a trailing wave overtakes a leading wave, the water content of the leading wave decreases to the water content of the trailing wave, resulting in drainage. Conversely, when a leading wave overtakes a trailing wave, the leading wave progresses downward, resulting in rewetting. Waves overtake each other due to variations in wave velocity, owing to variations in water content and the effects of gravity.

Trailing waves divide the drainage profile into a set of waves. Equation 7-81 cannot be used to calculate the velocity of a trailing wave because trailing waves do not have a discontinuous front, and thus, the velocity 


\section{7-38 Documentation for the MODFLOW 6 Groundwater Flow Model}

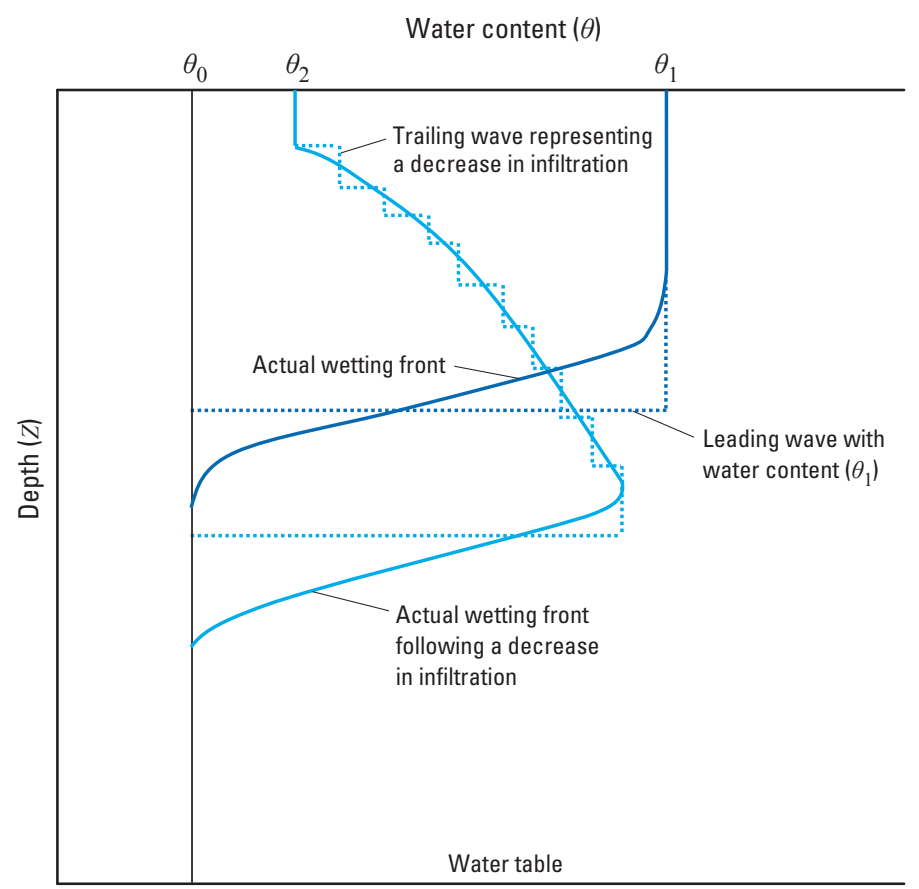

Figure 7-15. Diagram showing wetting fronts and drainage profiles in the unsaturated zone. A wetting front is shown advancing between time $t_{0}$ and time $t_{1}$ and a drainage profile is shown advancing between time $t_{1}$ and time $t_{2}$. Figure modified from Smith (1983).

of trailing waves is calculated using a finite-difference approximation (Smith, 1983). The trailing wave velocity is calculated as

$$
v_{p}=\frac{K\left(\theta_{p}\right)-K\left(\theta_{p}-\Delta \theta\right)}{\Delta \theta}
$$

where $v_{p}$ is the trailing wave velocity at point $p(L / T), \theta_{p}$ is the water content at point $p\left(L^{3} / L^{3}\right)$, and $\Delta \theta$ is the positive change in water content between point $p$ and a location a small distance below point $p$ along a trailing wave $\left(L^{3} / L^{3}\right)$.

A drainage profile and its representation by a set of trailing waves are depicted in figure $7-15$. Values of $\Delta \theta$ increase when a drainage profile is represented by fewer trailing waves. Equation 7-84 becomes less accurate as $\Delta \theta$ increases, or as the drainage profile is represented by fewer trailing waves. A mass balance error of less than 0.05 percent can be achieved with seven to ten trailing waves. The user specifies the number of trailing waves used to represent a drainage profile using input parameter NSTRAIL.

As described, waves are generated by changes in the infiltration rate that result in differing wave velocities. Determination of the timing of wave intersection is required because the wave properties $(v, \theta)$ become discontinuous in time and have to be recalculated to continue the solution. Thus, the UZF Package routinely calculates the shortest time for any two waves to intersect. Waves are routed continuously between wave intersections or intersections between waves and the water table, while wave properties remain constant or change smoothly due to ET. Consequently, the UZF Package requires a unique time discretization that is specific to the problem being solved. The UZF Package time steps are different than those used by MODFLOW. Recharge is calculated at the end of a MODFLOW time step using the time-integrated sum of recharge for all UZF Package subtime steps if subtime steps are required. 


\section{Evapotranspiration in the Unsaturated Zone}

Evaporation and uptake by roots in the unsaturated zone can cause negative potential gradients, such that diffusive forces can be important. Evaporation can cause water to move upward in the unsaturated zone by drying out the soil at land surface or by upward flow from the water table into the capillary zone. These conditions are not modeled by the UZF Package; however, ET from below the water table can be used to represent ET from the capillary zone. Infiltrating water affected by evaporation and uptake by roots can be approximated with kinematic waves. This approach assumes that evaporation and uptake by roots can be grouped together as ET, and that they occur as an instantaneous loss of water over a depth interval equal to the depth of root uptake. ET in the unsaturated zone is specified as a length per time rate $(L / T)$ in the UZF Package and is converted internally to a rate per unit depth by dividing the ET rate by the extinction depth $\left(D_{\text {ext }}\right)$. The extinction water content $\left(\theta_{\text {ext }}\right)$ limits the ET rate if $\theta$ is less than or equal to $\theta_{\text {ext }}$. The value of $\theta_{\text {ext }}$ must be greater than or equal to $\theta_{\text {resid }}$.

ET in the unsaturated zone can also be simulated using a pressure gradient approach as described by Lappala and others (1987). For this case, the capillary pressures are calculated between land surface and $D_{\text {ext }}$ using the Brooks-Corey retention function and air entry pressure. In this case, the ET rate is calculated using

$$
q_{U Z E T_{p}}=K\left(\theta_{p}\right)\left[\psi\left(\theta_{p}\right)-h_{\text {root }_{n b}}\right],
$$

where $q_{U Z E T_{p}}$ is the unsaturated zone evapotransporation for UZF boundary at point $p\left(L^{3} / T\right), \psi(\theta)$ is sediment capillary pressure in UZF boundary $n b$ at point $p(L)$, and $h_{r o o t}$ is the user-specified negative root pressure for UZF boundary $n b(L)$.

\section{Evapotranspiration in the Saturated Zone}

Groundwater ET is simulated in the UZF Package when the user-specified $D_{\text {ext }}$ value exceeds the depth to water in a cell. The UZF Package first satisfies PET by removing water from the unsaturated zone. If PET is not satisfied by the unsaturated zone, then the residual PET (RPET) is used to set the maximum ET rate for groundwater. There are two approaches for simulating groundwater ET. The first approach is a linear decrease in groundwater ET, which is equivalent to the approach implemented in the ET Package (Harbaugh, 2005).

The second approach represents a square ET function and assumes a constant ET rate over the $D_{\text {ext }}$ and a cubic smoothing curve over a small interval (fig. 7-16). ET in the saturated zone is calculated using

$$
Q U G E T_{n b}=S_{U G E T, n} A_{n} R P E T_{n b}
$$

where $Q U G E T_{n b}$ is the volumetric rate of ET removed from GWF cell $n$ connected to UZF boundary $n b$ $\left(L^{3} / T\right)$ and $S_{U G E T, n}$ is a smoothing function that scales the remaining PET (-). The groundwater ET scaling function, $S_{U G E T, n}$, is calculated as

$$
S_{U G E T, n}=\left\{\begin{array}{cc}
-2 \beta^{3}+3 \beta^{2} & \text { for } 0<\beta<1 \\
1 & \text { for } 1 \leq \beta \\
0 & \text { for } \beta \leq 0
\end{array},\right.
$$




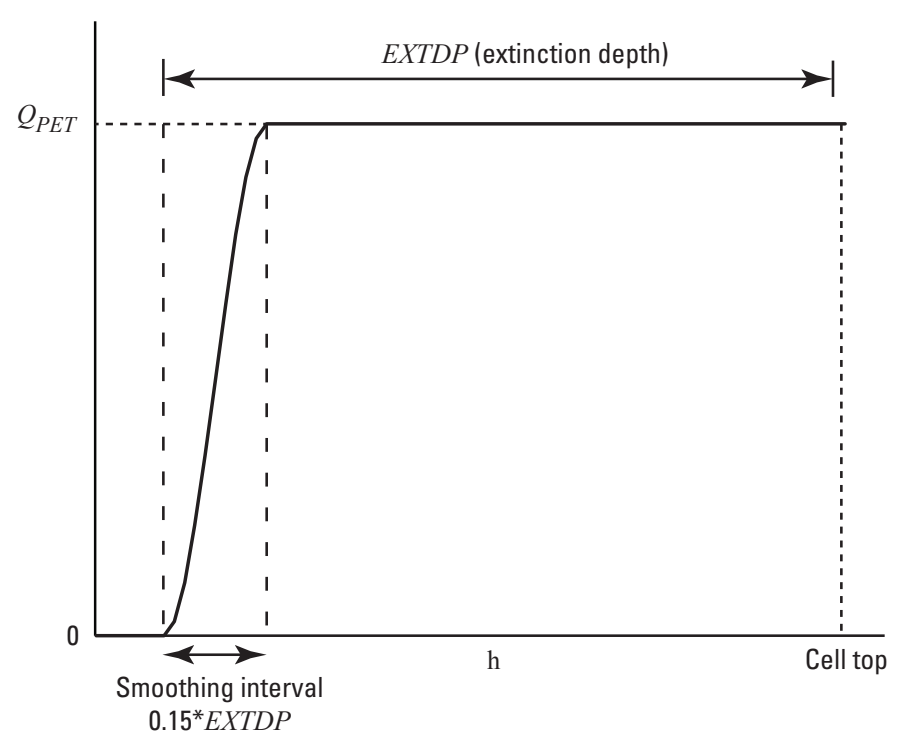

Figure 7-16. Graph showing the evapotranspiration rate as a function of groundwater head for the square wave option.

where $\beta$ is a relative height of the water above $D_{e x t_{n b}}$ for UZF boundary $n b(-)$ and is calculated using

$$
\beta=\frac{h_{n}-z_{n b}}{c},
$$

where $z_{n b}$ is the depth below which smoothing occurs $(L)$. The variable $z_{n b}$ is calculated as

$$
z_{n b}=D_{e x t_{n b}}-c
$$

where $c$ is a smoothing interval above $D_{e x t_{n b}}$, which is equal to $0.15 D_{e x t_{n b}}(L)$.

\section{Infiltration Boundary}

The specified infiltration rate is converted to water content in the UZF Package. The relation between water content and infiltration rate based on equations $7-77$ and $7-83$ is

$$
\begin{array}{rr}
\theta_{q_{a}}=\left(\frac{q_{a}}{K_{\text {sat }}}\right)^{1 / \varepsilon}\left(\theta_{\text {sat }}-\theta_{\text {resid }}\right)+\theta_{\text {resid }} & 0<q_{a} \leq K_{\text {sat }} \\
\theta_{q_{a}}=\theta_{\text {sat }} & K_{\text {sat }}<q_{a},
\end{array}
$$

where $\theta_{q_{a}}$ is the water content of a wave generated from infiltration $\left(L^{3} / L^{3}\right)$ and $q_{a}$ is the volumetric infiltration rate per unit area $\left(L^{3} / L^{2} T\right)$. The water content is set to $\theta_{\text {sat }}$ when the user-specified infiltration rate is equal to, or exceeds, $K_{\text {sat }}$. If the specified infiltration rate is greater than $K_{\text {sat }}$, then the difference between the user-specified infiltration rate and $K_{\text {sat }}$ is multiplied by $A_{n}$ and this volumetric rate of water can be added to a stream, lake, well, or other advanced boundary package using the Mover Package. 


\section{Groundwater Seepage Boundary}

The UZF Package provides an option to simulate groundwater seepage to the land surface (spring discharge) whenever the groundwater head is greater than the land-surface elevation. The volumetric rate of groundwater seepage to land surface is calculated as

$$
Q U G W S_{n b}=S_{U G W S, n} K_{v_{n}} A_{n}\left[\frac{h_{n}-\xi}{0.5\left(T O P_{n}-B O T_{n}\right)}\right]
$$

where $Q U G W S_{n b}$ volumetric rate of groundwater seepage to land surface $\left(L^{3} / T\right), S_{U G W S, n}$ is a smoothing function that scales the groundwater seepage to land surface based on the height of water above land surface in cell $n(-), K_{v_{n}}$ is the vertical hydraulic conductivity of GWF cell $n(L / T)$, and $\xi$ is a user-specified elevation relative to the top of cell $n(L)$. Equation 7-91 assumes that the ponding depth is negligible. An equation for $\xi$ is

$$
\xi=T O P_{n}-0.5 d
$$

where $d$ is a user-specified surface depth (SURFDEP shown in fig. 7-17) (L). Groundwater seepage to land surface is represented with a scaling function, $S_{U G W S, n}$, calculated as

$$
S_{U G W S, n}=\left\{\begin{array}{cc}
-\eta^{3}+2 \eta^{2} & \text { for } 0<\eta<1 \\
1 & \text { for } 1 \leq \eta \\
0 & \text { for } \eta \leq 0
\end{array},\right.
$$

where $\eta$ is the height above land surface relative to $d(-) ; \eta$ is calculated using

$$
\eta=\frac{h_{n}-\xi}{d} .
$$

The derivative of equation 7-91 based on equation 7-93 is used for the Newton-Raphson formulation.

Rejected infiltration occurs when the applied infiltration rate is greater than $K_{\text {sat }}$ or when the groundwater head is at or above land surface. The latter condition is often referred to as saturation-excess rejected infiltration.

Actual infiltration is calculated as

$$
Q U I N F_{n b}=S_{U I N F, n} q_{U I N F} A_{n},
$$

where $Q_{U I N F}$ is the volumetric net infiltration rate for UZF boundary $n b$, which is connected to GWF Model cell $n\left(L^{3} / T\right), S_{U I N F, n}$ is a smoothing function that scales the user-specified infiltration based on the position of the water table relative to land surface in cell $n(-)$, and $q_{U I N F}$ is the minimum of the user-specified infiltration rate $\left(q_{a}\right)$ and $K_{\text {sat }}(L / T)$. The infiltration scaling function, $S_{U I N F, n}$, is calculated as

$$
S_{U I N F, n}=\left\{\begin{array}{cc}
1-\eta & \text { for } 0<\eta<1 \\
0 & \text { for } 1 \leq \eta \\
1 & \text { for } \eta \leq 0
\end{array} .\right.
$$




\section{7-42 Documentation for the MODFLOW 6 Groundwater Flow Model}
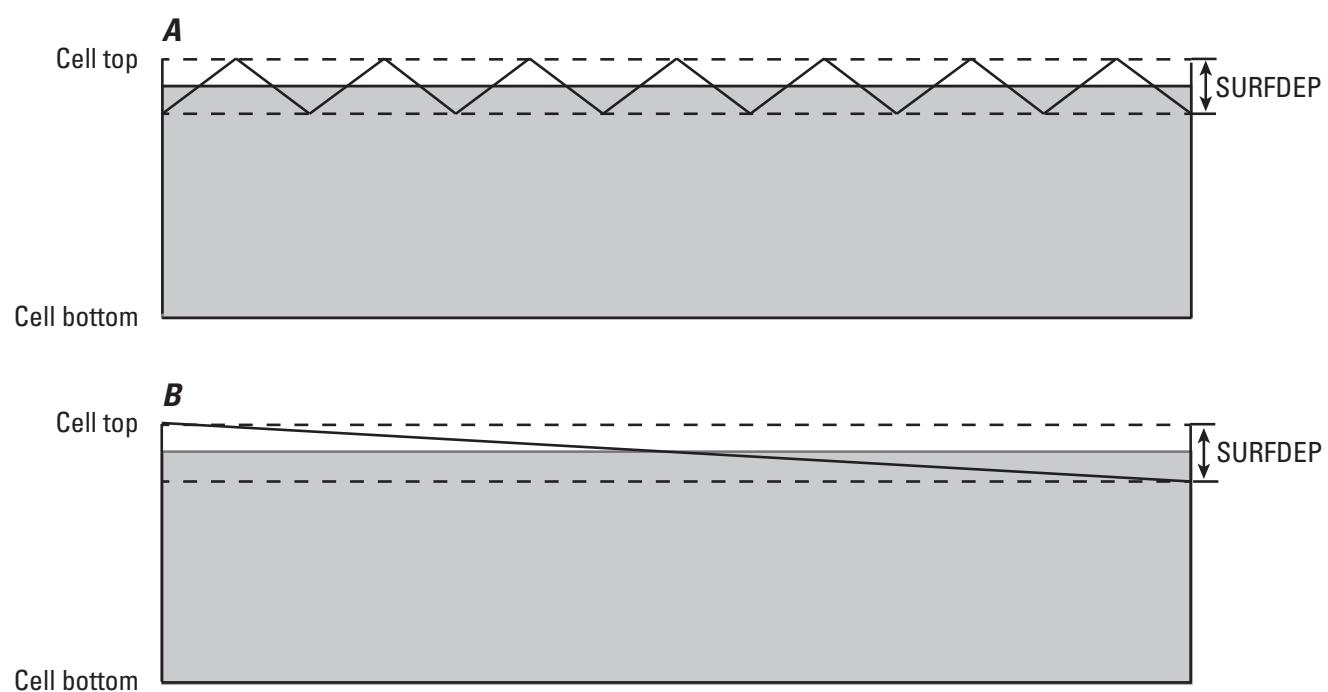

Figure 7-17. Diagram showing two configurations of elevation variations within a model cell that lead to linear relations between infiltration and groundwater head. SURFDEP is the user-specified surface depth. Figure modified from Niswonger and others (2006).

Infiltration reduces linearly to zero as the water table rises to land surface (eq. 7-96). Equation 7-96 is linear and its derivative is zero. Although it is necessary to use a continuous function like equation 7-96 to simulate rejected infiltration, reducing infiltration in this manner also has a physical representation. A model cell is represented by a single cell top $\left(T O P_{n}\right)$; however, there are variations in elevation that occur within a model cell. If variations in elevation within a model cell are linear and symmetric, then infiltration can be assumed to follow a linear relationship with groundwater head, as represented by equation 7-96. Figure 7-17 shows two configurations of elevation variations within a model cell that lead to linear relations between infiltration and groundwater head. Figure 7-18 shows the groundwater discharge and infiltration rates versus different values of $\eta$. Groundwater discharge is smoothed near the zero discharge point for enhanced convergence (fig. 7-18).

\section{Assumptions and Limitations}

A major assumption of UZF is that it does not account for capillary-induced infiltration at the onset of wetting when negative pressure gradients can be large relative to gravity potential gradients. Because negative pressure gradients are ignored, UZF likely underpredicts the infiltration flux and the advancement of the wetting front within hours of the onset of the infiltration event for dry antecedent sediment conditions. However, capillary pressure gradients subside quickly and infiltration due to the force of gravity becomes dominant. Negative pressure gradients also may result in lateral and vertical redistribution in the unsaturated zone, which is not simulated by the UZF Package. The neglect of capillary pressure gradients by the UZF Package likely results in errors in simulated infiltration when the rainfall rate is greater than the saturated vertical hydraulic conductivity and stress periods less than 1 day are used. The UZF Package does not simulate a capillary fringe above the water table and it assumes that groundwater is instantaneously released from or taken into storage.

\section{Incorporation of Terms into the Groundwater Flow Equation}

As discussed above, groundwater ET can be simulated in the UZF Package using a linear or square-wave function. Groundwater ET that uses a linear function is incorporated into the CVFD flow equation (eq. 7-81) using the standard formulation as described in the previous chapter for the Evapotranspiraton Package using a single segment; however, the square-wave function is nonlinear and the derivative of this equation is incorporated into the CVFD flow equation when the Newton-Raphson formulation is specified in the UZF Package. 


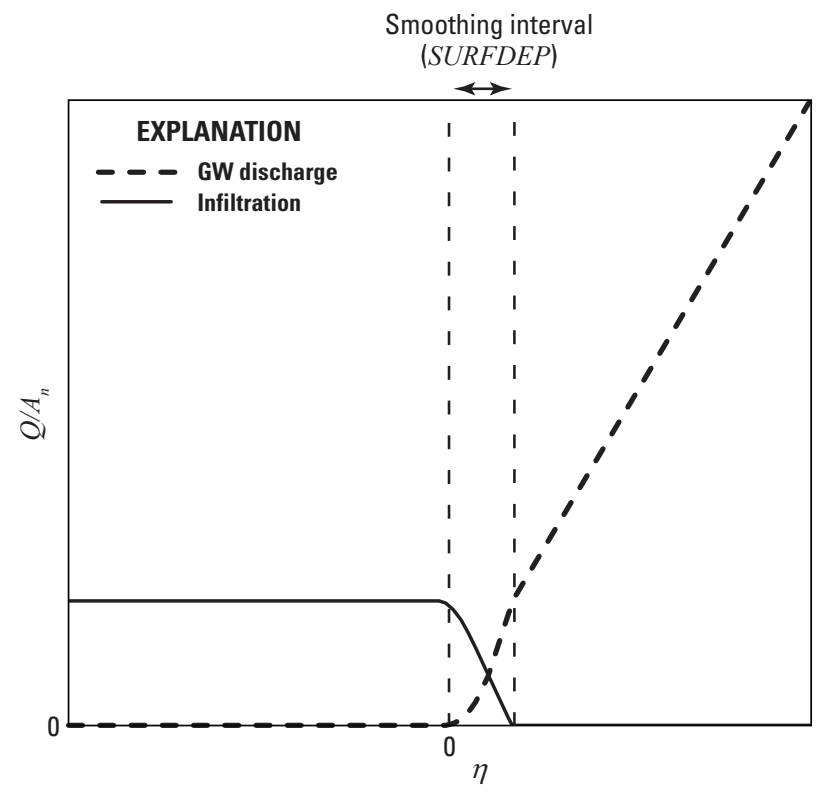

Figure 7-18. Graph showing rates of infiltration and groundwater discharge for different values of $\eta$ and how they vary over the user-specified surface depth, SURFDEP.

The derivative of the ET function (eq. 7-86) with respect to groundwater head is calculated as

$$
\frac{\partial Q U G E T_{n b}}{\partial h_{n}}=\frac{\partial S_{U G E T, n}}{\partial h_{n}} A_{n} R P E T_{n b}
$$

where

$$
\frac{\partial S_{U G E T, n}}{\partial h_{n}}=\left\{\begin{array}{cc}
-6 \beta^{2}+6 \beta & \text { for } 0<\beta<1 \\
0 & \text { for } 1 \leq \beta \\
0 & \text { for } \beta \leq 0
\end{array} .\right.
$$

Infiltration that is routed through the unsaturated zone to the water table is incorporated into the CVFD flow equation using the standard formulation. If the water table rises into the unsaturated zone then recharge is calculated as the sum of water flowing through the unsaturated zone and the volume of water stored in the unsaturated zone submerged by the rising water table. If the water table is equal to or above land surface, then rejected infiltration is calculated as a linear function of groundwater head using the standard formulation.

Groundwater seepage is a nonlinear function of groundwater head and the derivative of equation 7-91 is incorporated into the CVFD flow equation when the Newton-Raphson formulation is specified in the UZF Package. The derivative of the groundwater seepage function is calculated as

$$
\frac{\partial Q U G W S_{n b}}{\partial h_{n}}=\frac{\partial S_{U G W S, n}}{\partial h_{n}} K_{v_{n}} A_{n}\left[\frac{h_{n}-\xi}{0.5\left(T O P_{n}-B O T_{n}\right)}\right]+S_{U G W S, n} K_{v_{n}} A_{n}\left[\frac{1}{0.5\left(T O P_{n}-B O T_{n}\right)}\right],
$$




\section{7-44 Documentation for the MODFLOW 6 Groundwater Flow Model}

where

$$
\frac{\partial S_{U G E T, n}}{\partial h_{n}}=\left\{\begin{array}{cc}
-6 \beta^{2}+6 \beta & \text { for } 0<\beta<1 \\
0 & \text { for } 1 \leq \beta \\
0 & \text { for } \beta \leq 0
\end{array}\right.
$$

\section{Runoff}

The UZF Package includes an option to add runoff generated on a UZF cell to other packages using the Mover Package, which is described in the next section. Runoff generated on a UZF cell can be routed instantaneously to another package, such as a stream, lake, or well. Figure 7-19 shows an example of routing runoff generated on UZF cells to SFR reaches, where SFR reaches receive runoff according to drainage directions defined by slopes in the land-surface elevations. 


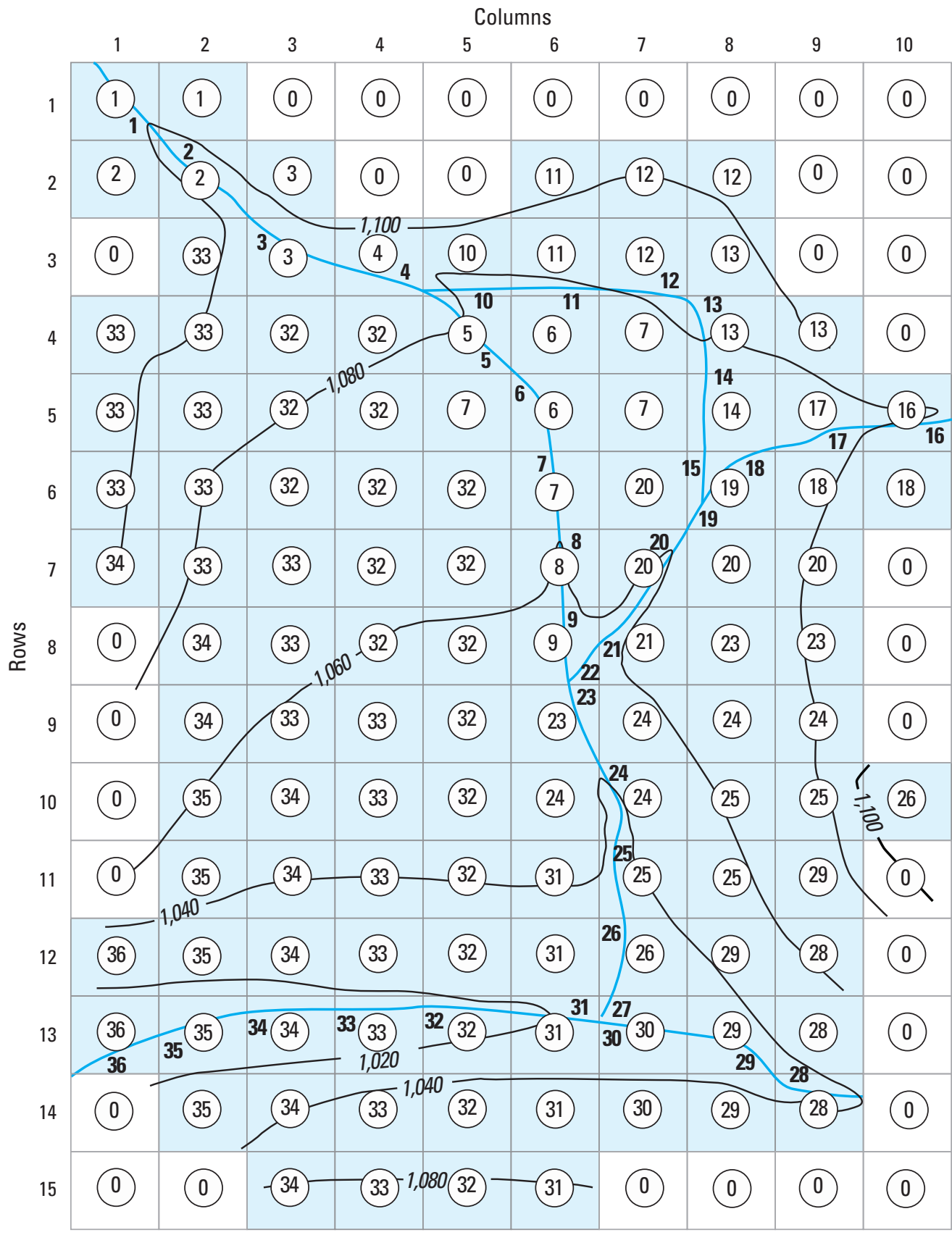

\section{EXPLANATION}

$\begin{array}{cl}\square & \begin{array}{l}\text { Active model cell } \\ \text { Reach number }\end{array} \\ -1,080- & \begin{array}{l}\text { Contour-Shows altitude of land surface } \\ \text { Streams } \\ \text { Reach number assigned to UZF cell }\end{array}\end{array}$

Figure 7-19. Diagram of a model grid showing the Stream-Flow Routing (SFR) Package reach number for each Unsaturated Zone Flow (UZF) cell. Runoff from UZF can be routed to SFR reaches using the Water Mover Package. Figure modified from Niswonger and others (2006). 


\section{7-46 Documentation for the MODFLOW 6 Groundwater Flow Model}

\section{Water Mover Package}

MODFLOW 6 includes a new Water Mover (MVR) Package, which is designed to move water from one package to another package - specifically, water is moved from a feature in one package to a feature in another package. The MVR Package described here is new to MODFLOW and was developed for MODFLOW 6 to meet needs associated with management-based water transfer. The implementation is relatively simple, and allows water to be moved from one package to another package in a general and trackable manner, based on an available amount of water that is dynamically calculated during the simulation. The MVR Package works with all four of the advanced stress packages (MAW, SFR, LAKE, and UZF). Limited functionality is provided for four of the stress packages (WEL, DRN, RIV, and GHB).

\section{Providers and Receivers}

The MVR Package is based on the concept of "providers" and "receivers." The MVR Package transfers available water (or some part thereof) from a provider and applies that water to a receiver. The MVR Package works at the package feature level. A package feature is defined in this section as a single well, drain, stream reach, or lake, for example. It is a single feature of a stress or advanced stress package. The MVR Package can be thought of as an accounting unit that is external to the model. Providers send available water to the MVR Package, and the MVR Package distributes that water to receivers, as requested by the user. No water is transferred if there is no available water or if there are no package features listed to receive the water. The following is a list of package features that can serve as providers:

- Wells in the WEL Package: Available rates of water that can be provided to the MVR Package are well withdrawal rates. In most cases, well withdrawal rates are specified by the user, but if the cell were to go dry, or the automatic flow reduction option were used to decrease the well withdrawal rate, then the available water may be less than the user-specified withdrawal rate. Water injected into the aquifer is not treated as available water by the MVR Package.

- Drains in the DRN Package: Available rates of water that can be provided to the MVR Package are flows into drains from the aquifer.

- Rivers in the River Package: Available rates of water that can be provided to the MVR Package are flows into rivers from the aquifer. Water that flows from rivers into the aquifer is not treated as available water by the MVR Package.

- General-head boundaries in the GHB Package: Available rates of water that can be provided to the MVR Package are flows into general-head boundaries from the aquifer. Water that flows from general-head boundaries into the aquifer is not treated as available water by the MVR Package.

- Multi-aquifer wells in the MAW Package: Available rates of water that can be provided to the MVR Package are well withdrawal rates and flowing well rates. Note that the actual withdrawal rate for the well may or may not be the rate specified by the user. If a cell becomes dry, or if the automatic flow reduction option is used, then the actual well withdrawal rate may not equal the user-specified flow rate. Water injected into the aquifer is not treated as available water by the MVR Package.

- Stream reaches in the SFR Package: Available rates of water that can be provided to the MVR Package are the outflow rates of stream reaches after diversion requirements have been met.

- Lakes in the LAK Package: Available rates of water that can be provided to the MVR Package are outflow rates at lake outlets. 
- Unsaturated zone cells in the UZF Package: Available rates of water that can be provided to the MVR Package are rejected infiltration rates and exfiltration rates.

The list of package features that can receive water from the MVR Package is shorter than the list of providers. The determining factor for whether or not a package feature can be a receiver is whether or not the feature solves a continuity equation. For this reason, individual features of the WEL, DRN, RIV, and GHB Packages cannot be receivers. However, features of the advanced stress packages (multi-aquifer wells, streamflow routing reaches, lakes, and unsaturated zone cells) all solve a continuity equation and can, therefore, receive water from the MVR Package.

\section{Options for Calculating Receiver Flow Rates}

Users have four different options for calculating how much of the available water is provided to the receiver. The available rate of water from the provider is denoted by $Q_{P}$; the rate of water that is applied to the receiver is denoted by $Q_{R}$. For several of the options described below, the user must also specify another rate, $Q_{S}$, which is used in the calculation of $Q_{R}$. These four options (FACTOR, EXCESS, THRESHOLD, and UPTO) are based on the options available in the SFR2 Package (Niswonger and Prudic, 2005) for diverting streamflow. The options are shown here in a different font as they correspond to keywords entered as input to the MVR Package.

1. A FACTOR can be specified such that

$$
Q_{R}=\alpha Q_{P}
$$

where $\alpha$ is the factor to convert the provider flow rate to the receiver flow rate (-).

2. An EXCESS rate can be specified by the user as $Q_{S}$ such that

$$
Q_{R}= \begin{cases}Q_{P}-Q_{S}, & \text { if } Q_{P}>Q_{S} \\ 0, & \text { otherwise }\end{cases}
$$

In the EXCESS case, any water that exceeds the user-specified rate is provided to the receiver. No water is provided to the receiver if the available water is less than the user-specified value.

3. A THRESHOLD rate can be specified for $Q_{S}$ such that

$$
Q_{R}= \begin{cases}0, & \text { if } Q_{S}>Q_{P} \\ Q_{S}, & \text { otherwise }\end{cases}
$$

In the THRESHOLD case, no flow is provided to the receiver until the available water exceeds the userspecified $Q_{S}$ rate. Once the available water exceeds the user-specified rate, then the $Q_{S}$ rate is provided to the receiver. 


\section{7-48 Documentation for the MODFLOW 6 Groundwater Flow Model}

4. An UPTO rate can be specified for $Q_{S}$ such that

$$
Q_{R}=\left\{\begin{array}{ll}
Q_{S}, & \text { if } Q_{P}>Q_{S} \\
Q_{P}, & \text { otherwise }
\end{array} .\right.
$$

In the UPTO case, all of the available water will be taken from the provider up to the $Q_{S}$ value specified by the user. Once $Q_{S}$ is exceeded, the receiver will continue to get the $Q_{S}$ value specified by the user.

For the common situation in which all of the available water is moved from one feature to another, the FACTOR option with an $\alpha$ value of 1.0 is recommended. An example of this type of situation would be the use of a drain to represent groundwater discharge to a spring. In this case, all of the simulated drain flow would be sent directly into a stream. The other three options are useful mostly in controlled and managed contexts, or in situations in which management alternatives need to be evaluated. For example, one may need to capture any streamflow once the streamflow exceeds a certain rate. This could be represented using the MVR Package with the EXCESS option to transfer any flow exceeding the user-specified value in an SFR reach and inject that water into a multi-aquifer well, for example.

The MVR Package is designed so that a package feature can provide water to more than one receiver. The equations shown for the four options calculate $Q_{R}$ based on the water available from the provider, $Q_{P}$. After $Q_{R}$ is calculated, the available water for that package feature is reduced by that amount. The available rate of water continues to be reduced each time the package feature provides water until all of the available water has been consumed. Based on this procedure and the available water, the order of information provided in the MVR Package input file can affect the simulation results if a package feature provides water to more than one other feature. Use of the package in this manner will require the user to consider the order, or priority, for how available water is distributed.

The MVR Package is also designed so that a package feature can receive water from more than one providing features. This makes it possible to inject excess streamflow and excess lake water into a single multiaquifer well, for example. Or one could route the sum of all the drain flow in an area into a single SFR reach. This flexibility makes it possible to route water between many of the packages in ways that allow the actual system behavior to be modeled in a realistic manner. 


\section{Chapter 8. Groundwater Flow Model Exchange}

For some groundwater modeling applications, it may be beneficial to couple two groundwater flow models. There are a variety of situations where coupling groundwater models may be advantageous, such as those shown in figure $8-1$. There may be a need to combine two or more models of an area to better represent the effects of one model on another model (8-1A). Dickinson and others (2007) outlined situations where this may be desirable. There may be a need to couple separate models of individual aquifers, because their effects on one another can no longer be treated through boundary conditions $(8-1 B)$. Feinstein and others (2016) applied a semistructured approach to this type of problem by using a fine grid for the upper model layer and coarser grids for deeper layers. In that case, however, the grids were combined into one unstructured grid and the simulation was performed using MODFLOW-USG. The capability to have locally refined models in selected areas of interest $(8-1 C)$ can also be useful to determine the local and regional effects of pumping wells, for example. This capability is available in the MODFLOW-LGR program (Mehl and Hill, 2013). A final example of model coupling is the use of spatial periodic boundary conditions to represent the effects of repeating features, such as bedforms along the bottom of a stream (Laattoe and others, 2014). In this case, cells on one side of the model can be connected to their corresponding cells on the other side of the model $(8-1 D)$. These are all cases where it may be necessary to connect cells in one model to cells in another model (or the same model) and allow groundwater to flow between them based on a Darcy calculation.

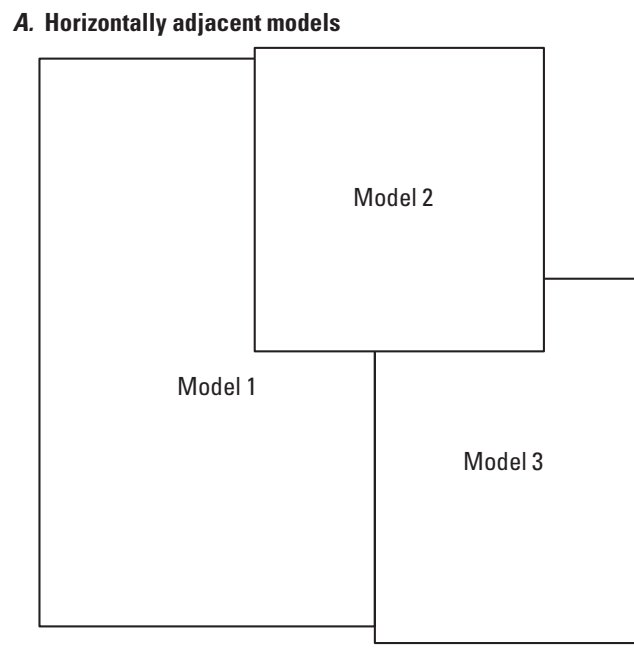

B. Vertically adjacent models
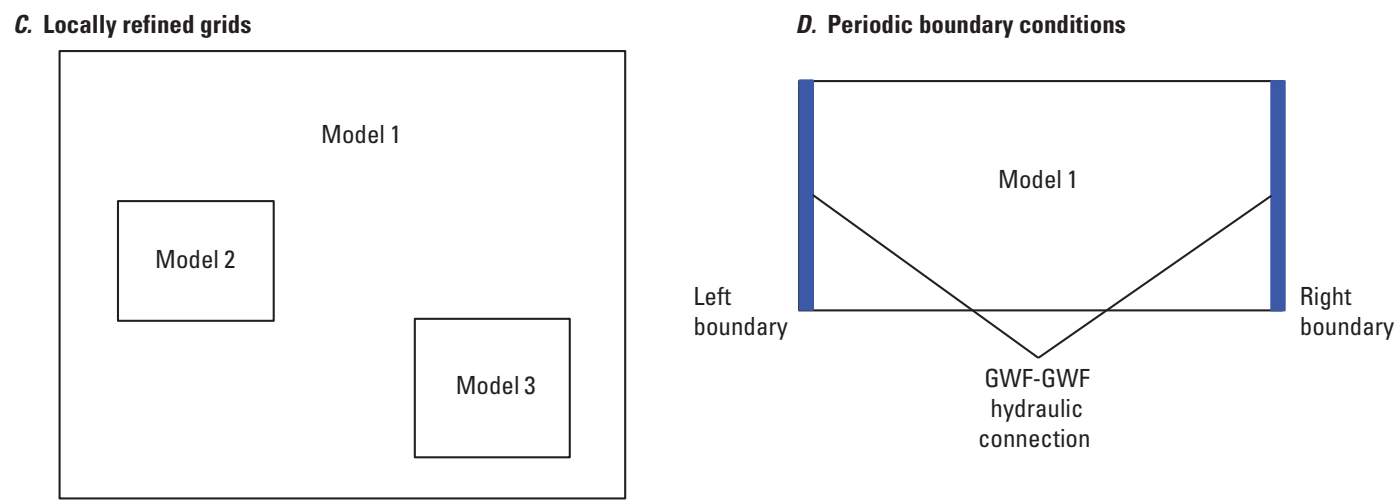

Figure 8-1. Diagram showing model configurations where the Groundwater Flow Exchange (GWF-GWF) may be used to hydraulically connect two different Groundwater Flow Models. $A$, horizontally adjacent models; $B$, vertically adjacent grids; $C$, locally refined grids; and $D$, periodic boundary conditions. 


\section{8-2 Documentation for the MODFLOW 6 Groundwater Flow Model}

\section{Formulation}

A design goal of MODFLOW 6 is to support the tight coupling of multiple models at the matrix level. Coupling at the matrix level, as opposed to a sequential, iterative coupling approach, can result in faster simulation times and will often converge even for problems that do not converge with iteratively coupled models. This chapter describes the GWF Model Exchange object. The GWF Model Exchange object couples two GWF Models, and is therefore specified at the simulation level. A simulation can have as many GWF Model Exchange objects as necessary in order to couple the intended number of models. Multiple GWF Model Exchange objects can also be used to connect two GWF Models. For the LGR case, it may be beneficial to have one GWF Model Exchange object for each face of the child model.

The coupling between models allows groundwater to flow from one model to a connected model using the same flow expression used to calculate groundwater flow between adjacent cells in the same model. Flow between models can be in either direction and may change directions and rate during the simulation in response to changes in hydraulic stresses. Thus, one or more instances of the GWF Model Exchange combined with one or more GWF Models can be used to represent the configurations shown in figure 8-1.

Information about how the models are connected is provided by the user in an input file that is read by the GWF Model Exchange object. The type of information that the user must provide includes the number of cellto-cell connections and geometric properties for those connections. The user must also indicate whether or not the GWF Model Exchange should use the standard formulation or the Newton-Raphson formulation, whether or not ghost node corrections should be applied, and whether or not a water mover will be used to move water between advanced packages in the two connected models.

For each cell-to-cell connection between two GWF models, the user provides information required to calculate the conductance between the two connected cells. This connection information is similar to the following connection information that is required for a GWF Model grid specified using the DISU Package:

- cellid in GWF Model 1 for cell $n$;

- cellid in GWF Model 2 for cell $m$;

- horizontal connection indicator flag $(I H C)$, where 0 is a vertical connection, 1 is a horizontal connection, and 2 is a vertically staggered horizontal connection;

- length $\left(L_{n, m}\right)$ from center of cell $n$ to shared face with cell $m$;

- length $\left(L_{m, n}\right)$ from center of cell $m$ to shared face with cell $n$;

- the horizontal width, $\Delta w_{n, m}$, for a horizontal connection or flow area, $A_{n, m}$, for a vertical connection for the $n-m$ connection; and

- an optional value (provided as an auxiliary variable) that the connection makes with the $x$-axis $\left(\alpha_{n m}\right)$.

This information, when combined with saturated thicknesses and hydraulic conductivity for connected cells in each model, is used to calculate the conductance for the connection. The GWF Model Exchange calculates conductance between two cells in different models in the same way conductance is calculated by the NPF Package for two adjacent cells in a single model. The conductance calculation includes the effect of horizontal anisotropy in hydraulic conductivity if the user provides the angle that the connection makes with the $x$-axis. The GWF Model Exchange also works with the rewetting parameters in each model to determine if a cell in one model should rewet a dry cell in the connected model. 


\section{Ghost Node Correction}

The GNC Package described in chapter 4 can also be used with the GWF Exchange to improve the accuracy of the flow calculation between two connected cells. A GNC Package input file can be provided as an option for each GWF Exchange object. When a GNC Package is provided for a GWF Exchange, then it is assumed that cell $n$ is in the first model and that it contains the ghost node. Cell $m$ is assumed to be in the second model. The cells used for interpolation are also assumed to be in the first model (the model containing cell $n$ ). Thus, for an LGR application, the parent model should be listed first for the simulation, and the child model should be listed in the simulation file as the second model.

\section{Water Mover}

The MVR Package described in chapter 7 can also be used with the GWF Exchange to move water from a package in one GWF Model to an advanced package in another GWF Model. The MVR Package input file can be provided as an option for each GWF Exchange object. When a MVR Package is provided to a GWF Exchange, then the mover package must also include the model name for the provider and the model name for the receiver. When specified for a GWF Exchange, the MVR Package will work according to the description in chapter 7.

\section{Limitations}

There is a limitation with the GWF Model Exchange related to boundary packages that must determine the highest active GWF Model cell. The RCH Package is one example of a package that will apply water to the highest active GWF Model cell. If an entire vertical column of cells is dry in one GWF Model, and recharge is applied to the highest active layer, then the GWF Model Exchange will not pass that recharge quantity to an underlying connected model. This issue is not likely to be encountered for most practical applications. If this issue is encountered, then the user should activate the Newton-Raphson formulation in both models. The Newton-Raphson formulation will pass recharge to the highest active layer. Other packages that apply water to the highest active cell and are susceptible to this issue include the EVT, SFR, LAK, and UZF Packages. 


\section{References Cited}

Abbott, M.B., 1966, An introduction to the method of characteristics: New York, Elsevier Publishing Company, $243 \mathrm{p}$.

Ahlfeld, D.P., Barlow, P.M., and Mulligan, A.E., 2005, GWM-A ground-water management process for the U.S. Geological Survey modular ground-water model (MODFLOW-2000): U.S. Geological Survey OpenFile Report 2005-1072, 130 p., accessed June 27, 2017, at https://pubs.usgs.gov/of/2005/1072/.

Ahlfeld, D.P., Baker, K.M., and Barlow, P.M., 2009, GWM-2005-A Groundwater-Management Process for MODFLOW-2005 with Local Grid Refinement (LGR) capability: U.S. Geological Survey Techniques and Methods, book 6, chap. A33, 65 p, accessed June 27, 2017, at https://pubs.usgs.gov/tm/tm6a33/.

Anderman, E.R., and Hill, M.C., 2000, MODFLOW-2000, the U.S. Geological Survey modular ground-water model-documentation of the Hydrogeologic-Unit Flow (HUF) Package: U.S. Geological Survey Open-File Report 2000-342, 89 p.

Anderman, E.R., and Hill, M.C., 2003, MODFLOW-2000, the U.S. Geological Survey modular ground-water model-Three additions to the Hydrogeologic-Unit Flow (HUF) Package: Alternative storage for the uppermost active cells, flows in hydrogeologic units, and the hydraulic-conductivity depth-dependence (KDEP) capability: U.S. Geological Survey Open-File Report 2003-347, 36 p.

Anderman, E.R., Kipp, K.L., Hill, M.C., Valstar, Johan, and Neupauer, R.M., 2002, MODFLOW-2000, the U.S. Geological Survey modular ground-water model-Documentation of the Model-Layer VariableDirection Horizontal Anisotropy (LVDA) capability of the Hydrogeologic-Unit Flow (HUF) Package: U.S. Geological Survey Open-File Report 02-409, 60 p., accessed June 27, 2017, at https://pubs.er.usgs.gov/ publication/ofr02409.

Anderson, M.P., and Woessner, W.W., 1992, Applied groundwater modeling-Simulation of flow and advective transport: San Diego, Calif., Academic Press, 381 p.

Anderson, M.P., Woessner, W.W., and Hunt, R.J., 2015, Applied groundwater modeling-simulation of flow and advective transport ( $2 \mathrm{~d}$ ed.): London, Academic Press, $630 \mathrm{p}$.

Bakker, Mark, Schaars, Frans, Hughes, J.D., Langevin, C.D., and Dausman, A.M., 2013, Documentation of the seawater intrusion (SWI2) package for MODFLOW: U.S. Geological Survey Techniques and Methods, book 6, chap. A46, 47 p., accessed June 27, 2017, at https://pubs.er.usgs.gov/publication/tm6A46.

Banta, E.R., 2000, MODFLOW-2000, the U.S. Geological Survey Modular Ground-Water Model; documentation of packages for simulating evapotranspiration with a segmented function (ETS1) and drains with return flow (DRT1): U.S. Geological Survey Open File Report 2000-466, 127 p.

Banta, E.R., 2011, MODFLOW-CDSS, a version of MODFLOW-2005 with modifications for Colorado Decision Support Systems: U.S. Geological Survey Open-File Report 2011-1213, 19 p., accessed June 27, 2017, at https://pubs.er.usgs.gov/publication/ofr20111213.

Barkau, R.L., 1996, UNET one-dimensional unsteady flow through a full network of open channels, User's Manual: U.S. Army Corps of Engineers CPD-66, version 3.1, 288 p.

Barrett, Richard, Berry, M.W., Chan, T.F., Demmel, James, Donato, June, Dongarra, Jack, Eijkhout, Victor, Pozo, Roldan, Romine, Charles, and Van der Vorst, Henk, 1994, Templates for the solution of linear systems-Building blocks for iterative methods: Philadelphia, Penn., Society for Industrial and Applied Mathematics, 124 p., accessed June 27, 2017, at https://doi.org/10.1137/1.9781611971538.

Bear, Jacob, 1979, Hydraulics of groundwater: New York, MacGraw-Hill, 567 p.

Bennett, G.D., Kontis, A.L., and Larson, S.P., 1982, Representation of multiaquifer well effects in threedimensional ground-water flow simulation: Groundwater, v. 20, no. 3, p. 334-341, accessed June 27, 2017, at https://doi.org/10.1111/j.1745-6584.1982.tb01354.x. 


\section{R-2 Documentation for the MODFLOW 6 Groundwater Flow Model}

Brooks, R.H., and Corey, A.T., 1966, Properties of porous media affecting fluid flow: American Society of Civil Engineers, Journal of Irrigation and Drainage, v. 101, p. 85-92.

Chappelear, J.E., and Williamson, A.S., 1981, Representing wells in numerical reservoir simulation-Part 2 Implementation: Society of Petroleum Engineers Journal, v. 21, no. 03, p. 339-344, accessed June 27, 2017, at https://doi.org/10.2118/9770-PA.

Charbeneau, R.J., 1984, Kinematic models for soil moisture and solute transport: Water Resources Research, v. 20, no. 6, p. 699-706, accessed June 27, 2017, at https://doi.org/10.1029/WR020i006p00699.

Chaudhry, M.H., 2007, Open-channel flow (2d ed.): New York, Springer Science \& Business Media, 523 p., accessed June 27, 2017, at https://doi.org/10.1007/978-0-387-68648-6.

Chen, Zhangxin, and Zhang, Youqian, 2009, Well flow models for various numerical methods: International Journal of Numerical Analysis and Modeling, v. 6, no. 3, p. 375-388.

Cheng, Xiangxue, and Anderson, M.P., 1993, Numerical simulation of ground-water interaction with lakes allowing for fluctuating lake levels: Ground Water, v. 31, no. 6, p. 929-933, accessed June 27, 2017, at https://doi.org/10.1111/j.1745-6584.1993.tb00866.x.

Colbeck, S.C., 1972, A theory of water percolation in snow: Journal of Glaciology, v. 2, no. 63, p. 699-706, accessed June 27, 2017, at https://doi.org/10.1017/S0022143000022346.

Collins, R.E., 1961, Flow of fluids through porous materials: New York, Reinhold Publishing Corporation, $270 \mathrm{p}$.

Cooley, R.L., and Cunningham, A.B., 1979, Consideration of total energy loss in theory of flow to wells: Journal of Hydrology, v. 43, no. 1, p. 161-184, accessed June 27, 2017, at https://doi.org/10.1016/ 0022-1694(79)90171-9.

Crichlow, H.B., 1977, Modern reservoir engineering-A simulation approach: Englewood Cliffs, N.J., Prentice Hall, Inc., 354 p.

Danskin, W.R., and Hanson, R.T., 2002, Appendix 2-Documentation and description of changes made to the streamflow-routing package in the MODFLOW groundwater flow model, in Hanson, R.T., Martin, P.M., and Koczot, K.M., Simulation of ground-water/surface-water flow in the Santa Clara-Calleguas Basin, Ventura County, California: U.S. Geological Survey Water-Resources Investigations Report 02-4136, p. 187-193, accessed June 27, 2017, at https://pubs.er.usgs.gov/publication/wri024136.

Dehotin, Judicaël, Vázquez, R.F., Braud, Isabelle, Debionne, S., and Viallet, P., 2010, Modeling of hydrological processes using unstructured and irregular grids-2D groundwater application: Journal of Hydrologic Engineering, v. 16, no. 2, p. 108-125, accessed June 27, 2017, at https://doi.org/10.1061/(ASCE)HE. 1943-5584.0000296.

Dickinson, J.E., James, S.C., Mehl, S.W., Hill, M.C., Leake, S.A., Zyvoloski, G.A., Faunt, C.C., and Eddebbarh, Al-Aziz, 2007, A new ghost-node method for linking different models and initial investigations of heterogeneity and nonmatching grids: Advances in Water Resources, v. 30, no. 8, p. 1722-1736, accessed June 27, 2017, at https://doi.org/10.1016/j.advwatres.2007.01.004.

Earlougher, R.C., 1977, Advances in well test analysis: Dallas, Tex., Society of Petroleum Engineers of the American Institute of Mining, 264 p.

Edwards, M.G., 1996, Elimination of adaptive grid interface errors in the discrete cell centered pressure equation: Journal of Computational Physics, v. 126, no. 2, p. 356-372, accessed June 27, 2017, at https: //doi.org/10.1006/jcph.1996.0143.

Fanchi, J.R., Kennedy, J.E., and Dauben, D.L., 1987, BOAST II-A three-dimensional, three-phase black oil applied simulation tool: U.S. Department of Energy Report DOE/BC-88/2/SP, variously paged, accessed July 5, 2016, at https://edx.netl.doe.gov/dataset/0616089d-7e1d-40ce-8396-428356983256. 
Feinstein, D.T., Fienen, M.N., Reeves, H.W., and Langevin, C.D., 2016, A semi-structured MODFLOW-USG model to evaluate local water sources to wells for decision support: Groundwater, v. 54, no. 4, p. 532-544, accessed June 27, 2017, at https://doi.org/10.1111/gwat.12389.

Fenske, J.P., Leake, S.A., and Prudic, D.E., 1996, Documentation of a computer program (RES1) to simulate leakage from reservoirs using the modular finite-difference ground-water flow model (MODFLOW): U.S. Geological Survey Open-File Report 96-364, 51 p., accessed June 27, 2017, at https://pubs.er.usgs.gov/ publication/ofr96364.

Freeze, R.A., and Cherry, J.A., 1979, Groundwater: Englewood Cliffs, N.J., Prentice-Hall, 604 p.

Goode, D.J., and Appel, C.A., 1992, Finite-difference interblock transmissivity for unconfined aquifers and for aquifers having smoothly varying transmissivity: U.S. Geological Survey Water-Resources Investigations Report 92-4124, 79 p., accessed June 27, 2017, at https://pubs.er.usgs.gov/publication/wri924124.

Halford, K.J., and Hanson, R.T., 2002, User guide for the drawdown-limited, multi-node well (MNW) package for the U.S. Geological Survey's modular three-dimensional finite-difference ground-water flow model, versions MODFLOW-96 and MODFLOW-2000: U.S. Geological Survey Open-File Report 02-293, 33 p.

Hanson, R.T., and Leake, S.A., 1999, Documentation for HYDMOD-A program for extracting and processing time-series data from the U.S. Geological Survey's modular three-dimensional finite-difference groundwater flow model: U.S. Geological Survey Open-File Report 98-564, 57 p., accessed June 27, 2017, at https://pubs.er.usgs.gov/publication/ofr98564.

Hanson, R.T., Boyce, S.E., Schmid, Wolfgang, Hughes, J.D., Mehl, S.W., Leake, S.A., Maddock, Thomas, III, and Niswonger, R.G., 2014, One-Water Hydrologic Flow Model (MODFLOW-OWHM): U.S. Geological Survey Techniques and Methods, book 6, chap. A51, 120 p., accessed June 27, 2017, at https://doi.org/10. 3133/tm6A51.

Harbaugh, A.W., 2005, MODFLOW-2005, the U.S. Geological Survey modular ground-water model-the Ground-Water Flow Process: U.S. Geological Survey Techniques and Methods, book 6, chap. A16, variously paged, accessed June 27, 2017, at https://pubs.usgs.gov/tm/2005/tm6A16/.

Harbaugh, A.W., and McDonald, M.G., 1996a, Programmer's Documentation for MODFLOW-96, an update to the U.S. Geological Survey modular three-dimensional finite-difference ground-water flow model: U.S. Geological Survey Open-File Report 96-486, 220 p.

Harbaugh, A.W., and McDonald, M.G., 1996b, User's documentation for MODFLOW-96, an update to the U.S. Geological Survey modular finite-difference ground-water flow model: U.S. Geological Survey Techniques of Water-Resources Investigations, book 6, chap. A1, $586 \mathrm{p}$.

Harbaugh, A.W., Banta, E.R., Hill, M.C., and McDonald, M.G., 2000, User guide to modularization concepts and the Ground-Water Flow Process, MODFLOW-2000 the U.S. Geological Survey modular ground-water model: U.S. Geological Survey Open-File Report 00-92, 121 p., accessed June 27, 2017, at https://pubs.er. usgs.gov/publication/ofr200092.

Hill, M.C., 1990, Preconditioned Conjugate-Gradient 2 (PCG2), a computer program for solving ground-water flow equations: U.S. Geological Survey Water-Resources Investigations Report 90-4048, 25 p., accessed June 27, 2017, at https://pubs.usgs.gov/wri/wrir_90-4048.

Hill, M.C., Banta, E.R., Harbaugh, A.W., and Anderman, E.R., 2000, MODFLOW-2000, the U.S. Geological Survey modular ground-water model-User guide to the observation, sensitivity, and parameter-estimation processes and three post-processing programs: U.S. Geological Survey Open-File Report 00-184, 210 p.

Hoffmann, Jörn, Leake, S.A., Galloway, D.L., and Wilson, A.M., 2003, MODFLOW-2000 Ground-Water Model-User Guide to the Subsidence and Aquifer-System Compaction (SUB) Package: U.S. Geological Survey Open-File Report 03-233, 44 p., accessed June 27, 2017, at https://pubs.usgs.gov/of/2003/ ofr03-233/. 


\section{R-4 Documentation for the MODFLOW 6 Groundwater Flow Model}

Hsieh, P.A., and Freckleton, J.R., 1993, Documentation of a computer program to simulate horizontal-flow barriers using the U.S. Geological Survey's modular three-dimensional finite-difference ground-water flow model: U.S. Geological Survey Open-File Report 92-477, 32 p., accessed June 27, 2017, at https://pubs.er. usgs.gov/publication/ofr92477.

Hughes, J.D., Langevin, C.D., Chartier, K.L., and White, J.T., 2012, Documentation of the Surface-Water Routing (SWR1) Process for modeling surface-water flow with the U.S. Geological Survey modular groundwater model (MODFLOW-2005): U.S. Geological Survey Techniques and Methods, book 6, chap. A40 (Version 1.0), 113 p., accessed June 27, 2017, at https://pubs.usgs.gov/tm/6a40/.

Hughes, J.D., Langevin, C.D., and Banta, E.R., 2017, Documentation for the MODFLOW 6 framework: U.S. Geological Survey Techniques and Methods, book 6, chap. A57, 36 p., accessed August 4, 2017, at https: //doi.org/10.3133/tm6A57.

International Standards Organization, 2004, Information technology-Programming languages-Fortran-Part 1; Base language: Geneva, International Standards Organization, 585 p.

International Standards Organization, 2010, Information technology-Programming languages-Fortran-Part 1; Base language: Geneva, International Standards Organization, 603 p.

Jacob, C.E., 1947, Drawdown test to determine effective radius of artesian well: Transactions of the American Society of Civil Engineers, v. 112, p. 1047-1070.

Keating, Elizabeth, and Zyvoloski, George, 2009, A stable and efficient numerical algorithm for unconfined aquifer analysis: Ground Water, v. 47, no. 4, p. 569-579, accessed June 27, 2017, at https://doi.org/10.1111/ j.1745-6584.2009.00555.x.

Konikow, L.F., Goode, D.J., and Hornberger, G.Z., 1996, A three-dimensional method-of-characteristics solute-transport model (MOC3D): U.S. Geological Survey Water-Resources Investigations Report 96-4267, 87 p., accessed June 27, 2017, at https://pubs.er.usgs.gov/publication/wri964267.

Konikow, L.F., Hornberger, G.Z., Halford, K.J., and Hanson, R.T., 2009, Revised multi-node well (MNW2) package for MODFLOW ground-water flow model: U.S. Geological Survey Techniques and Methods, book 6, chap. A30, 67 p., accessed June 27, 2017, at https://pubs.usgs.gov/tm/tm6a30/.

Kuiper, L.K., 1987, Computer program for solving ground-water flow equations by the preconditioned conjugate gradient method: U.S. Geological Survey Water-Resources Investigations Report 87-4091, 34 p.

Kuniansky, Joseph, and Hillestad, J.G., 1980, Reservoir simulation using bottomhole pressure boundary conditions: Society of Petroleum Engineers Journal, v. 20, no. 06, p. 473-486, accessed June 27, 2017, at https://doi.org/10.2118/8767-PA.

Laattoe, Tariq, Post, V. E.A., and Werner, A.D., 2014, Spatial periodic boundary condition for MODFLOW: Groundwater, v. 52, no. 4, p. 606-612, accessed June 27, 2017, at https://doi.org/10.1111/gwat.12086.

Langevin, C.D., Thorne Jr, D.T., Dausman, A.M., Sukop, M.C., and Guo, Weixing, 2008, SEAWAT Version 4-A computer program for simulation of multi-species solute and heat transport: U.S. Geological Survey Techniques and Methods, book 6, chap. A22, 39 p., accessed June 27, 2017, at https://pubs.er.usgs.gov/ publication/tm6A22.

Lappala, E.G., Healy, R.W., Weeks, E.P., and others, 1987, Documentation of computer program VS2D to solve the equations of fluid flow in variably saturated porous media: U.S. Geological Survey WaterResources Investigations Report 83-4099, 184 p., accessed June 27, 2017, at https://pubs.er.usgs.gov/ publication/wri834099.

Leake, S.A., and Galloway, D.L., 2007, MODFLOW Ground-water model-User guide to the Subsidence and Aquifer-System Compaction Package (SUB-WT) for Water-Table Aquifers: U.S. Geological Survey Techniques and Methods, book 6, chap. A23, 42 p., accessed June 27, 2017, at https://pubs.er.usgs.gov/ publication/tm6A23. 
Leake, S.A., and Lilly, M.R., 1997, Documentation of computer program (FHB1) for assignment of transient specified-flow and specified-head boundaries in applications of the modular finite-diference ground-water flow model (MODFLOW): U.S. Geological Survey Open-File Report 97-571, 50 p., accessed June 27, 2017, at https://pubs.er.usgs.gov/publication/ofr97571.

Leake, S.A., and Prudic, D.E., 1991, Documentation of a computer program to simulate aquifer-system compaction using the modular finite-difference ground-water flow model: U.S. Geological Survey Techniques of Water-Resources Investigations, book 6, chap. A2, 68 p., accessed June 27, 2017, at https://pubs.er.usgs.gov/ publication/twri06A2.

Maddock, Thomas, III, Baird, K.J., Hanson, R.T., Schmid, Wolfgang, and Ajami, Hoori, 2012, RIP-ET—A Riparian Evapotranspiration Package for MODFLOW-2005: U.S. Geological Survey Techniques and Methods, book 6, chap. A39, 76 p., accessed June 27, 2017, at https://pubs.usgs.gov/tm/tm6a39/.

Markstrom, S.L., Niswonger, R.G., Regan, R.S., Prudic, D.E., and Barlow, P.M., 2008, GSFLOW—Coupled Ground-water and Surface-water FLOW model based on the integration of the Precipitation-Runoff Modeling System (PRMS) and the Modular Ground-Water Flow Model (MODFLOW-2005): U.S. Geological Survey Techniques and Methods, book 6, chap. D1, 240 p., accessed June 27, 2017, at https://pubs.usgs.gov/ $\mathrm{tm} / \mathrm{tm} 6 \mathrm{~d} 1 /$.

Markstrom, S.L., Regan, R.S., Hay, L.E., Viger, R.J., Webb, R.M., Payn, R.A., and LaFontaine, J.H., 2015, PRMS-IV, the Precipitation-Runoff Modeling System, version 4: U.S. Geological Survey Techniques and Methods, book 6, chap. B7, 158 p., accessed June 27, 2017, at https://dx.doi.org/10.3133/tm6B7.

McDonald, M.G., and Harbaugh, A.W., 1984, A modular three-dimensional finite-difference ground-water flow model: U.S. Geological Survey Open-File Report 83-875, 528 p., accessed June 27, 2017, at https: //pubs.er.usgs.gov/publication/ofr83875.

McDonald, M.G., and Harbaugh, A.W., 1988, A modular three-dimensional finite-difference ground-water flow model: U.S. Geological Survey Techniques of Water-Resources Investigations, book 6, chap. A1, 586 p., accessed June 27, 2017, at https://pubs.er.usgs.gov/publication/twri06A1.

McDonald, M.G., Harbaugh, A.W., Orr, B.R., and Ackerman, D.J., 1992, A method of converting no-flow cells to variable-head cells for the U.S. Geological Survey modular finite-difference ground-water flow model: U.S. Geological Survey Open-File Report 91-536, 99 p., accessed June 27, 2017, at https://pubs. er.usgs.gov/publication/ofr91536.

Mehl, S.W., and Hill, M.C., 2013, MODFLOW-LGR_-Documentation of ghost node Local Grid Refinement (LGR2) for multiple areas and the Boundary Flow and Head (BFH2) Package: U.S. Geological Survey Techniques and Methods, book 6, chap. A44, 43 p., accessed June 27, 2017, at https://pubs.usgs.gov/tm/ $6 \mathrm{a} 44 \%$.

Merritt, M.L., and Konikow, L.F., 2000, Documentation of a computer program to simulate lake-aquifer interaction using the MODFLOW ground-water flow model and the MOC3D solute-transport model: U.S. Geological Survey Water-Resources Investigations Report 00-4167, 146 p., accessed June 27, 2017, at https://pubs.er.usgs.gov/publication/wri004167.

Moridis, G.J., and Pruess, Karsten, 1992, TOUGH simulations of Updegraff's set of fluid and heat flow problem: Ernest Orlando Lawrence Berkeley National Laboratory, LBNL Paper LBNL-32611, 223 p., accessed July 5, 2017, at http://escholarship.org/uc/item/9nt0r7wp.

Narasimhan, T.N., and Witherspoon, P.A., 1976, An integrated finite difference method for analyzing fluid flow in porous media: Water Resources Research, v. 12, no. 1, p. 57-64, accessed June 27, 2017, at https: //doi.org/10.1029/WR012i001p00057.

Niswonger, R.G., and Prudic, D.E., 2005, Documentation of the Streamflow-Routing (SFR2) Package to include unsaturated flow beneath streams-A modification to SFR1: U.S. Geological Survey Techniques 


\section{R-6 Documentation for the MODFLOW 6 Groundwater Flow Model}

and Methods, book 6, chap. A13, 50 p., accessed June 27, 2017, at https://pubs.er.usgs.gov/publication/ tm6A13.

Niswonger, R.G., Prudic, D.E., and Regan, R.S., 2006, Documentation of the Unsaturated-Zone Flow (UZF1) Package for modeling unsaturated flow between the land surface and the water table with MODFLOW2005: U.S. Geological Survey Techniques and Methods, book 6, chap. A19, 62 p., accessed June 27, 2017, at https://pubs.usgs.gov/tm/2006/tm6a19/.

Niswonger, R.G., Panday, Sorab, and Ibaraki, Motomu, 2011, MODFLOW-NWT, A Newton formulation for MODFLOW-2005: U.S. Geological Survey Techniques and Methods, book 6, chap. A37, 44 p., accessed June 27, 2017, at https://pubs.er.usgs.gov/publication/tm6A37.

Painter, Scott, Başağaoğlu, Hakan, and Liu, Angang, 2008, Robust representation of dry cells in single-layer MODFLOW models: Ground Water, v. 46, no. 6, p. 873-881, accessed June 27, 2017, at https://doi.org/10. 1111/j.1745-6584.2008.00483.x.

Panday, Sorab, Langevin, C.D., Niswonger, R.G., Ibaraki, Motomu, and Hughes, J.D., 2013, MODFLOWUSG version 1-An unstructured grid version of MODFLOW for simulating groundwater flow and tightly coupled processes using a control volume finite-difference formulation: U.S. Geological Survey Techniques and Methods, book 6, chap. A45, 66 p., accessed June 27, 2017, at https://pubs.usgs.gov/tm/06/a45/.

Peaceman, D.W., 1977, Fundamentals of numerical reservoir simulation: New York, Elsevier Scientific Publishing, $176 \mathrm{p}$.

Peaceman, D.W., 1983, Interpretation of well-block pressures in numerical reservoir simulation with nonsquare grid blocks and anisotropic permeability: Society of Petroleum Engineers Journal, v. 23, no. 03, p. 531-543, accessed June 27, 2017, at https://doi.org/10.2118/10528-PA.

Prudic, D.E., 1989, Documentation of a computer program to simulate stream-aquifer relations using a modular, finite-difference, ground-water flow model: U.S. Geological Survey Open-File Report 88-729, 113 p., accessed June 27, 2017, at https://pubs.er.usgs.gov/publication/ofr88729.

Prudic, D.E., Konikow, L.F., and Banta, E.R., 2004, A New Streamflow-Routing (SFR1) Package to simulate stream-aquifer interaction with MODFLOW-2000: U.S. Geological Survey Open File Report 2004-1042, 104 p., accessed June 27, 2017, at https://pubs.er.usgs.gov/publication/ofr20041042.

Remson, Irwin, Hornberger, G.M., and Molz, F.J., 1971, Numerical methods in subsurface hydrology: New York, Wiley-Interscience, $389 \mathrm{p}$.

Rorabaugh, M.I., 1953, Graphical and theoretical analysis of step-drawdown test of artesian well: Proceedings of the American Society of Civil Engineers, v. 79, no. 362, p. 1-23.

Rushton, K.R., and Redshaw, S.C., 1979, Seepage and groundwater flow-Numerical analysis by analogue and digital methods: New York, John Wiley and Sons, 339 p.

Saad, Yousef, 2003, Iterative methods for sparse linear systems: Philadelphia, Penn., Society for Industrial and Applied Mathematics, 528 p., accessed June 27, 2017, at https://doi.org/10.1137/1.9780898718003.

Schmid, Wolfgang, Hanson, R.T., Maddock, Thomas, III, and Leake, S.A., 2006, User guide for the farm process (FMP1) for the U.S. Geological Survey's modular three-dimensional finite-difference ground-water flow model, MODFLOW-2000: U.S. Geological Survey Techniques and Methods, book 6, chap. A17, 127 p., accessed June 27, 2017, at https://pubs.usgs.gov/tm/2006/tm6A17/.

Shoemaker, W.B., Kuniansky, E.L., Birk, Steffen, Bauer, Sebastian, and Swain, E.D., 2008, Documentation of a conduit flow process (CFP) for MODFLOW-2005: U.S. Geological Survey Techniques and Methods, book 6, chap. A24, 50 p., accessed June 27, 2017, at https://pubs.er.usgs.gov/publication/tm6A24.

Smith, R.E., 1983, Approximate sediment water movement by kinematic characteristics: Soil Science Society of America Journal, v. 47, p. 3-8, accessed June 27, 2017, at https://doi.org/10.2136/sssaj1983. $03615995004700010001 x$. 
Thiem, Günther, 1906, Hydrologische Methoden: Leipzig, Germany, J.M. Gebhardt, 56 p.

Trescott, P.C., 1976, Documentation of finite-difference model for simulation of three-dimensional groundwater flow: U.S. Geological Survey Open-File Report 76-591, 21 p., accessed June 27, 2017, at https: //pubs.er.usgs.gov/publication/ofr76591.

Trescott, P.C., and Larson, S.P., 1976, Supplement to Open-File Report 75-438, Documentation of finitedifference model for simulation of three-dimensional ground-water flow: U.S. Geological Survey Open-File Report 76-591, 21 p.

Trescott, P.C., Pinder, G.F., and Larson, S.P., 1976, Finite-difference model for aquifer simulation in two dimensions with results of numerical experiments: U.S. Geological Survey Techniques of Water-Resources Investigations, book 7, chap. C1, 116 p., accessed June 27, 2017, at https://pubs.usgs.gov/twri/twri7-c1/.

Voss, C.I., and Provost, A.M., 2010, SUTRA - a model for saturated-unsaturated, variable-density groundwater flow with solute or energy transport: U.S. Geological Survey Water-Resources Investigations Report 02-4231, $291 \mathrm{p}$.

Wang, H.F., and Anderson, M.P., 1982, Introduction to groundwater modeling-Finite difference and finite element methods: New York, W.H. Freeman and Company, 237 p.

Zheng, Chunmiao, and Wang, P.P., 1999, MT3DMS-A modular three-dimensional multi-species transport model for simulation of advection, dispersion and chemical reactions of contaminants in groundwater systems; Documentation and user's guide: Contract report SERDP-99-1: Vicksburg, Miss., U.S. Army Engineer Research and Development Center, $169 \mathrm{p}$.

Zheng, Chunmiao, Hill, M.C., and Hsieh, P.A., 2001, MODFLOW-2000, the U.S. Geological Survey Modular Ground-Water Model-User guide to the LMT6 package, the linkage with MT3DMS for multi-species mass transport modeling: U.S. Geological Survey Open-File Report 01-82, 43 p., accessed June 27, 2017, at https://pubs.er.usgs.gov/publication/ofr0182. 


\section{Appendix A. List of Symbols}

The following is a list of symbols that are used in this report.

Table A-1. List of symbols used in this report.

\begin{tabular}{|c|c|c|c|}
\hline Symbol & Description & Dimension & Definition \\
\hline$A$ & cross-sectional area perpendicular to flow & $L^{2}$ & eq. $4-13$ \\
\hline A & a matrix of the coefficients of head & $L^{2} / T$ & eq. $2-24$ \\
\hline$A_{l}$ & surface area of lake $l$ & $L^{2}$ & eq. $7-16$ \\
\hline$\alpha$ & $\begin{array}{l}\text { factor to convert the provider flow rate }\left(Q_{R}\right) \text { to the receiver flow rate } \\
\qquad\left(Q_{R}\right) \text { in the MVR Package }\end{array}$ & - & page $7-47$ \\
\hline$\alpha_{j}$ & contributing fraction for each contributing cell, $j$ & - & eq. $4-60$ \\
\hline$\alpha_{n}$ & contributing fraction of cell $n$ & - & eq. 4-60 \\
\hline$A_{n}$ & horizontal area of cell $n$ & $L^{2}$ & eq. $4-1$ \\
\hline$A_{n b}$ & wetted area of lake in cell, perpendicular to flow, for boundary $n b$ & $L^{2}$ & eq. $7-15$ \\
\hline$A N G L D E G X$ & angle (in degrees) the connection makes with the $\mathrm{x}$-axis & - & table $3-1$ \\
\hline$a_{n, i s r c}$ & flow from external source $i s r c$ into cell $n$ & $L^{3} / T$ & eq. 2-6 \\
\hline$A_{n, m}$ & cross-sectional area between cells $n$ and $m$ & $L^{2}$ & eq. $4-42$ \\
\hline$A_{\Omega}$ & $\frac{1}{1-\Omega}$ & $1 / L$ & eq. $4-5$ \\
\hline$A R E A$ & cross-sectional area of a cell connection for cell $n$ & $L^{2}$ & page 3-6 \\
\hline$A_{S_{n b}}$ & cross-sectional wetted area of SFR boundary $n b$ & $L^{2}$ & eq. $7-7$ \\
\hline b & a vector of constant terms $(R H S)$ & $L^{3} / T$ & eq. $2-24$ \\
\hline$\beta$ & relative height of the water above $D_{e x t_{n b}}$ for UZF boundary $n b$ & - & eq. $7-87$ \\
\hline$b_{i}$ & $\begin{array}{l}\text { the difference between the water level in the well and the pump top } \\
\text { elevation }\end{array}$ & $L$ & eq. $7-53$ \\
\hline$B L A K_{n b}$ & thickness of lakebed sediments for lake boundary $n b$ & $L$ & eq. $7-17$ \\
\hline$b_{n}$ & saturated aquifer thickness in GWF node $n$ & $L$ & eq. $7-38$ \\
\hline BOT & bottom elevation of cell $n$ & $L$ & page 3-6 \\
\hline BOTM & cell elevations & $L$ & page $3-2$ \\
\hline$B O T_{n b}$ & bottom of the lake-aquifer connection cell & $L$ & eq. $7-16$ \\
\hline$b_{w}^{0}$ & $\begin{array}{l}\text { total screen length in GWF node } n\left(b_{w}^{0}=T O P_{n}-B O T_{n} \text { for a fully }\right. \\
\text { penetrating well })\end{array}$ & $L$ & eq. $7-41$ \\
\hline$C$ & conductance & $L^{2} / T$ & eq. $4-14$ \\
\hline$c$ & smoothing interval above $D_{e x t_{n b}}$, which is equal to $0.15 D_{e x t_{n b}}$ & $L$ & eq. $7-89$ \\
\hline$C_{M A W, n}^{0}$ & saturated cumulative well-aquifer conductance & $L^{2} / T$ & eq. $7-44$ \\
\hline$C_{n, m}^{0}$ & saturated horizontal conductance between cells $n$ and $m$ & $L^{2} / T$ & page $4-10$ \\
\hline$C_{\text {Barrier }}$ & horizontal flow barrier conductance & $L^{2} / T$ & page $4-20$ \\
\hline$C_{d_{n o}}$ & outlet discharge coefficient & - & eq. $7-21$ \\
\hline$C D R N_{n b}$ & conductance for drain boundary condition $n b$ & $L^{2} / T$ & eq. $6-29$ \\
\hline cellid & cell index for DIS, DISV, and DISU discretizations & - & page $3-1$ \\
\hline$C F W_{i}^{0}$ & full flowing well conductance for well $i$ & $L^{2} / T$ & eq. $7-54$ \\
\hline$C F W_{i}$ & flowing well conductance & $L^{2} / T$ & eq. $7-51$ \\
\hline$C G H B_{n b}$ & conductance for general head boundary condition $n b$ & $L^{2} / T$ & eq. $6-21$ \\
\hline$c_{i}$ & conductance of subprism $i$ & $L^{2} / T$ & eq. 4-19 \\
\hline CL12 & distance from the center of the cell $n$ to the face that it shares with $m$ & - & table $3-1$ \\
\hline$C L A K_{n b}$ & conductance of the lake-GWF cell connection for lake boundary $n b$ & $L^{2} / T$ & eq. $7-18$ \\
\hline
\end{tabular}




\section{A-2 Documentation for the MODFLOW 6 Groundwater Flow Model}

Table A-1. List of symbols used in this report.-Continued

\begin{tabular}{|c|c|c|c|}
\hline Symbol & Description & Dimension & Definition \\
\hline$C_{M A W, n}$ & cumulative well-aquifer conductance & $L^{2} / T$ & eq. $7-35$ \\
\hline$C_{n b}$ & conductance of boundary condition $n b$, which is connected to cell $\eta_{n}$ & $L^{2} / T$ & eq. $6-1$ \\
\hline$C_{n b}^{+}$ & perturbed conductance for boundary condition $n b$ at $h+\epsilon$ & $L^{2} / T$ & eq. $6-10$ \\
\hline$C_{n, m}$ & conductance between nodes $n$ and $m$ & $L^{2} / T$ & eq. $2-4$ \\
\hline$C_{n, m}^{\text {original }}$ & original horizontal conductance between cells $n$ and $m$ & $L^{2} / T$ & page $4-20$ \\
\hline$C R I V_{n, 2}$ & a conductance controlling flow from the river into cell $n$ & $L^{2} / T$ & eq. $2-8$ \\
\hline$C R I V_{n b}$ & conductance for river boundary condition $n b$ & $L^{2} / T$ & eq. $6-22$ \\
\hline$C S F R_{n b}$ & streambed conductance for SFR boundary $n b$ & $L^{2} / T$ & eq. $7-12$ \\
\hline$C_{u}$ & $\begin{array}{l}\text { units constant, which is } 1.0 \text { for units of } \mathrm{m}^{3} \mathrm{sec}^{-1} \text { or } 1.486 \text { for units of } \\
\mathrm{ft}^{3} \mathrm{sec}^{-1}\end{array}$ & - & eq. $7-7$ \\
\hline$d$ & user-specified surface depth (SURFDEP shown in fig. 7-17) & $L$ & eq. 7-92 \\
\hline$D(\theta)$ & hydraulic diffusivity & $L^{2} / T$ & eq. $7-76$ \\
\hline$D E L C$ & width of a row & $L$ & page $3-2$ \\
\hline$D E L R$ & width of a column & $L$ & page $3-2$ \\
\hline$\Delta$ & difference operator & - & eq. $2-3$ \\
\hline$\Delta h_{n}$ & change in head in cell $n$ & $L$ & eq. $2-15$ \\
\hline$\Delta \mathbf{h}^{t, k}$ & head upgrade vector & $L$ & eq. $2-25$ \\
\hline$\Delta Q_{n, m}^{G N C}$ & ghost node correction term & $L^{3} / T$ & eq. $4-64$ \\
\hline$\Delta \theta$ & $\begin{array}{l}\text { positive change in water content between point } p \text { and a location a small } \\
\text { distance below point } p \text { along a trailing wave }\end{array}$ & $L^{3} / L^{3}$ & eq. $7-84$ \\
\hline$\Delta v_{\text {Barrier }}$ & $\begin{array}{l}\text { vertical thickness of the horizontal barrier (average saturated thickness } \\
\text { of the two cells) }\end{array}$ & $L$ & eq. $4-58$ \\
\hline$\Delta v_{n}$ & saturated thickness of cell $n$ & $L$ & eq. 4-1 \\
\hline$\Delta v_{n, m}$ & height of the face through which flow occurs & $L$ & eq. $2-3$ \\
\hline$\Delta w_{n, m}$ & width of the face through which flow occurs & $L$ & eq. $2-3$ \\
\hline$D_{e x t}$ & unsaturated zone evapotranspiration extinction depth & $L$ & page $7-39$ \\
\hline$D_{n b}$ & stream depth at the midpoint of reach $n b$ & $L$ & eq. $7-8$ \\
\hline$D_{n o}$ & water depth above the outlet invert elevation for outlet no & $L$ & eq. $7-19$ \\
\hline$\epsilon$ & head perturbation value & $L$ & eq. $6-10$ \\
\hline$\eta$ & height above land surface relative to $d$ & - & eq. $7-93$ \\
\hline$\eta_{n}$ & list of the cells connected to cell $n$ & - & eq. $2-14$ \\
\hline$E V T R_{n b}$ & maximum evapotranspiration rate for evapotranspiration boundary $n b$ & $L / T$ & eq. $6-30$ \\
\hline$E X D P_{n b}$ & cutoff or extinction depth for evapotranspiration boundary $n b$ & $L$ & eq. $6-30$ \\
\hline$f_{n b}$ & smoothing function that varies from 0 to 1 over a defined head interval & - & eq. $6-2$ \\
\hline$g$ & gravitational acceleration constant & $L / T^{2}$ & eq. $7-21$ \\
\hline$h$ & potentiometric head & $L$ & eq. $2-1$ \\
\hline $\mathbf{h}$ & a vector of head values & $L$ & eq. $2-24$ \\
\hline$h_{\bar{n}}$ & ghost node head & $L$ & eq. $4-60$ \\
\hline$H B_{n b}$ & specified head of boundary condition $n b$, which is connected to cell $\eta_{n}$ & $L$ & eq. $6-1$ \\
\hline$H C O F_{n}$ & value of head coefficient for cell $n$ & $L^{2} / T$ & eq. $2-22$ \\
\hline$H D R N_{n b}$ & elevation assigned to drain boundary condition $n b$ & $L$ & eq. $6-29$ \\
\hline$H G H B_{n b}$ & head assigned to general head boundary condition $n b$ & $L$ & eq. $6-21$ \\
\hline$H L A K_{l}$ & simulated stage in lake $l$ & $L$ & eq. $7-15$ \\
\hline$h_{m}$ & head at node $m$ & $L$ & eq. $2-3$ \\
\hline
\end{tabular}


Table A-1. List of symbols used in this report.-Continued

\begin{tabular}{|c|c|c|c|}
\hline Symbol & Description & Dimension & Definition \\
\hline$H M A W$ & head in a multi-aquifer well & $L$ & eq. $7-33$ \\
\hline$H M A W_{i}^{t_{\text {old }}}$ & head in MAW well $i$ at the end of the previous time step & $L$ & eq. $7-54$ \\
\hline$h_{n}$ & head at node $n$ & $L$ & eq. $2-3$ \\
\hline$H O L D$ & head at the end of the previous time step $\left(h^{t_{\text {old }}}\right)$ & $L$ & eq. $2-18$ \\
\hline$H R I V_{n b}$ & stage assigned to river boundary condition $n b$ & $L$ & eq. $6-22$ \\
\hline$h_{\text {root }_{n b}}$ & user-specified negative root pressure for UZF boundary $n b$ & $L$ & eq. $7-85$ \\
\hline$H S F R_{n b}$ & stage assigned to SFR boundary $n b$ & $L$ & eq. $7-1$ \\
\hline$H W V A$ & $\begin{array}{l}\text { width perpendicular to flow for horizontal connection and face area for } \\
\text { a vertical connection }\end{array}$ & $L$ or $L^{2}$ & table $3-1$ \\
\hline$I A C$ & starting position of $\mathbf{A}$ entries for a row & - & table $3-1$ \\
\hline ICELLTYPE & integer variable that defines if a cell is convertible or confined & - & page 4-1 \\
\hline IDOMAIN & variable used to exclude cells from the model & - & page $3-5$ \\
\hline$i_{E T}$ & unsaturated zone evapotranspiration rate per unit depth & $L / T / L$ & eq. $7-76$ \\
\hline$I H C$ & horizontal connection code for each $n-m$ connection & - & table $3-1$ \\
\hline$I_{n b}$ & recharge flux for boundary condition $n b$ & $L / T$ & eq. $6-20$ \\
\hline $\mathbf{J}$ & Jacobian matrix & $L^{3} / T / L$ & eq. $2-25$ \\
\hline$J A$ & column indices for $\mathbf{A}$ entries & - & table $3-1$ \\
\hline$K$ & hydraulic conductivity & $L / T$ & eq. $4-13$ \\
\hline K & hydraulic-conductivity tensor & $L / T$ & eq. $2-1$ \\
\hline$K(\theta)$ & $\begin{array}{l}\text { vertical unsaturated hydraulic conductivity as a function of water con- } \\
\text { tent }\end{array}$ & $L / T$ & eq. $7-76$ \\
\hline$K_{11}$ & $\begin{array}{l}\text { user-specified value of hydraulic conductivity for the first principal } \\
\text { direction }\end{array}$ & $L / T$ & page $4-16$ \\
\hline$K_{11_{n}}$ & hydraulic conductivity of principal axis for GWF node $n$ & $L / T$ & eq. $7-38$ \\
\hline$K_{22}$ & value of hydraulic conductivity for the second principal direction & $L / T$ & page $4-16$ \\
\hline$K_{22_{n}}$ & hydraulic conductivity of secondary axis for GWF node $n$ & $L / T$ & eq. $7-38$ \\
\hline$K_{33}$ & $\begin{array}{l}\text { user-specified value of hydraulic conductivity for the third principal } \\
\text { direction }\end{array}$ & $L / T$ & page $4-16$ \\
\hline$K_{\text {Barrier }}$ & hydraulic conductivity of the horizontal flow barrier & $L / T$ & eq. $4-58$ \\
\hline$K L A K_{n b}$ & $\begin{array}{l}\text { hydraulic conductivity of the lakebed sediments underlying the lake for } \\
\text { lake boundary } n b\end{array}$ & $L / T$ & eq. $7-17$ \\
\hline$K_{n b}$ & hydraulic conductivity of the material for boundary $n b$ & $L / T$ & page $6-16$ \\
\hline$K_{n, m}$ & hydraulic conductivity of cell $n$ in the direction of cell $m$ & $L / T$ & eq. 4-27 \\
\hline $\bar{K}_{n, m}$ & effective hydraulic conductivity between the nodes $n$ and $m$ & $L / T$ & eq. $2-3$ \\
\hline$K_{s}$ & hydraulic conductivity of the filter pack or damaged formation & $L / T$ & eq. $7-41$ \\
\hline$K_{\text {sat }}$ & saturated hydraulic conductivity & $L / T$ & eq. $7-83$ \\
\hline$k$ & iteration counter & - & page $2-20$ \\
\hline$K_{v_{n}}$ & vertical hydraulic conductivity of GWF cell $n$ & $L / T$ & eq. 7-91 \\
\hline$K_{x x}$ & hydraulic conductivity along the $x$ coordinate axis & $L / T$ & eq. $2-1$ \\
\hline$K_{y y}$ & hydraulic conductivity along the $y$ coordinate axis & $L / T$ & eq. $2-1$ \\
\hline$K_{z z}$ & hydraulic conductivity along the $z$ coordinate axis & $L / T$ & eq. $2-1$ \\
\hline$L_{1,2}$ & length of the prism parallel to the flow path between $h_{1}$ and $h_{2}$ & $L$ & eq. $4-13$ \\
\hline$L_{\text {Barrier }}$ & distance across the barrier in the flow direction & $L$ & eq. $4-58$ \\
\hline$L B O T_{n b}$ & lake bottom elevation for lake boundary $n b$ & $L$ & page $7-12$ \\
\hline
\end{tabular}




\section{A-4 Documentation for the MODFLOW 6 Groundwater Flow Model}

Table A-1. List of symbols used in this report.-Continued

\begin{tabular}{|c|c|c|c|}
\hline Symbol & Description & Dimension & Definition \\
\hline$L C 1^{0}$ & fully saturated aquifer-loss coefficient & $T / L^{2}$ & eq. $7-39$ \\
\hline$L C 1_{n}$ & linear aquifer-loss coefficient & $T / L^{2}$ & eq. $7-33$ \\
\hline$L C 2^{\circ}$ & fully saturated linear well-loss coefficient & $T / L^{2}$ & eq. $7-42$ \\
\hline$L C 2_{n}$ & linear well-loss coefficient & $T / L^{2}$ & eq. $7-33$ \\
\hline$L C 3$ & nonlinear well-loss coefficient & $T^{P} / L^{2 P}$ & eq. $7-33$ \\
\hline$L L A K_{n b}$ & lakebed leakance of lake boundary $n b$ & $1 / T$ & eq. $7-17$ \\
\hline$L_{m, n}$ & distance between node $m$ and the shared face with node $n$ & $L$ & eq. 4-24 \\
\hline$L_{n b}$ & length of boundary $n b$ within a cell & $L$ & page $6-16$ \\
\hline$L_{n b, n}$ & $\begin{array}{l}\text { distance from the lake bottom }\left(L B O T_{n b}\right) \text { to the center of the connected } \\
\text { cell }\end{array}$ & $L$ & eq. $7-15$ \\
\hline$L_{n, m}$ & distance between node $n$ and the shared face with node $m$ & $L$ & eq. $4-24$ \\
\hline$M A W_{i}$ & the GWF connections for MAW well $i$ & - & eq. $7-54$ \\
\hline$M_{n b}$ & area multiplier boundary condition $n b$ that is used to scale $I_{n b}$ & - & eq. $6-20$ \\
\hline$n$ & cell number used for DISV and DISU discretizations & - & page $3-1$ \\
\hline$\nabla h$ & head-gradient vector & $L / L$ & eq. $2-1$ \\
\hline$n b$ & boundary condition counter & - & eq. $6-1$ \\
\hline$N C O L$ & number of columns in a DIS discretization & - & page $3-2$ \\
\hline $\mathbf{n}_{g}$ & $\begin{array}{l}\text { unit vector in the direction normal to the interface expressed in coordi- } \\
\text { nates global model coordinates }\end{array}$ & - & page $4-18$ \\
\hline$N J A$ & $\begin{array}{l}\text { sum of the number of unique connections between cells and the number } \\
\text { of } N O D E S \text { (number of nonzero entries in } \mathbf{A} \text { ) }\end{array}$ & - & table $3-1$ \\
\hline $\mathbf{n}_{K}$ & $\begin{array}{l}\text { unit vector in the direction normal to the } n, m \text { interface expressed in } \\
\text { coordinates aligned with } K_{11}, K_{22} \text {, and } K_{33}\end{array}$ & - & page $4-18$ \\
\hline$n_{K 1}$ & $\begin{array}{l}x \text { component of the unit vector in the direction normal to the } n, m \\
\text { interface expressed in coordinates aligned with } K_{11} \text { and } K_{22}\end{array}$ & - & eq. $4-50$ \\
\hline$n_{K 2}$ & $\begin{array}{l}y \text { component of the unit vector in the direction normal to the } n, m \\
\text { interface expressed in coordinates aligned with } K_{11} \text { and } K_{22}\end{array}$ & - & eq. $4-50$ \\
\hline$n_{K 3}$ & $\begin{array}{l}z \text { component of the unit vector in the direction normal to the } n, m \\
\text { interface expressed in coordinates aligned with } K_{11}, K_{22} \text {, and } K_{33}\end{array}$ & - & eq. $4-51$ \\
\hline$N L A Y$ & number of layers in a DIS and DISV discretizations & - & page $3-2$ \\
\hline$n_{n b}$ & Manning's roughness coefficient for SFR boundary $n b$ & $T / L^{1 / 3}$ & eq. $7-7$ \\
\hline$n_{n o}$ & Manning's roughness coefficient for outlet no & $T / L^{1 / 3}$ & eq. $7-19$ \\
\hline NODES & number of nodes (rows) in the model & - & table $3-1$ \\
\hline$N R O W$ & number of rows in a DIS discretization & - & page $3-2$ \\
\hline NVERT & number of vertices for the cell & - & page $3-4$ \\
\hline$\Omega$ & smoothing distance & $L$ & eq. $4-5$ \\
\hline$\partial$ & partial differential operator & - & eq. $2-2$ \\
\hline$P E T$ & potential evapotranspiration & $L / T$ & page $7-39$ \\
\hline $\operatorname{PETM}_{n b_{n s-1}}$ & $\begin{array}{l}\text { proportion (between zero and one) of the maximum evapotranspiration } \\
\quad \text { rate }\left(Q E T M_{n b}\right) \text { for segment } n s\end{array}$ & - & eq. $6-33$ \\
\hline$\Phi$ & $\begin{array}{l}\text { WEL Package scaling factor that ranges between } 0 \text { and } 1 \text { and typically } \\
\text { is a small value }\end{array}$ & - & eq. $6-17$ \\
\hline$P_{n}$ & sum source and sink head coefficient terms & $L^{2} / T$ & eq. $2-11$ \\
\hline$p_{n, i s r c}$ & source term conductance & $L^{2} / T$ & eq. $2-6$ \\
\hline
\end{tabular}


Table A-1. List of symbols used in this report.-Continued

\begin{tabular}{|c|c|c|c|}
\hline Symbol & Description & Dimension & Definition \\
\hline$\psi(\theta)$ & sediment capillary pressure in UZF boundary at point $p$ & $L$ & eq. $7-85$ \\
\hline$P$ & power (exponent) of the nonlinear discharge component of well loss & - & eq. $7-33$ \\
\hline$P X D P_{n b_{n s-1}}$ & $\begin{array}{l}\text { proportion (between zero and one) of the extinction depth }\left(E X D P_{n b}\right) \\
\quad \text { for segment } n s\end{array}$ & - & eq. $6-33$ \\
\hline$Q$ & volumetric flow & $L^{3} / T$ & eq. $4-13$ \\
\hline $\mathbf{q}$ & vector of specific discharge & $L / T$ & eq. $2-1$ \\
\hline$Q_{s}^{\prime}$ & volumetric flux per unit volume representing sources and sinks of water & $1 / T$ & eq. $2-2$ \\
\hline$q_{a}$ & volumetric infiltration rate per unit area & $L^{3} / L^{2} T$ & eq. $7-90$ \\
\hline$q_{c}$ & fluid flux along a connection & $L / T$ & eq. $4-47$ \\
\hline$Q D R N_{n b}$ & flow into cell $n$ from the drain boundary $n b$ & $L^{3} / T$ & eq. $6-29$ \\
\hline$Q E T_{l}$ & evaporation from lake $l$ & $L^{3} / T$ & eq. $7-23$ \\
\hline$Q E T M_{n b}$ & maximum value of $Q E T_{n b}$ & $L^{3} / T$ & page $6-26$ \\
\hline$Q E T_{n b}$ & flow into cell $n$ from the evapotranspiration boundary $n b$ & $L^{3} / T$ & eq. $6-31$ \\
\hline$Q F W_{i}$ & flowing well discharge for multi-aquifer well $i$ & $L^{3} / T$ & eq. $7-51$ \\
\hline$Q G H B_{n b}$ & flow into cell $n$ from the general head boundary condition $n b$ & $L^{3} / T$ & eq. $6-21$ \\
\hline$q_{i}$ & flow across subprism $i$ & $L^{3} / T$ & eq. $4-19$ \\
\hline$Q I N F_{l}$ & specified inflow to lake $l$ & $L^{3} / T$ & eq. $7-22$ \\
\hline$Q I N_{l}$ & total inflow to lake $l$ & $L^{3} / T$ & eq. $7-22$ \\
\hline$Q L A K I_{n b}$ & groundwater leakage to a lake for lake boundary $n b$ & $L^{3} / T$ & eq. $7-22$ \\
\hline$Q L A K_{n b}$ & flow into cell $n$ from LAK boundary $n b$ & $L^{3} / T$ & eq. $7-15$ \\
\hline$Q L A K O_{n b}$ & groundwater leakage from a lake for lake boundary $n b$ & $L^{3} / T$ & eq. $7-23$ \\
\hline$Q M A W$ & flow between cell $n$ and the multi-aquifer well & $L^{3} / T$ & eq. $7-33$ \\
\hline$Q M A W_{i}^{+}$ & the specified flow for MAW well $i$ at $H M A W_{i}+\epsilon$ & $L$ & eq. $7-74$ \\
\hline$Q M A W_{i}$ & applied multi-aquifer well pumping rate & $L^{3} / T$ & eq. $7-52$ \\
\hline$Q M A W_{i}^{0}$ & user-specified multi-aquifer well pumping rate for well $i$ & $L^{3} / T$ & eq. $7-52$ \\
\hline$Q M A W S_{i}$ & flow resulting from multi-aquifer well storage changes & $L^{3} / T$ & eq. $7-64$ \\
\hline$Q \max _{n b}$ & maximum river leakage & $L^{3} / T$ & eq. $6-26$ \\
\hline$Q_{m d p t_{n b}}$ & flow at the midpoint of the reach for SFR boundary $n b$ & $L^{3} / T$ & eq. $7-6$ \\
\hline$Q M V R_{n b}$ & inflow from the Mover Package for lake $l$ & $L^{3} / T$ & eq. $7-22$ \\
\hline$Q_{n}$ & sum of source and sink flow terms & $L^{3} / T$ & eq. $2-12$ \\
\hline$Q_{n b}$ & $\begin{array}{l}\text { specified flow rate of boundary condition } n b \text {, which is connected to cell } \\
\quad \eta_{n}\end{array}$ & $L^{3} / T$ & eq. $6-1$ \\
\hline$Q_{n b}^{+}$ & perturbed specified flow for boundary condition $n b$ at $h+\epsilon$ & $L^{3} / T$ & eq. $6-13$ \\
\hline$Q_{n b}^{0}$ & user-specified flow rate for boundary condition $n b$ & $L^{3} / T$ & eq. $6-11$ \\
\hline$q_{n, i s r c}$ & specified source term & $L^{3} / T$ & eq. $2-6$ \\
\hline$Q_{n, m}$ & flow rate into cell $n$ from cell $m$ & $L^{3} / T$ & eq. $2-3$ \\
\hline$Q_{n, s}$ & flow rate of sources and sinks into cell $n$ & $L^{3} / T$ & eq. $2-14$ \\
\hline$Q O U T_{l}$ & total outflow to lake $l$ & $L^{3} / T$ & eq. $7-23$ \\
\hline QOUTLET & outlet discharge rate for outlet no & $L^{3} / T$ & eq. $7-19$ \\
\hline$Q_{P}$ & available rate of water from the provider in the MVR Package & $L^{3} / T$ & page $7-47$ \\
\hline$Q P P T_{n b}$ & precipitation that falls directly on lake $l$ & $L^{3} / T$ & eq. $7-22$ \\
\hline$Q_{R}$ & rate of water that is applied to the receiver by the MVR Package & $L^{3} / T$ & page $7-47$ \\
\hline$Q R I V_{n b}$ & flow into cell $n$ from the river boundary $n b$ & $L^{3} / T$ & eq. $6-22$ \\
\hline
\end{tabular}




\section{A-6 Documentation for the MODFLOW 6 Groundwater Flow Model}

Table A-1. List of symbols used in this report.-Continued

\begin{tabular}{|c|c|c|c|}
\hline Symbol & Description & Dimension & Definition \\
\hline$Q R_{n b}$ & recharge flow rate applied to boundary condition $n b$ & $L^{3} / T$ & eq. $6-20$ \\
\hline$Q R O_{l}$ & direct overland runoff to lake $l$ & $L^{3} / T$ & eq. $7-22$ \\
\hline$Q R O_{n b}$ & direct overland runoff to a reach for SFR boundary $n b$ & $L^{3} / T$ & eq. $7-3$ \\
\hline$Q_{S}$ & user-specified rate of water used to calculate $Q_{R}$ in the MVR Package & $L^{3} / T$ & page $7-47$ \\
\hline$Q S F R I_{n b}$ & groundwater leakage to a reach for SFR boundary $n b$ & $L^{3} / T$ & eq. $7-3$ \\
\hline$Q S F R_{n b}$ & flow into cell $n$ from SFR boundary $n b$ & $L^{3} / T$ & eq. $7-1$ \\
\hline$Q S R C_{n b}$ & sum of available sources and sinks for SFR boundary $n b$ & $L^{3} / T$ & eq. $7-11$ \\
\hline$\hat{Q} S R C_{n b}$ & available water in the lake & $L^{3} / T$ & eq. $7-29$ \\
\hline$Q S R I_{n b}$ & specified inflow for SFR boundary $n b$ & $L^{3} / T$ & eq. $7-3$ \\
\hline$Q_{S S_{n}}$ & volumetric flow rate from confined storage in cell $n$ & $L^{3} / T$ & eq. $5-4$ \\
\hline$Q_{S T O}$ & change in the volume of water stored in cell $n$ & $L^{3} / T$ & eq. $2-14$ \\
\hline$Q_{S y_{n}}$ & volumetric flow rate from unconfined storage in cell $n$ & $L^{3} / T$ & eq. $5-11$ \\
\hline$Q T R B_{n b}$ & sum of tributary flow from upstream reaches for SFR boundary $n b$ & $L^{3} / T$ & eq. $7-3$ \\
\hline$Q U G E T_{n b}$ & $\begin{array}{l}\text { volumetric rate of evapotranspiration removed from GWF cell } n \text { con- } \\
\text { nected to UZF boundary } n b\end{array}$ & $L^{3} / T$ & eq. $7-86$ \\
\hline$Q U G W S_{n b}$ & volumetric rate of groundwater seepage to land surface & $L^{3} / T$ & eq. 7-91 \\
\hline$q_{U I N F}$ & minimum of the user-specified infiltration rate $\left(q_{a}\right)$ and $K_{s a t}$ & $L / T$ & eq. 7-95 \\
\hline$Q U I N F_{n b}$ & $\begin{array}{l}\text { volumetric net infiltration rate for UZF boundary } n b \text {, which is con- } \\
\text { nected to GWF cell } n\end{array}$ & $L^{3} / T$ & eq. $7-95$ \\
\hline$q_{U Z E T_{p}}$ & unsaturated zone evapotranspiration for UZF boundary at point $p$ & $L^{3} / T$ & eq. $7-85$ \\
\hline$q_{U Z F}$ & the vertical water flux in the unsaturated zone & $L^{3} / L^{2} T$ & eq. $7-76$ \\
\hline$Q W E L_{i}^{0}$ & user-specified well pumping rate for well $n b$ & $L^{3} / T$ & eq. $6-16$ \\
\hline$Q W E L_{n}$ & recharge (negative for pumping) from cell $n$ to a particular well & $L^{3} / T$ & eq. $6-15$ \\
\hline$Q W E L_{n b}$ & total recharge (negative for pumping) for the well & $L^{3} / T$ & eq. $6-15$ \\
\hline$Q W I T H_{l}$ & specified withdrawals from lake $l$ & $L^{3} / T$ & eq. $7-23$ \\
\hline $\mathbf{R}$ & rotation matrix & - & eq. $4-52$ \\
\hline $\mathbf{r}$ & residual vector & $L^{3} / t$ & eq. $2-25$ \\
\hline$R B O T_{n b}$ & bottom elevation of the river & $L$ & eq. $6-24$ \\
\hline$R E T_{n b}$ & evapotranspiration rate for evapotranspiration boundary $n b$ & $L / T$ & eq. $6-30$ \\
\hline$R H S_{n}$ & value of right-hand side for cell $n$ & $L^{3} / T$ & eq. $2-23$ \\
\hline$r_{n}$ & residual for cell $n$ & $L^{3} / T$ & eq. $2-27$ \\
\hline$R_{n, 2}$ & river head & $L$ & eq. $2-8$ \\
\hline$R_{n b}$ & hydraulic radius of the stream for SFR boundary $n b$ & $L$ & eq. $7-7$ \\
\hline$r_{o_{n}}$ & effective (or equivalent) radius of GWF node $n$ & $L$ & eq. $7-36$ \\
\hline$R P E T$ & $\begin{array}{l}\text { residual potential evapotranspiration-difference between } P E T \text { and } \\
\qquad q_{U Z E T_{p}}\end{array}$ & $L / T$ & page $7-39$ \\
\hline$r_{q}$ & flow radius, which is the average of $r_{w}$ and $r_{s}$ & $L$ & eq. $7-48$ \\
\hline$r_{s}$ & well skin radius & $L$ & eq. $7-41$ \\
\hline$r_{w}$ & multi-aquifer well radius & $L$ & eq. $7-36$ \\
\hline$S_{F_{n b}}^{*}$ & saturation for lake boundary & - & eq. $7-16$ \\
\hline$S_{F n, m}^{*}$ & upstream saturated fraction calculated in cells $n$ and $m$ & - & eq. $4-33$ \\
\hline$S_{M A W, n}^{*}$ & $\begin{array}{l}\text { screen saturation in GWF cell } n \text {, which is equal to the GWF cell sat- } \\
\text { uration }\left(S_{F_{n}}^{*}\right) \text { for conductances calculated using the aquifer- and } \\
\text { well-loss coefficients }\end{array}$ & - & eq. $7-40$ \\
\hline
\end{tabular}


Table A-1. List of symbols used in this report.-Continued

\begin{tabular}{|c|c|c|c|}
\hline Symbol & Description & Dimension & Definition \\
\hline$S_{S F R, n b}^{*}$ & cubic saturation function & - & eq. $7-1$ \\
\hline$S_{0_{n b}}$ & slope of the outlet channel for outlet no & - & eq. $7-19$ \\
\hline$s_{b}$ & maximum bottom elevation of cell $n$ and cell $m$ & $L$ & eq. $4-11$ \\
\hline$S B O T_{n b}$ & $\begin{array}{l}\text { elevation of the bottom of the streambed sediments for SFR boundary } \\
n b\end{array}$ & $L$ & eq. $7-1$ \\
\hline$S C 1_{n}$ & "storage capacity" or the "primary storage capacity" of cell $n$ & $L^{2}$ & page $5-1$ \\
\hline$S C 2_{n}$ & "secondary storage capacity" of cell $n$ & $L^{2}$ & page $5-1$ \\
\hline$S C L L E N_{i}$ & $\begin{array}{l}\text { distance above the pump top elevation below which the user-specified } \\
\text { multi-aquifer pumping rate is reduced }\end{array}$ & $L$ & eq. $7-53$ \\
\hline$S_{F}$ & saturated cell fraction & - & eq. $4-6$ \\
\hline$S_{F}^{*}$ & smoothed saturated cell fraction & - & eq. 4-5 \\
\hline$S_{F W_{i}}$ & the fraction of the flowing well scaling length above the top of well $i$ & - & eq. $7-54$ \\
\hline$S K I N_{n}$ & $\begin{array}{l}\text { skin coefficient, which represents a zone of affected hydraulic properties } \\
\text { close to the wellbore or well screen }\end{array}$ & - & eq. $7-41$ \\
\hline$S_{Q M A W_{i}}$ & fraction of the pump rate scaling length above the pump top elevation & - & eq. $7-52$ \\
\hline$S S$ & specific storage of the porous material & $1 / L$ & eq. $2-2$ \\
\hline$S S_{n}$ & $\begin{array}{l}\text { volume of water that can be injected per unit volume of aquifer material } \\
\text { per unit change in head in cell } n\end{array}$ & $1 / L$ & eq. $2-15$ \\
\hline$s_{t}$ & minimum top elevation of cell $n$ and cell $m$ & $L$ & eq. $4-10$ \\
\hline$S_{U G E T, n}$ & $\begin{array}{l}\text { smoothing function that scales the remaining potential evapotranspira- } \\
\text { tion }\end{array}$ & - & eq. $7-86$ \\
\hline$S_{U G W S, n}$ & $\begin{array}{l}\text { smoothing function that scales the groundwater seepage to land surface } \\
\text { based on the height of water above land surface in cell } n\end{array}$ & - & eq. 7-91 \\
\hline$S_{U I N F, n}$ & $\begin{array}{l}\text { smoothing function that scales the user-specified infiltration based on } \\
\text { the position of the water table relative to land surface in cell } n\end{array}$ & - & eq. 7-95 \\
\hline$\sum_{j \in \eta_{n}}$ & summation over all $j$ contributing cells & - & eq. $4-60$ \\
\hline$\sum T$ & sum of the transmissivities of all nodes penetrated by well $n b$ & $L^{2} / T$ & eq. $6-15$ \\
\hline$S U R F_{n b}$ & evapotranspiration surface elevation for evapotranspiration boundary $n b$ & $L$ & eq. $6-30$ \\
\hline$S_{W E L_{n b}}$ & fraction of the specified pumping rate for the cell containing well $n b$ & - & eq. $6-16$ \\
\hline$S y_{n}$ & specific yield of cell $n$ & $L^{2}$ & page $5-3$ \\
\hline$t$ & time & $T$ & eq. $2-2$ \\
\hline$T$ & transmissivity & $L^{2} / T$ & eq. $4-16$ \\
\hline $\bar{T}$ & $\begin{array}{l}\text { average transmissivity between horizontally adjacent cells } n \text { and } m \text { with } \\
\text { linearly varying hydraulic properties }\end{array}$ & $L^{2} / T$ & eq. $4-28$ \\
\hline$T_{n}^{0}$ & fully saturated transmissivity of cell $n$ & $L^{2} / T$ & eq. $7-39$ \\
\hline$\theta$ & volumetric water content (volume of water per volume of rock) & $L^{3} / L^{3}$ & eq. $7-76$ \\
\hline$\theta_{1}$ & $\begin{array}{l}\text { counterclockwise rotation angle for the hydraulic conductinity ellipse } \\
\text { within the }(x, y) \text { plane (yaw) }\end{array}$ & $\circ$ & page $4-18$ \\
\hline$\theta_{2}$ & $\begin{array}{l}\text { rotation of the conductivity ellipsoid upward or downward from the } \\
\quad(x, y) \text { plane (pitch) }\end{array}$ & $\circ$ & page $4-18$ \\
\hline$\theta_{3}$ & $\begin{array}{l}\text { rotation of the conductivity ellipsoid about the axis corresponding to } \\
K_{11} \text { (roll) }\end{array}$ & $\circ$ & page $4-18$ \\
\hline$\theta_{\text {ext }}$ & unsaturated zone evapotranspiration extinction water content & $L^{3} / L^{3}$ & page $7-39$ \\
\hline$\theta_{p}$ & water content at point $p$ & $L^{3} / L^{3}$ & eq. $7-84$ \\
\hline$\theta_{q_{a}}$ & water content of a wave generated from infiltration & $L^{3} / L^{3}$ & eq. 7-90 \\
\hline
\end{tabular}




\section{A-8 Documentation for the MODFLOW 6 Groundwater Flow Model}

Table A-1. List of symbols used in this report.-Continued

\begin{tabular}{|c|c|c|c|}
\hline Symbol & Description & Dimension & Definition \\
\hline$\theta_{\text {resid }}$ & residual (irreducible) water content & $L^{3} / L^{3}$ & eq. 7-83 \\
\hline$\theta_{\text {sat }}$ & saturated water content & $L^{3} / L^{3}$ & eq. $7-83$ \\
\hline$\theta_{t}$ & previous water content along the wetting or drying profile at time $t$ & $L^{3} / L^{3}$ & eq. $7-82$ \\
\hline$\theta_{t+\Delta t}$ & current water content along the wetting or drying profile at time $t+\Delta t$ & $L^{3} / L^{3}$ & eq. $7-82$ \\
\hline$\theta_{t_{0}}$ & initial volumetric water content of a wave moving through sediment & $L^{3} / L^{3}$ & eq. $7-81$ \\
\hline$\theta_{t_{1}}$ & current volumetric water content of a wave moving through sediment & $L^{3} / L^{3}$ & eq. $7-81$ \\
\hline THRESH & $\begin{array}{l}\text { threshold value that is used to convert a dry cell to wet based upon the } \\
\text { head in an adjacent cell }\end{array}$ & $L$ & page $4-19$ \\
\hline$T_{m, n}$ & transmissivity in cell $m$ in the direction of cell $n$ & $L^{2} / T$ & eq. 4-24 \\
\hline$T_{n}$ & transmissivity of cell $n$ & $L^{2} / T$ & eq. $7-36$ \\
\hline$T_{n, m}$ & transmissivity in cell $n$ in the direction of cell $m$ & $L^{2} / T$ & eq. $4-24$ \\
\hline$t_{\text {old }}$ & previous time step & $T$ & eq. $2-18$ \\
\hline TOP & top elevation of cell $n$ & $L$ & page 3-6 \\
\hline$T_{O} P_{i}$ & top elevation of the flowing well & $L$ & eq. $7-51$ \\
\hline$T O P_{n b}$ & top of the lake-aquifer connection cell & $L$ & eq. $7-16$ \\
\hline$t$ & time-step counter & - & page $2-20$ \\
\hline$v(\theta)$ & wave velocity restricted to the downward (positive $z$ ) direction & $L / T$ & eq. $7-79$ \\
\hline$v_{1}$ & current wave velocity & $L / T$ & eq. $7-81$ \\
\hline$\varepsilon$ & Brooks-Corey exponent & - & eq. $7-83$ \\
\hline$V_{n}$ & volume of cell $n$ & $L^{3}$ & eq. $2-15$ \\
\hline$v_{p}$ & trailing wave velocity at point $p$ & $L / T$ & eq. $7-84$ \\
\hline$W$ & width of the prism & $L$ & eq. $4-16$ \\
\hline WETFCT & $\begin{array}{l}\text { user-specified constant factor used to rewet a dry cell when the thresh- } \\
\text { old value }(T H R E S H) \text { is exceeded }\end{array}$ & - & eq. $4-56$ \\
\hline$W_{n b}$ & width of boundary $n b$ & $L$ & page $6-16$ \\
\hline$w_{n b}$ & horizontal width of the lake-aquifer connection cell & $L$ & eq. $7-16$ \\
\hline$W_{n o}$ & width of the outlet for outlet no & $L$ & eq. $7-19$ \\
\hline$x$ & $\mathrm{x}$ coordinate direction & $L$ & page $2-1$ \\
\hline$x_{c}$ & distance along a connection & $L$ & eq. $4-47$ \\
\hline$\xi$ & user-specified elevation relative to the top of cell $n$ & $L$ & eq. 7-91 \\
\hline$y$ & y coordinate direction & $L$ & page $2-1$ \\
\hline$z$ & $\mathrm{z}$ coordinate direction & $L$ & page $2-1$ \\
\hline$\zeta_{n}$ & $\Phi\left(T O P_{n}-B_{n} O T_{n}\right)$ & $L$ & eq. $6-17$ \\
\hline$z_{i}$ & pump top elevation & $L$ & eq. $7-53$ \\
\hline$z_{n b}$ & depth below which smoothing occurs & $L$ & eq. $7-88$ \\
\hline$z_{n o}$ & outlet invert elevation & $L$ & eq. $7-20$ \\
\hline
\end{tabular}




\section{Appendix B. Implementation of the Groundwater Flow Model in the MOD- FLOW 6 Framework}

The GWF Model is implemented in the MODFLOW 6 framework (Hughes and others, 2017) as a specific type of Numerical Model. Within the MODFLOW 6 object-oriented framework, any number of GWF Models can be represented in a simulation. Pointers to each of these model objects are stored in a list, which makes it possible to loop through this array of models and perform a task on each one. This appendix describes the design of the GWF Model, its primary procedures, and other aspects of the model. This appendix discussion focuses on a single groundwater model instance; however, the concepts are easily extended to simulations with multiple GWF Models. Hughes and others (2017) provide additional information about the MODFLOW 6 framework.

\section{Groundwater Flow Model Procedures}

The GWF Model is defined by the GwfModelType class, which is a subclass of NumericalModelType. A flowchart of the main program, selected Iterative Model Solution (IMS) procedures, and primary procedures of the GWF Model that are called from either an IMS procedure or from the main program are shown in figure B-1. As shown in this figure, GWF Model procedures are called both from the main program and from IMS procedures. This design pattern, which includes calls to the GWF Model procedures from the main program and from the IMS, facilitates solution of multiple models within a single set of matrix equations.

As shown in figure $\mathrm{B}-1$, the time-step loop is controlled by the main program. There is no longer a separate stress period loop in the main program, as there were in previous MODFLOW versions; however, the concept of a stress period remains, and period and time-step numbers are incremented accordingly. The following is a description of the procedures of the GWF Model that are called from the main program or from the IMS:

- At the start of the simulation, a Create (CR) Procedure is used to instantiate, or create an instance of, the GWF Model. The CR Procedure generates a new GWF Model object with the specified or default name, and a pointer to the model object is stored within the list of models. The CR Procedure also instantiates all of the packages associated with the GWF Model and assigns values for some of the data that is stored within the model.

- The Define (DF) Procedure opens files, reads information about the size of the model and how cells are connected, and creates and defines boundary packages.

- The Add Connections (AC) Procedure informs the IMS of the total number of GWF Model cells and how cells are connected to one another; this information is used by the IMS to set up the size and connectivity pattern of the matrix equations that it must solve.

- The Map Connections (MC) Procedure is called after the connectivity pattern is determined for the entire IMS. The MC Procedure creates a mapping array that maps the position of GWF Model cells and their connections within the system of equations managed by the IMS. This mapping information is used by the GWF Model as part of the formulate procedures to add terms to the system of equations.

- The Allocate and Read (AR) Procedure performs a number of setup functions. Hydrologic options are determined and memory is allocated for simulation components that were not allocated as part of the DF Procedure. Data that do not vary from stress period to stress period are also read as part of the AR procedure. These data include the following: some boundary information, initial heads (starting heads), and aquifer hydraulic properties. Certain preliminary calculations also are made in the AR procedure to prepare data for further processing. 


\section{B-2 Documentation for the MODFLOW 6 Groundwater Flow Model}

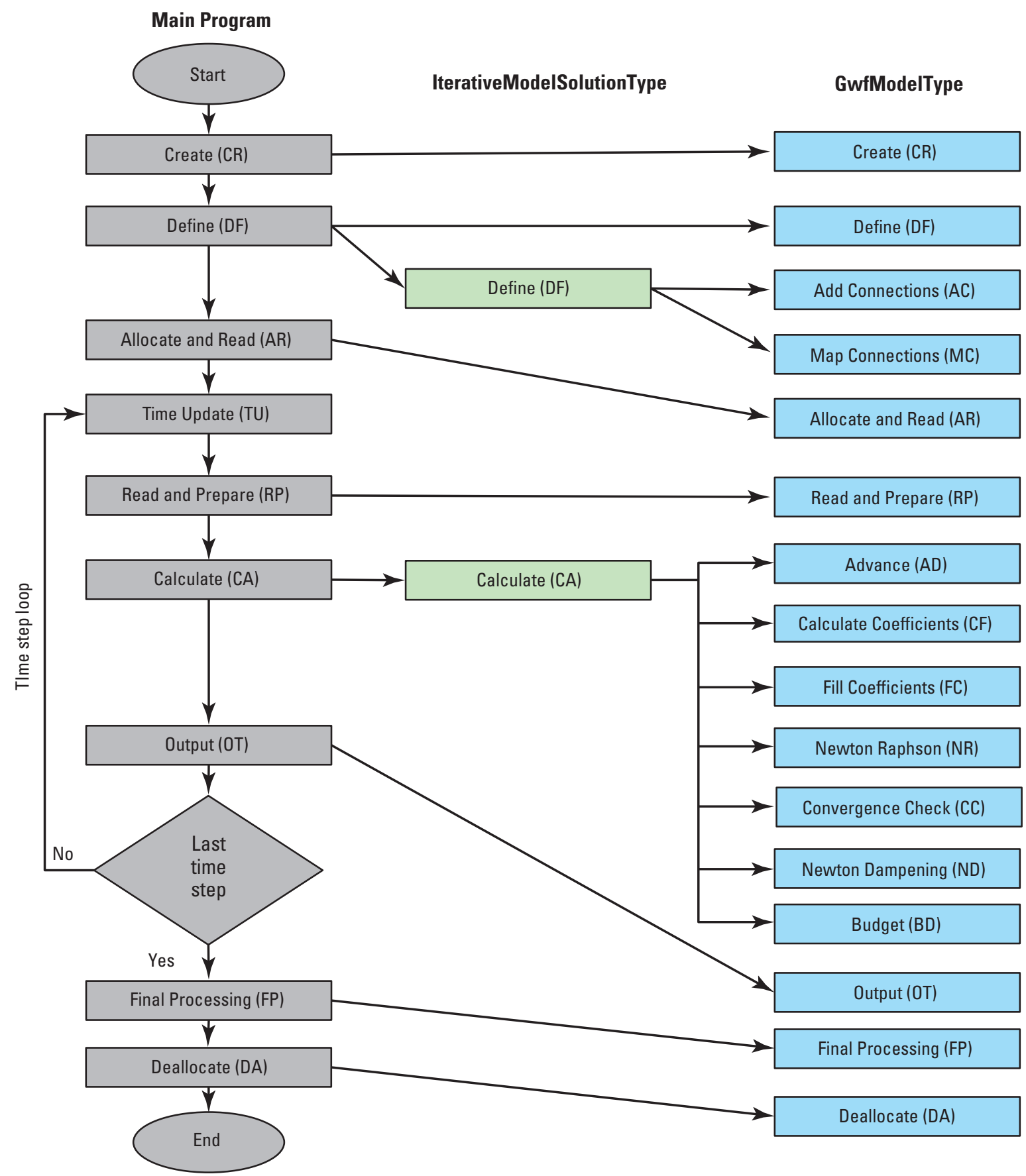

Figure B-1. Schematic diagram showing how primary procedures of the Groundwater Flow (GWF) Model are called from the Main Program and from the Iterative Model Solution. 
- The Read and Prepare (RP) Procedure is called at the start of every time step, but only reads and processes blocks of information if it is the first time step in a stress period. This information includes pumping rates and areal recharge, for example.

- The Advance (AD) Procedure is called to update values for the time step. The AD Procedure initializes the heads, performs time-series substitution, and calls AD Procedures for individual boundary packages. The AD Procedure also performs other processing that must be done at the beginning of each time step.

- The Coefficient Formulate $(\mathrm{CF})$ procedure calculates terms that are needed in subsequent procedures. The CF Procedure also rewets cells, if necessary, and makes dry cells inactive.

- The Fill Coefficients (FC) Procedure adds coefficients to the A coefficient matrix and to the b right-handside vector.

- The Newton Raphson (NR) Procedure adds Newton Raphson terms to the A coefficient matrix and to the b right-hand-side vector. The NR Procedure is invoked only if the Newton Raphson formulation is used for the GWF Model.

- For some advanced boundary packages, it may be necessary to perform a separate convergence check to ensure that flows between the aquifer and the advanced package have converged. Without this check, it is possible that the flow seen by the aquifer has not converged with the corresponding flow seen by the advanced package. This check is performed as part of Convergence Check (CC) Procedure.

- Another Newton Raphson procedure, called the Newton Dampening (ND) procedure, is then called to dampen large and unrealistic head changes. Specifically, if a simulated head for a cell is below the bottom of the model, then the simulated head is adjusted upward toward the bottom of the model.

- The Budget (BD) procedure calculates flow budget terms for the GWF Model and its boundary packages.

- The Final Processing (FP) Procedure writes final model output and performs some final processing tasks for the model.

- The Deallocate (DA) Procedures deallocates memory associated with the GWF Model.

\section{Packages}

Although the procedures are fundamental components of the computer program, many MODFLOW users prefer to think of the program in terms of its capabilities for solving hydrologic problems. For this purpose, the GWF Model is divided into packages, as was done in all previous MODFLOW versions. The various parts of the code that deal with defining the groundwater flow equation are divided into packages that are called hydrologic packages. There are three types of hydrologic packages. The first type is the internal flow package, which contributes to the calculation of flow between adjacent cells or handles storage changes for all the model cells. The second type of package is the Hydrologic/Stress package, which simulates a simplified and individual kind of stress (such as rivers, wells, and recharge). Lastly, the Hydrologic/Advanced Stress Package simulates more advanced stresses. The more advanced stresses typically involve solving some form of a water-budget equation for the stress features, such as a stream, lake, multi-aquifer well, or unsaturated zone.

Table B-1 lists the various packages of GWF that are documented in this publication, the three-character abbreviation used for each package, and the package category. Table B-1 does not show anything about the programming, but shows the capabilities of the program. The Hydrologic/Internal Packages calculate the coefficients of the finite-difference equation for each cell or store information needed to calculate these coefficients. The Discretization (DIS, DISV, DISU) Packages calculate or manage cell surface areas and volumes, 


\section{B-4 Documentation for the MODFLOW 6 Groundwater Flow Model}

and the geometric properties of the connections between cells. The Initial Conditions (IC) Package reads the starting heads for a simulation. The Node-Property Flow (NPF) calculates hydraulic conductance between adjacent cells, manages wetting and drying of cells, and calculates the flow between adjacent cells. The Horizontal Flow Barrier (HFB) Package is a supplementary internal flow package that works with the NPF Package to modify conductances to simulate a barrier between horizontally adjacent nodes. The Ghost Node Correction (GNC) Package is a supplementary internal flow package that works with NPF to improve the accuracy of flow calculations for some grid types. The Storage (STO) Package calculates the change in water volume that occurs over a time step. Only one instance of each of the Hydrologic/Internal Packages listed in Table B-1 can be used for a GWF Model; for example, there can be only one STO Package used for a GWF Model.

Each Hydrologic/Stress Package formulates the coefficients describing a particular external or boundary flow; for example, the River Package calculates the coefficients describing flow between a cell and a surface river. Packages in the Hydrologic/Stress category include: CHD, WEL, RCH, RIV, GHB, DRN, and EVT. These seven packages are described by Harbaugh (2005) and provide core MODFLOW functionality that has been available since the first MODFLOW release. The MAW, SFR, UZF, and MVR Packages are more complicated than the seven core stress packages. For this reason, they are grouped into the Hydrologic/Advanced Stress category. A unique capability of MODFLOW 6 is that multiple Hydrologic/Stress and Hydrologic/Advanced Stress Packages of the same type can be included in a single GWF Model. This new MODFLOW capability was not available in previous MODFLOW versions.

The only packages that do not fit into the hydrologic or categories are the Observation (OBS) and the Output Control (OC) Packages, which manage the printing and saving of GWF Model results to output files. The Output Control Package indicates the time steps for which flows are to be saved. In addition, each hydrologic package includes a keyword option that is set if the flow terms computed by that package are to be saved. Thus, if the appropriate flag in the Evapotranspiration Package input is set, evapotranspiration flow terms will be saved for each time step for which the saving of flows is requested through the OC Package. The OBS Package allows the head in a cell or flows for a cell to be written to a separate output file. Heads and flows can be written for a single point in time or they can be written as a continuous dataset. These OBS and OC Packages are grouped in the Output category shown in table B-1. A GWF Model can contain, at most, one instance of each of these packages.

\section{Primary Routines}

The GWF Model operates by executing a sequence of primary procedures. As part of this process, the GWF Model will call primary procedures for each individual package, if the package has one. The classification of GWF Model primary routines by procedure and by package is illustrated in table B-2. The horizontal rows in table B-2 correspond to primary routines and the vertical columns correspond to packages. An "s" is entered in the block if the procedure is implemented using a Fortran subroutine. An " $\mathrm{m}$ " is entered in the block if the procedure is implemented using a method assigned to the package. An " $\mathrm{i}$ " is entered in the block if the procedure is implemented using an inherited method from the package superclass. An absence of one of these letters indicates that the procedure in question is not required in the indicated package.

The primary routines are named according to a convention that indicates the package and the procedure. The first three characters designate the package. The fourth character is an underscore (_). The last two characters indicate the procedure. For example, in table B-2, a GWF primary routine is indicated for the Well Package and Allocate and Read Procedure. This routine is designated as "wel_ar." Thus, this routine is one that deals with the simulation of specified withdrawal or injection, as through wells, and its particular functions are to allocate the space in computer memory used to store well data and to read well data that are constant during the simulation.

When primary routines become so large that they are difficult to understand, they are broken into smaller secondary routines. If possible, these routines are marked as "private" to indicate that they can only be called 
Table B-1. List of packages for the Groundwater Flow (GWF) Model.

\begin{tabular}{lll}
\hline \hline Package Name & Abbreviation & Package Category \\
\hline Discretization & DIS, DISV, or DISU & Hydrologic/Internal \\
Initial Conditions & IC & Hydrologic/Internal \\
Node Property Flow & NPF & Hydrologic/Internal \\
Horizontal Flow Barrier & HFB & Hydrologic/Internal \\
Ghost Node Correction & GNC & Hydrologic/Internal \\
Storage & STO & Hydrologic/Internal \\
Specified Head & CHD & Hydrologic/Stress \\
Well & WEL & Hydrologic/Stress \\
Recharge & RCH & Hydrologic/Stress \\
General-Head Boundary & GHB & Hydrologic/Stress \\
River & RIV & Hydrologic/Stress \\
Drain & DRN & Hydrologic/Stress \\
Evapotranspiration & EVT & Hydrologic/Stress \\
Stream-Flow Routing & SFR & Hydrologic/Advanced Stress \\
Lake & LAK & Hydrologic/Advanced Stress \\
Multi-Aquifer Well & MAW & Hydrologic/Advanced Stress \\
Unsaturated Zone Flow & UZF & Hydrologic/Advanced Stress \\
Water Mover & MVR & Hydrologic/Advanced Stress \\
Output Control & OC & Output \\
Observation & OBS & Output \\
\hline
\end{tabular}

Table B-2. Groundwater Flow (GWF) Model primary routines classified by procedure and package.

[s is a subroutine (not type-bound); $\mathrm{m}$ is a method (type-bound procedure); $\mathrm{i}$ is an inherited method from the Numerical Package superclass]

\begin{tabular}{|c|c|c|c|c|c|c|c|c|c|c|c|c|c|c|c|c|c|c|c|c|}
\hline & DIS & IC & NPF & HFB & GNC & STO & CHD & WEL & $\mathbf{R C H}$ & GHB & RIV & DRN & EVT & SFR & LAK & MAW & UZF & MVR & OC & OBS \\
\hline Create (CR) & $\mathrm{s}$ & $\mathrm{s}$ & $\mathrm{s}$ & $\mathrm{s}$ & $\mathrm{s}$ & $\mathrm{s}$ & $\mathrm{s}$ & $\mathrm{s}$ & $\mathrm{s}$ & $\mathrm{s}$ & $\mathrm{s}$ & $\mathrm{s}$ & $\mathrm{s}$ & $\mathrm{s}$ & $\mathrm{s}$ & $\mathrm{s}$ & $\mathrm{s}$ & $\mathrm{s}$ & $\mathrm{s}$ & $\mathrm{s}$ \\
\hline Define (DF) & $\mathrm{m}$ & & $\mathrm{m}$ & & $\mathrm{m}$ & & $\mathrm{i}$ & $\mathrm{i}$ & $\mathrm{i}$ & $\mathrm{i}$ & $\mathrm{i}$ & $\mathrm{i}$ & $\mathrm{i}$ & $\mathrm{i}$ & $\mathrm{i}$ & $\mathrm{i}$ & $\mathrm{i}$ & $\mathrm{i}$ & $\mathrm{m}$ & $\mathrm{m}$ \\
\hline $\begin{array}{l}\text { Add Connections } \\
\text { (AC) }\end{array}$ & $\mathrm{m}$ & & $\mathrm{m}$ & & $\mathrm{m}$ & & & & & & & & & & & $\mathrm{m}$ & & & & \\
\hline $\begin{array}{l}\text { Map Connections } \\
\text { (MC) }\end{array}$ & $\mathrm{m}$ & & $\mathrm{m}$ & & $\mathrm{m}$ & & & & & & & & & & & $\mathrm{m}$ & & & & \\
\hline $\begin{array}{l}\text { Allocate and } \\
\text { Read (AR) }\end{array}$ & & $\mathrm{m}$ & $\mathrm{m}$ & $\mathrm{m}$ & & $\mathrm{m}$ & $\mathrm{i}$ & $\mathrm{i}$ & $\mathrm{i}$ & $\mathrm{i}$ & $\mathrm{i}$ & $\mathrm{i}$ & $\mathrm{i}$ & $\mathrm{m}$ & $\mathrm{m}$ & $\mathrm{m}$ & $\mathrm{m}$ & $\mathrm{m}$ & $\mathrm{m}$ & $\mathrm{m}$ \\
\hline $\begin{array}{l}\text { Read and Prepare } \\
\text { (RP) }\end{array}$ & & & & $\mathrm{m}$ & & $\mathrm{m}$ & $\mathrm{m}$ & $\mathrm{i}$ & $\mathrm{m}$ & $\mathrm{i}$ & $\mathrm{i}$ & $\mathrm{i}$ & $\mathrm{m}$ & $\mathrm{m}$ & $\mathrm{m}$ & $\mathrm{m}$ & $\mathrm{m}$ & $\mathrm{m}$ & $\mathrm{m}$ & \\
\hline Advance (AD) & & & $\mathrm{m}$ & & & $\mathrm{m}$ & $\mathrm{m}$ & $\mathrm{i}$ & $\mathrm{i}$ & $\mathrm{i}$ & $\mathrm{i}$ & $\mathrm{i}$ & $\mathrm{i}$ & $\mathrm{m}$ & $\mathrm{m}$ & $\mathrm{m}$ & $\mathrm{m}$ & $\mathrm{m}$ & & $\mathrm{m}$ \\
\hline $\begin{array}{l}\text { Calculate Coeffi- } \\
\text { cients (CF) }\end{array}$ & & & $\mathrm{m}$ & & & & & $\mathrm{m}$ & $\mathrm{m}$ & $\mathrm{m}$ & $\mathrm{m}$ & $\mathrm{m}$ & $\mathrm{m}$ & $\mathrm{m}$ & $\mathrm{m}$ & $\mathrm{m}$ & $\mathrm{m}$ & $\mathrm{m}$ & & \\
\hline $\begin{array}{l}\text { Fill Coefficients } \\
\text { (FC) }\end{array}$ & & & $\mathrm{m}$ & $\mathrm{m}$ & $\mathrm{m}$ & $\mathrm{m}$ & & $\mathrm{i}$ & $\mathrm{m}$ & & & & $\mathrm{m}$ & $\mathrm{m}$ & $\mathrm{m}$ & $\mathrm{m}$ & $\mathrm{m}$ & $\mathrm{m}$ & & \\
\hline $\begin{array}{l}\text { Newton-Raphson } \\
\text { (NR) }\end{array}$ & & & $\mathrm{m}$ & $\mathrm{m}$ & $\mathrm{m}$ & $\mathrm{m}$ & & $\mathrm{m}$ & & & & & & $\mathrm{m}$ & $\mathrm{m}$ & $\mathrm{m}$ & & $\mathrm{m}$ & & \\
\hline \multicolumn{21}{|l|}{$\begin{array}{l}\text { Newton Dampen- } \\
\text { ing (ND) }\end{array}$} \\
\hline Budget (BD) & & & $\mathrm{m}$ & & & $\mathrm{m}$ & $\mathrm{m}$ & $\mathrm{i}$ & $\mathrm{i}$ & $\mathrm{i}$ & $\mathrm{i}$ & $\mathrm{i}$ & $\mathrm{i}$ & $\mathrm{m}$ & $\mathrm{m}$ & $\mathrm{m}$ & $\mathrm{m}$ & $\mathrm{m}$ & & $\mathrm{m}$ \\
\hline Output (OT) & & & $\mathrm{m}$ & & $\mathrm{m}$ & & & & & & & & & $\mathrm{m}$ & $\mathrm{m}$ & $\mathrm{m}$ & & $\mathrm{m}$ & $\mathrm{m}$ & $\mathrm{m}$ \\
\hline $\begin{array}{l}\text { Final Processing } \\
\text { (FP) }\end{array}$ & & & & & & & & & & & & & & & & & & & $\mathrm{m}$ & $\mathrm{m}$ \\
\hline Deallocate (DA) & $\mathrm{m}$ & $\mathrm{m}$ & $\mathrm{m}$ & $\mathrm{m}$ & $\mathrm{m}$ & $\mathrm{m}$ & $\mathrm{m}$ & $\mathrm{m}$ & $\mathrm{m}$ & $\mathrm{m}$ & $\mathrm{m}$ & $\mathrm{m}$ & $\mathrm{m}$ & $\mathrm{m}$ & $\mathrm{m}$ & $\mathrm{m}$ & $\mathrm{m}$ & $\mathrm{m}$ & $\mathrm{m}$ & $\mathrm{m}$ \\
\hline
\end{tabular}




\section{B-6 Documentation for the MODFLOW 6 Groundwater Flow Model}

from a method in the same class. Utility routines are further used to keep the program as easy to understand as possible. Utility routines implement functionality that is required by many packages. When a secondary or utility routine is called, it is logically viewed as being part of the calling routine.

In summary, the GWF Model is broken into primary routines. These primary routines can be grouped according to the procedures indicated in figure B-1. Primary routines can also be grouped by "packages," where a package (for example, the River Package, the Well Package, or the Drain Package) includes those routines required to incorporate a particular hydrologic process into the model. In terms of understanding the operation of the model, both of these ways of grouping routines are useful. The package classification, for example, indicates which routines will be active in a given simulation. Routines are called only if they are part of a package that is used for the GWF model. On the other hand, the procedure classification defines the specific function of a routine in relation to the structure of the computer program. For example, several routines whose function is to allocate space and read data are grouped under the Allocate and Read Procedure; each of these routines allocates the space required for use in a single package. If few options or features are specified by the user, then relatively few packages are involved in the model, and the Allocate and Read Procedure is handled by a relatively small number of routines. As the options specified by the user increase, more packages enter the model, and more routines are called to complete the space allocation task.

\section{Adding and Modifying Packages}

The structure of GWF has been designed in such a way that the packages are as independent as possible. This facilitates making modifications of all types. New packages can be added and the routines of an existing package can be modified without affecting other packages. The method of incorporating stresses through the $H C O F$ and $R H S$ terms allows new stress packages to be used along with the original stress packages. Development of a new package involves creating the primary routines for each of the procedures involved. Through inheritance of a stress boundary package class, the routines will automatically be called in the proper sequence. There is one general constraint that limits what can be done with new packages. Only one internal flow package can be used at a time to formulate the internal flow terms (the NPF Package). For example, a new internal flow package utilizing a different formulation could be added, but using the new package at the same time as the NPF Package would not be possible (or make sense). 
Publishing support provided by the U.S. Geological Survey Science Publishing Network, Reston Publishing Service Center

For information concerning this publication, please contact:

Office of Groundwater

U.S. Geological Survey

Mail Stop 411

12201 Sunrise Valley Drive

Reston, VA 20192

(703) 648-5001

https://water.usgs.gov/ogw/ 


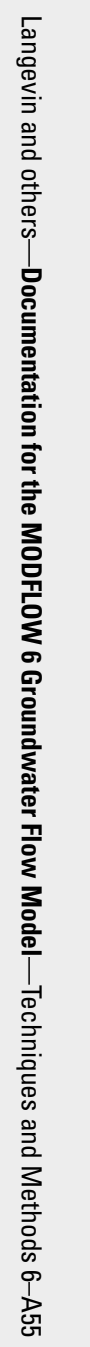

